\title{
Estruturas em larga escala e o Dark Energy Survey
}

\author{
Hugo Orlando Camacho Chavez
}

Orientador: Prof. Dr. Marcos Vinicius Borges Teixeira Lima

Dissertação apresentada ao Instituto de Física da Universidade de São Paulo para a obtenção do título de Mestre em Ciências.

Banca Examinadora:

Prof. Dr. Marcos Vinicius Borges Teixeira Lima (IF-USP)

Prof. Dr. Luis Raul Weber Abramo (IF-USP)

Prof. Dr. Gastão Bierrenbach Lima Neto (IAG-USP)

São Paulo

2014 
FICHA CATALOGRÁFICA

Preparada pelo Serviço de Biblioteca e Informação do Instituto de Física da Universidade de São Paulo

Camacho Chavez, Hugo Orlando

Estruturas em larga escala e o Dark Energy Survey. São Paulo, 2014.

Dissertação (Mestrado) - Universidade de São Paulo. Instituto de Física. Depto. de Física Matemática

Orientador: Prof. Dr. Marcos Vinicius Borges Teixeira Lima

Área de Concentração: Cosmologia

Unitermos: 1. Astronomia; 2. Cosmologia; 3. Estrutura do universo.

USP/IF/SBI-030/2014 


\section{Abstract}

Modern wide-area multi-color deep galaxy redshift surveys provide a powerful tool to probe cosmological models. Yet they bring new practical and theoretical challenges in order to exploit the information contained in their data. This dissertation reviews the theoretical interpretation of clustering of galaxies and shear/convergence weak lensing effects by the large scale structure of the Universe in the context of FLRW cosmological models. This interpretation is general in the sense that the effects of the spatial curvature are properly taken into account, thus holding for FLRW Universes with arbitrary content of matter and dark energy. In this context, we consider two-point statistics both in configuration and harmonic spaces, providing general formulae for the two-point correlation function in real and redshift space. We further include wide angle effects and consider the proper distant observer approximation.

One main characteristic of photometric galaxy surveys is that they will gain in area and depth, in exchange for a poorer determination of radial positions. In this context splitting the data into redshift bins and using the angular correlation function (ACF) $w(\theta)$ and the angular power spectrum (APS) $C_{\ell}$ constitutes a standard approach to extract cosmological information. This dissertation also addresses the problem of constraining cosmological parameters using Bayesian inference techniques from measurements of the ACF and the APS on large scales. Different computational approaches are discussed to accomplish this goal and a detailed model for the ACF at large scales is presented including all relevant effects, namely nonlinear gravitational clustering, bias, redshift-space distortions and photo- $z$ uncertainties.

We present an analysis of the large scale ACF of the CMASS luminous galaxies, a photometric-redshift catalogue based on the Data Release 8 (DR8) of the Sloan Digital Sky Survey-III, showing that the ACF can be efficiently applied to constrain cosmology in future photometric galaxy surveys. We also present a similar analysis on simulated data from the Dark Energy Survey (DES), showing that in the near future such analysis will allow us to constrain cosmological models with even high precision. Finally, we also present preliminary work on the position and shear angular correlations in harmonic space for the Onion simulations. 


\section{Resumo}

Experimentos modernos com observações das posições e redshifts de galáxias em grandes áreas do céu representam uma poderosa ferramenta para a investigação de modelos cosmológicos. Entretanto, estas observações trazem consigo novos desafios práticos e teóricos para a extração da informação contida nos dados. Esta dissertação faz uma revisão da interpretação teórica da aglomeração de galáxias e dos efeitos de lenteamento gravitacional fraco por estruturas em largas escalas no Universo, no contexto de modelos cosmológicos FLRW. Esta interpretação é geral, na medida em que os efeitos da curvatura espacial são apropriadamente considerados, sendo portanto verdadeiros para Universos FLRW com conteúdos artibrários de matéria e energia escura. Neste contexto, consideramos a estatística de dois pontos no espaço de configurações e no espaço harmônico, obtendo fórmulas gerais para a função de correlação de dois pontos no espaço real e no espaço de redshifts. Incluímos ainda efeitos de grandes ângulos e consideramos a aproximação de observador distante de forma apropriada.

Uma característica importante de levantamentos fotométricos de galaxias é a de que eles vão ganhar em área e profundidade, em troca de uma pior determinação das posições radiais. Neste contexto, uma técnica padrão para extração de informação cosmológica dos dados consiste em dividir as galáxias em bins de redshift, de forma a assim usar a função de correlação angular (ACF) $w(\theta)$ e o espectro de potências angular (APS) $C_{\ell}$. Nesta dissertação também tratamos o problema de vincular parâmetros cosmológicos usando técnicas de inferência estatística Bayesiana a partir das medidas da ACF e do APS em grandes escalas. Diferentes técnicas computacionais são discutidas e um modelo detalhado para a ACF em grandes escalas é apresentado, incluindo todos os efeitos relevantes, como não-linearidades gravitacionais, o bias, distorsões no espaço de redshift, e incertezas nas estimativas de redshifts (photo- $z \mathrm{~s}$ ).

Apresentamos uma análise da ACF em grandes escalas para galáxias do CMASS, um catálogo de redshifts fotométricos baseado no Data Release 8 do Sloan Digital Sky SurveyIII, mostrando que a ACF pode ser eficientemente aplicada para vincular cosmologia em levantamentos fotométricos do futuro. Também apresentamos uma análise similar em dados simulados do Dark Energy Survey, mostrando que no futuro próximo tal análise nos permitirá vincular modelos cosmológicos com precisão ainda maior. Finalmente, apresentamos um trabalho preliminar sobre correlações angulares de posição e shear no espaço harmônico para as simulações Onion. 


\section{Acknowledgments}

First and foremost I would like to thank my advisor Marcos Lima, for all his help, support, guidance and constant encouragement without which this truly would not have been possible. I would also like to thank everyone in the Cosmology group at Departamento de Física Matemática da Universidade de São Paulo (DFMA/IF/USP), for providing the best work environment and for the valuable discussions on the seminars and classes, in particular Michel Aguena for his constant and enjoyable collaboration and friendship and for useful comments and discussions during the preparation of this work, and also Lucas Secco, Leandro Beraldo, Henrique Xavier, Ricardo Landim, Leonardo Duarte, Carolina Queiroz, André Alencar, Arthur Loureiro, Leila Graef, Riis Bachega and Lucas Olivari. I profited profusely from our countless discussions.

I would like to thank all the DES-Brazil team for useful discussions during the preparation of this work. I also like to express my appreciation to the co-referees of my thesis, in particular to Raul Abramo, for his valuable comments and discussions.

I am very grateful to Leonardo Castañeda for introducing me to Cosmology and for his valuable friendship and to all my friends of the Gravitation and Cosmology group at Universidad Nacional de Colombia, in particular to my old friend Sergio Rodriguez for his constant encouragement and valuable friendship through all this years.

I would like to thank to all my family for their constant support and encouragement, without them none of this would be possible, and to my friends Oscar Lopez (RIP), Diego Fabra, Yuber Pérez, Antonio Sanchez, Faiber Alonso, Cristian Duitama, Leonardo Gil, Jonathan Mancilla, Ramiro Beltran, Andrés Manrique, Maria Fernanda Gonzalez and all those outside of this list that would otherwise be very long. I am very grateful for all your teachings.

This work has made use of the computing facilities of the Laboratory of Astroinformatics (IAG/USP, NAT/Unicsul), whose purchase was made possible by the Brazilian agency FAPESP (grant 2009/54006-4) and the INCT-A and the computing facilities of DFMA/IF/USP. Also, during this work I have been supported by Brazilian agencies CAPES and CNPq. 


\section{Contents}

1 Introduction $\quad 1$

1.1 Standard model of cosmology . . . . . . . . . . . . . . . . . . . 3

1.2 Cosmological perturbation theory . . . . . . . . . . . . . 8

1.2.1 Linear growth of structures . . . . . . . . . . . . . . 9

1.2.2 Nonlinear evolution . . . . . . . . . . . . . . . . . . . . 11

1.3 The Dark Energy Survey . . . . . . . . . . . . . . . . . . . . . . . . 18

1.4 LSS cosmological observables . . . . . . . . . . . . . . . . . 18

1.4.1 Three and two-dimensional galaxy clustering . . . . . . . . . . . . 18

1.4.2 Weak gravitational lensing . . . . . . . . . . . . . 20

2 Two-point statistics in the Universe 25

2.1 Configuration space . . . . . . . . . . . . . . . . . . 27

2.1.1 Correlation function and power spectrum . . . . . . . . . . . . 27

2.1.2 Estimation techniques in configuration space . . . . . . . . . . . 31

2.1.3 Comparison of different estimators . . . . . . . . . . . . . . . 37

2.2 Harmonic space . . . . . . . . . . . . . . . . . . . . . . . . 37

2.2.1 Angular power spectrum . . . . . . . . . . . . . 37

2.2.2 Simple estimator for the angular power spectra . . . . . . . . . . 40

3 Statistical inference $\quad 43$

3.1 Bayes' Theorem . . . . . . . . . . . . . . . . . . . . . 44

3.2 Bayesian parameter inference . . . . . . . . . . . . . . . . 46

3.3 MCMC techniques for model parameter Bayesian inference . . . . . . . . . 47

3.3.1 Metropolis-Hastings sampling . . . . . . . . . . . . . . . . . 48

3.3.2 Affine-invariant ensemble MCMC . . . . . . . . . . . . . . . . 48

4 Results 53

4.1 Two-point statistics in configuration space . . . . . . . . . . . 54

4.1.1 Galaxy two-point correlation function in FLRW Universes . . . . . 55

4.1.2 Angular two-point correlation function of galaxies . . . . . . . . . . 64

4.1.3 Computing the multipoles of the two-point correlation function . . 71

4.1.4 SDSS-III DR8 photometric luminous galaxies ACF . . . . . . . . . 75

4.1.5 BCC-Aardvark-v1.0 red galaxies ACF . . . . . . . . . . . . . 87

4.2 Two-point statistics in harmonic space . . . . . . . . . . . . 96

4.2.1 The onion simulation maps . . . . . . . . . . . . . . . . . 97

4.2.2 Angular power spectra measurements and modeling . . . . . . . . . 97

4.2.3 Simple cosmological analysis of APSs from Onion simulations . . . 98 
$\begin{array}{ll}\text { Appendices } & 109\end{array}$

$\begin{array}{ll}\text { A Cosmological perturbations } & 109\end{array}$

A.1 Background Geometry . . . . . . . . . . . . . . . . . . 109

A.2 Perturbation theory and the gauge problem . . . . . . . . . . . 110

A.2.1 Taylor expansion of a tensor field . . . . . . . . . . . . . . . 111

A.2.2 Gauge transformations and gauge invariance . . . . . . . . . . . 112

A.3 First order perturbations . . . . . . . . . . . . . . . . . . . . . 114

A.3.1 Scalar-vector-tensor decomposition . . . . . . . . . . . . . . 114

A.3.2 Metric perturbations . . . . . . . . . . . . . . . 115

A.3.3 First order scalar perturbations . . . . . . . . . . . . . . 116

A.3.4 Matter-energy perturbations . . . . . . . . . . . . . . . . 118

A.3.5 First order Einstein field equations . . . . . . . . . . . . . 119

B Scalar harmonic modes in the Universe 123

B.1 Harmonic decomposition of a scalar field . . . . . . . . . . . . . 130

C Linear redshift space distortions in the Universe 133

C.1 Redshift . . . . . . . . . . . . . . . . . . . . . . . 134

C.2 Redshift-space radial distance and coordinate . . . . . . . . . . . . . . 134

C.3 Redshift-space distorted fluctuations of matter . . . . . . . . . . . 136

C.4 Redshift-space distortion operator . . . . . . . . . . . . . . . . . 139

D Deflection of light rays by LSS in the Universe 141

D.1 Geometric optics approximation . . . . . . . . . . . . . . . . . . 141

D.2 Background null geodesics . . . . . . . . . . . . . . . . . . . 143

D.3 First order perturbed null geodesics and deflection angle . . . . . . . . . 144

$\begin{array}{lr}\text { E FFTLog method } & 149\end{array}$

E.1 Discrete Fourier transform . . . . . . . . . . . . . . . . . . . . . . 149

E.2 Discrete Hankel transform and FFTLog method . . . . . . . . . . . . . . 150

E.3 Mellin transform of Bessel functions of the first kind . . . . . . . . . . . . . 152

$\begin{array}{ll}\text { Bibliography } & 155\end{array}$ 


\section{Chapter 1}

\section{Introduction}

One of the main challenges in modern cosmology is the study of the large-scale structure of the observable Universe, its relation to astrophysical phenomena such as galaxy formation on the one hand and to the Universe evolution on large scales on the other hand. In particular, the latter may help shed light on yet unknown fundamental physics, which seems necessary to explain recent observations.

In recent years cosmology entered what can be called a "golden age", due to two fundamental reasons: (a) our knowledge about the Universe appears to be consolidating along with all observational data that seem to converge consistently into a standard cosmological model (a concordance model) and (b) on top of this consolidation, cosmology faces many theoretical and practical challenges. The theoretical challenges are mostly related to the physical nature of the constituents of the concordance model, as for example, the nature of dark matter, of dark energy or cosmic acceleration, and of inflation. The observational challenges are mainly related with the variety of observational probes proposed, their proper interpretation and the necessity of dealing with large amounts of data that are expected from current and upcoming observations.

Among these challenges, the mystery and fundamental implications of cosmic acceleration have inspired numerous ambitious observational efforts, with the goal of measuring the Universe expansion history and possibly reveal its origin. An important step in this process was the production of the report of the Dark Energy Task Force (DETF; [1]), a fundamental effort in defining the problem, categorizing the observational approaches and providing a quantitative framework to compare their capabilities. The DETF focused on four cosmological observables: Type-Ia supernovae (SNIa), clusters of galaxies, clustering of galaxies and baryon acoustic oscillations (BAO) and weak gravitational lensing. The last three probes are fundamentally related with the large scale structure of the Universe.

In fact, the large scale structure of the Universe (LSS) constitutes a promising probe for most of the theoretical challenges of cosmology, especially inflation and cosmic acceleration. For the first challenge, the reason is that the seed perturbations for cosmic structures are settled by the inflationary period, described by specific inflationary models. Most of these models predict initial perturbations to be nearly scale invariant and to obey nearly Gaussian statistics. However, the extent to which they deviate from perfectly scale invariant Gaussian fluctuations depends on the details of the model. Therefore, by accurately measuring deviations from scale invariance as well as from Gaussianity, one can in principle constrain the physics of inflation. For the second challenge, dark energy controls the expansion history of the Universe, which in turn affects how one observes astrophysical objects such as galaxies cataloged by their angular positions and redshifts. 
The relation between redshift and physical distances depends on the expansion rate and the spatial geometry of Universe. In addition, the expansion rate slows down the gravitational evolution of cosmic structure. Therefore, by measuring the distances and growth of cosmic structures, one can constrain the properties of dark energy.

This dissertation is organized as follows. This first chapter sets the foundations of the work, as the standard cosmological model is reviewed. We do not intend to provide an exhaustively complete revision, but only the most fundamental aspects that will be necessary later. Our treatment is based on the original treatments of textbooks $[2,3,4]$ and the Cargèse lectures of 1998 [5], which we recommend for further discussions. In section 1.2 we consider perturbations around the background expansion following the same textbooks and original developments presented on [6]. In section 1.3 we briefly discuss the Dark Energy Survey. The chapter ends in section 1.4, where two of the four observational probes considered in the DETF are interpreted in the context of FLRW Universes. More precisely, the galaxy number fluctuation field is presented as the basic concept for understanding clustering and the cosmic shear and convergence fields are also presented as analogs for the weak lensing phenomena by LSS in the Universe. Our discussions follow the original works $[7,8]$ and [9] in the galaxy clustering section and the review articles $[10,11]$ as well as $\S 7.1$ of [3] for the weak lensing section, which we recommend for further details. Some further theoretical details and computations are also left for the appendices.

In chapter 2, the two-point statistics of these fields is considered and its relation to the total matter power spectrum today is derived. This relation is the basic tool to properly compare cosmological observations with theoretical models.

In chapter 3, Bayesian statistical inference methods are reviewed as the basic tool to constrain cosmological parameters from observations. In this context the sampling problem is depicted and the widely used Markov Chain Monte Carlo (MCMC) methods are presented as a reliable solution. The recent method of affine-invariant MCMC is also discussed as a powerful alternative for solving efficiently the problems of sampling degenerate probability distribution functions with the possibility of using parallel computational resources.

In chapter 4 , the main results of the present dissertation are presented. Initially a general formula for the two-point correlation function of galaxies in redshift space is presented, accounting for wide angle effects, arbitrary redshifts and spatial curvature. From them, the distant observer approximation is considered. Then, a model for the angular correlation function of galaxies at large scales is presented, accounting for nonlinear gravitational clustering, bias, redshift space distortions and photo- $z$ uncertainties. An analysis of the large-scale angular correlation function is presented for the CMASS luminous galaxies (LGs), a photometric-redshift catalog based on the Data Release 8 (DR8) of the Sloan Digital Sky Survey-III, showing that the ACF can be efficiently applied to constrain cosmology in future photometric galaxy surveys. The results of this work have been published in [12]. Another analysis of the ACF on large scales but for a simulated catalog of the DES collaboration is also presented. We end the chapter with an analysis of simple measurements of the APS from galaxy positions on the Onion Universe Simulation [13]. Measurements of the auto-correlation of the convergence field and the cross correlation of convergence and galaxy positions are also considered but are not used on the cosmological analysis.

Finally, we conclude the present dissertation with a summary and outlook in chapter 5. 


\subsection{Standard model of cosmology}

The Universe is observed to be isotropic about us to a high degree of confidence, once we (a) average over large enough scales, considerably larger than the typical scales of clusters of galaxies, and (b) allow for an observer peculiar velocity relative to the average motion of matter in the Universe. In practice, this velocity is treated as relative to the microwave background radiation $^{1}$. In other words, on cosmologically observable scales, there is no particular direction that can be stated to be the center of the Universe. One ends up with two possibilities: (a) either the Universe is spatially homogeneous, and as an specific observer we are on a typical place as the Universe is isotropic for any typical observer, or (b) the Universe is spatially inhomogeneous, and we are near a distinguished place with respect to which the Universe looks isotropic. The common choice of the modern scientific community is the former one. It is commonly interpreted as a Copernican principle, i.e., the assumption that we are not at a privileged position in the Universe $[14,2]$. The Copernican principle along with the observed isotropy are sufficient conditions for the global spatial homogeneity of the Universe [2].

The modern standard cosmological model then assumes that the Universe is spatially homogeneous and isotropic. Combined, these two assumptions are commonly known as the cosmological principle. Spatial homogeneity imply the existence of a one-parameter family of space-like hypersurfaces $\Sigma_{t}$, foliating the spacetime, in which the Copernican principle is valid and therefore every point is equivalent. On the other hand, spatial isotropy imply the existence of a congruence of time-like worldlines with tangent vector $u^{a}$ defining the four-velocity of the so-called isotropic observers, such that it is impossible to construct a preferred tangent vector perpendicular to $u^{a}{ }^{2}$. Combining both conditions imply that the four-velocity of isotropic observers $u^{a}$ and the homogeneity hypersurfaces $\Sigma_{t}$ should be perpendicular, otherwise the Universe should have a privileged spatial direction violating isotropy. Then, viewed as three-dimensional subspaces, the $\Sigma_{t}$ surfaces are maximally symmetric, and consequently are spaces of constant curvature [14, 2]. The isotropic observers acquire the property that for each instant of proper time, they observe a maximally symmetric 3D space, which is why they are also called fundamental observers.

Therefore, one can define a coordinate time $t$, the cosmic time, as the proper time measured by the fundamental observers, $\mathrm{d} t=\mathrm{d} x^{a} u_{a}$ in terms of which the metric of spacetime can be written as

$$
g_{a b}=u_{a} u_{b}+\widehat{\gamma}_{a b}(t),
$$

where for each value of $t, \widehat{\gamma}_{a b}(t)$ determines the metric of the constant time hypersurfaces $\Sigma_{t}$. Since this hypersurfaces should be of constant curvature for each cosmic time instant, one can choose comoving spatial coordinates $\left(x^{i}\right)$ to separate the time dependence and write the spacetime metric tensor as

$$
\overline{\mathrm{g}}=-\mathrm{d} t \otimes \mathrm{d} t+a^{2}(t) \gamma_{i j} \mathrm{~d} x^{i} \otimes \mathrm{d} x^{j},
$$

where the function $a(t)$, giving the time evolution of the hypersurfaces $\Sigma_{t}$, is the cosmic scale factor, determining how physical spatial scales change with time and relate to the comoving scales. The spatial metric of components $\gamma_{i j}$ in comoving coordinates defines

\footnotetext{
${ }^{1}$ See Appendix $\mathrm{C}$ for a discussion of this point.

${ }^{2}$ Throughout this work the index convention is such that spacetime and spatial indices with respect to a general basis are denoted by $a, b, \cdots=0,1,2,3$ and $\alpha, \beta, \cdots=1,2,3$ respectively, while spacetime and spatial indices in a coordinate basis are $\mu, \nu, \cdots=0,1,2,3$ and $i, j, \cdots=1,2,3$, respectively.
} 
generic 3D spaces of constant curvature. A spacetime metric with the form of (1.2) and the above characteristics is known as a Robertson-Walker metric (RW).

For the purposes of this work it is useful to introduce the conformal time $\eta$ by the relation $a \mathrm{~d} \eta=\mathrm{d} t$, in terms of which the RW metric (1.2) reads

$$
\overline{\mathrm{g}}=a^{2}(\eta)\left[-\mathrm{d} \eta \otimes \mathrm{d} \eta+\gamma_{i j} \mathrm{~d} x^{i} \otimes \mathrm{d} x^{j}\right] .
$$

For the constant time hypersurfaces one can always choose spherical coordinates $x^{i}=$ $(\chi, \theta, \varphi)$, where $\chi$ is a radial coordinate and $(\theta, \varphi)$ are the usual polar and azimuthal angles of spherical coordinates on the unit sphere $\mathbb{S}_{2}$. During this work $\chi$ is chosen to be adimensional for the spatially non-flat cases. The components of the spatial metric for this coordinates choice are given in the (comoving) spatial line element

$$
\mathrm{d} \ell^{2}=\gamma_{i j} \mathrm{~d} x^{i} \mathrm{~d} x^{j}= \begin{cases}-K^{-1}\left[\mathrm{~d} \chi^{2}+\sinh ^{2}(\chi) \mathrm{d} \Omega^{2}\right] & K<0, \\ \mathrm{~d} \chi^{2}+\chi^{2} \mathrm{~d} \Omega^{2} & K=0, \\ K^{-1}\left[\mathrm{~d} \chi^{2}+\sin ^{2}(\chi) \mathrm{d} \Omega^{2}\right] & K>0\end{cases}
$$

where $\chi \in[0, \infty)$ for $K \leq 0, \chi \in[0, \pi]$ for $K>0$ and $\theta \in[0, \pi]$ and $\varphi \in[0,2 \pi)$ for all cases in order to cover the spacetime. This choice of coordinates is particularly useful for our purposes because it leaves all the spatial coordinates with the same dimensionality. Note that here $K$ can be interpreted as determining a radius of curvature of constant time hypersurfaces. 3D spaces of constant curvature can be constructed by embedding a 3D hyperboloid, plane and sphere on a 4D flat space for the $K<0, K=0$ and $K>0$ cases, respectively. The radius of each one of these hypersurfaces defined as $|K|^{-1 / 2}$ imply the embedded metric to be given by (1.4) (see e.g., [2]).

One can also choose a fully dimensional radial coordinate (a more frequent choice in the literature) by defining the radial comoving distance,

$$
r= \begin{cases}|K|^{-1 / 2} \chi & K \neq 0 \\ \chi & K=0\end{cases}
$$

Thus for coordinates $x^{i}=(r, \theta, \varphi)$ the comoving spatial line element reads:

$$
\mathrm{d} \ell^{2}=\gamma_{i j} \mathrm{~d} x^{i} \mathrm{~d} x^{j}=\mathrm{d} r^{2}+f_{K}^{2}(r) \mathrm{d} \Omega^{2}
$$

where the function $f_{K}$ depends on the sign of the curvature as

$$
f_{K}(r)= \begin{cases}(-K)^{-1 / 2} \sinh (\sqrt{-K} r) & K<0 \\ r & K=0 \\ K^{-1 / 2} \sin (\sqrt{K} r) & K<0 .\end{cases}
$$

There are other common choices for the spatial coordinates, as e.g. taking the function $f_{K}(r)$ itself as the radial coordinate. Setting $x^{i}=(R, \theta, \varphi)$ with $R:=f_{K}(r)$ the spatial metric reads

$$
\gamma_{i j} \mathrm{~d} x^{i} \mathrm{~d} x^{j}=\frac{\mathrm{d} R^{2}}{1-K R^{2}}+R^{2} \mathrm{~d} \Omega^{2}
$$

When the forms (1.4) and (1.6) are considered on the full RW metric (1.3), for each value of $\eta$ the curvature of spatial sections $\Sigma_{t}$ is given by $K a^{-2}(\eta)$, so that it is possible to 
rescale the scale factor in order to make $K$ to have only three discrete values $-1,0,+1$. In that case, choosing a radial coordinate adimensional/dimensional is equivalent to choosing the scale factor dimensional/adimensional. This kind of choice has the disadvantage of inhibiting the choice of an arbitrary value for the scale factor today, say $a_{0}=1$, which proves to be very useful for cosmological analyses. In fact, Universe models based on the RW metric have as degrees of freedom the function $a(\eta)$, determining the evolution in time of spatial scales, and the constant $K$, determining the curvature of spatial sections of spacetime. When the rescaling of $a(\eta)$ is done, the degree of freedom in $K$ can be thought to be translated to the value of $a_{0}$ plus the sign of $K$.

The gravitational effects of spatial curvature can be characterized by comparing the curvature radius $|K|^{-1 / 2}$ and the radial comoving scales considered $r$. From equations (1.4) or (1.7) one can see that the spatial metric $\gamma$ for the $K \neq 0$ cases reduces to the flat case when $r|K|^{1 / 2}=\chi \rightarrow 0$, and according to the principle of equivalence, this should be independent of the coordinates used. Therefore, when $r|K|^{1 / 2}=\chi \gg 1$ the effects of curvature should be important, in contrast to situations in which $r|K|^{-1 / 2}=\chi \ll 1$ where they should become negligible.

The fundamental observers move on lines defined by constant comoving coordinates, i.e., $x^{i}=$ const., so their four-velocity components are $u^{\mu}=\mathrm{d} x^{\mu} / \mathrm{d} t=\delta_{0}^{\mu}$ on coordinates $x^{\mu}=\left(t, x^{i}\right)$ and consequently $u^{\nu}=a^{-1} \delta_{0}^{\nu}$ on coordinates $x^{\nu}=\left(\eta, x^{i}\right)$.

In order to specify a cosmological model, besides the spacetime geometry, one needs a suitable matter/energy content and a gravitational theory or a specification of the interaction of the geometry and the matter/energy content [5]. Modern Cosmology assumes the former through Einstein's relativistic gravitational field equations (EFE) given by

$$
G_{a b}:=R_{a b}-\frac{1}{2} R g_{a b}=\kappa T_{a b}-\Lambda g_{a b}
$$

where $G_{a b}$ and $R_{a b}$ are the components (on a general basis) of the Einstein and Ricci tensor of the spacetime, respectively, $R$ is the Ricci (or curvature) scalar, $\kappa:=8 \pi G_{N}{ }^{3}, T_{a b}$ are the components of the energy-momentum tensor and $\Lambda$ is the cosmological constant, an spacetime constant in the sense that its covariant derivative is null, i.e. $\nabla_{a} \Lambda=0$. The EFE also guarantee the local conservation of energy and momentum, as the twicecontracted Bianchi identities, $\nabla_{a} G^{a b}=0$, imply $\nabla_{a} T^{a b}=0$ [14].

Any cosmological model with a RW geometry and some suitably specified matter/energy content determining the dynamical evolution according to General Relativity via the EFE (1.9) is called a Friedmann-Lemaître-Robertson-Walker model (FLRW). In this work only FLRW cosmological models are considered. On any FLRW model, as a consequence of the cosmological principle, the only non-zero energy/momentum variables are the energy density $\rho$ and the isotropic pressure $p$. It is important to note that there are no vector nor tensor non-zero energy-momentum degrees of freedom. Furthermore, this scalar fields are all functions of time alone, because of assumptions of homogeneity and isotropy. Thus, fundamental observers on FLRW models measure an energy-momentum tensor, irrespective of the chosen time coordinate, cosmic or conformal, of the form

$$
T_{\mu}^{\nu}=T_{\mu \sigma} g^{\sigma \nu}=\left[\begin{array}{cccc}
-\rho & 0 & 0 & 0 \\
0 & p & 0 & 0 \\
0 & 0 & p & 0 \\
0 & 0 & 0 & p
\end{array}\right] .
$$

\footnotetext{
${ }^{3}$ Throughout this work natural units are assumed $c=\hbar=k_{\mathrm{B}}=1$. Then the gravitational constant in EFE reads $\kappa=8 \pi G_{\mathrm{N}}$, where $G_{\mathrm{N}}$ is the Newton's gravitational constant.
} 
In other words, FLRW Universe models are made up of energy-matter contents that give rise to an effective perfect fluid energy-momentum tensor. From the 10 components of $T_{a b}$ only the two scalar ones are non-zero.

The equations governing the dynamics of FLRW Universe models can be obtained considering the EFE (1.9), for the RW geometry (1.3) with the energy-momentum tensor given by (1.10). In terms of the conformal time $\eta$ the EFE equations are ${ }^{4}$

$$
\begin{aligned}
\left(\frac{a^{\prime}}{a}\right)^{2}+K & =\frac{\kappa}{3} a^{2} \rho+\frac{a^{2} \Lambda}{3} \\
2\left(\frac{a^{\prime \prime}}{a}\right)+\left(\frac{a^{\prime}}{a}\right)^{2}+K & =-\kappa a^{2} p+a^{2} \Lambda
\end{aligned}
$$

and in terms of the cosmic time are

$$
\begin{aligned}
\left(\frac{\dot{a}}{a}\right)^{2}+\frac{K}{a^{2}} & =\frac{\kappa}{3} \rho+\frac{\Lambda}{3}, \\
2 \frac{\ddot{a}}{a}+\left(\frac{\dot{a}}{a}\right)^{2}+\frac{K}{a^{2}} & =-\kappa p+\Lambda .
\end{aligned}
$$

The local conservation of energy/momentum is contained on systems (1.11) and (1.12) because of the Bianchi identities and can be expressed by

$$
\rho^{\prime}+3 \frac{a^{\prime}}{a}(\rho+p)=0 \quad \text { and } \quad \dot{\rho}+3 \frac{\dot{a}}{a}(\rho+p)=0,
$$

in conformal and cosmic time respectively.

The systems of equations (1.11) or (1.12) are known as the Friedmann equations and relate the rate of expansion/contraction of the Universe with its matter/energy content and its spatial curvature. On the other hand equations (1.13) describe the energy conservation on the Universe.

When $a \neq 0(1.12 b)$ is easily readable from (1.12b) and the last equation in (1.13). Therefore, just the Friedmann equation (1.12b) and the conservation equation (1.13) need to be satisfied. It is very useful and also a common practice in the literature to write the Friedmann equation in adimensional form. Therefore, dimensionless density parameters are introduced as

$$
\Omega_{i}(\eta)=\frac{\rho_{i}(\eta)}{\rho_{\text {crit }}}, \quad \Omega_{\Lambda}=\frac{\Lambda}{3 H^{2}},
$$

where $\rho_{\text {crit }}:=3 H^{2} / \kappa$ is the critical density, corresponding to the evolution that the energy density should have in the exact case of a spatially flat Universe, with $H:=\dot{a} / a$ the Hubble parameter. A "density" parameter for curvature can also be introduced as $\Omega_{K}(\eta)=-K / a^{2} H^{2}$ in terms of which the Friedmann equation (1.12b) becomes

$$
\Omega+\Omega_{\Lambda}+\Omega_{K}=1
$$

The density parameter $\Omega$ here represents the contribution to the energy density of all matter fields present, baryons, cold dark matter (CDM), neutrinos, etc., but not the cosmological constant. It is also useful to separate the radiation and matter contributions,

\footnotetext{
${ }^{4}$ Throughout this work the prime symbol ' denotes derivative with respect to conformal time $\eta$ and the dot ' with respect to cosmic time $t$.
} 
$\Omega=\Omega_{\mathrm{m}}+\Omega_{\mathrm{r}}$, because of their different evolutions. We can further split matter naively into CDM and baryons as $\Omega_{\mathrm{m}}=\Omega_{\mathrm{c}}+\Omega_{\mathrm{b}}$. The conservation equation (1.13) is easily solved for perfect fluids with equation of state (EOS) $w=p / \rho=$ constant. For species $i$, one finds ${ }^{5}$

$$
\rho_{i}(a)=\rho_{i 0}\left(\frac{a_{0}}{a}\right)^{3\left(1+w_{i}\right)}=\Omega_{i}(a) \rho_{\text {crit }}(a)
$$

Taking into account that for pressureless matter $w=0$ and for radiation $w=1 / 3$ the Friedmann equation can be written in adimensional form as

$$
\begin{aligned}
& E^{2}(a):=\frac{H^{2}(a)}{H_{0}^{2}}=\frac{\Omega_{\mathrm{m} 0}}{a^{3}}+\frac{\Omega_{\mathrm{r} 0}}{a^{4}}+\frac{\Omega_{K}}{a^{2}}+\Omega_{\mathrm{DE} 0} \frac{\rho_{\mathrm{DE}}(a)}{\rho_{\mathrm{DE} 0}} \\
& E^{2}(z):=\frac{H^{2}(z)}{H_{0}^{2}}=\Omega_{\mathrm{m} 0}(1+z)^{3}+\Omega_{\mathrm{r} 0}(1+z)^{4}+\Omega_{K}(1+z)^{2}+\Omega_{\mathrm{DE} 0} \frac{\rho_{\mathrm{DE}}(z)}{\rho_{\mathrm{DE} 0}},
\end{aligned}
$$

where we introduced the time-dependent function $E$ as the Hubble parameter normalized by its value today and on the second line $(1+z):=a^{-1}$ defines the cosmological redshift $z$ (see $\S$ C.1) and a general model for Dark Energy (DE) is considered. When it is assumed only as a cosmological constant, we replace $\Omega_{\mathrm{DE}}$ by $\Omega_{\Lambda}$, and because it is constant $\rho_{\mathrm{DE}}(z)=$ $\rho_{\mathrm{DE} 0}$; otherwise it is described by the energy density $\rho_{\mathrm{DE}}$ and may change with time. It is also a common practice in the literature to describe the Hubble parameter evolution with the dimensionless variable $h:=H_{0} / 100 \mathrm{Km} \mathrm{s}^{-1} \mathrm{Mpc}^{-1}$.

The comoving distance at a given redshift $z$, is given by the distance-redshift relation,

$$
r(z):=\int_{0}^{z} \frac{\mathrm{d} z^{\prime}}{H\left(z^{\prime}\right)}=\frac{1}{H_{0}} \int_{0}^{z} \frac{\mathrm{d} z^{\prime}}{E\left(z^{\prime}\right)}
$$

where $E(z)$ describes the expansion history of the Universe according to the Friedmann equation (1.17). On the past light-cone, $r(z)$ is related to the adimensional radial comoving coordinate according to

$$
\chi(z)= \begin{cases}|K|^{1 / 2} r(z), & K \neq 0 \\ r(z), & K=0 .\end{cases}
$$

Note that, according to this relation, on the light-cone surface the variable $z$ and the coordinates $t$ and $\chi$ are equivalent. That is because of the physical interpretation of $r(z)$ as the comoving distance travelled by a photon propagating in a radial null geodesic from a point of radial coordinate $\chi$ to the observer (assumed at $\chi=0$ without loss of generality).

During the last decade of the last century, a major discovery was made in Cosmology: the scientific community reached the conclusion that dark and ordinary matter were insufficient to describe accurately a variety of cosmological observations within the framework of the standard cosmological model just depicted above, i.e., a RW metric describing the spacetime and the validity of GR on cosmological scales. The relation between luminosity and distance of type Ia supernovae revealed that in the context of the standard model about $73 \%$ of the total energy density in the Universe comes from an extra component, which causes the Universe not only to expand, but to do it in an accelerated way (i.e., $\ddot{a}>0$ ), $[15,16]$. The more recent results for the observational evidence of the energy/matter content of the Universe come from measurements of the temperature

\footnotetext{
${ }^{5}$ Throghout this work quantities indexed by a 0 are defined to be evaluated today.
} 
fluctuations in the cosmic microwave background (CMB) radiation as determined by the Planck mission [17]. These results have been shown to be consistent with the so-called $\Lambda \mathrm{CDM}$ concordance model of cosmology, consisting of a nearly spatially flat Universe, determined to an accuracy of better than a percent, dominated by two unknown components, the dark matter and dark energy, with $26.8 \%$ and $68.3 \%$ of the total energy content in the Universe respectively, and with only $4.9 \%$ of ordinary matter, i.e. baryons. See [17] for a more detailed discussion.

The nature of dark matter and dark energy or the accelerated expansion of the Universe constitute two of the most important open problems in Physics today. The most basic model of dark energy (DE) describes it as a cosmological constant $\Lambda$, for which density and pressure are constant and related by the equation of state parameter $w=p / \rho=-1$. A large number of alternative models has been explored in recent years. They are mostly separated in two groups: (a) models in which DE is, in fact, a gravitational source in the context of GR, and then is modeled as an evolving field, like e.g., quintessence, see e.g., $[18,19,20]$, and (b) models in which the theoretical basis of gravitational phenomena is proposed to be changed, i.e., the equations of GR are modified in order to describe the acceleration as a dynamical (gravitational) effect, e.g. [18, 21].

\subsection{Cosmological perturbation theory}

In GR, we have to solve ten coupled nonlinear partial differential equations for the metric tensor in terms of the gravity sources represented in the energy-momentum tensor: the EFE (1.9). The idea of perturbation theory (PT) is to reformulate the problem as an infinite hierarchy of linear differential equations for deviations of the metric with respect to a known solution of the EFE that defines the background solution of the system considered. In this way, one translates the difficulty from nonlinearity to the infinite number of equations (see e.g. $\S 7.5$ of [22]). The key assumption of the perturbative scheme is, as is a common practice in Physics, that one can truncate the problem at a finite order and still obtain an approximate solution to the original system.

The cosmological principle allows for relatively over-simplified solutions of the EFE (1.9) as we saw in the last section. Physical reality is more complicated, as the distribution of matter is not exactly homogeneous on all scales. On small scales, below some hundred $\mathrm{Mpc}$, one observes a vast variety of structures such as "walls" of matter, filaments, galaxy clusters and galaxies. In addition, given the non-linear nature of the EFE, it is a very difficult task to solve them exactly for more realistic spacetime models. Thus, in order to obtain realistic models to compare with detailed observations, one needs to approximate, aiming to obtain almost-FLRW models representing a Universe that is FLRW-like on large scales, but allowing for generic inhomogeneities on small scales.

The major problem in studying such perturbed models is the gauge problem, related to the arbitrariness on the identification of perturbed and unperturbed (background) physical degrees of freedom, see e.g. $§ 5.2$ of [2] and [23, 24, 25]. Such identification depends on the choice of the coordinate system and thus on the specific spacetime observer. Consequently the dynamical equations written in terms of perturbation variables have as solutions both physical modes and gauge modes, the latter corresponding to variation of gauge choice, i.e. a choice of the coordinate system used, rather than a physical variation of the corresponding background quantity. A way to overcome this problem is to identify proper gauge-invariant modes describing the physical degrees of freedom on the perturbative quantities, i.e. the actual physical observable quantities. A brief review 
of the way to study general perturbations within GR applied to FLRW Universe models is presented in Appendix A.

The fluctuations (perturbations) on the metric and the energy-momentum tensor of a FLRW model can be separated into three different modes: scalar-, vector- and tensor-like, the so-called scalar, vector and tensor decomposition (SVT), which evolve independently in linear theory [2]. In this work we will concentrate on scalar modes, since they connect the metric perturbations to density, pressure and velocity (see $\S$ A.3). Vector-like perturbations are damped by the cosmic expansion and tensor modes are related to the propagation of gravitational waves. Specifically the work will focus mostly on linear scalar perturbations, although when comparing theory to observations, we must also consider non-linear effects which propagate into linear scales.

Linear (first order) scalar perturbations can be generally described by four functions for the metric and four for the energy-momentum tensor according to equations (A.28) and (A.45) respectively. The energy-momentum perturbations can be identified with the following physical quantities: (a) $\delta(\mathbf{x}, \eta)=\rho(\mathbf{x}, \eta) / \rho(\eta)-1$, the density contrast (fluctuation) at point $\mathbf{x}$ and time $\eta$ relative to the mean value $\rho(\eta) ;(\mathrm{b}) v(\mathbf{x}, \eta)$, the peculiar velocity, i.e. the intrinsic velocity of objects with respect to the comoving coordinates; (c) the isotropic $\delta p$ and (d) anisotropic $\Pi$ pressure fluctuations. The solutions for these variables contain modes that depend on the choice of coordinate system, i.e. to a gauge choice. Since scalar degrees of freedom of gauge transformations are characterized by two scalar fields, it is possible to choose a combination of the eight variables above and obtain six scalar gauge invariant quantities (see e.g. [23, 24] and §A.3.3 and §A.3.4).

Since in this work the interest is on the clustering of matter in the Universe, the problem to consider is the evolution of the pressureless fluid (pure dust) describing the total content of matter in the Universe, CDM plus baryonic, for which the energy-momentum tensor can be chosen as, $T_{a b}=u_{a} u_{b} \rho$, with $u^{a}$ the fluid four-velocity and $\rho$ the energy density. The scalar first order EFE in PT contains all the dynamics of the system. In fact, from the six gauge invariant variables, two should be identically null, the isotropic and anisotropic pressure fluctuations and the EFE can then be reduced to four equations forming a closed system, see appendix A for details.

\subsubsection{Linear growth of structures}

On sufficiently large scales, the gravitational evolution of fluctuations in the total matter in the Universe follows linear perturbation theory. Following the previous discussion, one ends up with four independent linear scalar degrees of freedom: two gravitational potentials, equation (A.38), the density fluctuation and the scalar component of the peculiar velocity, equation (A.47). Their evolution is described by four independent equations derived from the EFE and given by (§A.3)

$$
\begin{aligned}
\delta^{\prime}+\left(\nabla^{2}+3 K\right) v & =0 \\
v^{\prime}+\mathcal{H} v+\Phi & =0 \\
\left(\nabla^{2}+3 K\right) \Phi & =a^{2} \frac{\kappa}{2} \bar{\rho} \delta .
\end{aligned}
$$

This system of equations fully describes the problem for gauge invariant degrees of freedom in the context of GR. In equation (1.20) $\mathcal{H}=a^{\prime} / a$ is the conformal Hubble parameter. Recall that primes denote derivatives with respect to the conformal time, $\delta, v$ and $\Phi$ denote the gauge-invariant density contrast, velocity potential and gravitational potential 
inside the particle horizon and are gauge-invariant up to first order. Actually $v$ denotes the longitudinal (the only scalar one) part of the velocity field, i.e., $v^{i}=D^{i} v$, where $D^{i}$ denotes the covariant derivative with respect to the spatial metric $\gamma_{i j}$.

It is possible to eliminate the variables $v$ and $\Phi$ to obtain an evolution equation for the gauge-invariant fluctuation in matter density $\delta$ :

$$
\delta^{\prime \prime}+\mathcal{H} \delta^{\prime}-\frac{3 H_{0}^{2} \Omega_{\mathrm{m} 0}}{2 a(\eta)} \delta=0 .
$$

Note that, as long as the equations describe pressure-less matter, the background evolution is given by

$$
\bar{\rho}(\eta)=\rho_{\text {crit }} \Omega_{\mathrm{m}}(\eta)=\frac{3 H_{0}^{2}}{\kappa} \Omega_{\mathrm{m}}(\eta)=\frac{3 H_{0}^{2}}{\kappa a^{3}(\eta)} \Omega_{\mathrm{m} 0} .
$$

The equation for $\delta$ is separable in the time and spatial coordinates, so the solutions will be written as

$$
\delta\left(\eta, x^{i}\right)=\delta\left(\eta_{0}, x^{i}\right) \frac{D(\eta)}{D\left(\eta_{0}\right)}=\delta_{0}\left(x^{i}\right) \frac{D(\eta)}{D\left(\eta_{0}\right)},
$$

Obviously we can normalize $D$ to any arbitrary time. In this work, by convenience, the normalization is chosen with respect to the present time ${ }^{6}$. Therefore, the time-dependent part of the solution satisfies the equation

$$
D^{\prime \prime}+\mathcal{H} D^{\prime}-\frac{3 H_{0}^{2} \Omega_{\mathrm{m} 0}}{2 a(\eta)} D=0 .
$$

On equation (1.24) one has the freedom to change the time variable for the cosmic time, the scale factor, or the cosmic redshift depending on what is more convenient. The equations for these variables are then given by

$$
\ddot{D}+2 H \dot{D}-\frac{3 H_{0}^{2} \Omega_{\mathrm{m} 0}}{2 a^{3}(t)} D=0,
$$

for the cosmic time,

$$
\frac{\mathrm{d}^{2} D}{\mathrm{~d} a^{2}}+\left[\frac{3}{a}+\frac{\mathrm{d} \ln E(a)}{\mathrm{d} a}\right] \frac{\mathrm{d} D}{\mathrm{~d} a}-\frac{3 \Omega_{\mathrm{m} 0}}{2 E(a) a^{5}} D=0,
$$

for the scale factor, where again $E(a)$ describes the expansion history according to Friedmann equation (1.17), and

$$
\frac{\mathrm{d}^{2} D}{\mathrm{~d} z^{2}}+\left[\frac{1}{(1+z)}-\frac{\mathrm{d} \ln E(z)}{\mathrm{d} z}\right] \frac{\mathrm{d} D}{\mathrm{~d} z}+\frac{3 \Omega_{\mathrm{m} 0}(1+z)}{2 E(z)} D=0,
$$

for the redshift.

The solutions for $D$ depend on the background evolution via the Hubble parameter $H$. Solutions reduced to quadrature can only be obtained for very specific matter-energy contents and DE models. In general, the problem of finding the time evolution of matter fluctuations must be treated numerically, as is actually done in this work.

Consider now the evolution of the velocity field. Combining equations (A.53) with the solution for the matter fluctuations equation (1.23), one arrives to

$$
v^{\prime}+\mathcal{H} v=-\Phi=-\frac{3 H_{0}^{2} \Omega_{\mathrm{m} 0}}{2 a} G(\eta)\left(\nabla^{2}+3 K\right)^{-1} \delta_{0},
$$

\footnotetext{
${ }^{6}$ Quantities with the subscript ${ }_{0}$ denote evaluation on the present time.
} 
where $G$ denotes the growth factor normalized to its value today, $G(\eta)=D(\eta) / D\left(\eta_{0}\right)$.

The homogeneous solution of equation (1.28) is a decaying mode in time, $v_{\text {hom }} \propto a^{-2}$, and an inhomogeneous solution can be obtained as

$$
v=-\mathcal{H} G f\left(\nabla^{2}+3 K\right)^{-1} \delta_{0}=-a H G f\left(\nabla^{2}+3 K\right)^{-1} \delta_{0}
$$

where the function

$$
f(\eta):=\frac{\mathrm{d} \ln (G)}{\mathrm{d} \ln (a)}=\frac{a}{G} \frac{\mathrm{d} G}{\mathrm{~d} a}=\frac{G^{\prime}}{\mathcal{H} G}=\frac{\dot{G}}{H G} .
$$

One may prove that equation (1.29) is actually a solution of the inhomogeneous equation, by separating variables to see that the spatial-dependent part goes as $\left(\nabla^{2}+3 K\right)^{-1} \delta_{0}$ and the time-dependent part satisfies

$$
v^{\prime}+\mathcal{H} v+\frac{3 H_{0}^{2} \Omega_{\mathrm{m} 0}}{2 a} G(\eta)=0,
$$

so that, by comparing with the equation for the growth factor, equation (1.24), the solution can be written as $v(t)=-G^{\prime}(\eta)=-\mathcal{H} G f$.

\subsubsection{Nonlinear evolution}

On scales much smaller than the horizon and restricting the analysis to a spatially flat background, Newtonian physics can be used to describe the structure evolution [26]. The Newtonian equations for an ideal fluid of zero pressure in comoving coordinates are, see e.g., $[26,27]$,

$$
\begin{aligned}
\dot{\delta}+\frac{1}{a} \nabla \cdot[(1+\delta) \mathbf{v}] & =0 \\
\dot{\mathbf{v}}+H \mathbf{v}+\frac{1}{a} \mathbf{v} \cdot \nabla \mathbf{v} & =-\frac{1}{a} \nabla \Phi,
\end{aligned}
$$

where $H=\dot{a} / a$ is the Hubble factor and recall dots denote derivatives with respect to the cosmic time $t$. These are the continuity and Euler equations for the fluid, written in terms of the density fluctuation and comoving coordinates. In conjunction with the Poisson equation $(1.20 \mathrm{c})$ in the spatially flat case,

$$
\nabla^{2} \Phi=4 \pi G a^{2} \bar{\rho} \delta
$$

i.e., the standard Poisson equation for the Newtonian gravitational field in comoving coordinates, this system of equations fully specifies the dynamics of the fluid.

Linearizing the equations of motion (EOM), i.e considering only terms linear in $\delta$ and $\mathbf{v}$, the Newtonian EOM (1.32b) and (1.32b) become

$$
\begin{aligned}
\dot{\delta}+\frac{1}{a} \nabla \cdot \mathbf{v} & =0 \\
\dot{\mathbf{v}}+H \mathbf{v} & =-\frac{1}{a} \nabla \Phi .
\end{aligned}
$$

Thus, along with the Poisson equation (1.33), they are equivalent to the GR system in the spatially-flat case $(K=0)$, equations (1.20c). To see this explicitly, just make a change of variables $t \rightarrow \eta$ and introduce the velocity scalar potential $v$. 
In order to describe the full non-linear evolution we must depart from linear perturbation theory just discussed above. Given the difficulty to find exact solutions of the non-linear dynamical equations (1.32)-(1.33), a perturbative approach can be chosen. Our following discussion on the perturbative approach within the framework of spatial flatness and Newtonian description follows closely that of [27] and $\S 2.2$ of [6], to which we refer the reader for more details and discussion.

We begin by introducing the variable $\theta:=\nabla \cdot \mathbf{v}$, the divergence of the peculiar velocity field. This variable is particularly useful because according to the SVT decomposition, as long as in a spatially flat background $v_{i}=\partial_{i} v$, we found that it is nothing but the Laplacian of the scalar peculiar velocity potential, i.e.,

$$
\theta=\partial_{i} v^{i}=\partial_{i} \partial^{i} v=\nabla^{2} v
$$

so that, by combining with the linear solution (1.29), we see that at linear level it has the solution

$$
\theta=-\mathcal{H} G f \delta_{0}=-a H G f \delta_{0},
$$

that is, in the linear regime the spatial evolution of the $\theta$ is given by the overdensity field of total matter today and its temporal evolution is the same of the scalar peculiar velocity potential, equation (1.29).

Thus, in terms of $\theta$, one can take the Fourier transform of the full non-linear continuity equation (1.32b) and obtain its representation in Fourier space as

$$
a \dot{\delta}(\mathbf{k}, t)+\theta(\mathbf{k}, t)=-\int \mathrm{d}^{3} \mathbf{x} e^{i \mathbf{k} \cdot \mathbf{x}} \nabla \cdot(\delta \mathbf{v})(\mathbf{x}, t) .
$$

One can then perform an integration by parts and write down the $\delta(\mathbf{k})$ and $\mathbf{v}(\mathbf{k})$ fields as Fourier integrals to obtain

$$
a \dot{\delta}(\mathbf{k}, t)+\theta(\mathbf{k}, t)=-\int \frac{\mathrm{d}^{3} \mathbf{k}_{1}}{(2 \pi)^{3}} \int \frac{\mathrm{d}^{3} \mathbf{k}_{2}}{(2 \pi)^{3}} i \mathbf{k} \cdot \mathbf{v}\left(\mathbf{k}_{1}, t\right) \delta\left(\mathbf{k}_{2}, t\right) \int \mathrm{d}^{3} \mathbf{x} e^{i \mathbf{x} \cdot\left(\mathbf{k}-\mathbf{k}_{1}-\mathbf{k}_{2}\right)} .
$$

One now assumes the peculiar velocity field $\mathbf{v}$ to be curl-free. This assumption was implicit on the SVT devomposition $v^{i}=D^{i} v$ in the context of relativistic pertubations as long as the transverse vectorial mode was not considered because we only consider scalar perturbations. In the Newtonian context, this assumption can be justified by noting that for a pressure-less ideal fluid, linear vorticity perturbations, that is, the transverse part of the peculiar velocity decay with time as $a^{-1}$ (see the discussion in §2.3-2.4 of [27]). The velocity then has only a divergence (scalar potential) part $v$, which in Fourier representation is expressed as $\mathbf{v}(\mathbf{k}) \propto \mathbf{k}$, so that on the last integral one can write

$$
\mathbf{k} \cdot \mathbf{v}\left(\mathbf{k}_{1}, t\right)=\left[\mathbf{k} \cdot \hat{\mathbf{k}}_{1}\right]\left[\hat{\mathbf{k}}_{1} \cdot \mathbf{v}\left(\mathbf{k}_{1}, t\right)\right] .
$$

Moreover, the $\mathbf{x}$-integral can be computed to give a Dirac delta function multiplied by $(2 \pi)^{3}$ and thus one finally arrives to

$$
a \dot{\delta}(\mathbf{k}, t)+\theta(\mathbf{k}, t)=-\int \frac{\mathrm{d}^{3} \mathbf{k}_{1}}{(2 \pi)^{3}} \int \mathrm{d}^{3} \mathbf{k}_{2} \delta_{\mathrm{D}}\left(\mathbf{k}-\mathbf{k}_{1}-\mathbf{k}_{2}\right) \alpha\left(\mathbf{k}_{1}, \mathbf{k}_{2}\right) \theta\left(\mathbf{k}_{1}, t\right) \delta\left(\mathbf{k}_{2}, t\right),
$$

with

$$
\alpha\left(\mathbf{k}_{1}, \mathbf{k}_{2}\right):=\frac{\left(\mathbf{k}_{1}+\mathbf{k}_{2}\right) \cdot \mathbf{k}_{1}}{k_{1}^{2}}
$$


In an analogous way to the calculation for the continuity equation, the Euler equation (1.32b), can be written in Fourier space, after combining it with the Poisson equation (1.33) as

$$
a \dot{\theta}(\mathbf{k}, t)+\dot{a} \theta(\mathbf{k}, t)+\frac{3 H_{0} \Omega_{\mathrm{m} 0}}{2 a} \delta(\mathbf{k}, t)=-\int \mathrm{d}^{3} \mathbf{x} e^{i \mathbf{k} \cdot \mathbf{x}}\left[\partial_{i}\left(v_{j} \partial_{j}\right) v_{i}\right](\mathbf{x}, t),
$$

so that, integrating by parts and expanding the fields $\delta$ and $\mathbf{v}$ in Fourier modes, the integral on the right-hand side becomes

$$
-\int \frac{\mathrm{d}^{3} \mathbf{k}_{1}}{(2 \pi)^{3}} \int \frac{\mathrm{d}^{3} \mathbf{k}_{2}}{(2 \pi)^{3}} i \mathbf{k} \cdot \mathbf{v}\left(\mathbf{k}_{1}, t\right)\left[i \mathbf{k} \cdot \mathbf{v}\left(\mathbf{k}_{2}, t\right)+v\left(\mathbf{k}_{2}, t\right)\right] \int \mathrm{d}^{3} \mathbf{x} e^{i \mathbf{x} \cdot\left(\mathbf{k}-\mathbf{k}_{1}-\mathbf{k}_{2}\right)} .
$$

Then, neglecting the curl-part of the velocity field, as before, one arrives to

$$
\begin{aligned}
a \dot{\theta}(\mathbf{k}, t)+\dot{a} \theta(\mathbf{k}, t)+\frac{3 H_{0} \Omega_{\mathrm{m} 0}}{2 a} \delta(\mathbf{k}, t)=-\int \frac{\mathrm{d}^{3} \mathbf{k}_{1}}{(2 \pi)^{3}} \int \mathrm{d}^{3} \mathbf{x} \delta_{\mathrm{D}}\left(\mathbf{k}-\mathbf{k}_{1}-\mathbf{k}_{2}\right) \\
\times \beta\left(\mathbf{k}_{1}, \mathbf{k}_{2}\right) \theta\left(\mathbf{k}_{1}, t\right) \theta\left(\mathbf{k}_{2}, t\right)
\end{aligned}
$$

with

$$
\beta\left(\mathbf{k}_{1}, \mathbf{k}_{2}\right):=\frac{\left|\mathbf{k}_{1}+\mathbf{k}_{2}\right|^{2} \mathbf{k}_{1} \cdot \mathbf{k}_{2}}{2 k_{1}^{2} k_{2}^{2}} .
$$

The last expression is obtained with the requirement that the integrand in (1.44) is symmetric in $\mathbf{k}_{1}, \mathbf{k}_{2}$.

The kernels $\alpha$ and $\beta$, equations (1.41) and (1.45), respectively, describe the coupling between different Fourier modes of the fields $\delta$ and $\theta$ arising from the non-linear terms in the fluid equations of motion (1.32b)-(1.33). In this sense, the evolution of both harmonic modes $\delta(\mathbf{k})$ and $\theta(\mathbf{k})$ at a given wave vector is determined by the mode-coupling of both fields at all pairs of wave vectors $\left(\mathbf{k}_{1}, \mathbf{k}_{2}\right)$ and these should have a sum equal to $\mathbf{k}$ (as expressed by the Dirac delta on the equations) which is consistent with the requirement of spatial homogeneity.

The equations (1.32b)-(1.33) can be easily written for the conformal time $\eta$ as

$$
\begin{aligned}
\delta^{\prime}(\mathbf{k}, \eta)+\theta(\mathbf{k}, \eta)= & -\int \frac{\mathrm{d}^{3} \mathbf{k}_{1}}{(2 \pi)^{3}} \int \mathrm{d}^{3} \mathbf{k}_{2} \delta_{\mathrm{D}}\left(\mathbf{k}-\mathbf{k}_{1}-\mathbf{k}_{2}\right) \\
& \times \alpha\left(\mathbf{k}_{1}, \mathbf{k}_{2}\right) \theta\left(\mathbf{k}_{1}, \eta\right) \delta\left(\mathbf{k}_{2}, \eta\right), \\
\theta^{\prime}(\mathbf{k}, \eta)+\mathcal{H} \theta(\mathbf{k}, \eta)+\frac{3}{2} \mathcal{H} \Omega_{\mathrm{m} 0} \delta(\mathbf{k}, \eta)= & -\int \frac{\mathrm{d}^{3} \mathbf{k}_{1}}{(2 \pi)^{3}} \int \mathrm{d}^{3} \mathbf{k}_{2} \delta_{\mathrm{D}}\left(\mathbf{k}-\mathbf{k}_{1}-\mathbf{k}_{2}\right) \\
& \times \beta\left(\mathbf{k}_{1}, \mathbf{k}_{2}\right) \theta\left(\mathbf{k}_{1}, \eta\right) \theta\left(\mathbf{k}_{2}, \eta\right)
\end{aligned}
$$

This pair of equations are the basis of the standard cosmological perturbation theory (PT) which begins by noting that for an Einstein-de Sitter (purely matter dominated) cosmological model (EdS), that is, one with $\Omega_{\mathrm{m} 0}=1$ and $\Omega_{\Lambda}=0$, where the Friedman equation implies $a(\eta) \propto \eta^{2}$ and $\mathcal{H}(\eta)=2 / \eta$ and, moreover, where the growing mode, according to equation (1.24), evolves as the scale factor, $G(\eta)=a$, and consequently $f(\eta)=1$, equations (1.46) can formally be solved with a perturbatibe expansion of the 
form ${ }^{7}$

$$
\begin{aligned}
& \delta(\mathbf{k}, \eta)=\sum_{n=1}^{\infty} a^{n}(\eta) \delta_{n}(\mathbf{k}) \\
& \theta(\mathbf{k}, \eta)=-\mathcal{H} \sum_{n=1}^{\infty} a^{n}(\eta) \theta_{n}(\mathbf{k})
\end{aligned}
$$

Note that these expansions are actually with respect to the linear density fields, as is desired in any perturbative scheme as long as the perturbative terms are given by the $\mathrm{EOM}$ as

$$
\begin{aligned}
& \delta_{n}(\mathbf{k})=\int \frac{\mathrm{d}^{3} \mathbf{q}_{1} \cdots \mathrm{d}^{3} \mathbf{q}_{n}}{(2 \pi)^{3 n-3}} \delta_{\mathrm{D}}\left(\mathbf{k}-\sum_{i=1}^{n} \mathbf{q}_{i}\right) F_{n}\left(\mathbf{q}_{1}, \ldots, \mathbf{q}_{n}\right) \delta_{1}\left(\mathbf{q}_{1}, 0\right) \cdots \delta_{1}\left(\mathbf{q}_{n}, 0\right), \\
& \theta_{n}(\mathbf{k})=\int \frac{\mathrm{d}^{3} \mathbf{q}_{1} \cdots \mathrm{d}^{3} \mathbf{q}_{n}}{(2 \pi)^{3 n-3}} \delta_{\mathrm{D}}\left(\mathbf{k}-\sum_{i=1}^{n} \mathbf{q}_{i}\right) G_{n}\left(\mathbf{q}_{1}, \ldots, \mathbf{q}_{n}\right) \delta_{1}\left(\mathbf{q}_{1}, 0\right) \cdots \delta_{1}\left(\mathbf{q}_{n}, 0\right),
\end{aligned}
$$

where the integration kernels $F_{n}$ and $G_{n}$ can be obtained from the fundamental mode coupling functions of the fields $\delta$ and $\theta, \alpha$ and $\beta$ (equations (1.41) and (1.45) respectively) according to the recursion relations for $n \geq 2[27]$

$$
\begin{aligned}
& F_{n}\left(\mathbf{q}_{1}, \ldots, \mathbf{q}_{n}\right)=\sum_{m=1}^{n-1} \frac{G_{m}\left(\mathbf{q}_{1}, \ldots, \mathbf{q}_{m}\right)}{(2 n+3)(n-1)}[(2 n+1) \alpha\left(\mathbf{k}_{1}, \mathbf{k}_{2}\right) F_{n-m}\left(\mathbf{q}_{m+1}, \ldots, \mathbf{q}_{n}\right) \\
&\left.+2 \beta\left(\mathbf{k}_{1}, \mathbf{k}_{2}\right) G_{n-m}\left(\mathbf{q}_{m+1}, \ldots, \mathbf{q}_{n}\right),\right] \\
& G_{n}\left(\mathbf{q}_{1}, \ldots, \mathbf{q}_{n}\right)=\sum_{m=1}^{n-1} \frac{G_{m}\left(\mathbf{q}_{1}, \ldots, \mathbf{q}_{m}\right)}{(2 n+3)(n-1)}\left[3 \alpha\left(\mathbf{k}_{1}, \mathbf{k}_{2}\right) F_{n-m}\left(\mathbf{q}_{m+1}, \ldots, \mathbf{q}_{n}\right)\right. \\
&\left.+2 n \beta\left(\mathbf{k}_{1}, \mathbf{k}_{2}\right) G_{n-m}\left(\mathbf{q}_{m+1}, \ldots, \mathbf{q}_{n}\right)\right]
\end{aligned}
$$

On this recursion relations $\mathbf{k}_{1}:=\sum_{j=1}^{m} \mathbf{q}_{j}$ and $\mathbf{k}_{2}:=\sum_{j=m+1}^{n} \mathbf{q}_{j}$. These functions represent the coupling between Fourier modes of the fields $\delta$ and $\theta$ describing the non-linearity of its EOM. Note further that at linear order, i.e., for $n=1$, these two kernels should reduce to unity, i.e. $F_{1}=G_{1}=1$.

For the second-order solutions, i.e., $n=2$, one has [27]

$$
\begin{aligned}
& F_{2}\left(\mathbf{q}_{1}, \mathbf{q}_{2}\right)=\frac{5}{7}+\frac{1}{2} \frac{\mathbf{q}_{1} \cdot \mathbf{q}_{2}}{q_{1} q_{2}}\left(\frac{q_{1}}{q_{2}}+\frac{q_{2}}{q_{1}}\right)+\frac{2}{7} \frac{\left(\mathbf{q}_{1} \cdot \mathbf{q}_{2}\right)^{2}}{q_{1}^{2} q_{2}^{2}} \\
& G_{2}\left(\mathbf{q}_{1}, \mathbf{q}_{2}\right)=\frac{3}{7}+\frac{1}{2} \frac{\mathbf{q}_{1} \cdot \mathbf{q}_{2}}{q_{1} q_{2}}\left(\frac{q_{1}}{q_{2}}+\frac{q_{2}}{q_{1}}\right)+\frac{4}{7} \frac{\left(\mathbf{q}_{1} \cdot \mathbf{q}_{2}\right)^{2}}{q_{1}^{2} q_{2}^{2}}
\end{aligned}
$$

The remarkable feature of the perturbative solutions for EdS cosmological models above is the fact that they are separated for the time and the wave-numbers, i.e., they are made of products of terms that depend only on these variables. However, in general the Universe should not be well described always by an EdS solution. For more general

\footnotetext{
${ }^{7}$ We do not provide here a proof of this statement because it is out of the scope of the present work. However, the reader can see e.g. [27] and references therein.
} 
$\Lambda$ CDM-like cosmological models the mentioned property of separability can be approximatively maintained by allowing for the respective solutions for the linear growth factor $G$ and its logarithmic derivative $f$ (see the discussion at $\$ 2.4 .4$ of [27]). In this sense, equations (1.47) can be replaced by

$$
\begin{aligned}
& \delta(\mathbf{k}, \eta)=\sum_{n=1}^{\infty} G^{n}(\eta) \delta_{n}(\mathbf{k}) \\
& \theta(\mathbf{k}, \eta)=-\mathcal{H}(\eta) f(\eta) \sum_{n=1}^{\infty} G^{n}(\eta) \theta_{n}(\mathbf{k})
\end{aligned}
$$

and remain approximately valid for any $\Lambda \mathrm{CDM}$ cosmology mantaining the same solutions for the wave-number dependent perturbative coefficients, equations (1.48).

The most remarkable application of the PT formalism depicted above is on the construction of a perturbative expansion for the power spectrum of the total matter in the Universe. A definition of the power spectrum will be given in Chapter 2 as the twopoint correlation of Fourier modes of the field of matter fluctuations or equivalently the Fourier transform of the two-point correlation function describing the probability of find overdensities separated by a given distance scale in the Universe. Considering two wavenumbers $\mathbf{k}_{1}$ and $\mathbf{k}_{2}$ the power spectrum of matter $P$ at the instant of conformal time $\eta$ is given by the relation $(2 \pi)^{3} \delta_{\mathrm{D}}\left(\mathbf{k}_{1}-\mathbf{k}_{2}\right) P\left(\left|\mathbf{k}_{1}-\mathbf{k}_{2}\right|, \eta\right):=\left\langle\delta\left(\mathbf{k}_{1}, \eta\right) \delta^{*}\left(\mathbf{k}_{2}, \eta\right)\right\rangle$. Note that it depends only on the norm of the difference of the wave-vectors and also the appearance of the Dirac delta function; these are consequences of the assumption of statistical homogeneity and isotropy of the field of matter fluctuations (see Chapter 2). If we introduce the PT perturbative solutions of equations (1.51) into this definition we end with the mentioned expansion for the power spectrum, which clearly should have the form $P(k, \eta)=\sum_{i, j} P_{i j}(k, \eta)$, where the perturbative terms $P_{i j}$ are given by the two-point correlation of the fluctuation on the matter density field at different orders, $i$ and $j$, in PT scheme,

$$
(2 \pi)^{3} \delta_{\mathrm{D}}\left(\mathbf{k}_{1}-\mathbf{k}_{2}\right) P_{i j}\left(\left|\mathbf{k}_{1}-\mathbf{k}_{2}\right|, \eta\right):=\left\langle\delta_{i}\left(\mathbf{k}_{1}, \eta\right) \delta_{j}^{*}\left(\mathbf{k}_{2}, \eta\right)\right\rangle .
$$

Note then that at all orders the separation property of the PT expansions imply that the temporal part can be separated according to $P_{i j}(k, t)=G^{i+j}(t) P_{i j}(k)$.

At linear order the power spectrum is then given simply as the correlation of the linear fluctuations

$$
P_{\mathrm{PT}}^{(0)}=P_{11}(k, t)=G^{2}(t) P_{\operatorname{Lin}}(k),
$$

where $P_{\text {Lin }}(k)$ is the linear power spectrum today, also known as the initial power spectrum. In the context of the modern $\Lambda \mathrm{CDM}$ concordance model of cosmology, such spectrum is parametrized as $P_{\text {Lin }}(k) \propto k^{n_{s}} T^{2}(k)$, where $n_{s}$ is the primordial scalar spectral index directly related to the initial conditions defined by the inflation and $T^{2}(k)$ is the transfer function, which encodes the information of the linear evolution of matter fluctuations through the radiation domination era and the resulting recombination era of decoupling of matter and radiation [2]. Such transfer function should then be tracked by using the full dynamics of the mixture of the different species presented in the Universe at these stages via the out of equilibrium formalism of the Einstein-Boltzmann system, see e.g., chapter 7 of [4]. During the present work we use modern sophisticated numerical codes devoted to evolve these equations, specifically we used the CAMB code [28] (see the discussion at the beginning of chapter 4). 
The next order contribution to the power spectrum from PT expansion is the sum of two terms, each one of which mixes up two linear power spectra, $P_{\mathrm{PT}}^{(1)}=P_{22}+P_{13}$ where $[27]$

$$
\begin{aligned}
P_{22}(k, \eta) & =\frac{1}{4 \pi^{3}} \int \mathrm{d}^{3} \mathbf{q} F_{2}^{2}(\mathbf{k}-\mathbf{q}, \mathbf{q}) P(|\mathbf{k}-\mathbf{q}|, \eta) P(q, \eta) \\
& =\frac{G^{4}(\eta)}{4 \pi^{3}} \int \mathrm{d}^{3} \mathbf{q} F_{2}^{2}(\mathbf{k}-\mathbf{q}, \mathbf{q}) P_{\operatorname{Lin}}(|\mathbf{k}-\mathbf{q}|) P_{\operatorname{Lin}}(q) \\
P_{13}(k, \eta) & =\frac{3}{4 \pi^{3}} P(k, \eta) \int \mathrm{d}^{3} \mathbf{q} F_{3}(\mathbf{k}, \mathbf{q},-\mathbf{q}) P(q, \eta) \\
& =\frac{3 G^{4}(\eta)}{4 \pi^{3}} P_{\operatorname{Lin}}(k) \int \mathrm{d}^{3} \mathbf{q} F_{3}(\mathbf{k}, \mathbf{q},-\mathbf{q}) P_{\operatorname{Lin}}(q) .
\end{aligned}
$$

And in this way one can continue up to any order desired.

The linear power spectrum and the first three perturbation terms as computed in the framework of PT just depicted above are shown in the left panel of figure 1.1 as a function of the wave-number. Solid and dashed lines denote positive and negative contributions. This figure shows the main problem of PT: with the exception of the linear power spectrum, each term has both positive and negative contributions and does not appear a tendency for the different perturbative contributions to decrease in amplitude with increasing order. This left us with the impossibility to predict the sign and amplitude of any term before computing it explicitly, and consequently makes the decision of where to truncate the PT expansion problematic. By this fundamental reason one finds the statement on the literature that PT can be used to describe only the mildly non-linear regime but not the full non-linear regime, see [29, 30] and references therein.
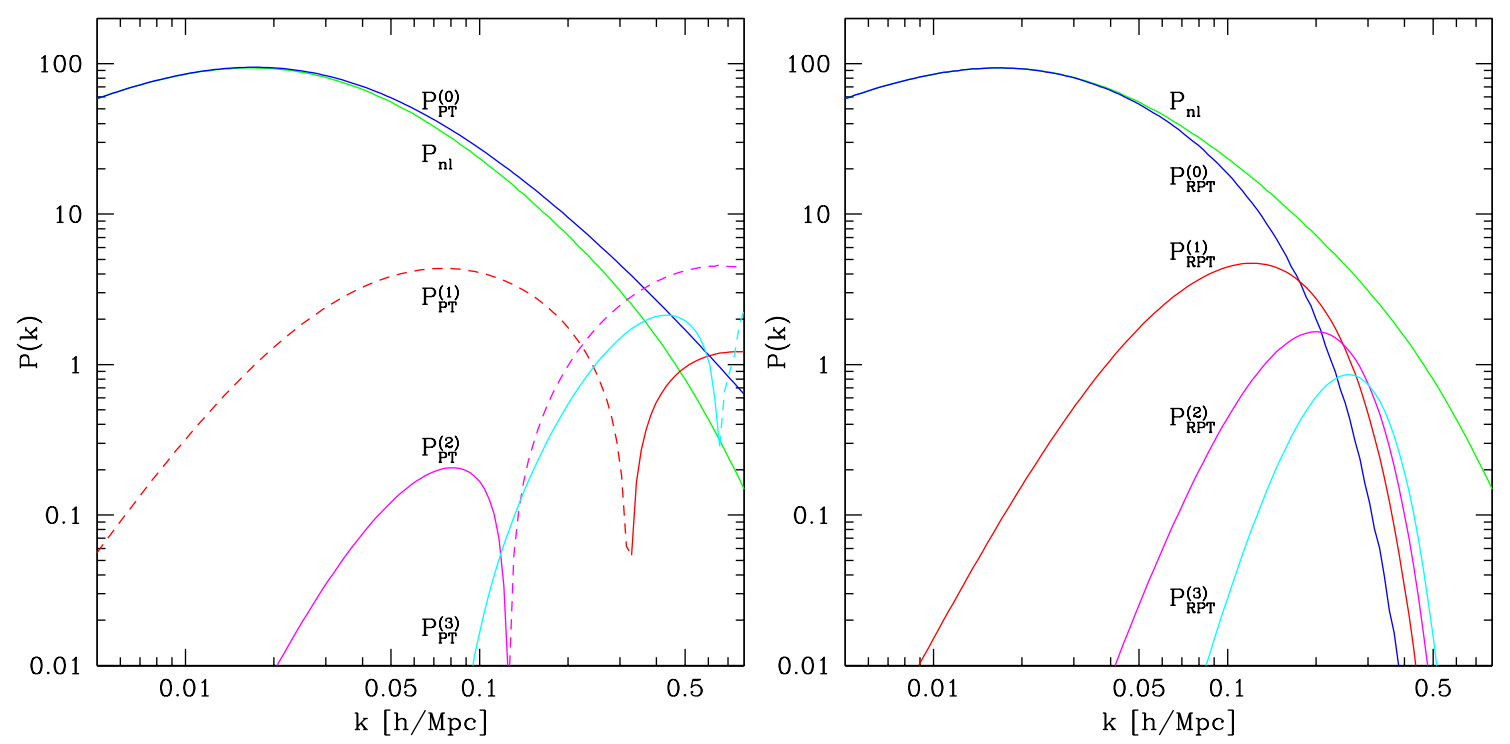

Figure 1.1: Comparison between PT and RPT non-linear power spectrum. Left panel: PT non-linear power spectrum, green line, decomposed according to perturbation theory as the sum of the linear term, blue line, and the first three perturbation terms, red, violet and cyan lines. Solid and dashed lines indicates positive and negative contributions, respectively. Right panel: same non-linear power spectrum decomposed according to RPT. The terms $P_{\mathrm{RPT}}^{(0)}$ and $P_{\mathrm{RPT}}^{(n)}$, for $n>0$ in the figure are equivalent to $G^{2}\left(k, t_{0}\right) P(k, 0)$ and $P_{\mathrm{MC}}^{(n)}\left(k, t_{0}\right)$ from Eq (1.57). Figures from [29] 
Given that the modeling of the two-point statistics of different cosmic fields used in this work requires the proper introduction of the effects of the non-linear evolution of density fluctuations (for the details see $\S 4.1 .2$ and $[8,7]$ ), in this work we made use of the renormalized perturbation theory (RPT) approach $[29,30]$ as an approach to improve PT results. It is out of the scope of the present work to make a detailed review of RPT, which requires a high level of technical developments and tools of field theory. However, we try to mention the main features and basis of this approach. We closely follow the treatment presented on $\S 2.2$ of [6], which can be consulted for detailed discussion.

In an oversimplified way, RPT can be understood as a reorganization of the terms in the PT expansion that remove the problems of this formalism mentioned above. The first idea is that all the terms in the PT expansion for the power spectrum that are proportional to the initial power spectrum, here $P(k, t)$, like $P_{13}$ (see equation (1.54b)) and $P_{15}$ (see e.g., $[27,29]$ ), are grouped together into a common factor $G(k, t)$, the so-called renormalized propagator, which can be interpreted as encoding the loss of information of the initial conditions due to non-linear evolution. At very large scales, the low- $k$ limit, the renormalized propagator should evolve as the growth factor, $G(k, t) \approx G(t)$, having no dependence on the initial conditions. Meanwhile, at small scales, the high- $k$ limit, the behavior of the propagator was computed on [29] to have approximatively the form of a Gaussian with zero mean and dispersion given by a characteristic scale determining where linear theory breakdown. Here we call this scale as $r_{\mathrm{NL}}$ following [12] ${ }^{8}$

$$
r_{N L}^{2}=\frac{1}{3} \int \mathrm{d} k \frac{P_{\operatorname{Lin}}(k)}{k^{2}} .
$$

That is, for small scales [30]

$$
G(k, \eta) \approx G(\eta) \exp \left[-\frac{1}{2} k^{2} r_{\mathrm{NL}}^{2}(G(\eta)-1)^{2}\right],
$$

with $G(\eta)$ the linear growth factor.

The remaining terms, those that are not proportional to $P(k, \eta)$, are organized according to the number $n$ of initial modes coupled and grouped into the mode coupling power spectrum $P_{\mathrm{MC}}^{(n)}(k, t)$. As an example, the lower order term is the one that couples two initial power spectra, i.e., $P_{\mathrm{MC}}^{(2)}(k, t)$. It is given by $P_{22}$ of PT (equation (1.54b)) [30]. In this way, the full non-linear power spectrum in RPT formalism should have the following form

$$
P(k, t)=G^{2}(k, t) P_{\text {Lin }}(k)+\sum_{n=2}^{\infty} P_{\mathrm{MC}}^{(n)}(k, t)
$$

The linear power spectrum and the first three perturbation terms as computed in the framework of RPT just depicted above are shown in the right panel of figure 1.1 as a function of the wave-number. Solid and dashed lines denote positive and negative contributions. This figure shows how the main problems of PT are alleviated in the context of RPT each term has positive contributions and appears dominant over a restricted range of wave-numbers, which shows a tendency to increase in the values of $k$ with increasing perturbative order. This shows the advantage of RPT over standard PT. In principle, it is simpler to decide where to truncate the series of equation (1.57) if a given precision at wave-number $k$ is required.

\footnotetext{
${ }^{8}$ Note that this quantity have different names on different works, [30] originally call it $\sigma_{v}$ and [8] call it $s_{\text {bao }}$
} 


\subsection{The Dark Energy Survey}

The Dark Energy Survey (DES) is a new generation galaxy survey designed to study the late cosmic acceleration of the Universe through the dynamics of the expansion and the growth of structures at large scales on cosmological context. The DES is a collaboration of over a hundred researchers from the USA, UK, Spain, Germany, Chile, Switzerland and Brazil.

The main innovation in the project is the development of a new optical CCD camera of 5000 megapixels and 2.2 degrees of field of view called DECam, which has been placed at the Blanco 4 meters telescope located at Cerro Tololo Inter-American Observatory (CTIO) in Chile and saw its first light in September 2012. For over five years it will use $30 \%$ of the telescope available time to carry out a wide-area survey and reach redshifts $0.2 \leq z \leq 1.3-2$ with a depth of $\sim 24$ in magnitude in SDSS broad bands, $g=24.6$, $r=24.1, i=24.3$ and $z=23.9$ over $5000 \mathrm{deg}^{2}$ in the southern galactic sky.

The DES is expected to detect $\sim 100000$ optical galaxy clusters and to measure shapes, redshifts and positions of $\sim 200$ millions of galaxies of all types. It will obtain cosmological information about the physical nature of dark energy via four different methods (cosmological observables):

1. Count and spatial distribution of galaxy clusters with $0.2 \leq z \leq 1.3$,

2. the evolution of the angular clustering of galaxies,

3. weak lensing tomography up to $z \sim 1$ and

4. distances and luminosities of supernovae in $0.3 \leq z \leq 0.8$

While observations and data analyses proceed by different working groups in the collaboration, simulations are also performed to validate the analysis tools and forecast results with higher confidence. In this work, we will make use of these simulations in order to test pipelines that estimate the angular correlation function of galaxies and how to extract cosmological information from a Bayesian analysis of the data.

\subsection{LSS cosmological observables}

This section introduces the basic cosmic fields whose correlations will be studied in the remaining of this work. Namely, the angular fluctuation of the density of galaxies in the Universe and the convergence and shear fields that characterize weak lensing phenomena.

This will be done from a point of view as general as possible, within a cosmological model based on first order (linear) scalar perturbations around a FLRW model. It will not be assumed from the beginning that the Universe is spatially flat, which will nonetheless be a particular and important case of the treatment here presented.

\subsubsection{Three and two-dimensional galaxy clustering}

In the context of galaxy surveys, one observes a particular window of the Universe, consisting of an angular mask of the observed area and a radial distribution of galaxies. In order to correct for spatially varying selection effects, we do not make direct use of observed galaxy number density $n_{\mathrm{g}}(\mathbf{x})$ but instead consider the dimensionless overdensity of galaxies $\delta_{\mathrm{g}}(\mathbf{x})=n_{\mathrm{g}}(\mathbf{x}) / \bar{n}_{\mathrm{g}}-1$, defined with respect to the mean number density $\bar{n}_{\mathrm{g}}$. 
In order to confront theoretical model predictions for the total mass distribution in the Universe against observational data, a relationship between the fluctuation fields of galaxies and the total matter becomes necessary. The problem appearing resides in the fact that luminous astrophysical objects, such as galaxies and quasars, are not direct (but biased) tracers of mass in the universe. A difference of the spatial distribution between luminous astrophysical objects and the total matter in the Universe has been indicated from a variety of observations. This difference is commonly referred to in the literature as the biasing effect. It is beyond the scope of this work to present a detailed discussion of galaxy bias modeling. However, some related ideas are presented below.

In common applications a linear biasing model is often assumed in which the fluctuation fields of galaxies $\delta_{\mathrm{g}}$ and total matter $\delta_{\mathrm{m}}$ are assumed to be deterministically related by

$$
\delta_{\mathrm{g}}=b_{\mathrm{g}} \delta_{\mathrm{m}}
$$

where the bias factor $b_{\mathrm{g}}$ fully determines the biasing effect. However, this modeling for the biasing is not based on any reasonable physical motivation. Note that, if the bias factor satisfies $b_{\mathrm{g}}>1$ everywhere the model should break down because values of the galaxy fluctuation field $\delta_{\mathrm{g}}$ below -1 are forbidden by definition, even in voids.

A better motivated and formulated approach to biasing effect is based on the biasing of density peaks in a Gaussian random field (see [31]). In this scheme the galaxy-galaxy and total matter two-point correlation functions, $\xi_{\mathrm{gg}}$ and $\xi_{\mathrm{mm}}$ respectively, are related in linear theory by

$$
\xi_{\mathrm{gg}}=b_{\mathrm{g}}^{2} \xi_{\mathrm{mm}},
$$

where the galaxy bias parameter is a constant independent of scale, i.e. a scale independent bias. Note that the relation (1.59) follows from (1.58), but the reverse is not true.

In this work a scale independent galaxy bias of the form (1.58) is used. This relation is assumed phenomenologically for a galaxy population and the bias factor is allowed to vary with the radial distance to galaxies, i.e. with redshift or cosmic time.

The key concept on the theoretical interpretation of angular clustering of galaxies is the field of projected galaxy density fluctuation onto the sky or projected galaxy fluctuation for short, denoted here $\delta_{\mathrm{g}}^{2 \mathrm{D}}(\mathbf{n})$. Here $\mathbf{n}=(\theta, \varphi)$ represents a particular direction (from the observer) on the sky. It is convenient to define it as a properly weighted marginalization of the total galaxy fluctuation on the observer past light-cone, $\delta_{\mathrm{g}}(\chi(z), \mathbf{n})$, over its redshift (radial) dependence (see e.g. [7, 8, 32]),

$$
\delta_{\mathrm{g}}^{2 \mathrm{D}}(\mathbf{n}):=\int_{0}^{\infty} \mathrm{d} z \phi^{\mathrm{g}}(z) \delta_{\mathrm{g}}(\chi(z), \mathbf{n})
$$

and should be interpreted as the total averaged galaxy fluctuation on a given direction on the sky. The marginalization kernel is the radial selection function of galaxies, $\phi^{\mathrm{g}}(z)$, which describes the selection of galaxies in redshift and depends on the kind of observation considered. It should take into account the sky coverage for the definition of the field, the method used to estimate the redshift of galaxies, spatially varying magnitude limits, etc.

The goal here is to derive the relation between the projected galaxy fluctuation $\delta_{\mathrm{g}}^{2 \mathrm{D}}$ and the total matter fluctuation today, $\delta_{0}(\chi, \theta, \varphi)$. This theoretical relation should then enable us to relate their statistical properties too. In order to find this relation it is necessary to deal with the proper relation between $\delta_{\mathrm{g}}$ and $\delta_{0}$, which is a non-trivial one.

The main complication resides in the fact that the peculiar velocity of a galaxy will cause it to appear shifted along the line-of-sight in redshift coordinates, an effect early 
studied by [33, 34]. In other words, the peculiar velocities of galaxies on top of their background Hubble flow introduce a radial anisotropic distortion in redshift-space (rs), the space of measured positions of galaxies. That is, the redshift distance of a body will be altered from its true distance, by its own peculiar velocity radially oriented from the observer. This deviation alters the apparent clustering of galaxies and, collectively, the effect is said to be the result of redshift space distortions (see Appendix C or classical references, e.g. [33, 34]).

In linear cosmological perturbation theory on a FLRW Universe, the observed redshiftspace fluctuation of galaxies $\delta_{\mathrm{g}}^{(\mathrm{rs})}$ is related to the total matter fluctuation today $\delta_{0}$ by the redshift space distortion operator, $\widehat{R}_{\mathrm{g}}$, an integro-differential operator, such that, on large scales (see Appendix $\mathrm{C}$ for a detailed discussion)

$$
\delta_{\mathrm{g}}^{(\mathrm{rs})}(\chi, \mathbf{n})=b_{\mathrm{g}}(z) G(z) \widehat{R}_{\mathrm{g}} \delta_{0}(\chi, \mathbf{n}),
$$

where $b_{\mathrm{g}}(z)$ represents the scale-invariant galaxy bias and $G(z)$ the growing mode of linear matter fluctuations dynamics.

A general treatment of redshift space distortions sourced by Doppler effect in the context of GR is presented on Appendix C. There, the general form for the redshift space distortion operator $\widehat{R}_{\mathrm{g}}$ is deduced assuming linear perturbation theory and linear scaleindependent biasing, and is valid for arbitrary radial separation between the observer and the galaxy (arbitrary galaxy redshift) and also arbitrary spatial curvature. The resulting operator is given by equation (C.36), reproduced here for completeness:

$$
\widehat{R}_{\mathrm{g}}=\widehat{1}+\beta_{\mathrm{g}}(z) \begin{cases}|K|\left[\partial_{\chi}+\alpha(\chi)\right] \partial_{\chi}\left(\nabla^{2}+3 K\right)^{-1} & K \neq 0 \\ {\left[\partial_{\chi}+\alpha(\chi)\right] \partial_{\chi}\left(\nabla^{2}\right)^{-1}} & K=0\end{cases}
$$

where $\widehat{1}$ represents the identity operator, $\beta_{\mathrm{g}}(z)$ (equation $(\mathrm{C} .37)$ ) is the standard redshiftspace distortion factor, determining the strength of the distortions, and $\alpha$ is an observationdependent function of the comoving distance given by equation (C.29).

Thus, the projected galaxy fluctuation can be written as the projection of the redshiftspace distorted fluctuation of matter today,

$$
\delta_{\mathrm{g}}^{2 \mathrm{D}}(\theta, \varphi)=\int_{0}^{\infty} \mathrm{d} z W^{\mathrm{g}}(z) \widehat{R}_{\mathrm{g}} \delta_{0}(\chi, \theta, \varphi)
$$

where the galaxy window function was introduced as

$$
W^{\mathrm{g}}(z):=\phi^{\mathrm{g}}(z) b_{\mathrm{g}}(z) G(z) .
$$

\subsubsection{Weak gravitational lensing}

In this section, the first Greek indices $\alpha, \beta, \delta \ldots$ run in 2,3 labeling coordinates used to cover the unit sphere $\mathbb{S}_{2}$. Unless mentioned otherwise, the system of usual spherical coordinates $(\theta, \varphi)$ is used, so that the metric is given by

$$
\left[g_{\alpha \beta}\right]=\left[\begin{array}{cc}
1 & 0 \\
0 & \sin ^{2}(\theta)
\end{array}\right]
$$

In the Born approximation, the deflection angle of a light ray by the large scale structure of the Universe, described using linear scalar perturbations around a FLRW background (see $\S A .3$ ), is given by a properly weighted projection on the sky of the $\mathbb{S}_{2}$-gradient 
of the Weyl or lensing gravitational potential, $\Psi_{W}:=(\Phi+\Psi) / 2$, evaluated along unperturbed radial null geodesics,

$$
\left(\theta-\theta_{0}, \sin \theta_{0}\left(\varphi-\varphi_{0}\right)\right)=\alpha^{\beta}=-2 \int_{0}^{r_{\mathrm{s}}} \mathrm{d} r \frac{f_{K}\left(r_{\mathrm{s}}-r\right)}{f_{K}\left(r_{\mathrm{s}}\right) f_{K}(r)} \nabla^{\beta} \Psi_{W}\left(\eta_{0}-r, r, \theta_{0}, \varphi_{0}\right),
$$

where the sub-indices "s" and "0" denote source (emission of the light ray) and observer (reception of the light ray events) and the function $f_{K}$ is given according to the radial components of the RW metric (equation (1.6)) in coordinates $x^{\mu}=(\eta, r, \theta, \varphi)$ by equation (1.7). The gradient on $\mathbb{S}_{2}$ on (1.65) can be interpreted as a perpendicular gradient to radial null geodesics in the FLRW background. An explicit computation of the deflection angle (1.65) is presented in Appendix D.

The lensing potential $\psi$ is introduced as

$$
\psi\left(\mathbf{n}_{0}\right)=2 \int_{0}^{r_{\mathrm{s}}} \mathrm{d} r \frac{f_{K}\left(r_{\mathrm{s}}-r\right)}{f_{K}\left(r_{\mathrm{s}}\right) f_{K}(r)} \Psi_{W}\left(\eta_{0}-r, r, \mathbf{n}_{0}\right),
$$

where $\mathbf{n}_{0}=\left(\theta_{0}, \varphi_{0}\right)$ is a direction on the observer sky, a point on $\mathbb{S}_{2}$, in other words, it is a scalar field on $\mathbb{S}_{2}$ such that its covariant gradient gives the deflection angle of light rays traveling from the source radial position, $r_{\mathrm{s}}$, to the observer position, $r_{0}=0$ (assumed to be the origin of radial coordinates without loss of generality), i.e.

$$
\alpha^{\beta}(\mathbf{n})=-\nabla^{\beta} \psi(\mathbf{n})
$$

Note that the lensing potential depends on the observation time $\eta_{0}$ and the source radial position $r_{\mathrm{s}}$.

The angular coordinates $\mathbf{n}_{0}$ in the deflection angle and the lensing potential expressions, (1.65) and (1.66), respectively, represent the undeflected angular position of the source, so that the application

$$
\left(\theta_{0}, \varphi_{0}\right) \mapsto\left(\theta_{\mathrm{s}}, \varphi_{\mathrm{s}}\right)=\left(\theta_{0}, \varphi_{0}\right)+\boldsymbol{\alpha}\left(\theta_{0}, \varphi_{0}\right)=\left(\theta_{0}, \varphi_{0}\right)-\nabla_{\perp} \psi(\mathbf{n})
$$

defines what in the literature is called the lens map. In other words, the lens map defines the application that takes the actual angular position of a source in the Universe to the observed deflected position.

The magnification matrix $\mathcal{A}$ is defined locally via the covariant derivative of the lens map (1.68), i.e. the Jacobian of the transformation

$$
\begin{aligned}
\mathcal{A}_{\alpha}{ }^{\beta} & =\delta_{\alpha}^{\beta}-\nabla_{\alpha} \nabla^{\beta} \psi\left(x^{\delta}\right) \\
& =\delta_{\alpha}{ }^{\beta}-2 \int_{0}^{r_{\mathrm{s}}} \mathrm{d} r \frac{f_{K}\left(r_{\mathrm{s}}-r\right)}{f_{K}\left(r_{\mathrm{s}}\right) f_{K}(r)} \nabla_{\alpha}\left[\nabla^{\beta} \Psi_{W}\left(\eta_{0}-r, r, x^{\delta}\right)\right] \\
& :=\left[\begin{array}{cc}
1-\kappa & 0 \\
0 & 1-\kappa
\end{array}\right]-\left[\begin{array}{cc}
\gamma_{1} & \gamma_{2} \\
\gamma_{2} & -\gamma_{1}
\end{array}\right]=\left[\begin{array}{cc}
1-\kappa-\gamma_{1} & -\gamma_{2} \\
-\gamma_{2} & 1-\kappa+\gamma_{1}
\end{array}\right] .
\end{aligned}
$$

Note that formally the magnification matrix is defined as a second-rank tensor on $\mathbb{S}_{2}$.

The magnification matrix $\mathcal{A}$ describes the deformation of a bundle of light rays incoming to an observer from a direction $\mathbf{n}\left(=x^{\delta}\right)$ in the sky, i.e. it describes how sources are locally deformed under the lens mapping. This is better understood in equation (1.69), where the separation into trace and trace-free parts is introduced in order to define the scalar fields $\kappa$ and $\gamma_{1,2}$. The former is the $\mathbb{S}_{2}$-divergence of the deflection angle,

$$
\kappa:=\frac{1}{2} \nabla_{\alpha} \nabla^{\alpha} \psi
$$


which defines the convergence field, the trace part of the magnification matrix. The latter is the corresponding trace-free part, defining the fields

$$
\begin{aligned}
& \gamma_{1}=\frac{1}{2}\left(\nabla_{2} \nabla^{2} \psi-\nabla_{3} \nabla^{3} \psi\right), \\
& \gamma_{2}=\nabla_{2} \nabla^{3} \psi=\nabla_{3} \nabla^{2} \psi,
\end{aligned}
$$

the components of the shear field, defined by convenience as the complex pair

$$
\gamma:=\gamma_{1}+i \gamma_{2}
$$

In absence of scattering, absorption or emission, the phase-space distribution function $f$ of photons satisfies Liouville's theorem. This implies that $f \propto \nu^{-3} I(\nu)$ is constant along null geodesics, where $\nu$ and $I(\nu)$ are the frequency and the specific intensity of the light. If the frequency is unchanged by the lensing mass distribution, $I(\nu)$ is constant and the flux from the source is changed only because the lens mapping changes the solid angle under which the source appears. As a result, lensing effect causes magnification $\mu$, given by

$$
\mu=|\operatorname{det}[\mathcal{A}]|^{-1} \text {. }
$$

The trace of the magnification matrix, $\operatorname{Tr}[\mathcal{A}]=2(1-\kappa)$, is a measure of the amount of focusing of light rays, while its traceless part, presented as the complex pair $\gamma$, measures shearing of lensed images. While the convergence is responsible for stretching a source isotropically under the lens mapping, the shear is responsible for its distortion. In fact, a circular source of unit radius is mapped into an elliptical image with semi-major and semi-minor axes $[11,10]$

$$
a=(1-\kappa-|\gamma|)^{-1}, \quad b=(1-\kappa+|\gamma|)^{-1} .
$$

This distortion allows the systematic detection of gravitational lensing. The relative axis ratio of elliptically distorted images, the ellipticity $\epsilon$, is the so-called reduced shear,

$$
\epsilon=\frac{a-b}{a+b}=\frac{|\gamma|}{1-\kappa}=|g|
$$

Applying the local definition of the convergence to the lensing potential on the canonical basis on the sphere we find

$$
\kappa(\theta, \varphi)=\int_{0}^{r_{\mathrm{s}}} \mathrm{d} r \frac{f_{K}\left(r_{\mathrm{s}}-r\right)}{f_{K}\left(r_{\mathrm{s}}\right) f_{K}(r)} \nabla_{\alpha} \nabla^{\alpha} \Psi_{W}\left(\eta_{0}-r, r, \theta, \varphi\right) .
$$

The main goal here is to derive a relation between the lensing convergence field $\kappa$ and the total matter fluctuation today, in an analogous way to what was done for the angular fluctuation of galaxies in $\S 1.4 .1$ (equation (1.62)). The key insight to relate these two fields is the fact that one can augment the two-dimensional Laplacian in equation (1.76) by a term of derivatives along the line-of-sight. In fact, the 3D Laplacian on the constant time hypersurfaces $\Sigma_{t}$ can be expanded as (by using e.g. equation (B.2)):

$$
\nabla^{2} \Psi_{W}=\frac{1}{f_{K}^{2}(r)}\left[\frac{\partial}{\partial r}\left(f_{K}^{2}(r) \frac{\partial \Psi_{W}}{\partial r}\right)+\nabla_{\alpha} \nabla^{\alpha} \Psi_{W}\right]
$$

Notice that this Laplacian is related to the energy density according to EFE. For the case of first order scalar perturbations (see Appendix A), and in the absence of anisotropic 
stress, the Weyl potential reduces to the gauge invariant potential $\hat{\Phi}$ (equation (A.38)), which in turns is sourced by the gauge invariant fluctuations in the matter according to the first-order perturbed EFE (equation (A.53c)),

$$
\left(\nabla^{2}+3 K\right) \Psi_{W}=4 \pi G a^{2} \bar{\rho} \delta
$$

where $\bar{\rho}$ and $\delta$ are associated only with pressureless matter.

One can then consider the geometrically weighted integration along the line of sight of equation (1.78), where by the geometrical weight one refers to the kernel in the integral giving the lensing convergence (equation (1.76)) for the $\mathbb{S}_{2}$-Laplacian of the Weyl potential. Assuming the Weyl potential to be localized, the terms involving derivatives along the line-of-sight direction $r$ vanish after the line-of-sight integration in equation (1.76) or contribute with terms that average to zero in the limit of interest. Thus, one can conclude that the convergence is a suitably scaled and geometrically weighed surface-mass density of matter inhomogeneities:

$$
\kappa(\theta, \varphi)=4 \pi G a^{2} \bar{\rho}_{\mathrm{m}} \int_{0}^{r_{\mathrm{s}}} \mathrm{d} r \frac{f_{K}\left(r_{\mathrm{s}}-r\right) f_{K}(r)}{f_{K}\left(r_{\mathrm{s}}\right)} \delta_{\mathrm{m}}\left(\eta_{0}-r, r, \theta, \varphi\right)
$$

or, by introducing the background evolution of matter, $4 \pi G a^{2} \bar{\rho}=3 H_{0}^{2} \Omega_{\mathrm{m}} / 2 a$, and the time evolution of matter fluctuations,

$$
\kappa(\theta, \varphi)=\frac{3 H_{0}^{2}}{2} \Omega_{\mathrm{m}} \int_{0}^{r_{\mathrm{s}}} \mathrm{d} r \frac{f_{K}\left(r_{\mathrm{s}}-r\right) f_{K}(r)}{f_{K}\left(r_{\mathrm{s}}\right)} \frac{G(z(r))}{a(r)} \delta_{0}(r, \theta, \varphi),
$$

where $\delta_{0}$ is the density contrast of total matter in the Universe today. The equation (1.80) gives the convergence field observed at $z_{0}=0\left(r_{0}=0\right)$ associated to localized sources at $z_{\mathrm{s}}\left(r_{\mathrm{s}}=r\left(z_{\mathrm{s}}\right)\right)$ generated by the lensing effect on light rays deflected by total intervening matter all the way from the source to the observer.

To have an insight on this result, consider the contribution to the convergence from a single radially-located lens modeled by the matter fluctuation (today) of the form

$$
\delta_{0}^{\mathrm{L}}(r, \theta, \varphi)=\frac{\Sigma_{0}^{\mathrm{L}}(\theta, \varphi)}{\bar{\rho}_{\mathrm{m} 0}} \delta_{\mathrm{D}}^{(1)}\left(r-r_{\mathrm{L}}\right)
$$

where $\Sigma_{0}^{\mathrm{L}}$ is the lens surface mass density today and $r_{\mathrm{L}}=r\left(z_{\mathrm{L}}\right)<r_{\mathrm{s}}$ is its radial distance to the observer (given by its redshift, $z_{\mathrm{L}}$ ). The convergence field contribution from this single lens is then given by

$$
\begin{aligned}
\kappa^{\mathrm{L}}(\theta, \varphi) & =4 \pi G \frac{f_{K}\left(r_{\mathrm{s}}-r_{\mathrm{L}}\right) f_{K}\left(r_{\mathrm{L}}\right)}{f_{K}\left(r_{\mathrm{s}}\right)} \frac{G\left(z_{\mathrm{L}}\right)}{a_{\mathrm{L}}} \Sigma_{0}^{\mathrm{L}}(\theta, \varphi) \\
& =\frac{1}{\Sigma_{\mathrm{cr}}} \frac{G\left(z_{\mathrm{L}}\right)}{a_{\mathrm{L}}} \Sigma_{0}^{\mathrm{L}}(\theta, \varphi),
\end{aligned}
$$

where the critical surface density $\Sigma_{\text {cr }}$ was introduced

$$
\Sigma_{\mathrm{cr}}:=\frac{1}{4 \pi G} \frac{f_{K}\left(r_{\mathrm{s}}\right)}{f_{K}\left(r_{\mathrm{s}}-r_{\mathrm{L}}\right) f_{K}\left(r_{\mathrm{L}}\right)} .
$$

Note that $\Sigma_{0}^{\mathrm{L}}(\theta, \varphi) G\left(z_{\mathrm{L}}\right) / a_{\mathrm{L}}$ is the surface mass density of the single lens at the moment it deflects the light ray. 
For a redshift distribution of sources, $p_{z}(z) \mathrm{d} z=p_{r}(r) \mathrm{d} r$, the convergence field should be given by the averaged superposition of the effect of all sources over the (normalized) source-distance distribution,

$$
\kappa(\theta, \varphi)=\int_{0}^{r_{\mathrm{H}}} \mathrm{d} r p_{r}(r) \kappa(\theta, \varphi, r)
$$

where $r_{H}$ is the comoving horizon distance, obtained from the comoving distance-redshift relation, $r(z)$, letting $z \rightarrow \infty$.

Computing explicitly,

$$
\begin{aligned}
\kappa(\theta, \varphi) & =\int_{0}^{r_{\mathrm{H}}} \mathrm{d} r p_{r}(r) \kappa(\theta, \varphi, r) \\
& =\frac{3}{2} \frac{H_{0}^{2}}{c^{2}} \Omega_{\mathrm{m}} \int_{0}^{r_{\mathrm{H}}} \mathrm{d} r \int_{0}^{r} \mathrm{~d} r^{\prime} p_{r}(r) \frac{f_{K}\left(r-r^{\prime}\right) f_{K}\left(r^{\prime}\right)}{f_{K}(r)} \frac{G\left(z\left(r^{\prime}\right)\right)}{a\left(r^{\prime}\right)} \delta_{0}\left(r^{\prime}, \theta, \varphi\right),
\end{aligned}
$$

changing appropriately the order of integration,

$$
\kappa(\theta, \varphi)=\frac{3}{2} \frac{H_{0}^{2}}{c^{2}} \Omega_{\mathrm{m}} \int_{0}^{r_{\mathrm{H}}} \mathrm{d} r^{\prime} \int_{r^{\prime}}^{r_{\mathrm{H}}} \mathrm{d} r p_{r}(r) \frac{f_{K}\left(r-r^{\prime}\right) f_{K}\left(r^{\prime}\right)}{f_{K}(r)} \frac{G\left(z\left(r^{\prime}\right)\right)}{a\left(r^{\prime}\right)} \delta_{0}\left(r^{\prime}, \theta, \varphi\right) .
$$

At this point it is useful to introduce the lensing efficiency function,

$$
g\left(r^{\prime}\right):=\int_{r^{\prime}}^{r_{\mathrm{H}}} \mathrm{d} r p_{r}(r) \frac{f_{K}\left(r-r^{\prime}\right)}{f_{K}(r)}
$$

In terms of the efficiency function one can define the lensing window function, which relates the density contrast and convergence fields via a projection onto the sky,

$$
\begin{gathered}
\kappa(\theta, \varphi)=\int_{0}^{\infty} \mathrm{d} z W^{\kappa}(z) \delta_{0}(r, \theta, \varphi) \\
W^{\kappa}(z):=\frac{3}{2} \frac{H_{0}^{2}}{c^{2}} \Omega_{\mathrm{m}} \frac{c}{H(z)} G(z)(1+z) g(r(z)) f_{K}(r(z)) \\
=\frac{3}{2} \frac{H_{0}}{c} \Omega_{\mathrm{m}} \frac{G(z)(1+z)}{E(z)} g(r(z)) f_{K}(r(z)),
\end{gathered}
$$

where $E(z)$ describes the Universe expansion history according to the Friedmann equation (1.17).

It is interesting to note that in the particular case in which the sources are at a fixed redshift, $z_{\mathrm{s}}$, we can describe the distribution using $p_{r}(r)=\delta_{D}^{(1)}\left(r-r_{\mathrm{s}}\right)$. Then the efficiency function reduces to

$$
g\left(r^{\prime}\right):=\int_{r^{\prime}}^{r_{\mathrm{H}}} \mathrm{d} r \delta_{D}^{(1)}\left(r-r_{\mathrm{s}}\right) \frac{f_{K}\left(r-r^{\prime}\right)}{f_{K}(r)}=\frac{f_{K}\left(r_{\mathrm{s}}-r^{\prime}\right)}{f_{K}\left(r_{\mathrm{s}}\right)} \Theta\left(r_{\mathrm{s}}-r^{\prime}\right),
$$

where $\Theta$ is the Heaviside step function, the efficiency function for punctual lens and source systems. Thus, one can interprete the equation (1.85) as a generalization of the lensing efficiency factor $f_{K}\left(r_{\mathrm{s}}-r_{\mathrm{L}}\right) / f_{K}\left(r_{\mathrm{s}}\right)$, quotient between the angular diameter distances between lens and source, and source and observer, of the punctual lens and puntual source systems [11]. 


\section{Chapter 2}

\section{Two-point statistics in the Universe}

The current model for the large scale structure of the Universe is based on the concepts depicted on the previous chapter. The observed distribution of the matter/energy content of the Universe then results from the growth of primordial seed fluctuations, which may be generated by quantum fluctuations during inflationary period in the early Universe, and were amplified by gravitational instability phenomena, which is formally described by cosmological perturbation theory [27, 4]. Thus, a first problem appears in Cosmology coming from two reasons: (a) one does not have access to the initial conditions of the evolution of perturbations, i.e., the primordial fluctuations and (b) the time-scale for cosmological evolution is too large, thus is not possible to follow the evolution of single systems. The resulting problem is that, consequently, fluctuations around a perturbed FLRW background should be treated as random variables. The observable universe is thus modeled as a stochastic realization of a statistical ensemble of possibilities. In this context observations should be used to determine statistical properties of such fluctuations. A perturbative variable, as e.g. the fluctuations in matter density $\delta(\mathbf{x})$ at some fixed time, is associated with an ensemble of random functions, each with a probability assigned to it. This is the notion of a random field, an application that takes points from some space, as e.g. $\quad \Sigma_{t}$ (the constant time hypersurfaces of FLRW models) or $\mathbb{S}_{2}$ (the unit sphere representing the observer sky), and assigns a random variable. It is beyond the scope of this work to discuss the concept of a random field in depth, and we refer the reader to e.g. the discussion of $[3,27]$ and references therein.

A second problem arising in this context is related with an intrinsic limitation in Cosmology. As long as there is only one Universe to observe one has a single realization of the stochastic process associated with the fluctuations whose consequences one wants to observe. Therefore, it is impossible to measure ensemble averages or expectation values as physicists are accustomed to, that is, in a repeatable and controlled laboratory experiment. However, What can be done observing a fluctuation on a given scale $r$ is to average over many distinct regions of typical size $r$. Then, an ergodic-type hypothesis should be considered in order to replace the (desired) ensemble average by a (possible) spatial average over these regions. Such an hypothesis is known in the literature as the fair sample hypothesis, stating that the finite part of the Universe accessible to observations is a fair sample of the whole Universe (see e.g. $\S 6.3$ of [27]). In principle, this is reasonable when the scale is much less than the observable Universe $\left(r \ll H_{0}^{-1}\right)$. But on larger scales it is impossible to average over many volumes and thus the measured value could be far from the ensemble average. This is called the cosmic variance problem.

The most basic statistical quantity that one can construct with a fluctuation field is 
the two-point correlation function. The two-point correlation function of a perturbation variable $\delta,\left\langle\delta(\mathbf{x}) \delta\left(\mathbf{x}^{\prime}\right)\right\rangle$, is defined as the average over the ensemble (incorporating the probability distribution of $\delta$ considered as a random field). In order to have a physical insight on this quantity, consider that the number density of galaxies in the Universe is given in terms of a fluctuation field as $n_{\mathrm{g}}(\mathbf{x})=\bar{n}_{\mathrm{g}}\left(1+\delta_{\mathrm{g}}(\mathbf{x})\right)$, where $\bar{n}_{\mathrm{g}}$ is the total spatial average number density (independent of the position) and $\delta_{\mathrm{g}}$ represents the fluctuation (position-dependent). Therefore, the number of galaxy pairs separated by a distance $r$ (comoving) on a direction specified by the unit vector $\hat{\mathbf{n}}$ can be expressed as

$$
\mathrm{d} N_{\text {pair }}(r, \hat{\mathbf{n}})=n_{\mathrm{g}}(\mathbf{x}) \mathrm{d} V_{1} n_{\mathrm{g}}(\mathbf{x}+r \hat{\mathbf{n}}) \mathrm{d} V_{2},
$$

where $\mathrm{d} V_{1}$ and $\mathrm{d} V_{2}$ are volume elements around $\mathbf{x}$ and $\mathbf{x}+r \hat{\mathbf{n}}$ respectively, or equivalently, by introducing the fluctuation field, as

$$
\mathrm{d} N_{\text {pair }}(r, \hat{\mathbf{n}})=\bar{n}_{\mathrm{g}}^{2}\left[1+\delta_{\mathrm{g}}(\mathbf{x})+\delta_{\mathrm{g}}(\mathbf{x}+r \hat{\mathbf{n}})+\delta_{\mathrm{g}}(\mathbf{x}) \delta_{\mathrm{g}}(\mathbf{x}+r \hat{\mathbf{n}})\right] \mathrm{d} V_{1} \mathrm{~d} V_{2} .
$$

After averaging over all possible volume elements and considering the $\delta_{\mathrm{g}}$ field as a fluctuation in the sense of having zero mean, $\left\langle\delta_{\mathrm{g}}\right\rangle=0$, the expected number of galaxy pairs separated by a distance $r$ on the direction $\hat{\mathbf{n}}$ is given by

$$
\left\langle\mathrm{d} N_{\text {pairs }}(r, \hat{\mathbf{n}})\right\rangle=\bar{n}_{\mathrm{g}}^{2}\left[1+\left\langle\delta_{\mathrm{g}}(\mathbf{x}) \delta_{\mathrm{g}}(\mathbf{x}+r \hat{\mathbf{n}})\right\rangle\right] \mathrm{d} V_{1} \mathrm{~d} V_{2} .
$$

When there is no fluctuation on the number density of galaxies, the expected number of pairs separated by a distance $r$ on the direction $\hat{\mathbf{n}}$ is given by the squared spatial average number of galaxies, thus, independent of the position. When the fluctuation is taken into account, the number of pairs is position dependent and consequently, it appears an excess (lack) of probability of finding a pair of galaxies depending on their separation, $r$ and $\hat{\mathbf{n}}$; this excess (lack) probability is quantified by the two-point correlation function, defined as

$$
\xi_{\mathrm{g}}(r, \hat{\mathbf{n}})=\left\langle\delta_{\mathrm{g}}(\mathbf{x}) \delta_{\mathrm{g}}(\mathbf{x}+r \hat{\mathbf{n}})\right\rangle .
$$

Given a catalog of $N$ galaxies with their corresponding positions, one can think on a natural estimator for the probability of finding a pair of galaxies separated by a distance $r$ on the $\hat{\mathbf{n}}$ direction. When a catalog of galaxies is provided frequentist statistics techniques can be used to estimate this quantity as the ratio between the number of pairs separated by a distance $r$ on direction $\hat{\mathbf{n}}$ in the catalog $P_{D D}(r, \hat{\mathbf{n}})$ (correctly normalized by the number of possible pairs $N(N-1)$, see equations (2.26)) and the number of pairs with the same characteristics on a constructed catalog of randomly distributed galaxies $P_{R R}(r, \hat{\mathbf{n}})$ (equations (2.26)),

$$
\frac{D D(r, \hat{\mathbf{n}})}{R R(r, \hat{\mathbf{n}})}=\frac{\left\langle\mathrm{d} N_{\text {pair }}(r, \hat{\mathbf{n}})\right\rangle}{\bar{n}_{\mathrm{g}}^{2} \mathrm{~d} V_{1} \mathrm{~d} V_{2}}=1+\widehat{\xi}_{\mathrm{g}}(r, \hat{\mathbf{n}}) .
$$

This statistical estimator for the two-point correlation function is known as the natural estimator and will be discussed on $\S 2.1 .2$. Hereinafter the hat-notation $\widehat{\cdot}$ will denote a statistical estimator for a quantity.

The perturbative quantities described as random fields are usually assumed to be statistically homogeneous and isotropic, i.e. invariant under translations and rotations. For the two-point correlation function the statistical homogeneity assumption translates into the condition $\langle\delta(\mathbf{x}-\mathbf{a}) \delta(\mathbf{x}+r \hat{\mathbf{n}}-\mathbf{a})\rangle=\langle\delta(\mathbf{x}) \delta(\mathbf{x}+r \hat{\mathbf{n}})\rangle$ for all $\mathbf{a}$, which implies that the correlation function should depend only on the separation between the points, $r \hat{\mathbf{n}}$, i.e., $\xi_{\delta}(r, \hat{\mathbf{n}})=\xi_{\delta}(r \hat{\mathbf{n}})$. The statistical isotropy assumption on the other hand translates 
into the condition $\xi_{\delta}(r R[\hat{\mathbf{n}}])=\xi_{\delta}(r \hat{\mathbf{n}})$, where $R$ represents an arbitrary spatial rotation, which implies that the correlation function should depend only on the absolute value of the separation between the two points $r$, and therefore can be written as

$$
\xi_{\delta}(r)=\langle\delta(\mathbf{x}) \delta(\mathbf{x}+r \hat{\mathbf{n}})\rangle
$$

so that $\xi_{\delta}$ does not depend on the position $\mathbf{x}$ or the (unit) direction $\hat{\mathbf{n}}$.

\subsection{Configuration space}

In this section some results on the two-point correlation function of random ields in configuration space are presented. We begin by considering the relation between the two-point correlation function and the power spectrum of a random field defined on the constant time hypersurfaces of a FLRW model. Explicit relations between these quantities are found depending on the sign of the spatial curvature considered. In order to do so, we closely follow the treatment in [35].

A brief review of usual techniques to measure the correlation function is presented, with special focus on pair counts-based estimators. A discussion on the bias of different estimators is presented showing its deep relation with the uncertainty in the mean density of a quantity from measurements. The section ends with a discussion on the comparison of different pair counting-based estimators. All this review is based on [36] and the references therein, which can be consulted for further discussions.

\subsubsection{Correlation function and power spectrum}

Consider a scalar fluctuation field $\delta$ defined at some time on an FLRW model. The field $\delta$ is a scalar defined on the constant time hypersurfaces of the model $\Sigma_{t}$, i.e. $\delta=\delta(\chi, \theta, \varphi)$, for example, the fluctuation on the total matter or the number density of galaxies in the Universe, and therefore can be expanded in scalar harmonic modes according to spacetime spatial curvature as (a review of the scalar harmonic decomposition is presented in Appendix B)

$$
\delta(\chi, \theta, \varphi)= \begin{cases}\sum_{\ell=0}^{\infty} \sum_{m=-\ell}^{\ell} \int_{0}^{\infty} \frac{\mathrm{d} \nu \nu^{2}}{2 \pi^{2}} \delta_{\ell m}(\nu) \widehat{X}_{\ell}^{(-)}(\chi, \nu) Y_{\ell}^{m}(\theta, \varphi) & K<0 \\ \sum_{\ell=0}^{\infty} \sum_{m=-\ell}^{\ell} \int_{0}^{\infty} \frac{\mathrm{d} \nu \nu^{2}}{2 \pi^{2}} \delta_{\ell m}(\nu) \widehat{X}_{\ell}^{(0)}(\chi, \nu) Y_{\ell}^{m}(\theta, \varphi) & K=0 \\ \sum_{\ell=0}^{\infty} \sum_{m=-\ell}^{\ell} \sum_{\nu=3}^{\infty} \frac{\nu^{2}}{2 \pi^{2}} \delta_{\ell m}(\nu) \widehat{X}_{\ell}^{(+)}(\chi, \nu) Y_{\ell}^{m}(\theta, \varphi) & K>0\end{cases}
$$

where the functions $\widehat{X}_{\ell}(\nu)$ represent the radial harmonic modes on the constant time hypersurfaces $\Sigma_{t}$ (equations (B.27)).

Note that for a spatially closed Universe model $(K>0)$ there are special features. As discussed in Appendix B the spectrum of the Laplace operator for this case is discrete. In addition, it can be seen that in the case in which one considers fluctuations in the total matter field the $\nu=2$ corresponds to a pure gauge mode. This follows from the Poisson equation (A.53c), because combined with the definition of the adimensional eigenvalue 
for the Laplace operator $\nu$ (equations (B.5)) it is inconsistent with the positivity of $\bar{\rho}_{\mathrm{m}} \delta_{\mathrm{m}}$ $[35,23]$. Since our goal is to derive the relation between the two-point correlation and the power spectrum for the matter density fluctuations in equations (2.7) the $\nu=2$ mode for $K>0$ was not considered.

The inverse expansions giving the harmonic modes $\delta_{\ell m}(\nu)$ are (see $\S$ B.1)

$$
\delta_{\ell m}(\nu)= \begin{cases}4 \pi \int \mathrm{d} \chi \sinh ^{2}(\chi) \int \mathrm{d}^{2} \Omega \delta(\chi, \theta, \varphi) \widehat{X}_{\ell}^{(-)}(\chi, \nu) Y_{\ell}^{m *}(\theta, \varphi), & \text { for } K<0 \\ 4 \pi \int \mathrm{d} \chi \chi^{2} \int \mathrm{d}^{2} \Omega \delta(\chi, \theta, \varphi) \widehat{X}_{\ell}^{(0)}(\chi, \nu) Y_{\ell}^{m *}(\theta, \varphi), & \text { for } K=0 \\ 4 \pi \int \mathrm{d} \chi \sin ^{2}(\chi) \int \mathrm{d}^{2} \Omega \delta(\chi, \theta, \varphi) \widehat{X}_{\ell}^{(+)}(\chi, \nu) Y_{\ell}^{m *}(\theta, \varphi), & \text { for } K>0 .\end{cases}
$$

One can interpret the last two set of equations providing generalizations of the usual Fourier decomposition in three dimensional Euclidean space. This is because the harmonic expansion of equation (2.7) for $\delta$ in the Euclidean case $(K=0)$ is equivalent to the 3D Fourier expansion

$$
\delta(\mathbf{x})=\delta(\chi, \theta, \varphi)=\frac{1}{(2 \pi)^{3}} \int \mathrm{d}^{3} \mathbf{k} \delta(\mathbf{k}) e^{i \mathbf{k} \cdot \mathbf{x}}
$$

because of the Rayleigh plane wave expansion [37]

$$
e^{i \mathbf{k} \cdot \mathbf{x}}=4 \pi \sum_{\ell=0}^{\infty} \sum_{m=-\ell}^{\ell} i^{\ell} j_{\ell}(k r) Y_{\ell}^{m *}\left(\theta_{\mathbf{k}}, \varphi_{\mathbf{k}}\right) Y_{\ell}^{m}\left(\theta_{\mathbf{x}}, \varphi_{\mathbf{x}}\right),
$$

where for the flat case $|\mathbf{x}|=\chi=r$ (equations (1.5)). Substituting equation (2.10) into (2.9), using spherical coordinates in Fourier space, $\mathrm{d}^{3} \mathbf{k}=k^{2} \mathrm{~d} k \mathrm{~d}^{2} \Omega_{k}=k^{2} \sin \left(\theta_{\mathbf{k}}\right) \mathrm{d} k \mathrm{~d} \theta_{\mathbf{k}} \mathrm{d} \varphi_{\mathbf{k}}$ and remembering that for the Euclidean case $\nu=k$ (equations (B.5)) one ends up with the relation between the Fourier modes $\delta(\mathbf{k})$ and the spherical harmonic modes $\delta_{\ell m}(k)$ :

$$
\delta_{\ell m}(k)=i^{\ell} \int \mathrm{d}^{2} \Omega_{\mathbf{k}} \delta(\mathbf{k}) Y_{\ell}^{m *}\left(\theta_{\mathbf{k}}, \varphi_{\mathbf{k}}\right)
$$

which is, due to the normalization convention of the harmonic decomposition (equation (2.7)), a usual spherical harmonic decomposition in Fourier space.

Since according to the assumptions of statistical homogeneity and isotropy the twopoint correlation function must depend only on the spatial separation of the two points, using spatial coordinates $x^{i}=(\chi, \theta, \varphi)$, one can compute the two-point correlation function as $\xi(\chi)=\langle\delta(0, \theta, \varphi) \delta(\chi, \theta, \varphi)\rangle$. Therefore,

$$
\begin{aligned}
\xi(\chi)= & \sum_{\ell, m} \sum_{\ell^{\prime}, m^{\prime}} \int \frac{\mathrm{d} \nu \nu^{2}}{2 \pi^{2}} \int \frac{\mathrm{d} \nu^{\prime} \nu^{\prime 2}}{2 \pi^{2}} \widehat{X}_{\ell}^{(-, 0)}(0, \nu) \widehat{X}_{\ell^{\prime}}^{(-, 0)}\left(\chi, \nu^{\prime}\right) Y_{\ell}^{m *}(\theta, \varphi) Y_{\ell^{\prime}}^{m^{\prime}}(\theta, \varphi) \\
& \times\left\langle\delta_{\ell m}^{*}(\nu) \delta_{\ell^{\prime} m^{\prime}}\left(\nu^{\prime}\right)\right\rangle,
\end{aligned}
$$

for $K \leq 0$ and

$$
\begin{aligned}
\xi(\chi)= & \sum_{\ell, m} \sum_{\ell^{\prime}, m^{\prime}} \sum_{\nu=3}^{\infty} \frac{\nu^{2}}{2 \pi^{2}} \sum_{\nu^{\prime}=3}^{\infty} \frac{\nu^{\prime 2}}{2 \pi^{2}} \widehat{X}_{\ell}^{(+)}(0, \nu) \widehat{X}_{\ell^{\prime}}^{(+)}\left(\chi, \nu^{\prime}\right) Y_{\ell}^{m *}(\theta, \varphi) Y_{\ell^{\prime}}^{m^{\prime}}(\theta, \varphi) \\
& \times\left\langle\delta_{\ell m}^{*}(\nu) \delta_{\ell^{\prime} m^{\prime}}\left(\nu^{\prime}\right)\right\rangle,
\end{aligned}
$$


for $K>0$.

In general, the correlation of the spherical harmonic modes defines the 3D spherical power spectrum $S_{\ell}\left(k, k^{\prime}\right)$ which has been used in the literature for the Euclidean case, see e.g. $[38,39,40]$. Here the natural generalization for open and closed FLRW Universe models is presented. According to the normalization conventions

$$
\left\langle\delta_{\ell m}^{*}(\nu) \delta_{\ell^{\prime} m^{\prime}}\left(\nu^{\prime}\right)\right\rangle=(2 \pi)^{3} \delta_{\ell \ell^{\prime}} \delta_{m m^{\prime}} S_{\ell}\left(\nu, \nu^{\prime}\right),
$$

For a statistically homogeneous and isotropic field $\delta$ the spherical power spectrum should be rotationally and translationally invariant, so that it reduces to the 3D power spectrum $S(\nu)[35]$

$$
S_{\ell}\left(\nu, \nu^{\prime}\right)= \begin{cases}\frac{\delta_{\mathrm{D}}\left(\nu-\nu^{\prime}\right)}{\nu^{2}} S(\nu) & K \leq 0, \\ \frac{\delta_{\nu \nu^{\prime}}}{\nu^{2}} S(\nu) & K>0 .\end{cases}
$$

In order to find the relation between $\xi(\chi)$ and $S(\nu)$ for statistically homogeneous and isotropic fields one can substitute equation (2.14) into (2.12). All cases are analogous, consider e.g. the case of an open universe $(K<0)$,

$$
\begin{aligned}
\xi(\chi)= & \sum_{\ell, m} \sum_{\ell^{\prime}, m^{\prime}} \int \frac{\mathrm{d} \nu \nu^{2}}{2 \pi^{2}} \int \frac{\mathrm{d} \nu^{\prime} \nu^{\prime 2}}{2 \pi^{2}} \widehat{X}_{\ell}^{(-)}(0, \nu) \widehat{X}_{\ell^{\prime}}^{(-)}\left(\chi, \nu^{\prime}\right) Y_{\ell}^{m *}(\theta, \varphi) Y_{\ell^{\prime}}^{m^{\prime}}(\theta, \varphi)\left\langle\delta_{\ell m}^{*}(\nu) \delta_{\ell^{\prime} m^{\prime}}\left(\nu^{\prime}\right)\right\rangle \\
= & (2 \pi)^{3} \sum_{\ell, m} \sum_{\ell^{\prime}, m^{\prime}} \int \frac{\mathrm{d} \nu \not^{\not 2}}{2 \pi^{2}} \int \frac{\mathrm{d} \nu^{\prime} \nu^{\prime 2}}{2 \pi^{2}} \widehat{X}_{\ell}^{(-)}(0, \nu) \widehat{X}_{\ell^{\prime}}^{(-)}\left(\chi, \nu^{\prime}\right) Y_{\ell}^{m *}(\theta, \varphi) Y_{\ell^{\prime}}^{m^{\prime}}(\theta, \varphi) \\
& \delta_{\ell \ell^{\prime}} \delta_{m m^{\prime}} \frac{\delta\left(\nu-\nu^{\prime}\right)}{\not^{2}} S(\nu) \\
= & 4 \pi \sum_{\ell, m} \int \frac{\mathrm{d} \nu \nu^{2}}{2 \pi^{2}} \widehat{X}_{\ell}^{(-)}(0, \nu) \widehat{X}_{\ell}^{(-)}(\chi, \nu) Y_{\ell}^{m *}(\theta, \varphi) Y_{\ell}^{m}(\theta, \varphi) S(\nu),
\end{aligned}
$$

so that, by using the property of scalar harmonic modes that at $\chi=0$ the only non-null mode is the lowest one $(\ell=0), \widehat{X}_{\ell}(\chi=0, \nu)=\delta_{\ell 0}$ (equation (B.30)), one can easily perform the sums over $\ell$ and $m$ to have

$$
\begin{aligned}
\xi(\chi) & =4 \pi \sum_{\ell, m} \int \frac{\mathrm{d} \nu \nu^{2}}{2 \pi^{2}} \delta_{\ell 0} \widehat{X}_{\ell}^{(-)}(\chi, \nu) Y_{\ell}^{m *}(\theta, \varphi) Y_{\ell}^{m}(\theta, \varphi) S(\nu) \\
& =4 \pi \int \frac{\mathrm{d} \nu \nu^{2}}{2 \pi^{2}} \widehat{X}_{0}^{(-)}(\chi, \nu) Y_{0}^{0 *}(\theta, \varphi) Y_{0}^{0}(\theta, \varphi) S(\nu) \\
& =\int \frac{\mathrm{d} \nu \nu^{2}}{2 \pi^{2}} X_{0}^{(-)}(\chi, \nu) S(\nu),
\end{aligned}
$$

where in the last line the properties $Y_{0}^{0 *}(\theta, \varphi) Y_{0}^{0}(\theta, \varphi)=\left|Y_{0}^{0}(\theta, \varphi)\right|^{2}=(4 \pi)^{-1}$ and $\widehat{X}_{0}^{(-)}=$ $X_{0}^{(-)}$(see Appendix B) for the Spherical Harmonics and the scalar harmonic modes were used.

Thus one finally arrives to the following closed relations

$$
\xi(\chi)= \begin{cases}\int_{0}^{\infty} \frac{\mathrm{d} \nu \nu^{2}}{2 \pi^{2}} X_{0}^{(-, 0)}(\chi, \nu) S(\nu), & K \leq 0 \\ \sum_{\nu=3}^{\infty} \frac{\nu^{2}}{2 \pi^{2}} X_{0}^{(+)}(\chi, \nu) S(\nu) & K>0 .\end{cases}
$$


For the $K \neq 0$ cases one can consider the change of variables from $\nu \rightarrow k$ according to equations (B.5), i.e. the change from the adimensional harmonic variable $\nu$ to the fully dimensional one $k\left([k]=\right.$ length $\left.^{-1}\right)$. This change is useful to compare the results presented here to those commonly presented in the literature for the Euclidean case. Consider again the open universe model $(K<0)$ for which the change is given by $k^{2}:=-K \nu^{2}=|K| \nu^{2}$, then

$$
\begin{aligned}
\xi(\chi) & =\int_{0}^{\infty} \frac{\mathrm{d} k k^{2}}{2 \pi^{2}} X_{0}^{(-)}\left(\chi,|K|^{-1 / 2} k\right) \frac{S\left(|K|^{-1 / 2} k\right)}{|K|^{3 / 2}} \\
& =\int_{0}^{\infty} \frac{\mathrm{d} k k^{2}}{2 \pi^{2}} X_{0}^{(-)}\left(\chi,|K|^{-1 / 2} k\right) P(k),
\end{aligned}
$$

where the fully dimensional power spectrum $P(k)\left([P]=\right.$ length $\left.^{3}\right)$ was introduced as

$$
P(k):=\frac{S\left(|K|^{1 / 2} k\right)}{|K|^{3 / 2}} .
$$

The closed model $(K>0)$ is analogous, the change of variable is given by $k^{2}:=K \nu^{2}=$ $|K| \nu^{2}$, then

$$
\begin{aligned}
\xi(\chi) & =\sum_{k \in\left\{3|K|^{1 / 2}, 4|K|^{1 / 2}, \ldots\right\}} \frac{\mathrm{d} k k^{2}}{2 \pi^{2}} X_{0}^{(+)}\left(\chi,|K|^{-1 / 2} k\right) \frac{S\left(|K|^{-1 / 2} k\right)}{|K|} \\
& =\sum_{i=1}^{\infty} \frac{k_{i}^{2}}{2 \pi^{2}} X_{0}^{(+)}\left(\chi,|K|^{-1 / 2} k_{i}\right)|K|^{1 / 2} P\left(k_{i}\right),
\end{aligned}
$$

where the fully dimensional power spectrum is given again by (2.17) and is evaluated at the discrete values $k_{i}:=(i+2)|K|^{1 / 2}$. For the flat case since the radial coordinates coincide $\chi=r$ they are fully dimensional and consequently $\nu=k$ and $S(\nu)=P(k)$ are also fully dimensional.

By using the functional forms of the $\ell=0$ scalar harmonic modes given by equations (B.23) the two-point correlation function in terms of the radial coordinate $\chi$ can be written in terms of the fully dimensional power spectrum as

$$
\xi(\chi)= \begin{cases}\int_{0}^{\infty} \frac{\mathrm{d} k k^{2}}{2 \pi^{2}} P(k) \frac{\sqrt{-K} \sin (k \chi / \sqrt{-K})}{k \sinh (\chi)} & K<0 \\ \int_{0}^{\infty} \frac{\mathrm{d} k k^{2}}{2 \pi^{2}} P(k) \frac{\sin (k \chi)}{k \chi} & K=0 \\ \sum_{i=1}^{\infty} \frac{k_{i}^{2}}{2 \pi^{2}} P\left(k_{i}\right) \frac{K \sin \left(k_{i} \chi / \sqrt{K}\right)}{k_{i} \sin (\chi)} & K>0\end{cases}
$$

where

$$
k_{i}:=(i+2) \sqrt{K} \quad \text { and } \quad P(k):= \begin{cases}\frac{S\left(|K|^{1 / 2} k\right)}{|K|^{3 / 2}} & K \neq 0 \\ S(k) & K=0 .\end{cases}
$$


One can also change the radial comoving coordinate $\chi$ to the radial comoving distance $r$ (equations (1.5)) to write

$$
\xi(r)= \begin{cases}\int_{0}^{\infty} \frac{\mathrm{d} k k^{2}}{2 \pi^{2}} P(k) \frac{\sqrt{-K} \sin (k r)}{k \sinh (\sqrt{-K} r)} & K<0, \\ \int_{0}^{\infty} \frac{\mathrm{d} k k^{2}}{2 \pi^{2}} P(k) \frac{\sin (k r)}{k r} & K=0 \\ \sum_{i=1}^{\infty} \frac{k_{i}^{2}}{2 \pi^{2}} P\left(k_{i}\right) \frac{K \sin \left(k_{i} r\right)}{k_{i} \sin (\sqrt{K} r)}=\sum_{i=1}^{\infty} \frac{k_{i} K}{2 \pi^{2}} P\left(k_{i}\right) \frac{\sin \left(k_{i} r\right)}{\sin (\sqrt{K} r)} & K>0\end{cases}
$$

Note that, independent of the radial coordinate used, equation (2.19) or (2.21), both non-flat cases converges to the flat case for scales much smaller than the curvature radius (flat-limit), $r \ll|K|^{-1 / 2}$.

\subsubsection{Estimation techniques in configuration space}

The goal of the preset paragraph is to review some basic results on methods for statistical estimations of the two-point correlation function $\xi(r)$, in order to do so, we closely follow the review presented in [36] an references therein. From astrophysical observations of $N$ objects (e.g. galaxies of some type, quasars, etc.), one can obtain information on their respective positions (angular and radial) relative to the observer inside a window $W$ of observation, defining a volume $V(W)$. This kind of information together with auxiliary astrophysical or instrumental (relative to the kind of observation) characteristics for each object defines a catalog.

Exploiting the idea that the correlation function measures the excess probability for finding a pair of objects separated by a distance $r$, different estimators are based on some kind of average of the counts of galaxy neighbors at a given scale, or more precisely, within a narrow band of scales, commonly named as a bin. A natural problem that emerges is that for galaxies close to the boundary of the window the number of neighbors is underestimated. Usually an auxiliary random sample containing $N_{R}$ points must be generated in $W$. The use of random samples in the estimators constitutes a way to implement Monte Carlo integration of the volumes [36]. This also means that the number of random points must be much larger than the size of the data sample, because this is a basic requirement of Monte Carlo estimation techniques.

\section{Properties of estimators}

As in any statistical application, concrete concepts to quantify the "goodness" of an estimator should be given. Here the definitions of bias and consistency for correlation function estimators are presented following the ideas presented in [41].

An estimator $\widehat{\xi}(r)$ is called unbiased if its expectation value $\widehat{\xi}(r)$ equals the actual value of $\xi(r)$, i.e., if

$$
\langle\widehat{\xi}(r)\rangle=\xi(r)
$$

where $\langle\cdot\rangle$ is the average over all possible realizations of the random fields whose two-point function is given by $\xi(r)$.

An estimator is called consistent if the estimates $\widehat{\xi}(r)$ obtained inside a finite sample geometry $W$ from one space filling realization, converges towards the true value of $\xi(r)$, 
as the sample volume $V(W)$ increases, i.e. if

$$
\widehat{\xi}(r) \rightarrow \xi(r)
$$

holds when $V(W) \rightarrow \infty$.

As discussed at the beginning of the present chapter, in Cosmology an ergodic-type hypothesis is commonly assumed because of the fundamental impossibility to repeat the stochastic process which generated cosmological fluctuations. If this hypothesis holds and the stochastic process underlying cosmological fluctuations is in fact ergodic, one can state that any unbiased estimator is also consistent. For this reason, we are mainly interested on the study the bias of different estimators.

\section{Pair counting-based estimators}

Astrophysical studies favor estimators based on pair counting, while most of the mathematical research is focused on geometric edge correction [42]. Here some results on pair counting-based estimators are presented.

Following [42], we begin by defining the pair counts with a function $\Phi_{r}$ symmetric on its arguments,

$$
\Phi_{r}(\mathbf{x}, \mathbf{y}):= \begin{cases}1, & r \leq d(\mathbf{x}, \mathbf{y}) \leq r+\Delta \\ 0, & \text { Otherwise }\end{cases}
$$

where $d(\mathbf{x}, \mathbf{y})$ defines a suitable separation (distance) of the two points. An important point to note here is that the distance function $d$ is cosmology-dependent for the 3D two-point correlation function, i.e. it is necessary to assume a fiducial cosmology in order to convert measured angular positions and redshifts into comoving distances. This can be an undesired property in order to perform cosmological analysis. A possible way to overcome this difficulty could be not to use directly the $3 \mathrm{D}$ correlation function but the angular two-point correlation function (see $\S 4.1 .2$ ) where the distance $d$ is the angular distance between objects, cosmology-independent in the context of FLRW models. See e.g. [43] for a recent discussion.

The pair-counts can be introduced in terms of the function $\Phi_{r}$, for example, for the case of catalog-random pairs $(D R)$ as

$$
P_{D R}(r)=\sum_{\mathbf{x} \in D} \sum_{\mathbf{y} \in R} \Phi_{r}(\mathbf{x}, \mathbf{y})
$$

where the summation runs over coordinates of points in the data set $D$ (catalog) and points in the set $R$ of randomly distributed points (random catalog), respectively. The quantity $P_{D R}(r)$ is then the number of pairs of objects, one in the true data catalog and one in the random catalog separated by distance $r . P_{D D}$ and $P_{R R}$ are defined in terms of $\Phi_{r}$ in an analogous way, with $\mathbf{x}$ and $\mathbf{y}$ taken entirely from the data and random samples, under the restriction that $\mathbf{x} \neq \mathbf{y}$.

It is convenient to introduce normalized pair counts

$$
\begin{aligned}
& D D(r)=P_{D D}(r) /(N(N-1)), \\
& D R(r)=P_{D R}(r) /\left(N N_{R}\right), \\
& R R(r)=P_{R R}(r) /\left(N_{R}\left(N_{R}-1\right)\right),
\end{aligned}
$$

where $N$ and $N_{R}$ are the total number of data and random points in the window volume. 
The simplest pair counting-based estimator implementation is the Peebles and Hauser estimator [44], first used for the study of the angular correlation function of galaxies (see $\S 4.1 .2$ ) on the Zwicky Catalog, a catalog containing 3755 galaxies covering an area on the sky of $6014.1 \mathrm{deg}^{2}$ [45]. The Pebbles and Hauser estimator $\widehat{\xi}_{\mathrm{PH}}$ is also known in the literature as the natural estimator $\widehat{\xi}_{\mathrm{N}}$ because of its straightforward interpretation as the excess probability of find pairs of galaxies separated a distance $r$ (see the discussion at the beginning of this chapter).

$$
\widehat{\xi}_{\mathrm{PH}}(r)=\widehat{\xi}_{\mathrm{N}}(r)=\frac{D D(r)}{R R(r)}-1
$$

Despite its straightforward interpretation, the natural estimator is known to suffer from insufficient correction effects related with its biasing specially at large scales where it can be of the same order of the uncertainties in the measurements [46] (see discussion of biasing of estimators in $\S 2.1 .2$ ).

A better estimator is the Davis and Peebles estimator also known in the literature as standard estimator. This estimator was introduced in [47] in the context of the analysis of the 3D two-point correlation function of galaxies on the Harvard-Smithsonian Center for Astrophysics (CfA) survey, the first wide-angle survey to reach beyond the Local Supercluster (LSC) providing strong evidence of the existence of complex structures at large scales (e.g. large voids, filaments, etc.) and consequently inspiring posterior studies on the nature of clustering. The CfA survey covered an area of 2.7 steradian with 2400 galaxies. This estimator is given by

$$
\widehat{\xi}_{\mathrm{DP}}(r)=\widehat{\xi}_{\mathrm{S}}(r)=\frac{D D(r)}{D R(r)}-1
$$

Following the chronological order on [46], a computation of the bias for the natural and standard estimators bias was presented. These computations are reviewed here in $\S 2.1 .2$ using methods slightly different from the original. [46] showed that the expectation values for the natural and standard estimators, equations (2.49) and (2.50) respectively, realizing for the first time that as long as one is dealing with volume-limited samples the biasing errors $\bar{\delta}$ and $\psi(r)$ are both nonzero (the appropriate interpretation of those errors is discussed in $\S 2.1 .2)$. These terms are of the same order and can be larger than the uncertainty in $\widehat{\xi}(r)$, specially for small correlation amplitude (at large scales), introducing a substantial bias in the estimator. In this way, Hamilton [46] came out with a first quantitative way to proceed with corrections to estimators by proposing a new estimator, the so-called Hamilton estimator,

$$
\widehat{\xi}_{\text {Ham }}(r)=\frac{D D(r) \cdot R R(r)}{D R(r)^{2}}-1,
$$

which has only second order bias, caused by the finite sample effect, equation (2.51).

The main shortcoming of the Hamilton estimator, equation (2.29), is that the $D R(r)$ term may introduce numerical noise at small distances [46, 36].

Another estimator was proposed almost simultaneously by Landy and Szalay [48], having almost the same properties, the so-called Landy-Szalay estimator:

$$
\widehat{\xi}_{\mathrm{LS}}(r):=\frac{D D(r)-2 D R(r)+R R(r)}{R R(r)}
$$




\section{The bias and the uncertainty in the mean density}

In this paragraph we show the calculations given in [46] in a simple case where the sample is volume-limited (i.e. with a constant expected density in the sample). Under this circumstances, the optimal strategy is to weight all galaxies equally. The empirical density in the catalog $n$ is a sum of Dirac delta functions over the galaxies in the catalog.

Let $\bar{n}$ to be the expected value of the density and $\delta$ the relative fluctuation in the sample:

$$
\delta(\mathbf{x})=\frac{n(\mathbf{x})-\bar{n}}{\bar{n}} .
$$

Let also $W$ denote the window function for the sample volume and $\langle\cdot\rangle$ the ensemble average, properly represented by the integration on the volume. For example, $\langle W(\mathbf{x}) n(\mathbf{x})\rangle$ represents the integration of the empirical density and thus equals the number of points in the sample,

$$
\langle W(\mathbf{x}) n(\mathbf{x})\rangle=\int \mathrm{d}^{3} \mathbf{x} W(\mathbf{x}) n(\mathbf{x})=N .
$$

It is then necessary to introduce the following quantities

$$
\begin{aligned}
\bar{\delta} & =\frac{\langle W(\mathbf{x}) \delta(\mathbf{x})\rangle}{\langle W(\mathbf{x})\rangle} \\
\psi(r) & =\frac{\langle\delta(\mathbf{x}) W(\mathbf{x}) W(\mathbf{y})\rangle_{r}}{\langle W(\mathbf{x}) W(\mathbf{y})\rangle_{r}} \\
\widehat{\xi}(r) & =\frac{\langle\delta(\mathbf{x}) \delta(\mathbf{y}) W(\mathbf{x}) W(\mathbf{y})\rangle_{r}}{\langle W(\mathbf{x}) W(\mathbf{y})\rangle_{r}}
\end{aligned}
$$

where $\bar{\delta}$ and $\psi$ are fluctuations, in the sense that they have zero expectations value, $\langle\bar{\delta}\rangle=\langle\psi\rangle=0$, and $\langle.\rangle_{r}$ defines a constrained ensemble average under the condition that the distance between pairs must be equal to $r$. It is properly represented by a double integration in the volume, restricted to $\mathbf{x}$ and $\mathbf{y}$ separated by a distance in $r$. For example,

$$
\langle\delta(\mathbf{x}) W(\mathbf{x}) W(\mathbf{y})\rangle_{r}=\iint_{d(\mathbf{x}, \mathbf{y})=r} \mathrm{~d}^{3} \mathbf{x} \mathrm{d}^{3} \mathbf{y} \delta(\mathbf{x}) W(\mathbf{x}) W(\mathbf{y}) .
$$

The definition of $\widehat{\xi}$ in equation (2.35) allows for its interpretation as the unbiased estimator of the real $\xi$. The important point is that it is not possible to calculate this quantity as long as the value of $\bar{n}$, i.e., the mean density, is unknown. The $\bar{\delta}$ quantity, equation (2.33), represents the mean of the fluctuation field over the volume and $\psi$, equation (2.34), can be interpreted as an unbiased estimator for the cross-correlation of the fluctuation field and a homogeneous (random) field also on the volume considered.

In order to introduce, and relate, the pair counts with the previous formalism, it is useful to introduce the abbreviated notation used by [46]. The pair counting of a general catalog can be considered as an ensemble average over pairs on a given scale,

$$
D D(r)=\langle N N\rangle /\left(\bar{n}^{\mathrm{est}}\right)^{2}
$$

where

$$
\bar{n}^{\mathrm{est}}=\frac{\langle N\rangle}{\langle W\rangle},
$$

defines the catalog-estimated galaxy number density. 
In the last expressions, $N$ denotes real galaxies weighted by some weight function $w$, and $W$ denotes the catalog window function, which is the catalog selection function $\Phi$ weighted by the same weight function $w$ of the galaxies.

The angular brackets in terms related to point counts like $\langle N\rangle$ denote averages over points in the catalog whereas for terms that imply pair counts like $\langle N N\rangle$, the average is over pairs in some infinitesimal interval of separations, say $[r, r+\Delta]$.

In general, one needs to introduce the pair weighting field $w(\mathbf{x}, \mathbf{y})$ applied to $\langle N N\rangle$ and $\langle N W\rangle$ unrelated to the point weighting field $w(\mathbf{x})$ applied to $\langle N\rangle$ and $\langle W\rangle$. Indeed, the pair weighting field need not be separable and may be different for different pair separations. It is useful therefore to define the pair window $W(\mathbf{x}, \mathbf{y})$ as the pair-weighted product of selection functions,

$$
W(\mathbf{x}, \mathbf{y}):=w(\mathbf{x}, \mathbf{y}) \Phi(\mathbf{x}) \Phi(\mathbf{y})
$$

which should be distinguished from the point window, defined as the point-weighted selection function,

$$
W(\mathbf{x}):=w(\mathbf{x}) \Phi(\mathbf{x}) .
$$

Let $n(\mathbf{x})$ denote the true galaxy density field, the underling population from which the observed galaxies are drawn. The observed galaxy density field $N^{\text {obs }}(\mathbf{x})$ should then be written as the true galaxy density weighted by the selection function

$$
N^{\mathrm{obs}}(\mathbf{x})=\Phi(\mathbf{x}) n(\mathbf{x}) .
$$

We can now introduce pair density fields

$$
\begin{aligned}
N N(\mathbf{x}, \mathbf{y}) & =w(\mathbf{x}, \mathbf{y}) N^{\mathrm{obs}}(\mathbf{x}) N^{\mathrm{obs}}(\mathbf{y})=w(\mathbf{x}, \mathbf{y}) \Phi(\mathbf{x}) \Phi(\mathbf{y}) n(\mathbf{x}) n(\mathbf{y}) \\
& =W(\mathbf{x}, \mathbf{y}) n(\mathbf{x}) n(\mathbf{y}) \\
N W(\mathbf{x}, \mathbf{y}) & =w(\mathbf{x}, \mathbf{y}) N^{\mathrm{obs}}(\mathbf{x}) \Phi(\mathbf{y})=w(\mathbf{x}, \mathbf{y}) \Phi(\mathbf{x}) n(\mathbf{x}) \Phi(\mathbf{y}) \\
& =W(\mathbf{x}, \mathbf{y}) n(\mathbf{x}) \\
W W(\mathbf{x}, \mathbf{y}) & =w(\mathbf{x}, \mathbf{y}) \Phi(\mathbf{x}) \Phi(\mathbf{y})=: W(\mathbf{x}, \mathbf{y})
\end{aligned}
$$

and also the point density field

$$
N(\mathbf{x})=w(\mathbf{x}) N^{\mathrm{obs}}(\mathbf{x})=w(\mathbf{x}) \Phi(\mathbf{x}) n(\mathbf{x})=W(\mathbf{x}) n(\mathbf{x}) .
$$

In terms of these density fields, the pair counts, involved in the different estimators for the two-point correlation function, can be interpreted as

$$
\begin{aligned}
& D D(r)=\frac{\langle N N\rangle}{\left(\bar{n}^{\mathrm{est}}\right)^{2}}=\frac{\langle W(\mathbf{x}, \mathbf{y}) n(\mathbf{x}) n(\mathbf{y})\rangle_{r} \cdot\langle W(\mathbf{x})\rangle^{2}}{\langle W(\mathbf{x}) n(\mathbf{x})\rangle^{2}} \\
& D R(r)=\frac{\langle N W\rangle}{\bar{n}^{\mathrm{est}}}=\frac{\langle W(\mathbf{x}, \mathbf{y}) n(\mathbf{x})\rangle_{r} \cdot\langle W(\mathbf{x})\rangle}{\langle W(\mathbf{x}) n(\mathbf{x})\rangle} \\
& R R(r)=\langle W W\rangle=\langle W(\mathbf{x}, \mathbf{y})\rangle_{r} .
\end{aligned}
$$

It is important to remember that the average $\langle\cdot\rangle_{r}$ is formally defined as a double integral (in $\mathbf{x}$ and $\mathbf{y}$ ) over the region defined by the restriction $d(\mathbf{x}, \mathbf{y})=r$ (see e.g. equation (2.36)).

At this point, all the ingredients necessary to perform the computation of the expected value of any of the estimators for the two-point correlation function based on pair counts 
discussed on the previous paragraph have been defined. For example, for the PH (Natural) estimator one can begin by combining the definition (2.27) with equations (2.46)-(2.48),

$$
\begin{aligned}
\widehat{\xi}_{\mathrm{N}}(r) & =\frac{D D(r)}{R R(r)}-1 \\
& =\frac{\langle W(\mathbf{x}, \mathbf{y}) n(\mathbf{x}) n(\mathbf{y})\rangle_{r}\langle W(\mathbf{x})\rangle^{2}}{\langle W(\mathbf{x}) n(\mathbf{x})\rangle^{2}\langle W(\mathbf{x}, \mathbf{y})\rangle_{r}}-1
\end{aligned}
$$

then introducing the overdensity (fluctuation) field via equation (2.31), i.e. by writing $n(\mathbf{x})=\bar{n}[\delta(\mathbf{x})+1]$, it follows that

$$
\begin{aligned}
\left\langle\widehat{\xi}_{\mathrm{N}}(r)\right\rangle= & \frac{\langle W(\mathbf{x}, \mathbf{y})[\delta(\mathbf{x})+1][\delta(\mathbf{y})+1]\rangle_{r}\langle W(\mathbf{x})\rangle^{2}}{\langle W(\mathbf{x})[\delta(\mathbf{x})+1]\rangle^{2}\langle W(\mathbf{x}, \mathbf{y})\rangle_{r}}-1 \\
= & \frac{\left[\langle W(\mathbf{x}, \mathbf{y}) \delta(\mathbf{x}) \delta(\mathbf{y})\rangle_{r}+\langle W(\mathbf{x}, \mathbf{y}) \delta(\mathbf{x})\rangle_{r}+\langle W(\mathbf{x}, \mathbf{y}) \delta(\mathbf{y})\rangle_{r}+\langle W(\mathbf{x}, \mathbf{y})\rangle_{r}\right]\langle W(\mathbf{x})\rangle^{2}}{[\langle W(\mathbf{x}) \delta(\mathbf{x})\rangle+\langle W(\mathbf{x})\rangle]^{2}\langle W(\mathbf{x}, \mathbf{y})\rangle_{r}}-1 \\
= & \frac{\langle W(\mathbf{x}, \mathbf{y}) \delta(\mathbf{x}) \delta(\mathbf{y})\rangle_{r}+\langle W(\mathbf{x}, \mathbf{y}) \delta(\mathbf{x})\rangle_{r}+\langle W(\mathbf{x}, \mathbf{y}) \delta(\mathbf{y})\rangle_{r}+\langle W(\mathbf{x}, \mathbf{y})\rangle_{r}}{\langle W(\mathbf{x}, \mathbf{y})\rangle_{r}} \\
& \times \frac{\langle W(\mathbf{x})\rangle^{2}}{\langle W(\mathbf{x}) \delta(\mathbf{x})\rangle^{2}+\langle W(\mathbf{x})\rangle^{2}+2\langle W(\mathbf{x}) \delta(\mathbf{x})\rangle\langle W(\mathbf{x})\rangle}-1 \\
= & {\left[\frac{\langle W(\mathbf{x}, \mathbf{y}) \delta(\mathbf{x}) \delta(\mathbf{y})\rangle_{r}}{\langle W(\mathbf{x}, \mathbf{y})\rangle_{r}}+2 \frac{\langle W(\mathbf{x}, \mathbf{y}) \delta(\mathbf{x})\rangle_{r}}{\langle W(\mathbf{x}, \mathbf{y})\rangle_{r}}+1\right] } \\
& \times\left[\frac{\langle W(\mathbf{x}) \delta(\mathbf{x})\rangle^{2}}{\langle W(\mathbf{x})\rangle^{2}}+2 \frac{\langle W(\mathbf{x}) \delta(\mathbf{x})\rangle}{\langle W(\mathbf{x})\rangle}+1\right]^{-1}-1 \\
= & \frac{\widehat{\xi}(r)+2 \psi(r)+1}{(\bar{\delta}+1)^{2}}-1 \\
\left\langle\widehat{\xi}_{\mathrm{N}}(r)\right\rangle= & \frac{\widehat{\xi}(r)+2[\psi(r)-\bar{\delta}]-\bar{\delta}^{2}}{(\bar{\delta}+1)^{2}}
\end{aligned}
$$

where in the last couple of equalities, definitions (2.33)-(2.35) were used.

Computations with the other estimators are completely analogous and one can show that

$$
\begin{aligned}
\left\langle\widehat{\xi}_{\mathrm{N}}(r)\right\rangle & =\frac{\widehat{\xi}(r)+2 \psi(r)-2 \bar{\delta}-\bar{\delta}^{2}}{[1+\bar{\delta}]^{2}} \\
\left\langle\widehat{\xi}_{\mathrm{S}}(r)\right\rangle & =\frac{\widehat{\xi}(r)+\psi(r)-\bar{\delta}-\psi(r) \bar{\delta}}{[1+\bar{\delta}][1+\psi(r)]} \\
\left\langle\widehat{\xi}_{\mathrm{Ham}}(r)\right\rangle & =\frac{\widehat{\xi}(r)-\psi(r)^{2}}{[1+\psi(r)]^{2}} \\
\left\langle\widehat{\xi}_{\mathrm{LS}}(r)\right\rangle & =\frac{\widehat{\xi}(r)-2 \bar{\delta} \psi(r)+\bar{\delta}^{2}}{[1+\bar{\delta}]^{2}} .
\end{aligned}
$$

These formulae explain the superiority of Hamilton and Landy-Szalay estimators, with $\psi$ and $\bar{\delta}$ terms at the second order in the numerator. Terms in the denominator are not important since they generate a small relative error, whereas terms in the numerator can generate a high relative error when their values become non-negligible compared to $\widehat{\xi}$, i.e. a significant bias for the estimator. For Hamilton and Landy-Szalay estimators, the error 
is dominated by that of $\widehat{\xi}$ and not really affected by $\psi$ and $\bar{\delta}$, which are linked to the uncertainty in $\bar{n}$.

With these formulae it can also be shown that the estimators are biased in the general case. Indeed $\bar{\delta}$ and $\psi(r)$ have null expectation value and $\widehat{\xi}(r)$ has expected value $\xi(r)$, but the terms are combined in multiplications and divisions. So we do not get the expected value of the left-hand side by replacing each term by its expected value in the right-hand side of equations (2.49), (2.50), (2.51), (2.52).

\subsubsection{Comparison of different estimators}

Several attempts have been made to compare different estimators, using real data samples, $N$-body simulations and point processes with known correlation functions, see e.g. [49, 42]. The results are basically the same, the differences between estimators provides indications of the best estimators to use to be Hamilton and Landy-Szalay. However, an important remark pointed by [42] is that Landy-Szalay is easier to implement than Hamilton, a highly desired property. Anyway, a good way to proceed on specific applications is to compare the performance of all the estimators for the specific catalog considered. Note that in this work we do not considered the variance of the estimators. A discussion of this important statistical quantity can be found e.g. on [48].

\subsection{Harmonic space}

In this section some results on the two-point correlation function of random fields on the unit sphere $\mathbb{S}_{2}$ in harmonic space are presented. Random fields coming from radial projections of 3D fields are considered. We begin by defining the angular power spectrum and consider its relation to the power spectrum of the 3D (unprojected) field, this is done following the treatment of section II of [50], but extending its ideas to the context of non spatially flat backgrounds. We also find explicit relations between these quantities depending on the sign of the spatial curvature considered. The section ends with the review of a naive estimation technique for the angular power spectrum from data on the entire unit sphere.

\subsubsection{Angular power spectrum}

Consider a general field, $s(\chi, \theta, \varphi)$, under the assumptions of statistical homogeneity and isotropy in the Universe. Suppose further that, from this three-dimensional field, a set of two-dimensional scalar fields $\left\{x^{i}(\mathbf{n})\right\}$ (where $\mathbf{n}$ represents a direction on the sky) is constructed via appropriated weighted projections,

$$
x^{i}(\mathbf{n})=x^{i}(\theta, \varphi)=\int_{0}^{\infty} \mathrm{d} z W^{x^{i}}(z) s(\chi(z), \theta, \varphi),
$$

where $W^{x^{i}}(z)$ are suitable window functions in redshift for the fields $\left\{x^{i}\right\}$.

Our goal is to deduce a general form of the angular auto- and cross- power spectra of the fields $x^{i}$ in terms of that of the field $s$. As the fields are properly defined on the sky, let us expand them in Spherical Harmonics on the unit sphere,

$$
x^{i}(\mathbf{n})=\sum_{\ell=0}^{\infty} \sum_{m=-\ell}^{\ell} x_{\ell m}^{i} Y_{\ell}^{m}(\mathbf{n}),
$$


and, according to the statistical isotropy assumption, to define the angular power spectrum between two fields $x^{i}$ and $x^{j}$ by the expectation value

$$
\left\langle x_{\ell m}^{i} x_{\ell^{\prime} m^{\prime}}^{j *}\right\rangle=\delta_{\ell \ell^{\prime}} \delta_{m m^{\prime}} C_{\ell}^{x^{i} x^{j}}
$$

Thus, as a simple consequence of the addition theorem of Spherical Harmonics [37],

$$
P_{\ell}\left(\mathbf{n}_{1} \cdot \mathbf{n}_{2}\right)=\frac{4 \pi}{2 \ell+1} \sum_{m=-\ell}^{\ell} Y_{\ell}^{m *}\left(\mathbf{n}_{1}\right) Y_{\ell}^{m}\left(\mathbf{n}_{2}\right),
$$

the two-point correlation function (in configuration space) between the fields $x^{i}$ and $x^{j}$ can be obtained in terms of the angular power spectrum as an appropriate Legendre transform:

$$
\left\langle x^{i}(\mathbf{n}) x^{j *}\left(\mathbf{n}^{\prime}\right)\right\rangle=\sum_{\ell=0}^{\infty} \frac{2 \ell+1}{4 \pi} C_{\ell}^{x^{i} x^{j}} P_{\ell}\left(\mathbf{n} \cdot \mathbf{n}^{\prime}\right) .
$$

On the other hand, we can expand the three-dimensional field $s$ into harmonic modes as

$$
s(\chi, \theta, \varphi)= \begin{cases}\sum_{\ell m} \int_{0}^{\infty} \frac{\mathrm{d} \nu \nu^{2}}{2 \pi^{2}} s_{\ell m}(\nu) \widehat{X}_{\ell}^{(-, 0)}(\chi, \nu) Y_{\ell}^{m}(\theta, \varphi) & K \leq 0, \\ \sum_{\ell m} \sum_{\nu=3}^{\infty} \frac{\nu^{2}}{2 \pi^{2}} s_{\ell m}(\nu) \widehat{X}_{\ell}^{(+)}(\chi, \nu) Y_{\ell}^{m}(\theta, \phi) & K>0,\end{cases}
$$

where the $\widehat{X}_{\ell}$ functions are the correctly normalized radial modes on the constant time hypersurfaces $\Sigma_{t}$ of FLRW Universe models, see appendix B. Inserting these expansions on the projection relation between the $x^{i}$ and $s$ fields we have the following expansions for the projected fields:

$$
x^{i}(\theta, \varphi)= \begin{cases}\int_{0}^{\infty} \mathrm{d} z W^{x^{i}}(z) \sum_{\ell m} \int_{0}^{\infty} \frac{\mathrm{d} \nu \nu^{2}}{2 \pi^{2}} s_{\ell m}(\nu) \widehat{X}_{\ell}^{(-, 0)}(\chi, \nu) Y_{\ell}^{m}(\theta, \varphi) & K \leq 0, \\ \int_{0}^{\infty} \mathrm{d} z W^{x^{i}}(z) \sum_{\ell m} \sum_{\nu=3}^{\infty} \frac{\nu^{2}}{2 \pi^{2}} s_{\ell m}(\nu) \widehat{X}_{\ell}^{(+)}(\chi, \nu) Y_{\ell}^{m}(\theta, \varphi) & K>0 .\end{cases}
$$

Taking the two-point correlation of arbitrary fields, $x^{i}$ and $x^{j}$, we have

$$
\begin{aligned}
\left\langle x^{i}(\mathbf{n}) x^{j *}\left(\mathbf{n}^{\prime}\right)\right\rangle= & \int_{0}^{\infty} \mathrm{d} z W^{x^{i}}(z) \int_{0}^{\infty} \mathrm{d} z^{\prime} W^{x^{j}}\left(z^{\prime}\right) \\
& \times \sum_{\ell m} \sum_{\ell^{\prime} m^{\prime}} \int_{0}^{\infty} \frac{\mathrm{d} \nu \nu^{2}}{2 \pi^{2}} \int_{0}^{\infty} \frac{\mathrm{d} \nu^{\prime} \nu^{\prime 2}}{2 \pi^{2}} \widehat{X}_{\ell}^{(-, 0)}(\chi, \nu) \widehat{X}_{\ell^{\prime}}^{(-, 0)}\left(\chi^{\prime}, \nu^{\prime}\right) \\
& \times Y_{\ell}^{m}(\mathbf{n}) Y_{\ell^{\prime}}^{m^{\prime} *}\left(\mathbf{n}^{\prime}\right)\left\langle s_{\ell m}(\nu) s_{\ell^{\prime} m^{\prime}}^{*}\left(\nu^{\prime}\right)\right\rangle
\end{aligned}
$$

for the spatially open and flat cases $(K \leq 0)$, and analogously

$$
\begin{aligned}
\left\langle x^{i}(\mathbf{n}) x^{j *}\left(\mathbf{n}^{\prime}\right)\right\rangle= & \int_{0}^{\infty} \mathrm{d} z W^{x^{i}}(z) \int_{0}^{\infty} \mathrm{d} z^{\prime} W^{x^{j}}\left(z^{\prime}\right) \\
& \times \sum_{\ell m} \sum_{\ell^{\prime} m^{\prime}} \sum_{\nu=3}^{\infty} \frac{\nu^{2}}{2 \pi^{2}} \sum_{\nu^{\prime}=3}^{\infty} \frac{\nu^{\prime 2}}{2 \pi^{2}} \widehat{X}_{\ell}^{(+)}(\chi, \nu) \widehat{X}_{\ell^{\prime}}^{(+)}\left(\chi^{\prime}, \nu^{\prime}\right) \\
& \times Y_{\ell}^{m}(\mathbf{n}) Y_{\ell^{\prime}}^{m^{\prime} *}\left(\mathbf{n}^{\prime}\right)\left\langle s_{\ell m}(\nu) s_{\ell^{\prime} m^{\prime}}^{*}\left(\nu^{\prime}\right)\right\rangle,
\end{aligned}
$$


for the spatially closed case $(K>0)$.

Next, we introduce the 3D power spectrum of the field $s, S^{s}$. According to the assumptions of statistical homogeneity and isotropy for $s^{1}$,

$$
\left\langle s_{\ell m}(\nu) s_{\ell^{\prime} m^{\prime}}^{*}\left(\nu^{\prime}\right)\right\rangle= \begin{cases}(2 \pi)^{3} \delta_{\ell \ell^{\prime}} \delta_{m m^{\prime}} \frac{\delta_{D}^{(1)}\left(\nu-\nu^{\prime}\right)}{\nu^{2}} S^{s}(\nu) & K \leq 0, \\ (2 \pi)^{3} \delta_{\ell \ell^{\prime}} \delta_{m m^{\prime}} \frac{\delta_{\nu \nu^{\prime}}}{\nu^{2}} S^{s}(\nu) & K>0,\end{cases}
$$

so that, for example, for the spatially open and flat cases we can simplify our result to

$$
\begin{aligned}
\left\langle x^{i}(\mathbf{n}) x^{j *}\left(\mathbf{n}^{\prime}\right)\right\rangle= & \int_{0}^{\infty} \mathrm{d} z W^{x^{i}}(z) \int_{0}^{\infty} \mathrm{d} z^{\prime} W^{x^{j}}\left(z^{\prime}\right) \\
& \times \frac{2}{\pi} \int_{0}^{\infty} \mathrm{d} \nu \nu^{2} \sum_{\ell} \widehat{X}_{\ell}^{(-, 0)}(\chi, \nu) \widehat{X}_{\ell}^{(-, 0)}\left(\chi^{\prime}, \nu\right) \\
& \times \sum_{m} Y_{\ell}^{m}(\mathbf{n}) Y_{\ell}^{m *}\left(\mathbf{n}^{\prime}\right) S^{s}(\nu) .
\end{aligned}
$$

Using again the addition theorem of Spherical Harmonics, equation (2.56), we get

$$
\begin{aligned}
\left\langle x^{i}(\mathbf{n}) x^{j *}\left(\mathbf{n}^{\prime}\right)\right\rangle= & \sum_{\ell} \frac{2 \ell+1}{4 \pi} P_{\ell}\left(\mathbf{n} \cdot \mathbf{n}^{\prime}\right) \int_{0}^{\infty} \mathrm{d} z W^{x^{i}}(z) \int_{0}^{\infty} \mathrm{d} z^{\prime} W^{x^{j}}\left(z^{\prime}\right) \\
& \times \frac{2}{\pi} \int_{0}^{\infty} \mathrm{d} \nu \nu^{2} \widehat{X}_{\ell}^{(-, 0)}(\chi, \nu) \widehat{X}_{\ell}^{(-, 0)}\left(\chi^{\prime}, \nu\right) S^{s}(\nu)
\end{aligned}
$$

therefore, combining equations (2.57) and (2.63) we finally can arrive to

$$
\begin{aligned}
C_{\ell}^{x^{i} x^{j}}= & \int_{0}^{\infty} \mathrm{d} z W^{x^{i}}(z) \int_{0}^{\infty} \mathrm{d} z^{\prime} W^{x^{j}}\left(z^{\prime}\right) \\
& \times\left[\frac{2}{\pi} \int_{0}^{\infty} \mathrm{d} \nu \nu^{2} S^{s}(\nu) \widehat{X}_{\ell}^{(-, 0)}(\chi, \nu) \widehat{X}_{\ell}^{(-, 0)}\left(\chi^{\prime}, \nu\right)\right] .
\end{aligned}
$$

The analogous result for a spatially closed Universe model is given by

$$
C_{\ell}^{x^{i} x^{j}}=\int_{0}^{\infty} \mathrm{d} z W^{x^{i}}(z) \int_{0}^{\infty} \mathrm{d} z^{\prime} W^{x^{j}}\left(z^{\prime}\right)\left[\frac{2}{\pi} \sum_{\nu=3}^{\infty} \nu^{2} S^{s}(\nu) \widehat{X}_{\ell}^{(+)}(\chi, \nu) \widehat{X}_{\ell}^{(+)}\left(\chi^{\prime}, \nu\right)\right] .
$$

An important remark here is to note that the radial coordinate distance inside the radial modes $\chi$ represents the radial distance-redshift relation in the Universe $\chi=\chi(z)$, equation (1.19).

As final remark we can see that from the expresion for the APS for spatially open and flat Universe models, equation (2.65), one easily recover the standar expression for the spatially flat Universe which commonly appears in the liteature. By replacing the form of the radial haarmonics in terms of the spherical Bessel functions $\widehat{X}_{\ell}^{(0)}(\chi, \nu)=(-1)^{\ell} j_{\ell}(\chi \nu)$, see equations (B.27), easily follows

$$
C_{\ell}^{x^{i} x^{j}}=\int_{0}^{\infty} \mathrm{d} z W^{x^{i}}(z) \int_{0}^{\infty} \mathrm{d} z^{\prime} W^{x^{j}}\left(z^{\prime}\right)\left[\frac{2}{\pi} \int_{0}^{\infty} \mathrm{d} \nu \nu^{2} S^{s}(\nu) j_{\ell}(\chi \nu) j_{\ell}\left(\chi^{\prime} \nu\right)\right] .
$$

\footnotetext{
${ }^{1}$ It is important to note that for cases where the field $s$ is not statistically homogeneous and isotropic this expansion is no longer valid, for example, when $s$ is identified with the field of total matter fluctuations in redshift space.
} 


\subsubsection{Simple estimator for the angular power spectra}

In this paragraph we present some basic preliminary work on measurement techniques for the angular power spectrum of a field defined on the unit sphere $\mathbb{S}_{2}$ representing the observer sky. We closely follow the basic treatment presented on [51].

Begin by consider a measurement of a real field $x(\mathbf{n})$ on $\mathbb{S}_{2}$. Here $x$ represent an arbitrary cosmological observable, for example, the projected fluctuation of the galaxy density $\delta_{\mathrm{g}}^{2 \mathrm{D}}$ or the convergence field of galaxies on the foreground generated by matter in between to the observer $\kappa$, see section 1.4. This measurement can be described in terms of its spherical harmonic coefficients $\left\{x_{\ell m}\right\}$ (equation (2.70)) in terms of which, an observed angular power spectrum $C_{\ell}$ of $x$ is given by the inversion of equation (2.70) [4],

$$
C_{\ell}=\sum_{m=-\ell}^{\ell} \frac{x_{\ell m} x_{\ell m}^{*}}{2 \ell+1} .
$$

If $x$ is an isotropic Gaussian random field, the power spectrum contain all the statistical information of the field. Moreover, the observed power spectrum $C_{\ell}$ should be considered as a realization of a theoretical power spectrum $C_{\ell}^{\text {theo }}$ that, again, fully characterizes the field of interest $x$. The variance of the theoretical power spectrum, the so-called in Cosmology cosmic variance, depends on the number of modes on the sky, as stated at the beginning of the present chapter it is related with the scales allowed by observations, i.e., by the size of the observable Universe, and is given by (see e.g. chapter 11 of [4])

$$
\operatorname{Var}\left(C_{\ell}\right)=\frac{2\left(C_{\ell}^{\text {theo }}\right)^{2}}{2 \ell+1}
$$

In real applications, the data should contain a finite amount of information because the continuous signal of $x$ is observed at finite resolution on the sky and also because of other observational issues. Consequently, this subtleties should be taken into account in the estimation of the $C_{\ell}$. However, in the present work we are only presenting a first approximation to this problem, so we will not cover this issues. For a recent discussion see e.g. [51].

In the context of Large surveys of galaxies, data catalogs usually provide lists of objects whose positions and other astrophysical properties, such as photometric colors, were measured in the context of the survey, containing information of the 3D distribution of objects and its properties. Then the main task is to relate the properties in such lists to the specific fields defined on the sphere that can be modeled inside cosmological models. In order to do so, a catalog must be translated into pixelized map(s) on the sphere which are observational discrete representations of the fields. For example, following [51] if we consider the field of fluctuations in the number of galaxies in the context of a given survey, the catalog produced by the survey should be converted into into a number count pixelized map $\tilde{\mathbf{n}}$, and then transformed into an overdensity map $\tilde{\delta}$, i.e., the actual map representing the observation of the $\delta_{\mathrm{g}}^{2 \mathrm{D}}$ field. Given a pixelization scheme, if $n_{p}$ denotes the number of objects in the $p$-th pixel, which can be thought to be generically described by its size $\Omega_{p}$ and the position of its center $\mathbf{n}_{p}$, the pixelized overdensities can be constructed as

$$
\widehat{\delta}_{p}=\frac{\widehat{n}_{p}}{\Omega_{p} \bar{n}}-1
$$

where $\bar{n}=\mathrm{N}_{\text {obj }} / \Delta \Omega$ is the average number of objects per steradian and $\mathrm{N}_{\text {obj }}$ is the total number of objects in the catalog. 
The following task is to use the pixelized maps to properly estimate the angular power spectrum of the underlying field. The initial natural problem arising on the design of a statistical estimator for (2.67) is the fact that with a pixelized map it is impossible to reproduce a full harmonic decomposition, i.e., it is impossible to construct the $x_{\ell m}$ coefficients for all the values of $\ell$ and $m$. One always has to begin the estimation from a band-limited representation of the field associated with the pixelized map.

There are different alternatives (conventions) to represent pixelized maps and their harmonic decomposition. In this work the HEALPix convention will be adopted. Originally developed to address the data processing and analysis needs of cosmic microwave background (CMB) experiments, HEALPix ${ }^{2}$, acronym for the Hierarchical Equal Area isoLatitude Pixelization, is specially designed to manage very large volumes of astronomical data and large area surveys in the form of discretized spherical maps [52].

In HEALPix conventions, a band-limited field $x$ defined on the unit sphere $\mathbb{S}_{2}$ is decomposed in Spherical Harmonics as

$$
x(\mathbf{n})=\sum_{\ell=0}^{\ell_{\max }} \sum_{m=-\ell}^{\ell} x_{\ell m} Y_{\ell}^{m}(\mathbf{n}),
$$

where it is assumed that there is insignificant signal power in modes with $\ell>\ell_{\max }$. As was discussed, pixelizing $x(\mathbf{n})$ can be understood as sampling it at $N_{\text {pix }}$ locations $\mathbf{n}_{p}$, $p \in\left[0, N_{\text {pix }}\right]$. The sample function values $x_{p}$ can then be used to estimate the spherical harmonic decomposition of the field $x_{\ell m}$. The most basic estimator that can be conceived is given by

$$
\widehat{x}_{\ell m}=\frac{4 \pi}{N_{\mathrm{pix}}} \sum_{p=0}^{N_{\mathrm{pix}}-1} Y_{\ell}^{m *}\left(\mathbf{n}_{p}\right) x_{p} .
$$

It is implemented as part of the library of the HEALPix code in the ANAFAST routine along with other more sophisticated routines for estimation of the spherical harmonic decomposition. This routine is specially designed to perform harmonic analysis of HEALPix maps up to a maximum spherical harmonic order $\ell_{\max }$. The principal advantage of the iso-latitude and equal area pixelization in HEALPix is the computation speed of the $x_{\ell m}$, given a pixelized map with $N_{\text {pix }}$ pixels, the total computation scales as $\mathcal{O}\left(N_{\text {pix }}^{3 / 2}\right)$ with a prefactor depending on $\ell_{\max }$ in contrast to non iso-latitude schemes of pixelization of the sphere, for which the same computation commonly scales as $\mathcal{O}\left(N_{\text {pix }}^{2}\right)$ [52].

Taking into account this computational advantage, in this work the ANAFAST routine is used to compute the estimator for the harmonic modes given in equation (2.71) and then an estimator for the first $\ell_{\max } C_{\ell}$ 's is constructed from its definition, equation (2.67).

Note that such an estimator is a first approximation to the real estimation problem in cosmology. The main drawback of this estimator is not taken into account the intrinsic shot noise present on any cosmological signal, and then comes another issues like, for example, masking effects and corrections for observational systematics. Indeed, for regimes in which the shot noise contribution can be negligible and all the real data problems are not present, like, for example, a simulated all-sky map of a cosmological field this estimator can be still used as a first approximation. In this work we use the estimation technique just depicted to measure the angular power spectrum of dark matter fluctuations on a $N$-body cosmological simulation, namely, the Onion simulation [13], as well as the auto-correlation of the weak lensing convergence field and its cross-correlation with

\footnotetext{
${ }^{2}$ http://healpix.jpl.nasa.gov/
} 
dark matter fluctuations, showing the points stated above. Our results are presented on $\S 4.2$. 


\section{Chapter 3}

\section{Statistical inference}

In the recent years cosmology has entered into its "golden age" as long as specially designed experiments has allowed the field of precision cosmology to advance. This developments have brought the problem of how to properly extract conclusions about our cosmological models from contrasting them with observations. In order to solve this problem cosmology uses the methods of statistical inference, the main theme of the present chapter.

Statistical inference is a branch of statistics devoted to draw conclusions from data subject to random variation, for example, observational and/or experimental errors present on physical systems or sampling variation. At least two different schools of statistical inference can be distinguished: first, the most common one (the "orthodox" if you want) the frequentist statistical inference, where the probability of an event is determined by the ratio of the number of times $M$ the event occurred in $N$ experiments, $p=M / N$, when $N \rightarrow \infty$. It is important to note that this interpretation requires the experiment to be repeatable. Thus, Frequentist inference is objective, in the sense that probability is not the property of a particular event, but the property of the ensemble or parent population. In second place there is the Bayesian statistical inference which on an over-simplified view, basically describes "degrees of belief" using probability theory. Bayesian inference is subjective, in the sense that it allows to assign probability to predictions or theories (models), supported by the available evidence.

It is important to note that the difference between the above schools of statistical inference is inherently related to the interpretation of the notion of probability [53, 54].

In the present chapter the basics of Bayesian inference as a tool to use observations to test cosmological models and update the "degree of belief" on them using available data is presented. We closely follow the treatment of [54]. The problem of sampling from a probability distribution function (PDF) is depicted in the context of Bayesian parameter inference and the most widely used method of Monte Carlo Markov chain (MCMC) sampling in Cosmology is reviewed. Sampling methods based on Markov chains were first developed for applications in statistical physics. The paper of Metropolis [55] introduce the concept nowadays known as the Metropolis algorithm in which the next state in a Markov chain is chosen by considering a (usually small) change to the actual state, and accepting or rejecting this change, based on how the probability of the altered state compared to that of the current state, see e.g., Chapter 16 of [56]. The recent alternative method of affine-invariant MCMC [57] is also discussed as a useful alternative for solving efficiently the problems of sampling degenerate PDFs with the possibility of using parallel computational resources. 


\subsection{Bayes' Theorem}

Bayes' Theorem can be understood as a "simple" consequence of the basic rules (axioms) of probability theory. However, with amazing implications, giving the rules by which probabilities (understood as degree of belief in propositions) should be interpreted and manipulated. Here we present a description of the Bayes' theorem by closely follow the treatment of [54], showing that the basic rules of dealing with probabilities follow from basic plausible properties of probability distributions.

Consider a proposition $A$ and its negation $\neg A$, then its associated probabilities should follow a sum rule in the sense that, $p(A \mid I)+p(\neg A \mid I)=1$, where the vertical bar means that the probability assignment is conditional, i.e., it is properly assuming whatever information is given on its right. This sum rule tells nothing but that for $A$ one of the propositions should be true, itself or its negation. This sum rule can then be extended to more general grounds by considering $A$ to be an arbitrary event, e.g. a random variable or, more related to the present work, the value of an specific parameter inside some mathematical model (e.g. the content of Dark Matter in the Universe on the context of the standard model of Cosmology). The natural generalization of the sum rule should be of the form

$$
\sum_{A} p(A \mid I)=1
$$

where the sum runs over the possible outcomes for $A$ and, again, $I$ represents any relevant information that is assumed to be true. Note that this sum rule states the known fact that probability distributions should be normalized to the unity.

A second basic requirement for probabilities is a product rule,

$$
p(A, B \mid I)=p(A \mid B, I) p(B \mid I)
$$

stating that the joint probability of events $A$ and $B$ (left-hand side) should be equal to the probability of $A$ given that $B$ occurs times the probability of $B$ occurring on its own, all conditional on information $I$. Note that this statement can be also understood as a generalization of the definition of the notion of joint probability when additional information $I$ is given. In fact, when no information $I$ is taken into account on (3.2) it is equivalent to the standard definition of conditional probability, $p(A \mid B)=p(A, B) / p(B)$.

In principle one can deduce notably interesting properties of the probability distributions from only this two basic requirements. Of fundamental importance for inference process, one can mention, the notion of marginalization and, the extremely important Bayes' theorem.

Let us begin with the notion of marginalization. Suppose one is interested in the probability of $B$ alone, irrespective of $A$, then, the sum and product rules together imply that

$$
p(B \mid I)=\sum_{A} p(A, B \mid I),
$$

where the sum runs over the possible outcomes for proposition (event) $A$. The result follows simply by summing over all possible outcomes for $A$ on both sides of the product rule (3.2) and then use the sum rule on the left-hand-side of the result. The quantity on the left-hand-side of (3.3) defines what is called marginal probability of $B$ coming from the joint probability of $A$ and $B p(A, B)$. 
Now we can turn to the Bayes' theorem. It simply follows from realize that as long as $p(A, B \mid I)=p(B, A \mid I)$, the product rule can be rewritten as

$$
p(B \mid A, I)=\frac{p(A \mid B, I) p(B \mid I)}{p(A \mid I)} .
$$

In physics or science in general, the interpretation and usefulness of the Bayes' theorem, equation (3.4), is more clear when one replaces general event $A$ for a given observed data $\mathrm{D}$ and $B$ for some hypothesis $H$ one want to assess. This allows to write

$$
p(H \mid \mathbf{D}, I)=\frac{p(\mathbf{D} \mid H, I) p(H \mid I)}{p(\mathbf{D} \mid I)} .
$$

Let us now present an interpretation to each one of the terms involved in this equality. The left-hand-side, $p(H \mid \mathbf{D}, I)$ is the posterior probability distribution function (posterior $\mathrm{PDF}$ ) of the hypothesis taking the data into account. On the right-hand-side one has that the posterior PDF is proportional to the sampling PDF of the data $p(\mathbf{D} \mid H, I)$ assuming the hypothesis is true, times the prior PDF for the hypothesis, $p(H \mid I)$, which represents the state of knowledge before the data is taken into account. Note that this is the only PDF that does not depend on the data. On the other hand, the sampling PDF is a fundamental quantity, because it encodes how the degree of plausibility (belief) of the hypothesis changes when one is acquiring new data. Considered as a function of the hypothesis, for fixed data (the one that have been observed), it is called the likelihood function and it is common in literature to employ the notation

$$
\mathcal{L}(H):=p(\mathbf{D} \mid H, I)
$$

It is important however to keep in mind that, as a function of the hypothesis, the likelihood function is not a probability distribution. Up to this point one can see the power of the Bayes' theorem, as long as it specifies the way in which one can update the degree of belief on some hypothesis from experience, i.e., by using observed data. One begins with a prior PDF that does not depend on the data and then fully specifies the degree of belief on the hypothesis before the data is taken into account. Then, when data is wanted to be taken into account, Bayes' theorem dictates the way it should be done, basically by multiplying by the likelihood function associated with the hypothesis.

The left normalization constant in the denominator of the right-hand-side is defined as the Bayesian evidence and is given by the marginalization of the product of the prior and the likelihood over all possible hypothesis,

$$
p(\mathbf{D} \mid I)=\sum_{H} p(\mathbf{D} \mid H, I) p(H \mid I) .
$$

As is easily seen, this results from requiring the posterior PDF to be correctly normalized to the unity or equivalently from satisfying the sum rule for probabilities, equation (3.1). The Bayesian evidence, equation (3.7), is the central quantity for Bayesian model comparison purposes, a topic that is outside of the scope of the present work, so not much will be said about this quantity, however the reader can be referred to e.g. [58] for some discussion. On the other hand, the posterior PDF is the relevant quantity for Bayesian parameter inference as it represents the state of belief about hypothesis after one has considered the information in the data, so it is the central quantity for the proposes of the present work. 


\subsection{Bayesian parameter inference}

This section is intended to depict the Bayesian parameter inference problem. We again follow closely the review of [54] which can be seen for further discussions. In principle, one can formulate the problem of Bayesian parameter inference as follows. Initially, a model should be chosen containing a set of hypotheses in the form of a vector of parameters $\boldsymbol{\theta}$ in the context of the present work, such a model is the standard cosmological model (see the section 1.1). The parameters should describe any aspect of the model, but usually they will represent some physically meaningful quantity inside physical theories. Together with the model, the priors for the parameters should be specified. As was discussed, priors should summarize the state of knowledge about the parameters before considering new data, and the logical way to proceed is then, for the parameter inference step, to consider as prior the posterior PDF obtained from previous data.

The central step is to construct the likelihood function for the measurement, which should reflect the way the data is obtained. Nuisance parameters related to the measurement process might be present in the likelihood. This reflects one of the powerful aspects of Bayesian inference as long as the general strategy is always to work out the joint posterior for all of the parameters in the problem and then marginalize over the ones we are not interested in.

Begin then by assuming a set of model's parameters $\boldsymbol{\theta}$ and a set of nuisance parameters $\boldsymbol{\psi}$, the joint posterior probability for $\boldsymbol{\Theta}=(\boldsymbol{\theta}, \boldsymbol{\psi})$ is obtained through Bayes' Theorem (3.4) as

$$
p(\boldsymbol{\Theta} \mid \mathbf{D}, \mathcal{H})=\frac{\mathcal{L}(\boldsymbol{\Theta}) p(\boldsymbol{\Theta} \mid \mathcal{H})}{p(\mathbf{D} \mid \mathcal{H})}
$$

where an explicit assumption of a model $\mathcal{H}$ was made. For the proposes of parameter inference, the normalizing constant $p(\mathbf{D} \mid \mathcal{H})$, i.e., the Bayesian evidence, can be effectively ignored because it is irrelevant, it only represents a normalization constant factor.

Then it is possible to write the marginal posterior on the parameter of interest by using equation (3.3), i.e., marginalizing over the nuisance parameters,

$$
p(\boldsymbol{\theta} \mid \mathbf{D}, \mathcal{H}) \propto \int \mathrm{d} \boldsymbol{\psi} \mathcal{L}(\boldsymbol{\theta}, \boldsymbol{\psi}) p(\boldsymbol{\theta}, \boldsymbol{\psi} \mid \mathcal{H}) .
$$

The final inference on $\boldsymbol{\theta}$ from the posterior can then be communicated either by some summary statistics, such as the mean, the median or the mode of the distribution, its standard deviation and the correlation matrix among the components, or more usefully (especially for cases where the posterior presents multiple peaks or heavy tails) by plotting one or two dimensional subsets of $\boldsymbol{\theta}$, with the other components marginalized over. On the present work specialized numerical codes for do these kind of jobs was designed so we present as much information as possible for the final inference on parameters.

In general, actual problems in cosmology and astrophysics are not analytically tractable and one must resort to numerical techniques to evaluate the likelihood and to draw samples from the posterior, which constitutes the fundamental problem for parameter inference as long as it encodes all that the data have to tell in the context of a given model. It is then desired to construct an accurate numerical approximation to the posterior PDF following the procedure exposed above. The natural problem that appears is related to the fact that likelihood evaluations can be computationally expensive. For example, calculating the power spectrum of matter fluctuations today, the fundamental quantity to describe the large-scale observables discussed in $\S 1.4$ and/or the Cosmic Microwave 
Background (CMB) power spectrum, basic ingredients to construct likelihood functions associated with its respective observations, at one set of cosmological parameters requires us to evolve many coupled differential equations from inflation to the present day, which can take on the order of seconds to evaluate [28].

The alternatives to solve this problem nowadays are highly influenced by the so-called Markov chain Monte Carlo (MCMC) class of sampling algorithms. This work focus on MCMC samplers, which are briefly depicted in the following section.

\subsection{MCMC techniques for model parameter Bayesian inference}

The present section aims to discuss the most important features of MCMC sampling methods, it is based on the treatment of [53], and does not intend to be complete.

Monte Carlo (MC) methods are computational techniques that make use of random numbers with the aims of solve one or both of the following two problems: (a) to generate samples $\left\{\boldsymbol{\Theta}^{(r)} \mid r \in\{0,2, \ldots, R-1\}\right\}$ from a given probability distribution, for example the posterior probability distribution on the Bayesian inference context $p(\boldsymbol{\Theta} \mid \mathbf{D}, \mathcal{H})$ (sampling problem) and (b) to estimate expectations of functions under this distribution, for example

$$
F:=\langle f(\boldsymbol{\Theta})\rangle=\int \mathrm{d} \boldsymbol{\Theta} p(\boldsymbol{\Theta} \mid \mathbf{D}, \mathcal{H}) f(\boldsymbol{\Theta}) .
$$

A very interesting property of these methods is that one can concentrate only on the sampling problem in order to solve the both. In fact, once sampling process is completed, one can solve the second by using the random samples to give the estimator

$$
\widehat{F}=\frac{1}{R} \sum_{r=1}^{R} f\left(\boldsymbol{\Theta}^{(r)}\right) .
$$

This estimator can be shown to be optimal in the sense that, as the number of samples $R$ increases, the variance of $\widehat{f}$ will decrease as $\sigma_{f}^{2} / R[53]$.

MCMC methods are MC methods where the sampling is made by a sequence of points in parameter space (called "a chain"), of a specific type, a Markov Chain, with density proportional to the posterior PDF of Bayes' Theorem (3.8). A Markov chain is defined as a sequence of random variables $\left\{X^{(t)} \mid t \in\{0,1, \ldots, T-1\}\right\}$ such that the probability of the $(t+1)$-th element in the chain only depends on the value of the $t$-th element. The crucial property of Markov chains is that they can be shown to converge to a stationary state (i.e., which does not change with $t$ ) where successive elements of the chain are samples from the target distribution, in our case, the posterior $\operatorname{PDF} p(\boldsymbol{\Theta} \mid \mathbf{D}, \mathcal{H})$.

Another relevant property of MCMC techniques is that they make the process of obtain and plot marginal posterior PDF for parameters relatively easy. for example if the desired task is to obtain the marginalization to the PDF of a single parameter, say $\theta_{i}$, one can use the MC estimators (3.11). In fact, the process consist only on dividing the range of $\theta_{i}$ in a series of bins and count the number of samples falling within each bin, simply ignoring the coordinates values $\theta_{j}$ with $j \neq i$. The generalization to higher dimensional posteriors, 2-dimensiona, 3-dimensional and so on is enterely analogous.

In what follows we briefly review specific algorithms to perform MCMC sampling used on the present work. For further discussion the reader is encouraged to see the original references cited along the text. 


\subsubsection{Metropolis-Hastings sampling}

The Metropolis-Hastings (MH) algorithm is one of the most widely used MCMC sampling methods for Bayesian inference. It was introduced by [55] as a way to solve the interacting Ising model in the lattice, see e.g. [56].

$\mathrm{MH}$ algorithm makes use of a proposal (also called in the literature by trial) density, $q\left(\boldsymbol{\Theta}^{\prime} ; \boldsymbol{\Theta}^{(t)}\right)$, which depends on the current state of the chain (point in parameter space for parameter inference proposes) $\boldsymbol{\Theta}^{(t)}$ and essentially describes the probability for at a definite step, say the $t$-th one, the chain goes from the current state $\boldsymbol{\Theta}^{(t)}$ to a new state $\boldsymbol{\Theta}^{\prime}$. The density PDF $q$ can be any fixed density from which one can draw samples. Common choices are based on simplicity, for example, a Gaussian centered on the current state $\Theta^{(t)}$.

The essentials of the $\mathrm{MH}$ method to sample the PDF $p(\boldsymbol{\Theta})$ can be summarized as follows: first of all, choose an initial point in parameter space $\boldsymbol{\Theta}^{(0)}$, then, each MH step to evolve the chain can be represented on pseudo-code according to algorithm 1 . Hereinafter

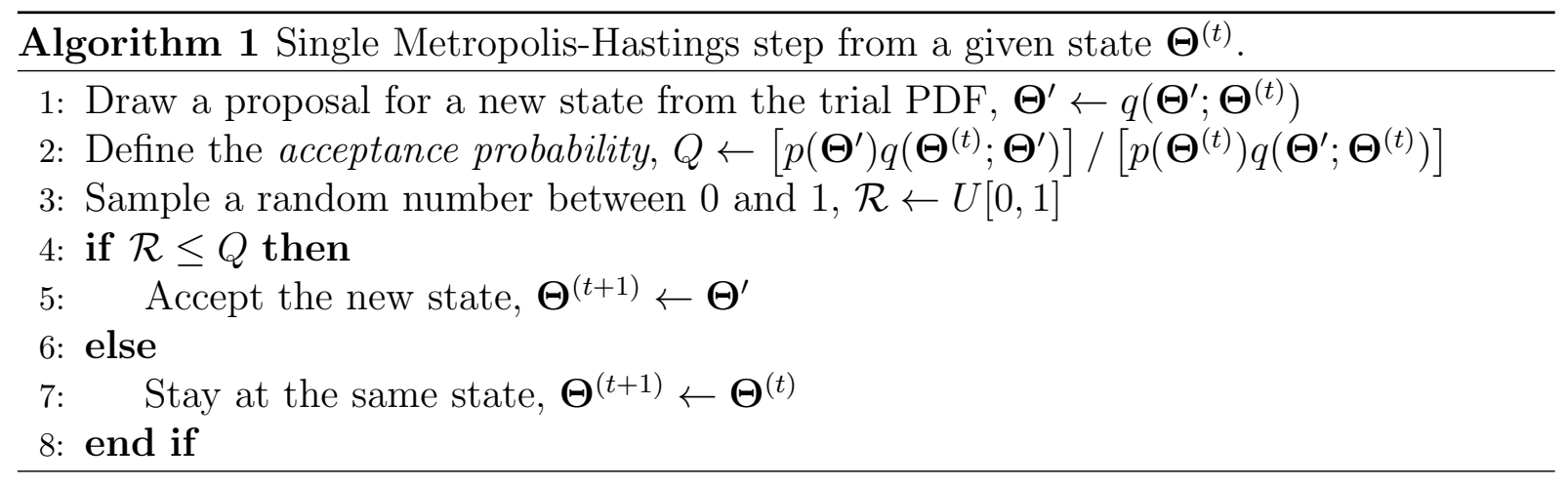

$U[0,1]$ denotes a uniform PDF between 0 and 1 .

It is important to clarify notation. We use the superscript $r=1, \ldots, R$ to label points that are independent samples from a distribution, and the superscript $t=1, \ldots, T$ to label the sequence of states in a Markov Chain. It is important to note that a MetropolisHastings simulation of $T$ iterations do not produce $T$ independent samples from the target distribution $P$. The samples are, in principle, dependent.

Note that in order to compute the acceptance probability $Q$ it is necessary to compute the probability ratios $p\left(\boldsymbol{\Theta}^{\prime}\right) / p\left(\mathbf{\Theta}^{(t)}\right)$ and $q\left(\boldsymbol{\Theta}^{(t)} ; \boldsymbol{\Theta}^{\prime}\right) / q\left(\boldsymbol{\Theta}^{\prime} ; \mathbf{\Theta}^{(t)}\right)$, line 2 in algorithm 1 . In the special case in which the proposal density is chosen to be a simple symmetrical density such as a Gaussian centered on the current point, the later factor is unity and the MH method simply involves comparing the value of the target density at the two points. This special case is sometimes called the Metropolis method, because it was in fact the one originally introduced by Metropolis [55]. It is important to mention that the symmetry of the trial distribution $q$ guarantees the stationarity of $p(\boldsymbol{\Theta})$ under the Markov process and thus that the asymptotic distribution of the chain is effectively $p(\boldsymbol{\Theta})$ [53].

\subsubsection{Affine-invariant ensemble MCMC}

Recently a new class of MCMC methods had received much attention from the community, the ensemble or many-particle MCMC sampling algorithms introduced by [59,60]. Such class of algorithms have the property of affine-invariance; that is, the performance of the algorithm is invariant under linear transformations of the parameter space (hence the name). This is a highly desirable property because if it is guaranteed, in principle, one 
of this algorithms can work equally well sampling an uncorrelated and isotropic Gaussian and a highly degenerate target distribution [57].

The basic principle of this sampling methods is that, in contrast to common $\mathrm{MH}$ method, where a chain is generated sequentially as a single point (particle) moving on parameter space, many points (particles), commonly called as walkers, explore, at the same time, the parameter space. Moreover, this principle allows one to think in schemes of parallelization of this kind of methods.

Following [57], the essentials of an affine-invariant MCMC sampling algorithm can be described as follows: first of all an initial position for each of the walkers should be chosen, i.e., an initial ensemble configuration $(t=0)$. An ensemble of $K$ walkers will be denoted as

$$
S:=\left\{\boldsymbol{\Theta}_{k} \mid k \in\{0,1, \ldots, K-1\}\right\} .
$$

The proposal distribution for one walker, say the $k$-th one, is based on the current positions of the $K-1$ walkers on the complementary ensemble,

$$
S_{[k]}:=\left\{\Theta_{j} \mid j \neq k\right\} .
$$

This move should be done in such a way that detailed balance holds, imposing a restriction on the possible types of moves (see discussion in [59]). Here the simplest one will be discussed, in fact the one that is used during this work: the so called stretch move. In the stretch move, in order to update the position of one walker $\boldsymbol{\Theta}_{k}$ a walker $\boldsymbol{\Theta}_{j}$ is drawn randomly from the complementary ensemble, $\boldsymbol{\Theta}_{j} \in S_{[k]}$, and a new position is proposed according to

$$
\boldsymbol{\Theta}^{\prime}=\boldsymbol{\Theta}_{j}+Z\left[\boldsymbol{\Theta}_{k}(t)-\boldsymbol{\Theta}_{j}\right],
$$

where $Z$ is a random variable drawn from a PDF $g(z)$. In this sense, according to the proposed position, one has that

$$
\boldsymbol{\Theta}_{k}(t)=\boldsymbol{\Theta}_{j}+Z^{-1}\left[\Theta^{\prime}-\Theta_{j}\right]
$$

this shows that the proposal is symmetric, in the sense that the probability to go to a position has the same value of the probability to return from it, if the PDF $g$ satisfies the condition

$$
g\left(z^{-1}\right)=z g(z)
$$

A particular form of $g(z)$ commonly used was originally proposed by [60] as

$$
g(z) \propto \begin{cases}z^{-1 / 2}, & z \in\left[a^{-1}, a\right] \\ 0, & \text { otherwhise }\end{cases}
$$

where the parameter $a>1$, controlling the step size, can be adjusted to improve performance. Following [57] this parameter is setted in this work to $a=2$ for all practical applications. However, is important to remark the fact that in principle $a$ may be varied if the acceptance fraction of the sampling process is too low or too high (see [59] and [57] for discussion).

Finally, the proposal is accepted following the acceptance probability

$$
Q=\min \left(Z^{n-1} \frac{p\left(\boldsymbol{\Theta}^{\prime}\right)}{p\left(\boldsymbol{\Theta}_{k}(t)\right)}\right),
$$

where $n$ is the dimension of the parameter space. The above form of the acceptance probability $Q$ in conjunction with the symmetry condition imposed to the probability 
density $g$ of the trial step, equation (3.17), ensures detailed balance and therefore that the asymptotic distribution of the Markov Chains generated by the ensemble of walkers is in fact the target PDF $p[59,60]$.

The single stretch move is summarized in form of pseudo-code on algorithm 2.

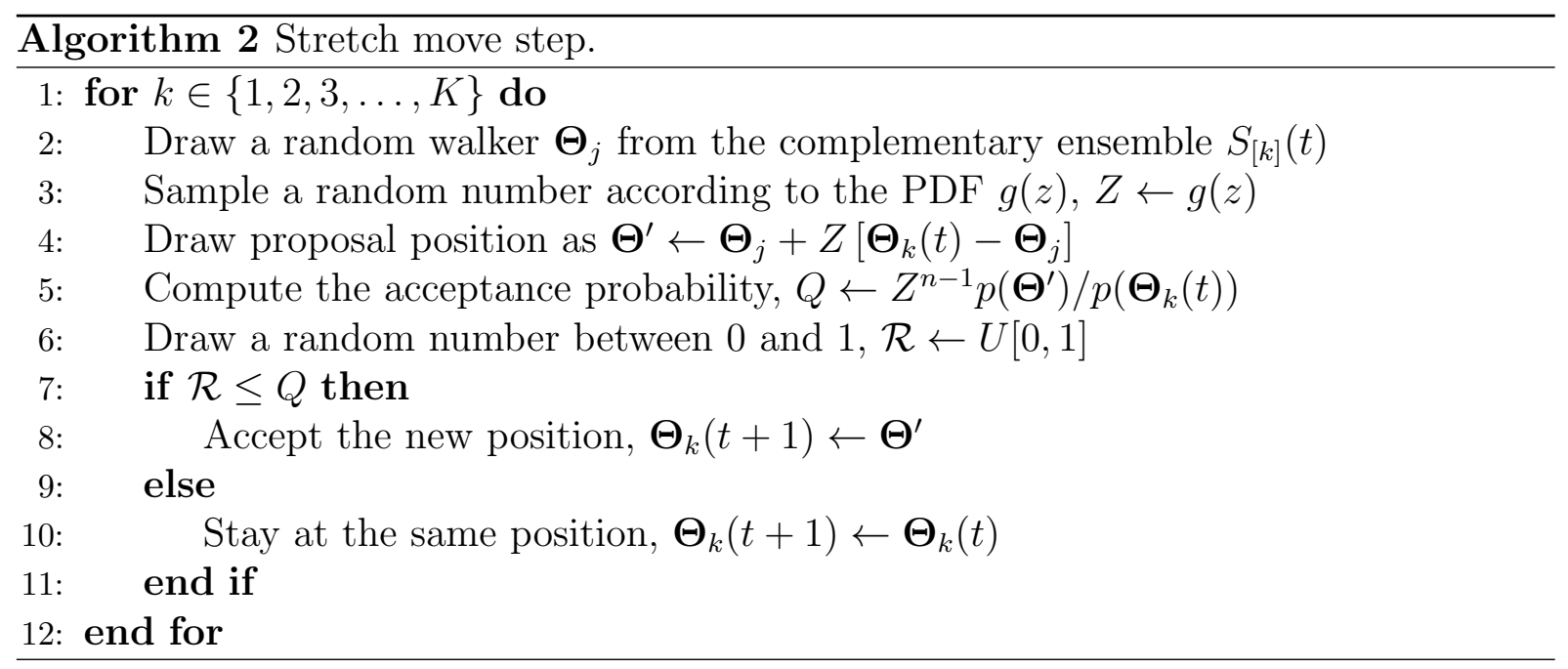

Perhaps the most attractive feature of the affine-invariant ensemble method for MCMC sampling based on the stretch move, algorithm 2, is the fact that it admits an scheme of parallelization. As discussed in [57] this parallelization should carefully done, the first natural thought of parallel advancing each walker based on the state of the ensemble instead of evolving the walkers in series, as is implicit on algorithm 2, is not allowed because it subtly violates detailed balance. However a scheme of parallelization not violating detailed balance is also given in [57]. The solution resides in split the full ensemble into two subsets (sub-ensembles if you prefer),

$$
S^{(0)}:=\left\{\boldsymbol{\Theta}_{k} \mid k \in\{1,2,3, \ldots, K / 2\}\right\}, \quad S^{(1)}:=\left\{\boldsymbol{\Theta}_{k} \mid k \in\{K / 2+1 \ldots, K\}\right\} .
$$

Thus, first update all the walkers in the first sub-ensemble $S^{(0)}$ using the stretch move procedure from algorithm 2 based only on the positions of the walkers in the other subensemble $S^{(1)}$, and then, using the new positions of $S^{(0)}$, update $S^{(1)}$. This procedure is summarized in the form of pseudocode in Algorithm 3.

In this way, as can be seen from algorithm 3 the computationally expensive inner loop, which starts at line 2 in algorithm 3, can efficiently run in parallel. A highly desired property nowadays when parallel computing is becoming common practice for scientific community. Given the variety of properties just depicted about affine-invariant ensemble MCMC samplers, we used them during this work, for practical proposes we used the emcee $^{1}$ [57] code, a python-based code designed to be a "hammer" for MCMC sampling.

\footnotetext{
${ }^{1}$ https://github.com/dfm/emcee
} 


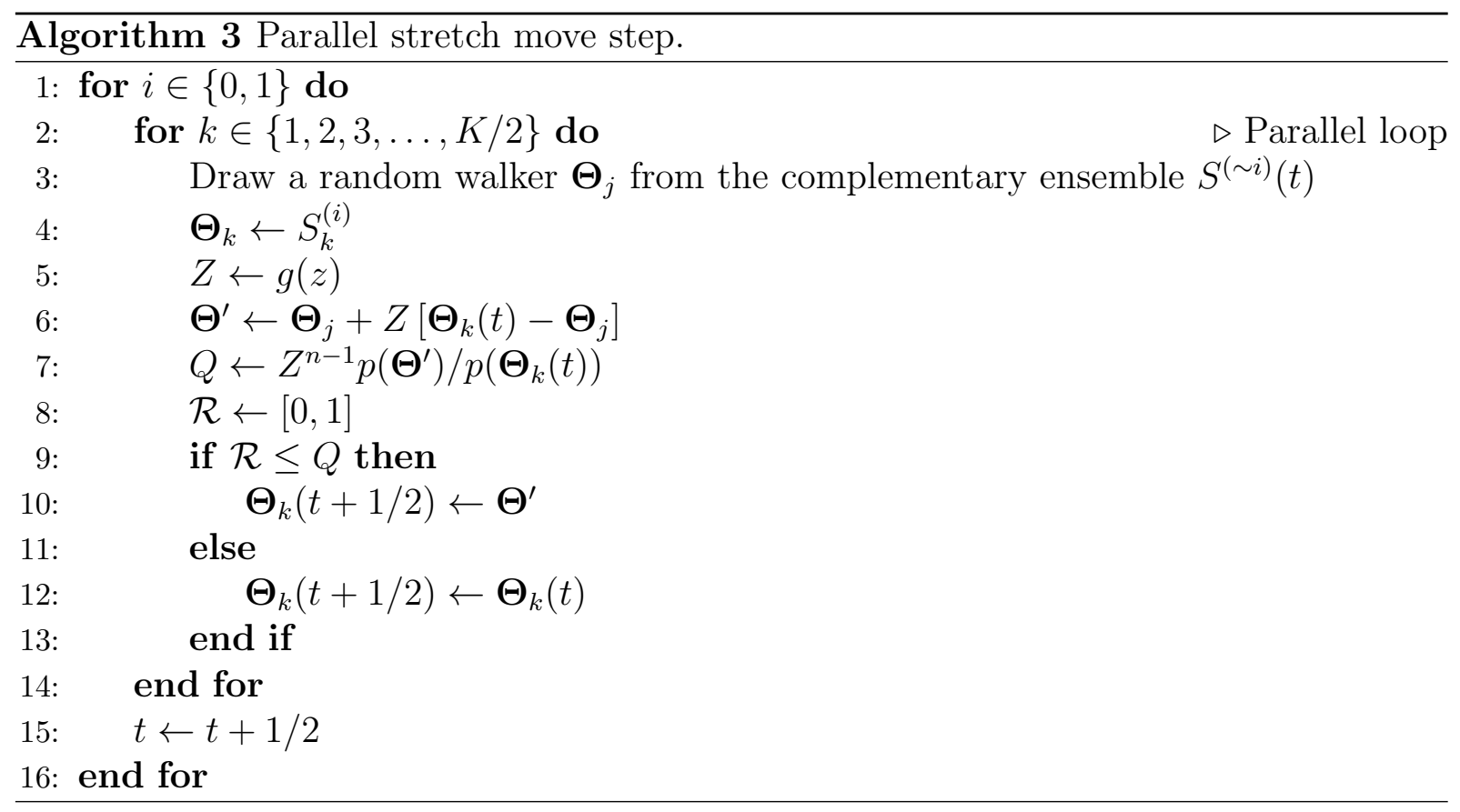




\section{Chapter 4}

\section{Results}

This chapter is devoted to the main results of this work. We begin by considering the general two-point correlation function of galaxies for arbitrary angular separation and redshifts. This is accomplished by using the results presented in $\S 2.1$ and including the redshift-space distortion effect as discussed in Appendix C, such that a general formula for arbitrary spatial curvature is presented. Next, the distant observer approximation is discussed and the standard functional forms of the two-point correlation function are recovered. In order to do so, we closely follow the treatment of [35].

Interpretation of data from galaxy redshift surveys relies on the comparison of observations and theoretical predictions. Modern approaches rely on numerical computations in the linear regime performed by sophisticated Boltzmann codes, i.e. numerical routines specialized in evolving the Einstein-Boltzmann system of equations associated with firstorder metric perturbations, taking into account the interaction between different species in the Universe according to the formalism of Kinetic Theory [2, 4].

Two main difficulties appear in this interpretation, (a) whether the model postulated is a correct physical description of the Universe evolution and (b) given a model, whether the theoretical predictions are computed with sufficient accuracy when the observational errors are taken into account. The first problem can be addressed by a continuous theoretical effort and evaluation of how well competing models describe the same data. This can be done by using Bayesian inference as described in Chapter 3. On the other hand, the second difficulty is directly related to the robustness of the numerical methods used to compute predictions from models. Commonly these methods are divided into two specific tasks, Einstein-Boltzmann codes and specific observable model codes, i.e. the numerical methods used to connect the solutions offered by the linear theory codes for the evolution of perturbations in the Universe with the actual cosmological observables as e.g. those discussed in section 1.4. On top of that, practical progress in the development of these codes is triggered by the fact that their speed is crucial for sampling the space of model parameters. As discussed in chapter 3, within the Bayesian inference process, sampling methods require the evaluation of a considerable number of models every time a cosmology is compared to the observed data set.

For the Einstein-Boltzmann system of equations, several Boltzmann codes have been made public and compared to each other. From them, we can mention CMBFAST ${ }^{1}$ [61, 62], the first numerical routine implementing the method of line-of-sight integration written in Fortran 77 language and not maintained up to date, and later CAMB $^{2}$ [28], a Fortran

\footnotetext{
${ }^{1}$ http://lambda.gsfc.nasa.gov/toolbox/tb_cmbfast_ov.cfm

${ }^{2}$ http://camb.info/
} 
90 implementation of the same method which is maintained up to date, CMBEASY [63] ${ }^{3}$ an early $\mathrm{C}++$ (object oriented) implementation based on CMBFAST, not maintained up to date, and more recently $\operatorname{CLASS}^{4}$ [64, 65], a C++ implementation maintained up to date. From all of them, CAMB and CLASS have been constantly updated and pushed to ever higher precision and accuracy.

In this chapter, different approaches to the practical computation of the two-point correlation function assuming the distant observer approximation are considered. We show that a method based on discrete Hankel transform becomes competitive and nearly optimal in the sense of being accurate and faster in comparison to standard integration methods.

In the context of modern wide-area photometric galaxy surveys like the DES, the gain in area and depth should be contrasted with a poorer determination of radial positions. Therefore, splitting the data into redshift bins and using the angular correlation function $(\mathrm{ACF}) w(\theta)$ and the angular power spectrum (APS) $C_{\ell}$ constitute a standard approach to extract cosmological information. We present a detailed model for $w(\theta)$ at large scales including effects of nonlinear gravitational clustering, galaxy biasing, redshift space distortions and photo- $z$ uncertainties.

In the same context, future cosmic shear surveys will play a major role in cosmology. The sensitivity of weak lensing observations will be high enough to allow for precision measurements of cosmological parameters. These measurements will provide constraints on cosmology that are independent and complementary to those obtained from other observables such as galaxy clustering, CMB anisotropies and polarization, type Ia supernovae and galaxy clusters [1]. Cosmic shear probes the nonlinear evolution of structures in the Universe and can be used to measure the non-Gaussianity of the large scale structure arising from gravitational collapse. Up to now, cosmic shear observations have focused mainly on second-order (two-point) statistics which only probe the Gaussian part of the matter distribution. Higher-order statistics that are able to determine non-Gaussian aspects of the large scale structure will become more important and observationally feasible with larger and deeper surveys, higher sensitivity and better understanding of systematics in the measurements and in the data analysis [11, 10]. Moreover, since the dependence on cosmology is different for shear statistics of second- and third-order, the combination of both will improve the determination of cosmological parameters and reduce the degeneracies between them. In the final part of this chapter, preliminary work on the galaxy position and weak lensing convergence auto- and cross- correlation in harmonic space, i.e. the angular power spectra, for the Onion simulations [13] is also presented.

\subsection{Two-point statistics in configuration space}

In $§ 2.1 .1$ the two-point correlation function of total matter fluctuations $\xi(r)$ in FLRW Universe models was found in terms of the total matter power spectrum according to equations (2.15) and (2.21). In this section those expressions will be generalized to the case of the two-point correlation function of galaxies, equation (1.59), closely following the pioneering work of [35]. After doing that we show how to use this result to construct the angular two point correlation function of galaxies. We provide expressions that take into account spatial curvature, nonlinear corrections, photometric redshift estimation errors

\footnotetext{
${ }^{3}$ http://www.thphys. uni-heidelberg.de/ robbers/cmbeasy/

${ }^{4}$ http://class-code.net/
} 
and the proper covariance between redshift and angular scales, this is done by closely follow the works in $[7,8]$.

\subsubsection{Galaxy two-point correlation function in FLRW Universes}

By properly accounting for the effect of the peculiar velocities of galaxies on the fluctuation in their number density as discussed in $\$ 1.4 .1$ and computed in Appendix C one can write the two-point correlation function of galaxies in redshift space as

$$
\xi_{\mathrm{gg}}^{(\mathrm{rs})}\left(z_{1}, x_{1}^{i}, z_{2}, x_{2}^{i}\right)=\left\langle\delta_{\mathrm{g}}^{(\mathrm{rs})}\left(z_{1}, x_{1}^{i}\right) \delta_{\mathrm{g}}^{(\mathrm{rs})}\left(z_{2}, x_{2}^{i}\right)\right\rangle=b_{1} b_{2} G_{1} G_{2}\left(\widehat{R}_{1} \circ \widehat{R}_{2}\right)[\xi(\chi)]
$$

where $b_{i}:=b_{\mathrm{g}}\left(z_{i}\right), G_{i}=G\left(z_{i}\right)$ with $z_{i}$ for $i=1,2$ the redshifts of the two points where the galaxies are located, $\xi(\chi)$ is the total matter two-point correlation function at present time in terms of the adimensional radial separation between the two points $\chi$ (equation (2.15)) and the two redshift-space distortion operators $\widehat{R}_{1}$ and $\widehat{R}_{2}$ (equation (C.36)) $)^{5}$ are applied on the total matter fluctuation at the spatial points $x_{1}^{i}$ and $x_{2}^{i}$ respectively.

In the following, explicit expressions for the correlation function in redshift space are derived. The approach is to separately consider open, flat and closed Universes following the work of [35]. However, from equation (4.1) one can see that the main task is to compute the action of the composition of redshift-space distortion operators $\widehat{R}_{1} \circ \widehat{R}_{2}$ on the real space correlation function of matter at present time $\xi(\chi)$. Regardless of the spatial curvature, by simple inspection of equations (2.15) and (C.36), one see that this computation involves the action of the inverse of the operator $\left(\nabla_{1,2}^{2}+3 K\right)$ on the zero radial harmonic modes $X_{0}$ (equations (B.23)). More explicitly, the action of the composition $\left(\nabla_{1}^{2}+3 K\right)^{-1} \circ\left(\nabla_{2}^{2}+3 K\right)^{-1}$ on $X_{0}$. Such computation can always be done by taking into account the facts that the Laplacian operator is invariant under changes of coordinates in the spatial hypersurfaces and that the $X_{0}$ functions are special cases of the Laplace operator eigenfunctions (see Appendix B). Then we should have

$$
\left(\nabla_{1}^{2}+3 K\right)^{-1}\left(\nabla_{2}^{2}+3 K\right)^{-1} X_{0}(\chi, \nu)=\frac{X_{0}(\chi, \nu)}{\left(3 K-k^{2}\right)^{2}}
$$

where $k$ is the dimensional eigenvalue of the Laplace operator related to its adimensional analog $\nu$ depending on the spatial curvature according to equations (B.5). The remaining part of the computation involves derivatives along the LOS, i.e. derivatives with respect to $\chi_{i}=\chi\left(z_{i}\right)(i=1,2)$, equation (1.19), which are non-trivial because they do not have the invariance property of the Laplacian. This computations can then be performed by introducing the angular separation between the two points $\theta$ and the angles subtended between the lines of sight to each point and the geodesic connecting the two points $\gamma_{i}(i=$ 1,2 ), see figure 4.1. Since these definitions are geometry (spatial curvature)-dependent they are separated for open, flat and closed FLRW Universe models.

\section{Open FLRW Universe}

Consider initially an open Universe, i.e., $K<0$. The two-point correlation function of total matter at present time is given by (see $\S 2.1 .1$ )

$$
\xi(\chi)=\int_{0}^{\infty} \frac{\mathrm{d} \nu \nu^{2}}{2 \pi^{2}} X_{0}^{(-)}(\chi, \nu) S(\nu) ; \quad X_{0}^{(-)}(\chi, \nu)=\frac{\sin (\nu \chi)}{\nu \sinh (\chi)},
$$

\footnotetext{
${ }^{5}$ Note that here the subindex $g$ has been dropped out for simplicity in the notation
} 


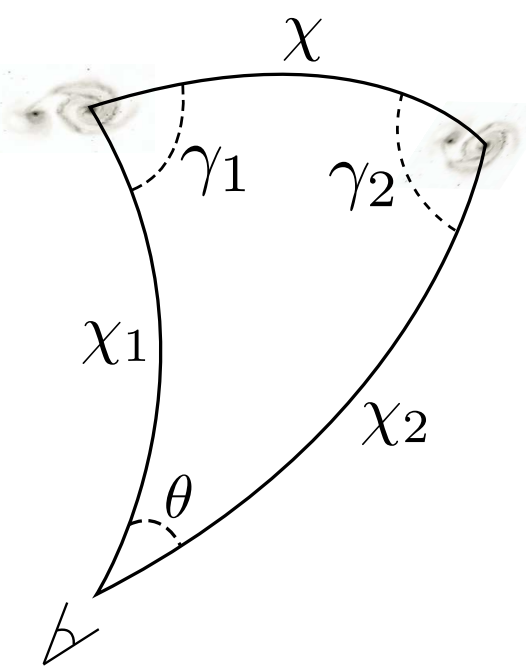

Figure 4.1: Geometry of the two-point correlation function in FLRW Universe models. The two points are supposed to have spacetime coordinates $\left(\eta_{1,2}, x_{1,2}^{i}\right)$. Since the points should lie on the observer past light cone the time coordinate can be replaced by the redshift $z_{1,2}$ used on the definition of the correlation of galaxies (4.1). Choosing spatial spherical coordinates $x^{i}=(\chi, \theta, \varphi)$ the radial positions of the two points is given by $\chi_{1,2}=\chi\left(z_{1,2}\right)$, the radial distance between the objects $\chi$ depends on the spatial geometry (curvature), $\theta$ is the angle subtended by the two objects for the observer and $\gamma_{1,2}$ are the angles between the lines of sight two the objects and the geodesic connecting them.

where $S(\nu)$ is the adimensional total matter power spectrum related to its fully dimensional analog $P(k)$ according to equation (2.17). Expanding the composition of the two redshift-space distortion operators $\left(\widehat{R}_{1} \circ \widehat{R}_{2}\right)$ for the terms that involve derivatives along the LOS, one has

$$
\begin{aligned}
\left(\widehat{R}_{1} \circ \widehat{R}_{2}\right)= & {\left[1+\beta_{1} K\left(\partial_{1}+\alpha_{1}\right) \partial_{1}\left(\nabla_{1}^{2}+3 K\right)^{-1}\right]\left[1+\beta_{2} K\left(\partial_{2}+\alpha_{2}\right) \partial_{2}\left(\nabla_{2}^{2}+3 K\right)^{-1}\right] } \\
= & \left(1+K \beta_{1} \alpha_{1} \partial_{1}+K \beta_{2} \alpha_{2} \partial_{2}+K \beta_{1} \partial_{1}^{2}+K \beta_{2} \partial_{2}^{2}\right. \\
& +K^{2} \beta_{1} \beta_{2} \alpha_{1} \alpha_{2} \partial_{1} \partial_{2}+K^{2} \beta_{1} \beta_{2} \alpha_{2} \partial_{1}^{2} \partial_{2}+K^{2} \beta_{1} \beta_{2} \alpha_{1} \partial_{1} \partial_{2}^{2} \\
& \left.+K^{2} \beta_{1} \beta_{2} \partial_{1}^{2} \partial_{2}^{2}\right)\left(\nabla_{2}^{2}+3 K\right)^{-1}\left(\nabla_{2}^{2}+3 K\right)^{-1}
\end{aligned}
$$

where the abbreviated notation $\beta_{i}:=\beta_{\mathrm{g}}\left(z_{i}\right), \alpha_{i}:=\alpha\left(\chi_{i}\right), \partial_{i}:=\partial_{\chi_{i}}$ was introduced for simplicity. The action of the inverse Laplace operators on the $\ell=0$ radial mode is given by equation (4.2) as

$$
\left(\nabla_{2}^{2}+3 K\right)^{-1}\left(\nabla_{2}^{2}+3 K\right)^{-1} X_{0}^{(-)}(\chi, \nu)=\frac{1}{K^{2}} \frac{1}{\left(\nu^{2}+4\right)} X_{0}^{(-)}(\chi, \nu) .
$$

As discussed above, the non-trivial task is now to compute the derivatives along lines-ofsight of the two points.

We start by using the open-space version of the law of cosines to relate the radial positions of the two objects $\chi_{i}$ and its angular separation $\theta$, as determined by the observer, with their comoving separation $\chi$ (see figure 4.1),

$$
\cosh (\chi)=\cosh \left(\chi_{1}\right) \cosh \left(\chi_{2}\right)-\sinh \left(\chi_{1}\right) \sinh \left(\chi_{2}\right) \cos (\theta)
$$

Then the angles subtended between the lines of sight to the objects (galaxies) at $\chi_{i}$ and 
the geodesic connecting these objects $\chi$ can be computed as

$$
\begin{aligned}
& \cos \left(\gamma_{1}\right)=\frac{\partial \chi}{\partial \chi_{1}}=\frac{1}{\sinh (\chi)}\left[\sinh \left(\chi_{1}\right) \cosh \left(\chi_{2}\right)-\cosh \left(\chi_{1}\right) \sinh \left(\chi_{2}\right) \cos (\theta)\right] \\
& \cos \left(\gamma_{2}\right)=\frac{\partial \chi}{\partial \chi_{2}}=\frac{1}{\sinh (\chi)}\left[\cosh \left(\chi_{1}\right) \sinh \left(\chi_{2}\right)-\sinh \left(\chi_{1}\right) \cosh \left(\chi_{2}\right) \cos (\theta)\right] .
\end{aligned}
$$

In order to find the partial derivative of the $\gamma_{1,2}$ angles along the lines of sight it is also useful to consider the following identities hold [35]

$$
\begin{gathered}
\frac{\partial}{\partial \chi_{1}}\left(\sinh \chi \cos \gamma_{1}\right)=\cosh \chi \\
\frac{\partial}{\partial \chi_{2}}\left(\sinh \chi \cos \gamma_{1}\right)=-\cosh \chi \cos \tilde{\theta},
\end{gathered}
$$

where the angle $\tilde{\theta}$ is introduced according to

$$
\cos \tilde{\theta}=\frac{\sin \gamma_{1} \sin \gamma_{2}}{\cosh \chi}-\cos \gamma_{1} \cos \gamma_{2}=\frac{\cosh \chi_{1} \cosh \chi_{2} \cos \theta-\sinh \chi_{1} \sinh \chi_{2}}{\cosh \chi_{1} \cosh \chi_{2}-\sinh \chi_{1} \sinh \chi_{2} \cos \theta} .
$$

Note from this definition that $\theta \rightarrow \tilde{\theta}$ for comoving scales much smaller than the curvature scale, i.e. in the flat limit, $r \ll|K|^{-1 / 2}$ or equivalently $\chi \ll 1$. These identities allow to write the derivatives of the $\gamma_{1,2}$ angles with respect to $\chi_{1,2}$ as

$$
\frac{\partial \cos \left(\gamma_{i}\right)}{\partial \chi_{j}}= \begin{cases}\operatorname{coth}(\chi)\left[1-\cos ^{2}\left(\gamma_{i}\right)\right] & i=j \\ -\operatorname{coth}(\chi)\left[\cos (\tilde{\theta})+\cos \left(\gamma_{i}\right) \cos \left(\gamma_{j}\right)\right] & i \neq j\end{cases}
$$

where $i, j \in\{1,2\}$.

By using the above equations (4.6)-(4.10) and the derivative and recursion relations for the radial harmonic modes, equations (B.10)-(B.11), one can obtain the derivatives along the lines of sight appearing on the expansion (4.4) as

$$
\begin{aligned}
\frac{\partial X_{0}}{\partial \chi_{i}}= & \cos \left(\gamma_{i}\right) X_{1}(\chi, \nu), \\
\frac{\partial^{2} X_{0}}{\partial \chi_{1}^{2}}= & X_{0}-\frac{1}{3}\left(\nu^{2}+4\right) X_{0}+\left[\cos ^{2}\left(\gamma_{1}\right)-\frac{1}{3}\right] X_{2}, \\
\frac{\partial^{2} X_{0}}{\partial \chi_{1} \partial \chi_{2}}= & -\cos (\tilde{\theta}) X_{0}+\frac{1}{3} \cos (\tilde{\theta})\left(\nu^{2}+4\right) X_{0}+\left[\cos \left(\gamma_{1}\right) \cos \left(\gamma_{2}\right)+\frac{1}{3} \cos (\tilde{\theta})\right] X_{2},(4.11 \mathrm{c}) \\
\frac{\partial^{3} X_{0}}{\partial^{2} \chi_{i} \partial \chi_{j}}= & \cos \left(\gamma_{j}\right) X_{1}+\frac{1}{5}\left(\nu^{2}+4\right)\left[2 \cos \left(\gamma_{i}\right) \cos (\tilde{\theta})-\cos \left(\gamma_{j}\right)\right] X_{1} \\
& +\frac{1}{5}\left[2 \cos \left(\gamma_{i}\right) \cos (\tilde{\theta})+5 \cos ^{2}\left(\gamma_{i}\right) \cos \left(\gamma_{j}\right)-\cos \left(\gamma_{j}\right)\right] X_{3}, \\
\frac{\partial^{4} X_{0}}{\partial \chi_{1}^{2} \partial \chi_{2}^{2}}= & X_{0}-\frac{2}{15}\left(4+3 \cos ^{2} \tilde{\theta}\right)\left(\nu^{2}+4\right) X_{0}+\frac{1}{15}\left(1+2 \cos \tilde{\theta}^{2}\right)\left(\nu^{2}+4\right)^{2} X_{0} \\
& -\frac{1}{21}\left[4-6 \cos ^{2} \tilde{\theta}-27\left(\cos ^{2} \gamma_{1}+\cos ^{2} \gamma_{2}\right)-60 \cos \gamma_{1} \cos \gamma_{2} \cos \tilde{\theta}\right] X_{2} \\
& +\frac{1}{21}\left[2+4 \cos ^{2} \tilde{\theta}-3\left(\cos ^{2} \gamma_{1}+\cos ^{2} \gamma_{2}\right)+12 \cos \gamma_{1} \cos \gamma_{2} \cos \tilde{\theta}\right]\left(\nu^{2}+4\right) X_{2} \\
& +\frac{1}{35}\left[1+2 \cos ^{2} \tilde{\theta}-5\left(\cos ^{2} \gamma_{1}+\cos ^{2} \gamma_{2}\right)+20 \cos \gamma_{1} \cos \gamma_{2} \cos \tilde{\theta}\right. \\
& \left.+35 \cos ^{2} \gamma_{1} \cos ^{2} \gamma_{2}\right] X_{4} \cdot
\end{aligned}
$$


Thus the action of the composition of redshift-space distortion operators $\widehat{R}_{1} \circ \widehat{R}_{2}$ on $X_{0}^{(-)}(\chi, \nu)$ can be expanded as [35]

$$
\left(\widehat{R}_{1} \circ \widehat{R}_{2}\right) X_{0}^{(-)}(\chi, \nu)=\sum_{n, \ell} c_{\ell}^{(n)}\left(\chi_{1}, \chi_{2}, \theta\right) \frac{(-1)^{n} X_{\ell}^{(-)}(\chi, \nu)}{\sinh ^{2 n-\ell}(\chi)\left(\nu^{2}+4\right)^{n}},
$$

where the sum runs over $(n, \ell)=(0,0),(1,0),(1,1),(1,2),(2,0),(2,1),(2,2),(2,3),(2,4)$, and consequently the two-point correlation function of galaxies in redshift space is given by (equation (4.1))

$$
\xi_{\mathrm{gg}}^{(\mathrm{rs})}\left(z_{1}, z_{2}, \theta\right)=b_{\mathrm{g}}\left(z_{1}\right) b_{\mathrm{g}}\left(z_{2}\right) G\left(z_{1}\right) G\left(z_{2}\right) \sum_{n, \ell} c_{\ell}^{(n)}\left(\chi_{1}, \chi_{2}, \theta\right) \Xi_{\ell}^{(n)}(\chi),
$$

where the multipoles of the two-point correlation function were introduced as

$$
\Xi_{\ell}^{(n)}(\chi)=\frac{(-1)^{n}}{\sinh ^{2 n-\ell}(\chi)} \int_{0}^{\infty} \frac{\mathrm{d} \nu \nu^{2}}{2 \pi^{2}} \frac{X_{\ell}(\chi, \nu)}{\left(\nu^{2}+4\right)^{n}} S(\nu)
$$

Note that the index $n$ appears to distinguish the $\nu$ dependence on the expansion of $\widehat{R}_{1} \widehat{R}_{2} X^{(-)}(\chi, \nu)$. The actual multipolar expansion runs over the $\ell$ index alone. Note further that since the two points are inside the past light-cone of the observer the expansion coefficients $c_{\ell}^{(n)}$ and consequently the correlation function in redshift space depends only on the redshifts of the two points $z_{1,2}$ and their angular separation $\theta$.

The coefficients $c_{\ell}^{(n)}\left(z_{1}, z_{2}, \theta\right)$ are given according to the line of sight derivatives (equations (4.10)-(4.11d)) as

$$
\begin{aligned}
& c_{0}^{(0)}=1+\frac{1}{3}\left(\beta_{1}+\beta_{2}\right)+\frac{1}{15} \beta_{1} \beta_{2}\left(1+2 \cos ^{2}(\tilde{\theta})\right), \\
& c_{0}^{(1)}=-\frac{1}{3} \beta_{1} \beta_{2} \tilde{\alpha}_{1} \tilde{\alpha}_{2} \cos (\tilde{\theta})+\left[\beta_{1}+\beta_{2}+\frac{2}{15} \beta_{1} \beta_{2}(4+3 \cos (\tilde{\theta}))\right] \sinh ^{2}(\chi) \text {, } \\
& c_{1}^{(1)}=\beta_{1} \tilde{\alpha}_{1} \cos \left(\gamma_{1}\right)+\beta_{2} \tilde{\alpha}_{2} \cos \left(\gamma_{2}\right)+\frac{1}{5} \beta_{1} \beta_{2}\left[\tilde{\alpha}_{1}\left(\cos \left(\gamma_{1}\right)-2 \cos \left(\gamma_{2}\right) \cos (\tilde{\theta})\right)\right. \\
& \left.+\tilde{\alpha}_{2}\left(\cos \left(\gamma_{2}\right)-2 \cos \left(\gamma_{1}\right) \cos (\tilde{\theta})\right)\right] \text {, } \\
& c_{2}^{(1)}=\beta_{1}\left(\cos \left(\gamma_{1}\right)-\frac{1}{3}\right)+\beta_{2}\left(\cos \left(\gamma_{2}\right)-\frac{1}{3}\right)-\frac{1}{7} \beta_{1} \beta_{2}\left[\frac{2}{3}+\frac{4}{3} \cos ^{2}(\tilde{\theta})\right. \\
& \left.-\left(\cos ^{2}\left(\gamma_{2}\right)+\cos ^{2}\left(\gamma_{2}\right)\right)+4 \cos \left(\gamma_{1}\right) \cos \left(\gamma_{2}\right) \cos (\tilde{\theta})\right] \text {, } \\
& c_{0}^{(2)}=\beta_{1} \beta_{2}\left(\sinh ^{2}(\chi)-\tilde{\alpha}_{1} \tilde{\alpha}_{2}\right) \sinh ^{2}(\chi), \\
& c_{1}^{(2)}=\beta_{1} \beta_{2}\left(\tilde{\alpha}_{1} \cos \left(\gamma_{1}\right)+\tilde{\alpha}_{2} \cos \left(\gamma_{2}\right)\right) \sinh ^{2}(\chi), \\
& c_{2}^{(2)}=\beta_{1} \beta_{2} \tilde{\alpha}_{1} \tilde{\alpha}_{2}\left(\cos \left(\gamma_{1}\right) \cos \left(\gamma_{2}\right)+\frac{1}{3} \cos (\tilde{\theta})\right)+\frac{2}{7} \beta_{1} \beta_{2}\left[\cos ^{2}(\tilde{\theta})-\frac{2}{3}\right. \\
& \left.+\frac{9}{2}\left(\cos ^{2}\left(\gamma_{1}\right)+\cos ^{2}\left(\gamma_{2}\right)\right)+10 \cos \left(\gamma_{1}\right) \cos \left(\gamma_{2}\right) \cos (\tilde{\theta})\right] \sinh ^{2}(\chi), \\
& c_{3}^{(2)}=\frac{1}{5} \beta_{1} \beta_{2}\left[\tilde{\alpha}_{1}\left(5 \cos \left(\gamma_{1}\right) \cos ^{2}\left(\gamma_{2}\right)-\cos \left(\gamma_{1}\right)+2 \cos \left(\gamma_{2}\right) \cos (\tilde{\theta})\right)\right. \\
& \left.+\tilde{\alpha}_{2}\left(5 \cos \left(\gamma_{2}\right) \cos ^{2}\left(\gamma_{1}\right)-\cos \left(\gamma_{2}\right)+2 \cos \left(\gamma_{1}\right) \cos (\tilde{\theta})\right)\right], \\
& c_{4}^{(2)}=\frac{1}{7} \beta_{1} \beta_{2}\left[\frac{1}{5}+\frac{2}{5} \cos ^{2}(\tilde{\theta})-\left(\cos ^{2}\left(\gamma_{1}\right)+\cos ^{2}\left(\gamma_{2}\right)\right)\right. \\
& \left.+4 \cos \left(\gamma_{1}\right) \cos \left(\gamma_{2}\right) \cos (\tilde{\theta})+7 \cos ^{2}\left(\gamma_{1}\right) \cos ^{2}\left(\gamma_{2}\right)\right] \text {, }
\end{aligned}
$$


where the functions $\tilde{\alpha}_{i}$ were introduced as

$$
\tilde{\alpha}_{i}=\tilde{\alpha}_{i}\left(\chi_{i}, \chi\right):=\sinh (\chi) \alpha\left(\chi_{i}\right)=\sinh (\chi) \frac{\cosh \left(\chi_{i}\right)}{\sinh \left(\chi_{i}\right)}\left[2+\frac{\partial \ln \left(D_{i} f_{i} \Phi_{i}\right)}{\partial \ln \sinh \left(\chi_{i}\right)}\right], i \in\{1,2\} .
$$

\section{Flat FLRW Universe}

Consider now a flat universe. The two-point correlation function at present time is

$$
\xi(\chi)=\int_{0}^{\infty} \frac{\mathrm{d} \nu \nu^{2}}{2 \pi^{2}} X_{0}^{(0)}(\chi, \nu) S(\nu) ; \quad X_{0}^{(0)}(\chi, \nu)=\frac{\sin (\nu \chi)}{\nu \chi}=j_{0}(\nu \chi)
$$

where $S(\nu)$ is the total matter power spectrum and $j_{\ell}$ represents the spherical Bessel functions. On flat FLRW Universe models the eigenvalue of the Laplace operator $\nu=k$ is fully dimensional and so $S(\nu)=P(k)$, see $\S 2.1 .1$.

The two-point correlation function computation for flat Universes can be performed in a completely analogous way as for the open Universe case of the previous section. Alternatively, one can consider the flat limit for the open Universe results, i.e., the limit of curvature radius much larger than radial comoving distances $\left(K^{-1 / 2} \gg r\right)$, i.e., $\chi_{1,2}, \chi \rightarrow 0$ and $\nu \rightarrow \infty$ keeping $\chi \nu$ finite. Whichever approach is adopted, the main ingredients for the computation are the following: the well-known flat space version of the cosines law relating the radial separation between the objects $\chi$ with their radial positions and angular separation $\left(\chi_{1}, \chi_{2}, \theta\right)$,

$$
\chi^{2}=\chi_{1}^{2}+\chi_{2}^{2}-2 \chi_{1} \chi_{2} \cos (\theta)
$$

note that this is in fact the flat limit of equation (4.6), the angles between the lines of sight $\chi_{i}$ and the geodesic connecting the two points $\chi$, which are given by (see figure 4.1),

$$
\cos \left(\gamma_{1}\right)=\frac{\partial \chi}{\partial \chi_{1}}=\frac{\chi_{1}-\chi_{2} \cos (\theta)}{\chi}, \quad \cos \left(\gamma_{2}\right)=\frac{\partial \chi}{\partial \chi_{2}}=\frac{\chi_{2}-\chi_{1} \cos (\theta)}{\chi}
$$

they are also the flat limit of their open Universe versions, the variable $\tilde{\theta}$, defined in equation (4.9) for the open Universe case, which reduces in the flat limit to $\theta$, the angle subtended between the two object lines of sight.

Thus, the composition of redshift space operators acting on $X_{0}^{(0)}$ can be expanded as

$$
\left(\widehat{R}_{1} \circ \widehat{R}_{2}\right) X_{0}^{(0)}(\chi, \nu)=\sum_{n, \ell} c_{\ell}^{(n)}\left(\chi_{1}, \chi_{2}, \theta\right) \frac{(-1)^{n} X_{\ell}^{(0)}(\chi, \nu)}{\chi^{(2 n-\ell)} \nu^{2 n}}
$$

and consequently, the two-point correlation function in redshift space is given by equation (4.13) where the corresponding flat Universe multipoles are given by

$$
\Xi_{\ell}^{(n)}(\chi)=\frac{(-1)^{n}}{\chi^{2 n-\ell}} \int_{0}^{\infty} \frac{\mathrm{d} \nu \nu^{2}}{2 \pi^{2}} \frac{X_{\ell}^{(0)}(\chi, \nu)}{\nu^{2 n}} S(\nu)
$$

or, by using the relation $X_{\ell}^{(0)}(\chi, \nu)=(-1)^{\ell} \nu^{\ell} j_{\ell}(\nu \chi)$ (see Appendix B),

$$
\Xi_{\ell}^{(n)}(\chi)=\frac{(-1)^{n+\ell}}{\chi^{2 n-\ell}} \int_{0}^{\infty} \frac{\mathrm{d} k k^{2}}{2 \pi^{2}} P(k) \frac{j_{\ell}(k \chi)}{k^{2 n-\ell}}
$$


and the flat Universe coefficients $c_{\ell}^{(n)}$ are

$$
\begin{aligned}
c_{0}^{(0)=} & 1+\frac{1}{3}\left(\beta_{1}+\beta_{2}\right)+\frac{1}{15} \beta_{1} \beta_{2}\left(1+2 \cos ^{2}(\theta)\right) \\
c_{0}^{(1)}= & -\frac{1}{3} \beta_{1} \beta_{2} \tilde{\alpha}_{1} \tilde{\alpha}_{2} \cos (\theta) \\
c_{1}^{(1)}= & \beta_{1} \tilde{\alpha}_{1} \cos \left(\gamma_{1}\right)+\beta_{2} \tilde{\alpha}_{2} \cos \left(\gamma_{2}\right)+\frac{1}{5} \beta_{1} \beta_{2}\left[\tilde{\alpha}_{1}\left(\cos \left(\gamma_{1}\right)-2 \cos \left(\gamma_{2}\right) \cos (\theta)\right)\right. \\
& \left.+\tilde{\alpha}_{2}\left(\cos \left(\gamma_{2}\right)-2 \cos \left(\gamma_{1}\right) \cos (\theta)\right)\right] \\
c_{2}^{(1)}= & \beta_{1}\left(\cos \left(\gamma_{1}\right)-\frac{1}{3}\right)+\beta_{2}\left(\cos \left(\gamma_{2}\right)-\frac{1}{3}\right)-\frac{1}{7} \beta_{1} \beta_{2}\left[\frac{2}{3}+\frac{4}{3} \cos ^{2}(\theta)\right. \\
& -\left(\cos ^{2}\left(\gamma_{2}\right)+\cos ^{2}\left(\gamma_{2}\right)\right)+4 \cos \left(\gamma_{1}\right) \cos \left(\gamma_{2}\right) \cos (\theta) \\
& \left.+7 \cos ^{2}\left(\gamma_{1}\right) \cos ^{2}\left(\gamma_{2}\right)\right] \\
c_{0}^{(2)}= & 0, \\
c_{1}^{(2)=} & 0, \\
c_{2}^{(2)}= & \beta_{1} \beta_{2} \tilde{\alpha}_{1} \tilde{\alpha}_{2}\left(\cos \left(\gamma_{1}\right) \cos \left(\gamma_{2}+\frac{1}{3} \cos (\theta)\right)\right) \\
c_{3}^{(2)}= & \frac{1}{5} \beta_{1} \beta_{2}\left[\tilde{\alpha}_{1}\left(5 \cos \left(\gamma_{1}\right) \cos ^{2}\left(\gamma_{2}\right)-\cos \left(\gamma_{1}\right)+2 \cos \left(\gamma_{2}\right) \cos (\theta)\right)\right. \\
& \left.+\tilde{\alpha}_{2}\left(5 \cos \left(\gamma_{2}\right) \cos ^{2}\left(\gamma_{1}\right)-\cos \left(\gamma_{2}\right)+2 \cos ^{2}\left(\gamma_{1}\right) \cos (\theta)\right)\right] \\
& \frac{1}{7} \beta_{1} \beta_{2}\left[\frac{1}{5}+\frac{2}{5} \cos ^{2}(\theta)-\left(\cos ^{2}\left(\gamma_{1}\right)+\cos ^{2}\left(\gamma_{2}\right)\right)\right. \\
& \left.+4 \cos \left(\gamma_{1}\right) \cos \left(\gamma_{2}\right) \cos (\theta)+7 \cos ^{2}\left(\gamma_{1}\right) \cos ^{2}\left(\gamma_{2}\right)\right] \\
c_{4}^{(2)}= & \\
= & \\
= &
\end{aligned}
$$

where

$$
\tilde{\alpha}_{i}=\tilde{\alpha}_{i}\left(\chi_{i}, \chi\right):=\chi \alpha\left(\chi_{i}\right)=\frac{\chi}{\chi_{i}}\left[2+\frac{\partial \ln \left(D_{i} f_{i} \Phi_{i}\right)}{\partial \ln \left(\chi_{i}\right)}\right], \quad i \in\{1,2\}
$$

\section{Closed FLRW Universe}

Finally consider a closed universe $(K>0)$. The two-point correlation function of total matter at present time is given by

$$
\xi(\chi)=\sum_{\nu=3}^{\infty} \frac{\nu^{2}}{2 \pi^{2}} X_{0}^{(+)}(\chi, \nu) S(\nu) ; \quad X_{0}^{(+)}(\chi, \nu)=\frac{\sin (\nu \chi)}{\nu \sin (\chi)}
$$

where $S(\nu)$ is the adimensional total matter power spectrum related with its fully dimensional analog $P(k)$ according to equation (2.17), see $\S 2.1 .1$.

The two-point correlation function computation for closed Universes can be performed in a completely analogous way as for the open Universe case. Alternatively, one can consider the change of variables $\chi_{1,2} \rightarrow i \chi_{1,2}, \chi \rightarrow i \chi$ and $\nu \rightarrow-i \nu$ in the results obtained for open Universes. Whichever approach is adopted, the main ingredients for the computation are the following: the closed space version of the cosines law relating the radial separation between the objects $\chi$ with their radial positions and angular separation $\left(\chi_{1}, \chi_{2}, \theta\right)$,

$$
\cos (\chi)=\cos \left(\chi_{1}\right) \cos \left(\chi_{2}\right)-\sin \left(\chi_{1}\right) \sin \left(\chi_{2}\right) \cos (\theta)
$$


the angles between the lines of sight $\chi_{i}$ and the geodesic connecting the two points $\chi$, which are given by (see figure 4.1),

$$
\begin{aligned}
& \cos \left(\gamma_{1}\right)=\frac{\partial \chi}{\partial \chi_{1}}=\frac{1}{\sin (\chi)}\left[\sin \left(\chi_{1}\right) \cos \left(\chi_{2}\right)-\cos \left(\chi_{1}\right) \sin \left(\chi_{2}\right) \cos (\theta)\right] \\
& \cos \left(\gamma_{2}\right)=\frac{\partial \chi}{\partial \chi_{2}}=\frac{1}{\sin (\chi)}\left[\cos \left(\chi_{1}\right) \sin \left(\chi_{2}\right)-\sin \left(\chi_{1}\right) \cos \left(\chi_{2}\right) \cos (\theta)\right]
\end{aligned}
$$

and, in order to find the partial derivative of the $\gamma_{1,2}$ angles along the lines of sight $\chi_{i}$, the following identities hold [35]

$$
\begin{aligned}
\frac{\partial}{\partial \chi_{1}}\left(\sin \chi \cos \gamma_{1}\right) & =\cos \chi \\
\frac{\partial}{\partial \chi_{2}}\left(\sin \chi \cos \gamma_{1}\right) & =-\cos \chi \cos \tilde{\theta}
\end{aligned}
$$

which allow us to introduce the variable $\tilde{\theta}$ for closed Universes as

$$
\begin{aligned}
\cos (\tilde{\theta}) & =\frac{\sin \left(\gamma_{1}\right) \sin \left(\gamma_{2}\right)}{\cos (\chi)}-\cos \left(\gamma_{1}\right) \cos \left(\gamma_{2}\right)=\frac{\cos \left(\chi_{1}\right) \cos \left(\chi_{2}\right) \cos (\theta)-\sin \left(\chi_{1}\right) \sin \left(\chi_{2}\right)}{\cos \left(\chi_{1}\right) \cos \left(\chi_{2}\right)-\sin \left(\chi_{1}\right) \sin \left(\chi_{2}\right) \cos (\theta)} \\
& =\frac{\cos (\theta)-\tan \left(\chi_{1}\right) \tan \left(\chi_{2}\right)}{1-\tan \left(\chi_{1}\right) \tan \left(\chi_{2}\right) \cos (\theta)}
\end{aligned}
$$

Note that from this last relation, one can prove that for scales much smaller than the curvature scale, i.e., in the flat limit, $\chi_{1,2}=|K|^{1 / 2} x_{1,2} \ll 1, \tilde{\theta} \rightarrow \theta$. In fact, all the above equations in the flat limit reduce to the flat Universe ones of the previous section.

Thus, the composition of redshift space operators acting on $X^{(+)_{0}}$ can be expanded as

$$
\left(\widehat{R}_{1} \circ \widehat{R}_{2}\right) X_{0}^{(+)}(\chi, \nu)=\sum_{n, \ell} c_{\ell}^{(n)}\left(\chi_{1}, \chi_{2}, \theta\right) \frac{(-1)^{n} X_{\ell}(\chi, \nu)}{\sin ^{2 n-\ell}(\chi)\left(\nu^{2}+4\right)^{n}}
$$

and consequently, the two-point correlation function in redshift space is given by equation (4.13) where the corresponding closed Universe multipoles are given by

$$
\Xi_{\ell}^{(n)}(\chi)=\frac{(-1)^{n}}{\sin ^{2 n-\ell}(\chi)} \sum_{\nu=3}^{\infty} \frac{\nu^{2}}{2 \pi^{2}} \frac{X_{\ell}^{(+)}(\chi, \nu)}{\left(\nu^{2}+4\right)^{n}} S(\nu)
$$


and the closed Universe coefficients $c_{\ell}^{(n)}$ are given by

$$
\begin{aligned}
& c_{0}^{(0)}= 1+\frac{1}{3}\left(\beta_{1}+\beta_{2}\right)+\frac{1}{15} \beta_{1} \beta_{2}\left(1+2 \cos ^{2}(\tilde{\theta})\right) \\
& c_{0}^{(1)}=-\frac{1}{3} \beta_{1} \beta_{2} \tilde{\alpha}_{1} \tilde{\alpha}_{2} \cos (\tilde{\theta})+\left[\beta_{1}+\beta_{2}+\frac{2}{15} \beta_{1} \beta_{2}(4+3 \cos (\tilde{\theta}))\right] \sin ^{2}(\chi) \\
& c_{1}^{(1)}= \beta_{1} \tilde{\alpha}_{1} \cos \left(\gamma_{1}\right)+\beta_{2} \tilde{\alpha}_{2} \cos \left(\gamma_{2}\right)+\frac{1}{5} \beta_{1} \beta_{2}\left[\tilde{\alpha}_{1}\left(\cos \left(\gamma_{1}\right)-2 \cos \left(\gamma_{2}\right) \cos (\tilde{\theta})\right)\right. \\
&\left.+\tilde{\alpha}_{2}\left(\cos \left(\gamma_{2}\right)-2 \cos \left(\gamma_{1}\right) \cos (\tilde{\theta})\right)\right] \\
& c_{2}^{(1)}= \beta_{1}\left(\cos \left(\gamma_{1}\right)-1 / 3\right)+\beta_{2}\left(\cos \left(\gamma_{2}\right)-1 / 3\right) \\
&-\frac{1}{7} \beta_{1} \beta_{2}\left[2 / 3+4 / 3 \cos { }^{2}(\tilde{\theta})-\left(\cos ^{2}\left(\gamma_{2}\right)+\cos ^{2}\left(\gamma_{2}\right)\right)\right. \\
&\left.+4 \cos \left(\gamma_{1}\right) \cos \left(\gamma_{2}\right) \cos (\tilde{\theta})\right] \\
& c_{0}^{(2)}= \beta_{1} \beta_{2}\left(\sin ^{2}(\chi)-\tilde{\alpha}_{1} \tilde{\alpha}_{2}\right) \sin ^{2}(\chi) \\
& c_{1}^{(2)}= \beta_{1} \beta_{2}\left(\tilde{\alpha}_{1} \cos \left(\gamma_{1}\right)+\tilde{\alpha}_{2} \cos \left(\gamma_{2}\right)\right) \sin ^{2}(\chi) \\
& c_{2}^{(2)}= \beta_{1} \beta_{2} \tilde{\alpha}_{1} \tilde{\alpha}_{2}\left(\cos \left(\gamma_{1}\right) \cos \left(\gamma_{2}\right)+\frac{1}{3} \cos (\tilde{\theta})\right)+\frac{2}{7} \beta_{1} \beta_{2}\left[\cos { }^{2}(\tilde{\theta})-\frac{2}{3}\right. \\
&\left.+\frac{9}{2}\left(\cos ^{2}\left(\gamma_{1}\right)+\cos ^{2}\left(\gamma_{2}\right)\right)+10 \cos \left(\gamma_{1}\right) \cos \left(\gamma_{2}\right) \cos (\tilde{\theta})\right] \sin ^{2}(\chi) \\
& \frac{1}{5} \beta_{1} \beta_{2}\left[\tilde{\alpha}_{1}\left(5 \cos ^{(2)}\left(\gamma_{1}\right) \cos ^{2}\left(\gamma_{2}\right)-\cos \left(\gamma_{1}\right)+2 \cos \left(\gamma_{2}\right) \cos (\tilde{\theta})\right)\right. \\
&\left.+\tilde{\alpha}_{2}\left(5 \cos \left(\gamma_{2}\right) \cos ^{2}\left(\gamma_{1}\right)-\cos \left(\gamma_{2}\right)+2 \cos \left(\gamma_{1}\right) \cos (\tilde{\theta})\right)\right] \\
& c_{4}^{(2)}= \frac{1}{7} \beta_{1} \beta_{2}\left[\frac{1}{5}+\frac{2}{5} \cos ^{2}(\tilde{\theta})-\left(\gamma_{1}\right) \cos { }^{2}\left(\gamma_{2}\right)\right] \\
&\left.c_{3}^{(2)}\left(\gamma_{1}\right)+\cos { }^{2}\left(\gamma_{2}\right)\right)+4 \cos \left(\gamma_{1}\right) \cos \left(\gamma_{2}\right) \cos (\tilde{\theta}) \\
&+
\end{aligned}
$$

where

$$
\tilde{\alpha}_{i}=\tilde{\alpha}_{i}\left(\chi_{i}, \chi\right):=\sin (\chi) \alpha\left(\chi_{i}\right)=\sin (\chi) \frac{\cos \left(\chi_{i}\right)}{\sin \left(\chi_{i}\right)}\left[2+\frac{\partial \ln \left(D_{i} f_{i} \Phi_{i}\right)}{\partial \ln \sin \left(\chi_{i}\right)}\right], \quad i \in\{1,2\} .
$$

\section{Distant observer approximation}

In this section we recover the usual formula for the correlation function in redshift space from the general formulas obtained in the last sections. The limit to consider is the socalled distant observer approximation, which corresponds to the situation in which the distance between the two points is much smaller than the distances of the points to the observer, i.e. $\chi \ll \chi_{1}, \chi_{2}$. Formally, this limit should be taken maintaining the $\gamma_{1}$ and $\gamma_{2}$ angles fixed. In addition, one can consider that the scales of distance between the two objects is much smaller than the curvature scale, i.e., $\chi \ll 1$, a flat limit.

The first thing to note from this approximation is that, irrespective of the spatial curvature, it implies a small angle approximation in the sense that $\theta, \tilde{\theta} \rightarrow 0$. In fact, since one is considering scales between objects much smaller than the curvature scale, a flat limit applies even for the $K \neq 0$ cases. Thus, as discussed above, the variable $\tilde{\theta} \rightarrow \theta$. The fact that it goes to zero on the distant observer approximation follows from the flat cosines law, equation (4.18), as long as $\cos (\theta) \rightarrow 1$. Therefore, under the distant 
observer assumption, one is always treating points separated on the observer sky by a small angle. Usually separations below $10 \mathrm{deg}$ are considered in the literature, beyond which the deviation on the angle cosine with respect to one becomes larger than $2 \%$ for $z \sim 1$ [35]. However, the actual separation of the two points also depends on their redshifts and on the specific cosmological model.

Moreover, this small angle approximation also implies an identification of the angles $\gamma_{1}$ and $\gamma_{2}$. According to the flat Universe definitions of this angles, equations (4.19), follows that as long as $\theta \rightarrow 0, \cos \left(\gamma_{1}\right) \rightarrow-\cos \left(\gamma_{2}\right)$, and therefore $\gamma_{2} \rightarrow \pi-\gamma_{1}:=\gamma$. The interpretation of the angle $\gamma$ is that it naturally describes a single line of sight under small angle approximation. In other words, in the limit $\theta \rightarrow 0$, the two angles between the lines of sight to the objects $\chi_{1,2}$ and the geodesic connecting the objects $\chi$ are supplementary, so that define a unique line of sight. Thus, when one is working under the distant observer/small angle approximation one defines a unique line of sight for every pair of points, which can be one of the original line of sights or the bisector line in between. As long as the small angle approximation is valid, any of these choices should be equivalent.

Finally, consider the multipole expansion for the flat Universe redshift-space correlation function (equations (4.13) and (4.22)). Within the distant observer approximation, i.e. taking into account the above considerations, the $c_{\ell}^{(n)}$ coefficients reduce to

$$
\begin{aligned}
c_{0}^{(0)} & =1+\frac{1}{3}\left(\beta_{1}+\beta_{2}\right)+\frac{1}{5} \beta_{1} \beta_{2}, \\
c_{2}^{(1)} & =\left[\frac{2}{3}\left(\beta_{1}+\beta_{2}\right)+\frac{4}{7} \beta_{1} \beta_{2}\right] P_{2}(\cos (\gamma)), \\
c_{2}^{(4)} & =\frac{8}{35} \beta_{1} \beta_{2} P_{4}(\cos (\gamma)) .
\end{aligned}
$$

These coefficients are equivalent to those obtained in [33] under the additional assumption of the two points to be at the same redshift so that $\beta_{1}=\beta_{2}=\beta$. Thus, the two-point correlation function in redshift space considering the distant observer approximation can be written according to the expansion (2.19) as

$$
\begin{aligned}
\xi_{\mathrm{gg}}^{(\mathrm{rs})}(\chi)=\{1 & \left.+\frac{1}{3}\left[\beta_{\mathrm{g}}\left(z_{1}\right)+\beta_{\mathrm{g}}\left(z_{2}\right)\right]+\frac{1}{5} \beta_{\mathrm{g}}\left(z_{1}\right) \beta_{\mathrm{g}}\left(z_{2}\right)\right\} \xi_{0}(\chi) P_{0}(\cos (\gamma)) \\
& -\left\{\frac{2}{3}\left[\beta_{\mathrm{g}}\left(z_{1}\right)+\beta_{\mathrm{g}}\left(z_{2}\right)\right]+\frac{4}{7} \beta_{\mathrm{g}}\left(z_{1}\right) \beta_{\mathrm{g}}\left(z_{2}\right)\right\} \xi_{2}(\chi) P_{2}(\cos (\gamma)) \\
& +\left[\frac{8}{35} \beta_{\mathrm{g}}\left(z_{1}\right) \beta_{\mathrm{g}}\left(z_{2}\right)\right] \xi_{4}(r) P_{4}(\cos (\gamma))
\end{aligned}
$$

where $P_{\ell}(x)$ are the Legendre polynomials and the multipoles of the correlation function $\Xi_{\ell}^{(n)}$ (equation (4.21)) contributing must satisfy $2 n-\ell=0$ and $n+\ell$ even, so that they reduce to the distant observer two-point correlation multipoles,

$$
\xi_{\ell}(\chi):=\int_{0}^{\infty} \frac{\mathrm{d} k k^{2}}{2 \pi^{2}} j_{\ell}(k \chi) P(k)
$$

Note that the $\xi_{\ell=0}(\chi)$ is nothing but the two-point correlation function of total matter in real space (equation (2.21)). The angle $\gamma$ is determined for the unique line of sight 
(LOS) of the distant observer approximation. In this work this will be set as one of the two lines of sights, for example, taking the LOS as $\chi_{2}$ the angle $\gamma=\gamma_{2}$ so that

$$
\cos (\gamma)=\frac{\chi_{2}-\chi_{1} \cos (\theta)}{\chi}
$$

\subsubsection{Angular two-point correlation function of galaxies}

This section introduces the basic tool to study the angular clustering of galaxies, the angular two-point correlation function. Following the seminal works of $[7,8]$ one can begin by considering the projection of the spatial galaxy fluctuation today along a given direction in the sky (see $\S 1.4 .1$ for details),

$$
\delta_{\mathrm{g}}^{2 \mathrm{D}}\left(\mathbf{n}_{1}\right)=\int_{0}^{\infty} \mathrm{d} z W^{\mathrm{g}}(z) \widehat{R}_{\mathrm{g}} \delta_{0}\left(\chi, \mathbf{n}_{1}\right)
$$

here $\mathbf{n}_{1}=\left(\theta_{1}, \phi_{1}\right)$ denotes a given comoving angular position or, from the point of view of the observer, a direction in the sky, and $W^{\mathrm{g}}(z)=\phi^{\mathrm{g}}(z) b_{\mathrm{g}}(z) G(z)$ (equation (1.63)) is the galaxy window function, where $b_{g}(z)$ is a scale-independent galaxy bias, $G(z)$ the linear growth mode of matter fluctuations (see \$1.2.1) and $\phi^{\mathrm{g}}(z)$ the radial selection function of galaxies which allows us to introduce intrinsic characteristics of the observation, in particular it will be used to model the effect of photometric redshift errors (see §4.1.2).

The autocorrelation of $\delta_{\mathrm{g}}^{2 \mathrm{D}}$ as a function of the angular separation in the sky of two points define the angular correlation function of galaxies and can be obtained as the projection of the three dimensional correlation function along the lines of sight to the points as

$$
\begin{aligned}
w(\theta) & :=\left\langle\delta_{\mathrm{g}}^{(\mathrm{rs})}\left(\mathbf{n}_{1}\right) \delta_{\mathrm{g}}^{(\mathrm{rs})}\left(\mathbf{n}_{2}\right)\right\rangle=\int_{0}^{\infty} \mathrm{d} z_{1} \phi\left(z_{1}\right) \int_{0}^{\infty} \mathrm{d} z_{2} \phi\left(z_{2}\right)\left\langle\delta_{\mathrm{g}}^{(\mathrm{rs})}\left(\mathbf{n}_{1}, z_{1}\right) \delta_{\mathrm{g}}^{(\mathrm{rs})}\left(\mathbf{n}_{2}, z_{2}\right)\right\rangle \\
& =\int_{0}^{\infty} \mathrm{d} z_{1} \phi\left(z_{1}\right) \int_{0}^{\infty} \mathrm{d} z_{2} \phi\left(z_{2}\right) \xi_{\mathrm{gg}}^{(\mathrm{rs})}\left(z_{1}, z_{2}, \theta\right)
\end{aligned}
$$

here, $\theta$ is the angular separation between points in the sky, i.e., the angle subtended between the geodesics that connect the points with the observer (figure 4.1), and $z_{1,2}$ its respectives redshifts. Note that this formula is general in the sense that it is valid for any value for the separation of the galaxies and for any value of spatial curvature; the differences appear in the redshift space two-point correlation function $\xi_{\mathrm{gg}}^{(\mathrm{rs})}$ and were studied on $§ 4.1 .1$ in a completely general way, only assuming linear theory of cosmological perturbations. The general expression for $\xi_{\mathrm{gg}}^{(\mathrm{rs})}$, equation (4.1), allows for an expansion of the angular correlation function of galaxies of the form

$$
w(\theta)=\sum_{n, \ell} \int_{0}^{\infty} \mathrm{d} z_{1} W^{\mathrm{g}}\left(z_{1}\right) \int_{0}^{\infty} \mathrm{d} z_{2} W^{\mathrm{g}}\left(z_{2}\right) c_{\ell}^{(n)}\left(\chi_{1}, \chi_{2}, \theta\right) \Xi_{\ell}^{(n)}\left(\chi\left(\chi_{1}, \chi_{2}, \theta\right)\right) .
$$

Note that the formula (4.39) already includes the effects of spatial curvature, redshiftspace distortions and galaxy biasing for the angular correlation function. However, in the context of photometric galaxy surveys, two important physical effects still remain to be modeled, (a) the fact that the redshift of objects in the context of this astronomical surveys is not determined using accurate spectroscopic techniques but the techniques of photometry, i.e. by using information contained on the brightness of objects as viewed 
through various standard filters comprising a given broad spectrum of colors, which is useful in the context of estimating redshifts for a large number of objects; and (b) the nonlinear gravitational effects which are important at small scales, where the linear perturbation theory on top of the derivation of (4.39) should fail (see the discussion of $§ 1.2 .2$ for more details). In the following, the modeling of these two effects for the two-point correlation function of galaxies is considered.

\section{Photometric redshift estimates}

When an astronomical object is observed, its redshift (see $\S \mathrm{C} .1$ ) can be directly determined by an accurate measurement of its spectra, in which case absorption and emission lines can be used to estimate redshifts by comparing their wavelengths with the known (laboratory) wavelengths. Note that this interpretation relies on the assumption that atomic physics is unchanged over cosmological scales and also knowledge about galaxy evolution models. However, such a technique demands a great amount of time to collect spectra for each one of the objects in a survey. Thus, recent advances in galaxy surveys have been driven by new instrumentation that enables multiple galaxy spectra to be obtained simultaneously. It is remarkable the development of multi-object spectrographs (MOS) which enabled a number of survey teams to create maps of many hundreds of thousands or millions of galaxies. Examples of these surveys are the 2-degree Field Galaxy Redshift Survey (2dFGRS; [66]) and the Wigglez [67] surveys, that used the Anglo-Australian Telescope facilities, and the Sloan Digital Sky Survey (SDSS; [68]) conducted at the Sloan Telescope, specially designed for this propose. Typical errors for this kind of redshift estimates are $\sim 10^{-4}-10^{-3} \times(1+z)$.

However, recently, another alternative emerged with the possibility of fitting observed broad-band colors with templates or training samples of emitted light profiles of objects, and thus estimate their redshift. Such class of techniques are known as photometric redshifts, referring to redshifts estimated from broad-band colors only. An important characteristic of such estimates is that since they rely on known light profiles for specific classes of objects they vary in quality between galaxy samples, which leaves an accurate description of its errors as a very difficult task. In this sense photometric redshift estimates can have offsets from the true redshifts with standard deviations of $\sigma_{z} \sim 0.03(1+z)$ for red galaxies with strong $4000^{\circ} \mathrm{A}$ breaks, while more general populations can give estimates with $\sigma_{z} \sim 0.05(1+z)$ [69] or worse. In any case, these are much less accurate than the spectroscopic estimates. Nonetheless, the idea in the context of galaxy redshift surveys is that the estimates based on photometric techniques allow to simultaneously determine redshift of a great quantity of objects, so that in some sense one is exchanging the accuracy on individual redshifts for a better statistics in the context of clustering analysis. For a more detailed recent discussion see e.g. [70].

Photometric redshift uncertainties in our model for $w(\theta)$ (equations (4.38) and (4.39)) are included via the radial selection function of galaxies $\phi^{\mathrm{g}}(z)$. This function defines the probability to include a galaxy on a specific true redshift value $z$, so that, if the selection of galaxies is done according to their true redshifts, $\phi^{\mathrm{g}}(z)$ is simply the true number of galaxies $N_{\mathrm{g}}(z)$ per unit redshift, $n_{\mathrm{g}}(z)=\mathrm{d} N_{\mathrm{g}}(z) / \mathrm{d} z$, times a redshift (radial) window function, $W(z)$, encoding selection characteristics, e.g. redshift cuts in the observations and redshift binning.

$$
\phi^{\mathrm{g}}(z)=n_{g}(z) W(z)
$$

On other hand, if the selection is done according to photometric redshift estimates one 
can introduce the conditional probability $P\left(z \mid z_{\text {phot }}\right)$ for the true redshift to be $z$ when the photometric redshift (photo- $z$ ) is $z_{\text {phot }}$. Then the number density as a function of the true redshift should be properly given by the projection

$$
n_{\mathrm{g}}(z)=\int_{0}^{\infty} \mathrm{d} z_{\text {phot }} \frac{\mathrm{d} N_{\mathrm{g}}\left(z_{\text {phot }}\right)}{\mathrm{d} z_{\text {phot }}} P\left(z \mid z_{\text {phot }}\right) .
$$

In addition, a photometric redshift window function $W\left(z_{\text {phot }}\right)$ should also be projected to be a true redshift window function. Thus, we should have [71]

$$
\phi^{\mathrm{g}}(z)=n_{\mathrm{g}}(z) \int_{0}^{\infty} \mathrm{d} z_{\text {phot }} P\left(z \mid z_{\text {phot }}\right) W\left(z_{\text {phot }}\right) .
$$

Note that $\mathrm{d} N_{\mathrm{g}}\left(z_{\text {phot }}\right) / \mathrm{d} z_{\text {phot }}$, the number of galaxies per unit of photometric redshift can always be obtained by binning galaxies in a catalog in photo- $z$ and $W\left(z_{\text {phot }}\right)$ is given by the catalog characteristics. For example, when one is asking for the selection function on a given photo- $z$ bin, say the $i$-th one determined by photometric redshifts on the interval $\left[z_{\text {phot }}^{i}, z_{\text {phot }}^{i+1}\right]$, with selection $\phi_{i}^{\mathrm{g}}(z)$, one can write the photo- $z$ window as

$$
W^{i}\left(z_{\text {phot }}\right)= \begin{cases}1, & z_{\text {phot }}^{i} \leq z_{\text {phot }} \leq z_{\text {phot }}^{i+1} \\ 0, & \text { otherwise }\end{cases}
$$

In this way, the effects of photo- $z$ 's are then encoded on the conditional probability function $P\left(z \mid z_{\text {phot }}\right)$. Note that a perfect determination of redshifts can be parametrized by $P\left(z \mid z_{\text {phot }}\right)=\delta_{\mathrm{D}}\left(z-z_{\text {phot }}\right)$ and, in fact, under this assumption equation (4.42) reduces to (4.40). To go further, one can begin by a first idealized Gaussian approximation of the form

$$
P\left(z \mid z_{\text {phot }}\right)=\frac{1}{\sqrt{2 \pi} \sigma_{z}} \exp \left[-\frac{\left(z-z_{\text {phot }}-z_{\mathrm{b}}\right)^{2}}{2 \sigma_{z}^{2}}\right]
$$

where $\sigma_{z}$ and $z_{\mathrm{b}}$ represent the scatter and bias of a normal distribution and, in principle, can depend on redshift and object type.

The impact of these parameters is shown in figure 4.2. In order to properly account for only the photo- $z$ parameters the remaining set of cosmological parameters of a concordance cosmological model are chosen according to the WMAP7 results [72], see the discussion at the begining of paragraph 4.1.2 below. where it is shown that the scatter parameter $\sigma_{z}$ affects mainly the amplitude of the ACF leaving the position of the BAO peak unchanged, whereas the bias parameter $z_{\mathrm{b}}$ appears having a much more complex impact mixing the both, changes in the amplitude and in the position of the BAO peak. 

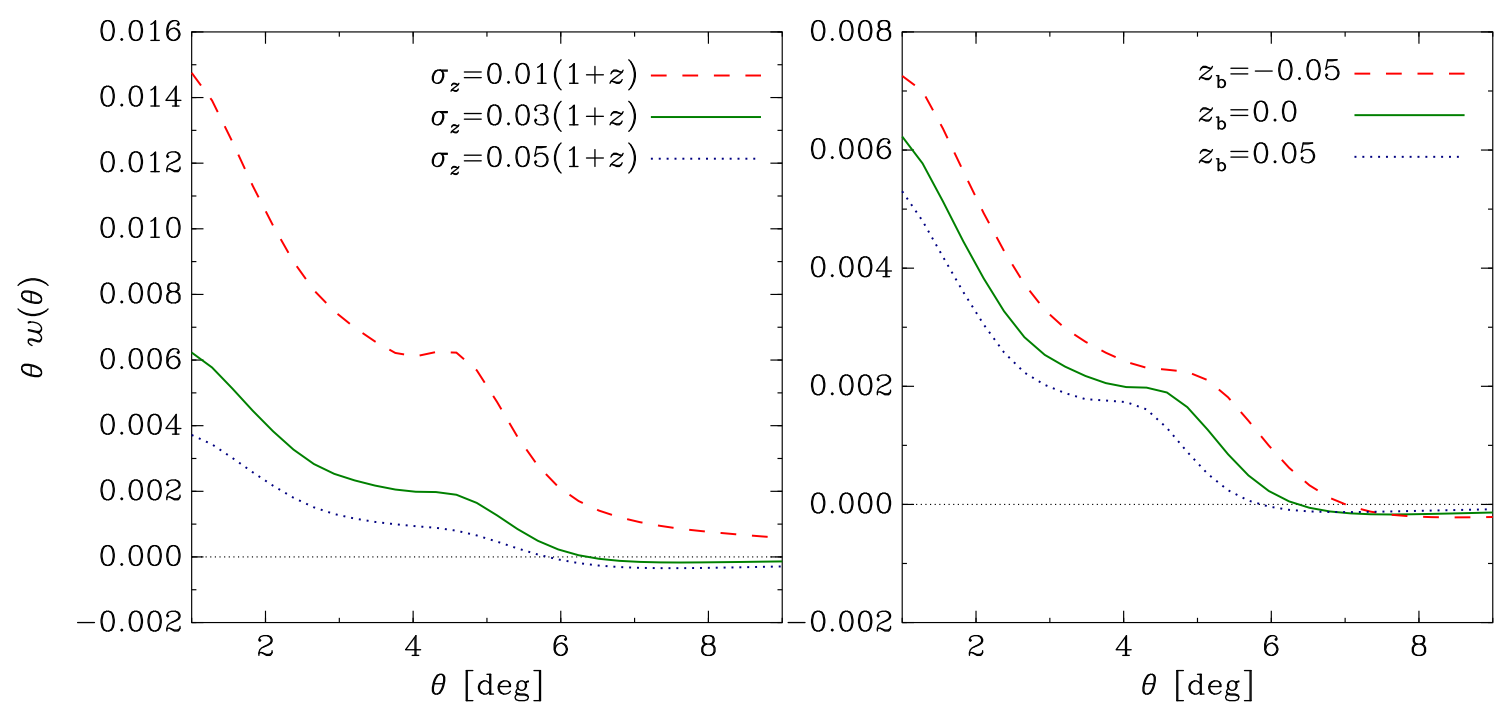

Figure 4.2: Impact of Gaussian photometric redshift errors (equation (4.44)) on the ACF. The left panel shows the impact of the scatter $\sigma_{z}$ whereas the right panel shows the impact of the bias $z_{\mathrm{b}}$. The major feature one can see is that the scatter does not have any impact on the position of the BAO angular scale, while the bias is inversely proportional to its value. The cosmological parameters assumed on these predictions are the WMAP7 results [72].

\section{Nonlinear gravitational effects}

The nonlinear (NL) gravitational evolution of matter fluctuations in the Universe becomes more important as one goes to smaller scales as discussed in section 1.2.2. To incorporate this effect on the modeling of the ACF in this work a Renormalized Perturbation Theory (RPT) phenomenological approach introduced by [30, 8] is adopted.

On $§ 1.2 .2$ a very brief discussion was made to introduce the full nonlinear power spectrum as expanded in RPT, equation (1.57). The configuration space analog of this expression was given in [30] to have the form

$$
\xi(r, z)=\left[G^{2} \otimes \xi_{0}\right](r, z)+\xi_{\mathrm{MC}}(r, z),
$$

where the symbol $\otimes$ indicates a convolution. Since in Fourier space the propagator $G$ is approximately Gaussian, the first term convolves the correlation function today, here denoted as $\xi_{0}$, with an approximately Gaussian kernel and $\xi_{\mathrm{MC}}$ account for the modecoupling term of the full NL power spectrum. Inspired on this particular form, [29, 8] proposed the following simple phenomenological parametrization

$$
\xi_{\mathrm{NL}}(r, z)=G^{2}(z) \int_{0}^{\infty} \mathrm{d} k P_{\mathrm{Lin}}(k) \exp \left[-\frac{r_{\mathrm{NL}}^{2} k^{2} G^{2}(z)}{2}\right]+A_{\mathrm{mc}} G^{4}(z) \xi_{\mathrm{Lin}}^{(1)}(r) \xi_{\mathrm{Lin}}^{\prime}(r),
$$

where

$$
\xi_{\text {Lin }}^{(1)}(r):=\frac{1}{2 \pi^{2}} \int_{0}^{\infty} \mathrm{d} k k P_{\text {Lin }}(k) j_{1}(k r)
$$

$G$ is the linear growth factor (not the propagator of RPT formalism anymore), $P_{\text {Lin }}$ refers to the linear power spectrum today and $r_{\mathrm{NL}}$ and $A_{\mathrm{mc}}$ can be seen as free parameters, shown in [8] to give good results by simple fixed to $r_{\mathrm{NL}} \approx 6.6 \mathrm{Mpc} h^{-1}$ and $A_{\mathrm{mc}} \approx 1.55$ for a large range of redshifts, up to $z \sim 1.3$ (the approximated depth of the DES) and on the scales of interest for clustering studies. 
Note then that the parametrization (4.46) allows for, given a linear power spectrum for a given set of cosmological parameters, properly correct for NL effects directly in configuration space. This is highly desirable in the context of ACF analysis and constitutes the main reason why we adopted here during the rest of this work.

\section{Cosmological information on the ACF}

The previous sections describe a complete model for the ACF on large scales, which take into account most of relevant effects, namely, nonlinear gravitational clustering, galaxy bias, redshift-space distortions and photo- $z$ uncertainties and assumes spatial flatness and the distant observer approximation in concordance with the seminal studies $[7,8]$. In this section, this model is now used to study the impact of different cosmological parameters on the ACF. Throughout this study, when not stated otherwise, a flat $\Lambda$ CDM Universe with parameters similar to those determined by WMAP7 [72] is assumed as fiducial cosmological model, i.e. the dark matter density parameter $\Omega_{\mathrm{c}}=0.222$, baryon density parameter $\Omega_{\mathrm{b}}=0.0449$, Hubble parameter $h=0.71$, primordial index of scalar perturbations $n_{s}=0.963$, and normalization of perturbations given by $\sigma_{8}=0.801$. The primordial power spectrum is assumed to be parametrized as usual in the literature, $P_{\text {prim }}(k)=A_{s}\left(k_{0}\right)\left(k / k_{0}\right)^{n_{s}-1}$, with the pivot scale $k_{0}=0.002 \mathrm{Mpc}^{-1}$, so only scalar perturbations are considered. Note that the $A_{s}$ parameter, the amplitude of primordial spectrum at the pivot scale, is directly related to $\sigma_{8}$, althought $\sigma_{8}$ can vary slightly with other parameters (e.g. $\Omega_{b}$ ). In the fiducial cosmology used here $A_{s}=2.142 \times 10^{-9}$. Nonlinear model parameters are assumed to be $r_{N L}=6.6 h^{-1} \mathrm{Mpc}$ and $A_{\mathrm{mc}}=1.55$, photometric redshift is assumed to be modeled by a Gaussian PDF for $P\left(z \mid z_{\text {phot }}\right)$ (equation (4.44)) with redshift scatter for a DES-like survey, $\sigma_{z}=0.03(1+z)$, and null bias, $z_{\mathrm{b}}=0.0$. The biasing effect for galaxies is assumed scale independent and constant for a sufficiently thin photometric redshift bin. The computations shown here are for a photometric redshift bin defined by $z_{\text {phot }} \in[0.45,0.50]$ with no other selection effect on photometric redshift than the binning, i.e., $W\left(z_{\text {phot }}\right)$ is given by equation (4.43). Thus the fiducial model assumes $b_{\mathrm{g}}=1$.

The impact of cosmological parameters on the ACF can be understood by coming from four different sources [7]: the total matter power-spectrum today $P(k)$, the growth function $G(z)$, the linear redshift-space distortion parameter $\beta_{g}(z)$, and the comoving distances. In general terms, the total matter power spectrum is characterized by the parameters $\Omega_{\mathrm{m}}, \Omega_{\mathrm{b}}, h, n_{s}$ and $\sigma_{8}$, The growth function as well as the RSD parameter are highly dependent on $\Omega_{\mathrm{m}}$, and comoving distances are determined by $\Omega_{\mathrm{m}}$ as long as they depend on the expansion history and, in this work, only a cosmological constant term is considered to drive cosmic acceleration.

In figure 4.3 it is shown how the ACF changes with relevant cosmological parameters. From the behavior of the ACF for the galaxy bias and $\sigma_{8}$, at the bottom of the figure, it is possible to appreciate the natural degeneracy between these two parameters. This degeneracy comes from the fact that both parameters enter the ACF as multiplicative factors for its amplitude on the same footing. Another important feature that can be seen from the figure is the displacement of the angular scale for the BAO peak. Among all the parameters considered just two of them appear to have a significant impact on this, namely, the Hubble factor $h$ and the content of baryons $\Omega_{\mathrm{b}}$. As it is well known the latter is strongly related with the BAO feature according to physics of the recombination era $[2,4]$. In fact, as can be seen from the figure, almost all the impact of $\Omega_{\mathrm{b}}$ on the ACF is on the BAO peak. On the other hand, the impact of $h$ on the angular scale of the BAO 
can be understood as coming from the radial distance dependence of the ACF.
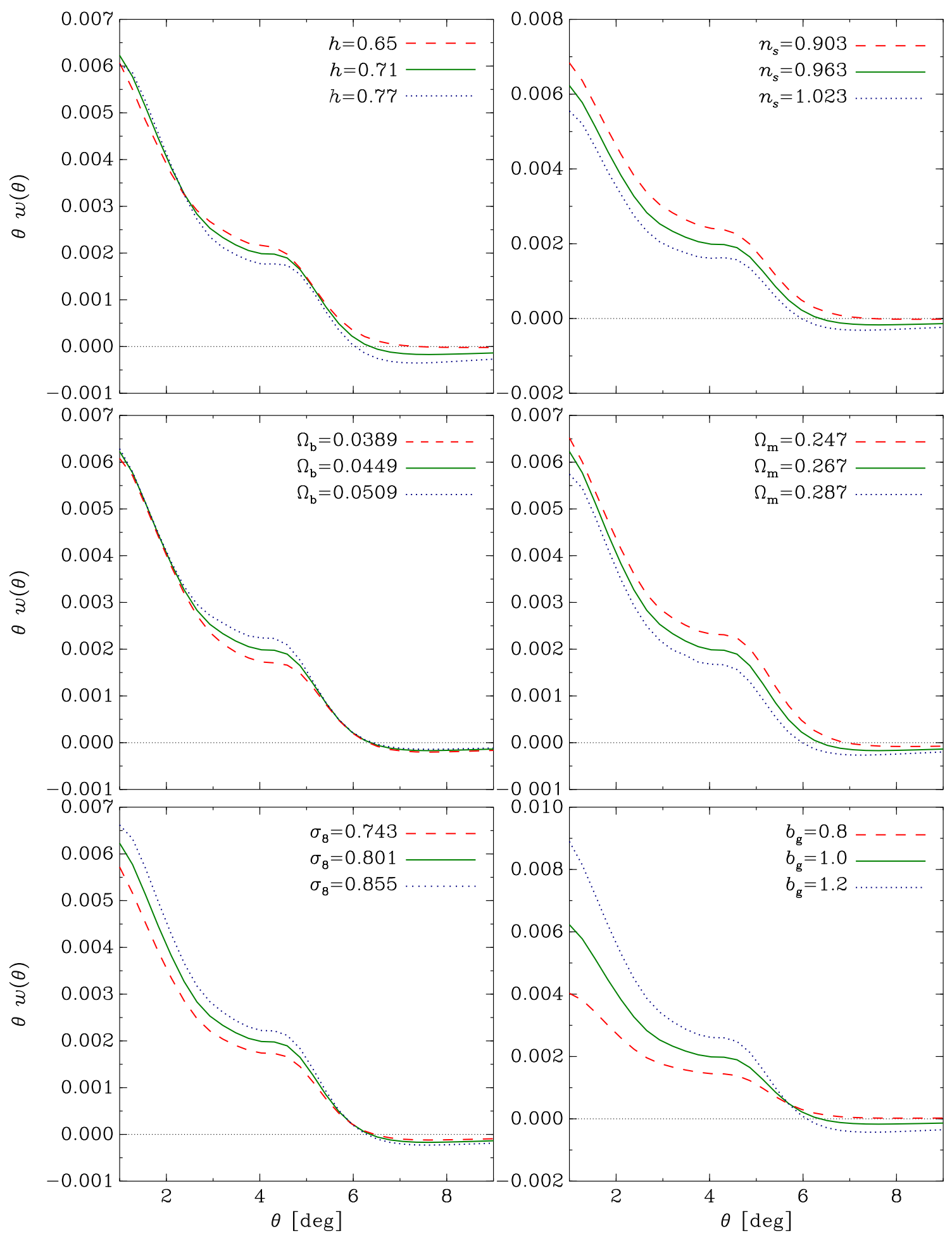

Figure 4.3: Impact of different cosmological parameters on the ACF. Each panel shows the the impact of one parameter on the ACF computed for a photometric redshift bin defined by $z_{\text {phot }} \in[0.45,0.50]$ with no selection effect on photometric redshift other than the binning. Photometric-z errors are assumed to be modeled by a Gaussian PDF for $P\left(z \mid z_{\text {phot }}\right)$ (equation (4.44)) with redshift scatter for a DES-like survey, $\sigma_{z}=0.03(1+z)$, and null bias, $z_{\mathrm{b}}=0.0$. Nonlinear model parameters are assumed to be fixed at $r_{N L}=$ $6.6 h^{-1} \mathrm{Mpc}$ and $A_{\mathrm{mc}}=1.55$. The fiducial cosmological model parameters shown in all panels as a green solid line are assumed to be the WMAP7 results [72]. 
The impact of the content of total matter and the spectral index of primordial fluctuations appear mostly on the amplitude of the ACF and can be understood as coming from the dependence of the total matter power spectrum today.

A complication in the simple effects illustrated above is the presence of photo- $z$ errors (figure 4.2), which can mimic some of the effects of changing the cosmological parameters in addition to redshift space distortions, nonlinearities and galaxy bias.

\section{Covariance of $w(\theta)$}

In configuration space clustering analysis, contrary to the situation in Fourier or harmonic space, the different scales in the correlation function are highly correlated, even in linear theory where the Fourier modes for the power spectrum can be considered as uncorrelated. As a result, the computation of the covariance of the correlation functions in real space is as important as the computation of the correlations themselves. In this section we review our method to compute the Gaussian theoretical covariance for the ACF including the effects of partial sky coverage, shot noise, photo- $z$ estimates and redshift-space distortions.

We follow the work of $[8,7]$ and take into account the correlation between redshift bins. Given two different redshift bins, namely $i$ and $j$, the full Gaussian covariance matrix can be computed as:

$$
\begin{aligned}
\operatorname{Cov}\left(w^{i}\left(\theta_{n}\right) w^{j}\left(\theta_{m}\right)\right) & :=\left\langle w^{i}\left(\theta_{n}\right) w^{j}\left(\theta_{m}\right)\right\rangle \\
& =\sum_{\ell, \ell^{\prime}=0}^{\infty} \operatorname{Cov}\left(C_{\ell}^{i} C_{\ell^{\prime}}^{j}\right) \frac{2 \ell+1}{4 \pi} \frac{2 \ell^{\prime}+1}{4 \pi} P_{\ell}\left(\cos \theta_{n}\right) P_{\ell^{\prime}}\left(\cos \theta_{m}\right),
\end{aligned}
$$

where the indexes $m, n$ denote angular bins and the $C_{\ell}$ represents the angular power spectrum modes of the ACF, see the $\S 2.2 .1$,

$$
w(\theta):=\sum_{\ell=0}^{\infty} C_{\ell} \frac{2 \ell+1}{4 \pi} \mathcal{P}_{\ell}(\cos (\theta)) .
$$

If our observations cover the full sky one can say that the $C_{\ell}$ 's are statistically independent. For partial sky coverage we can write (see e.g. the discussions in [8] and chapter 11 of [4])

$$
\operatorname{Cov}\left(C_{\ell}^{i} C_{\ell^{\prime}}^{j}\right)=\frac{2}{(2 \ell+1) f_{\text {sky }}}\left(C_{\ell}^{i, j}+\frac{\delta_{i j}}{\bar{n}_{i}}\right)^{2} \delta_{\ell \ell^{\prime}},
$$

which takes into accounts for a fraction of the sky $f_{\text {sky }}$ and also shot noise via $\bar{n}_{i}$ which defines the average number of galaxies per unit solid angle in the $i$-th $z$-bin. Note that shot noise between redshift shells are uncorrelated.

The effects of photo- $z$ and the redshift space distortion are included in the model for the angular power spectra of $w(\theta)$ following [8, 32]. Assuming a flat FLRW model and the validity of the distant observer approximation, the cross-correlation between $z$-bins can be computed as

$$
C_{\ell}^{i, j}=\frac{2}{\pi} \int \mathrm{d} k k^{2} P(k) \Psi_{\ell}^{i}(k) \Psi_{\ell}^{j}(k),
$$

where redshift-space distortions are included in the kernels,

$$
\begin{array}{r}
\Psi_{\ell}^{i}(k)=\int \mathrm{d} z \beta_{\mathrm{g}}(z) \phi_{i}^{\mathrm{g}}(z) G(z)\left[\frac{2 \ell^{2}+2 \ell-1}{(2 \ell+3)(2 \ell-1)} j_{\ell}(k r)-\frac{\ell(\ell-1)}{(2 \ell-1)(2 \ell+1)} j_{\ell-2}(k r)\right. \\
\left.-\frac{(\ell+1)(\ell+2)}{2 \ell+1)(2 \ell+3)} j_{\ell+2}(k r)\right] .
\end{array}
$$


Therefore, the general full covariance matrix that correlate angular and redshift bins is computed in this work as

$$
\operatorname{Cov}\left(w^{i}\left(\theta_{n}\right) w^{j}\left(\theta_{m}\right)\right)=\frac{2}{f_{\text {sky }}} \sum_{\ell=0}^{\infty}\left[\frac{2 \ell+1}{(4 \pi)^{2}} P_{\ell}\left(\cos \theta_{n}\right) P_{\ell}\left(\cos \theta_{m}\right)\left(C_{\ell}^{i, j}+\frac{\delta_{i j}}{\bar{n}_{i}}\right)^{2}\right] .
$$

\subsubsection{Computing the multipoles of the two-point correlation function}

The multipoles of the two-point correlation function appear in the theoretical modeling of the redshift-space correlation of tracers of the distribution of matter in the universe as discussed in section 4.1.1. In this section different approaches to the computation of the multipoles in the distant observer approximation (equation (4.35)) are considered. Hereinafter, the power spectrum of matter $P(k)$ is computed using the CAMB Boltzmann code [28].

\section{Integral method}

The most basic way to compute the $\xi_{\ell}$ is to perform directly the integration from its definition, equation (4.35). Note that in order to perform such an integration a numerical approximation scheme for the spherical Bessel functions $j_{\ell}$ is required. The Integral method developed in this work uses for the numerical integration an implementation of the Gauss-Kronrod method for unbounded integration interval [73] and the numerical approximation for the $j_{\ell}$ functions of the CAMB code [28].

\section{Hamilton method}

A second method follows from the fact that by using the recurrence relations of the spherical Bessel functions one can reduce the higher order multipoles of the correlation functions to integrals of its monopole, $\ell=0$, which corresponds to the total matter (realspace) two-point correlation function in real space for a spatially flat Universe [34]. For the cases of interest in equation (4.35) $\ell=2,4$ we have

$$
\begin{aligned}
& \xi_{2}(r)=\left[\frac{3}{r^{3}} \int_{0}^{r} \mathrm{~d} x \xi(r) x^{2}\right]-\xi(r), \\
& \xi_{4}(r)=\xi(r)+\frac{5}{2}\left[\frac{3}{r^{3}} \int_{0}^{r} \mathrm{~d} x \xi(x) x^{2}\right]-\frac{7}{2}\left[\frac{5}{r^{5}} \int_{0}^{r} \mathrm{~d} x \xi(x) x^{4}\right] .
\end{aligned}
$$

In order to prove these relations, consider the standard recurrence relations for the spherical Bessel functions and its derivatives [37]

$$
\begin{aligned}
j_{\ell-1}(x)+j_{\ell+1}(x) & =\frac{2 \ell+1}{x} j_{\ell}(x), \\
\frac{\mathrm{d}}{\mathrm{d} x}\left[x^{\ell+1} j_{\ell}(x)\right] & =x^{\ell+1} j_{\ell-1}(x), \\
\frac{\mathrm{d}}{\mathrm{d} x}\left[\frac{j_{\ell}(x)}{x^{\ell}}\right] & =-\frac{j_{\ell+1}}{x^{\ell}}
\end{aligned}
$$

Rewriting (4.55b) as

$$
j_{\ell}(r)=\frac{1}{r^{\ell+1}} \int_{0}^{r} \mathrm{~d} x x^{\ell+1} j_{\ell-1}(x)
$$


and noting that from $(4.55 \mathrm{a})$ one has

$$
\int_{0}^{\infty} \frac{\mathrm{d} k k^{2}}{2 \pi^{2}} j_{\ell+1}(k r) P(k)=\frac{2 \ell+1}{r} \int_{0}^{\infty} \frac{\mathrm{d} k k}{2 \pi^{2}} j_{\ell}(k r) P(k)-\int_{0}^{\infty} \frac{\mathrm{d} k k^{2}}{2 \pi^{2}} j_{\ell-1}(k r) P(k)
$$

one can write

$$
\begin{aligned}
\xi_{\ell+1}(r) & =\frac{2 \ell+1}{r} \int_{0}^{\infty} \frac{\mathrm{d} k k}{2 \pi^{2}} j_{\ell}(k r) P(k)-\xi_{\ell-1}(r) \\
& =\frac{2 \ell+1}{r} \int_{0}^{\infty} \frac{\mathrm{d} k k}{2 \pi^{2}}\left[\frac{1}{(k r)^{\ell+1}} \int_{0}^{k r} \mathrm{~d} y y^{\ell+1} j_{\ell-1}(y)\right] P(k)-\xi_{\ell-1}(r),
\end{aligned}
$$

introducing $y=: k x$ one finally gets

$$
\begin{aligned}
\xi_{\ell+1}(r) & =\frac{2 \ell+1}{r} \int_{0}^{\infty} \frac{\mathrm{d} k k}{2 \pi^{2}}\left[\frac{k}{r^{\ell+1}} \int_{0}^{r} \mathrm{~d} x x^{\ell+1} j_{\ell-1}(k x)\right] P(k)-\xi_{\ell-1}(r) \\
& =\frac{2 \ell+1}{r^{\ell+2}} \int_{0}^{r} \mathrm{~d} x x^{\ell+1} \int_{0}^{\infty} \frac{\mathrm{d} k k^{2}}{2 \pi^{2}} j_{\ell-1}(k x) P(k)-\xi_{\ell-1}(r)
\end{aligned}
$$

so that a general recurrence relation for the distant observer multipoles $\xi_{\ell}$ reads as

$$
\xi_{\ell+1}(r)=\frac{2 \ell+1}{r^{\ell+2}} \int_{0}^{r} \mathrm{~d} x x^{\ell+1} \xi_{\ell-1}(x)-\xi_{\ell-1}(r) .
$$

The equations (4.54) follow from this last expression for $\ell=1,3$ respectively.

The Hamilton method developed in this work implement the integrations of equations (4.54) by using a Gauss-Kronrod method [73] for a finite integration interval. The monopole computation on top of this integrations is done using the integral method of the previous paragraph with the analytic form of the $\ell=0$ spherical Bessel function, $j_{0}(z)=\sin (z) / z$. The advantage of Hamilton method of computation over the integral method is that one does not need to compute the spherical Bessel functions of higher order, which is computationally expensive.

\section{FFTLog method}

The last method considered uses the fact that the distant observer multipoles $\xi_{\ell}$ of the two-point correlation function and the power spectrum can be considered as a specific type of integral transform pair, namely, a Hankel transform [37].

Given an analytic function $a(r)$ defined on the interval $(0, \infty) \in \mathbb{R}$ a Hankel or FourierBessel transform pair is defined by the relations [37]

$$
\begin{aligned}
& \tilde{a}(k)=\int_{0}^{\infty} \mathrm{d} r k(k r)^{q} J_{\mu}(k r) a(r), \\
& a(r)=\int_{0}^{\infty} \mathrm{d} k r(k r)^{-q} J_{\mu}(k r) \tilde{a}(k),
\end{aligned}
$$

where the function $\tilde{a}$ is the Hankel transform of $a$. Note that this transformation depends on the values of $q$, the bias parameter and the order of the first kind Bessel function $\mu$. Under the changes of variables and $a(r)=: A(r) r^{-q}, \tilde{a}(k)=: \tilde{A}(k) r^{q}$ the Hankel transform pair can be rewritten as independent of the bias parameter,

$$
\begin{aligned}
& \tilde{A}(k)=\int_{0}^{\infty} \mathrm{d} r k J_{\mu}(k r) A(r), \\
& A(r)=\int_{0}^{\infty} \mathrm{d} k r J_{\mu}(k r) \tilde{A}(k) .
\end{aligned}
$$


Thus, the relation between the distant observer multipoles $\xi_{\ell}$ and the matter power spectrum $P(k)$ can be considered as a special case of unbiased Hankel transform pair. In fact, a more general relation can be found. Define the $\xi_{\ell}^{(m)}$ functions as

$$
\xi_{\ell}^{(m)}(r):=\frac{1}{2 \pi^{2}} \int_{0}^{\infty} \mathrm{d} k k^{m} j_{\ell}(k r) P(k) ; \quad \ell \in \mathbb{N},
$$

then, according to the relation between the spherical Bessel functions and the Bessel functions of the first kind [37], $j_{\ell}(z)=\sqrt{\pi / 2 z} J_{\ell+1 / 2}(z)$, one arrives to

$$
\xi_{\ell}^{(m)}(r)=\frac{1}{(2 \pi r)^{3 / 2}} \int_{0}^{\infty} \mathrm{d} k r J_{\ell+1 / 2}(k r)\left[k^{m-1 / 2} P(k)\right]
$$

so that the functions

$$
A(r)=(2 \pi r)^{3 / 2} \xi_{\ell}^{(m)}(r) \quad \text { and } \quad \tilde{A}(k)=k^{m-1 / 2} P(k)
$$

are related by an unbiased Hankel transform, equation (4.58), with $\mu=\ell+1 / 2$. Note that the distant observer multipoles, equation (4.35), of the two-point correlation function correspond to the case $m=2$.

In this sense, one translates the problem of computing the integrations considered in the Integral and Hamilton method discussed above to the computation of Hankel transforms. FFTLog is an algorithm designed to accomplish this goal by discretizing the functions, i.e., in an analogous way as the discrete Fourier transform for standard Fourier transformation problems [74]. The FFTlog method also takes into account an important feature for cosmological purposes. In cosmology one usually requires transforming a function that extends over many orders of magnitude, and was computed accurately in logarithmic space. For instance, that is the case of the matter power spectrum coming from Boltzmann codes, even in the linear regime, as the behavior of this function over at least 3 decades in $k\left(\sim 10^{-1}-10^{2} h / \mathrm{Mpc}\right)$ is required to properly compute the multipoles $\xi_{\ell}$ on large scales, see e.g. [64] for a discussion of this point. Besides, in practical applications as those discussed in this work, it is necessary to perform thousands or millions of such computations, so a fast transform method is highly desirable.

The FFTlog algorithm originally proposed in the cosmological context in [74] computes the fast Hankel (Fourier-Bessel) transform of a periodic sequence of logarithmically spaced points and thus can be regarded as a natural analogue to the standard Fast Fourier Transform (FFT) [75, 73], in the sense that, just as the usual FFT gives the exact (to machine precision) Fourier transform of a linearly spaced periodic sequence, representing the discretization of a function, so also FFTLog gives the exact Hankel transform of a logarithmically spaced periodic sequence, representing an appropriate discretization (for cosmological applications) of a function.

The FFTLog algorithm is reviewed in Appendix E. Its actual implementation for this work is a $\mathrm{C}++$ (object oriented) version of the original set of Fortran routines developed by A. Hamilton ${ }^{6}$.

\section{Comparison of the methods}

The methods for computing the distant observer multipoles of the two-point correlation function $\xi_{\ell}$ are compared in this section. The three methods defined on the last section

\footnotetext{
${ }^{6}$ http://casa. colorado.edu/ ajsh/FFTLog/
} 
are used to compute the $\ell=0,2,4$ distant observer multipoles (the ones entering into the computation of the distant observer two-point correlation function in redshift space, equation (4.34)) for the fiducial cosmological model described on paragraph 4.1.2 and on the physical scales relevant to clustering analysis $20<r<200 h^{-1} \mathrm{Mpc}$.

Our results are shown in figure 4.4 where the left panels show the monopole $\ell=0$ the middle panel the octupole $\ell=2$ and the right panels the hexadecapole $\ell=4$. For each one of these multipoles the absolute error of Hamilton and FFTLog methods with respect to the integral method are shown on the two bottom panels.
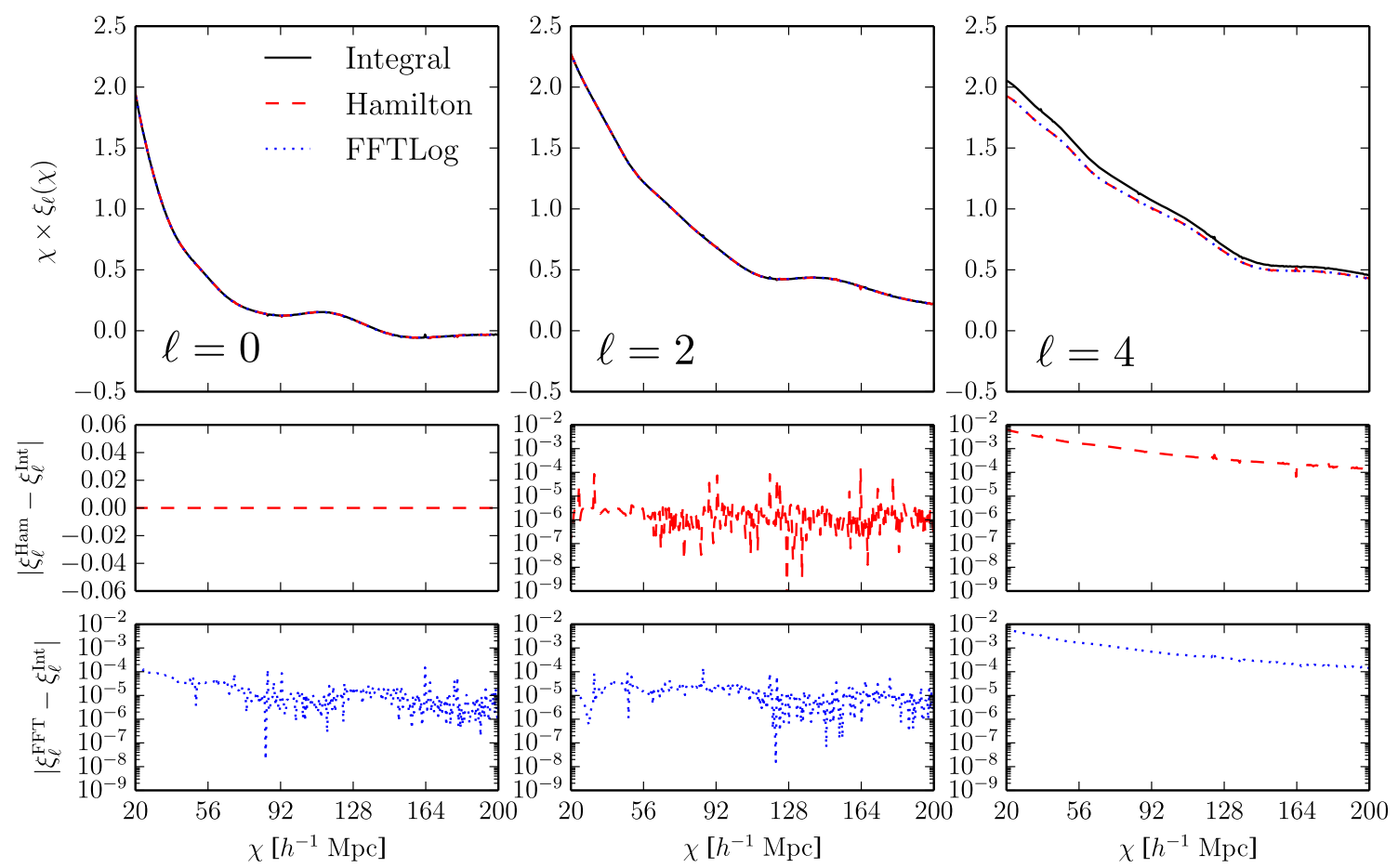

Figure 4.4: Comparison of the different methods presented to compute the distant observer multipoles of the two-point correlation function. The left panels show the monopole $\ell=0$, the middle panel the octupole $\ell=2$ and the right panels the hexadecapole $\ell=4$. For each one of these multipoles the absolute error of Hamilton and FFTLog methods with respect to the integral method is shown on the two companion bottom panels. The cosmological model used in the computation is given by the results of WMAP7 mission [72].

For all the multipoles, we found that the absolute difference between FFTLog and the Integral method is less than $10^{-2}$ as well as the absolute difference between the results of Hamilton method and Integral method. We found then that the results of FFTLog and Integral method are consistent with each other, even in a higher degree than with the Integral method for the highest multipole considered, the hexadecapole $(\ell=4)$, where the differences between FFTLog (Hamilton) method with respect to Integral can reach 1\% for small scales. We argue that this difference can be related with the numerical error in the computation of the spherical Bessel functions composed with the one comming from numerical integration.

We also compare the time required for each method to compute each one of the relevant distant observer multipoles on the same scales and for the same set of cosmological parameters. Our results are shown in table 4.1. 


\begin{tabular}{c|ccc}
\hline \hline $\begin{array}{c}\text { Multipole } \\
\text { order }\end{array}$ & \multicolumn{3}{|c}{ Method } \\
\hline$\ell=0$ & $21.13 \mathrm{sec}$. & $21.09 \mathrm{sec}$. & $2.00 \times 10^{-2}$ sec. \\
$\ell=2$ & $24.66 \mathrm{sec}$. & $19.64 \mathrm{sec}$. & $2.00 \times 10^{-2}$ sec. \\
$\ell=4$ & $25.81 \mathrm{sec}$. & $21.00 \mathrm{sec}$. & $2.00 \times 10^{-2}$ sec. \\
\hline \hline
\end{tabular}

Table 4.1: Comparison of the time required for the different methods presented for a single distant observer multipole computation. Each column shows the time in seconds required for a specific method to compute each one of the relevant multipoles, namely, the monopole $(\ell=0)$, the octupole $(\ell=2)$ and the hexadecapole $(\ell=4)$ The left panels show the monopole $\ell=0$ the middle panel the octupole $\ell=2$ and the right panels the hexadecapole $\ell=4$ on a single core processor of $2.4 \mathrm{GHz}$. The scales of the computation are $20<r<200 h^{-1} \mathrm{Mpc}$. The cosmological model used in the computation is given by the results of WMAP7 mission [72].

We found that the FFTLog method increase the computational speed in 2-3 orders of magnitude (in seconds) for all the relevant distant observer approximation multipoles and consequently in the computation of the distant observer approximation of the 3D and angular two-point correlation function in redshift space whithout a loss of accuracy when compared with the other methods. For this reason on all the ACF analysis presented in this work the FFTLog method is preferred to be used.

\subsubsection{SDSS-III DR8 photometric luminous galaxies ACF}

In the present section we present an analysis of the large scale angular correlation function (ACF) of the CMASS luminous galaxies (LGs), a photometric-redshift catalog based on the Data Release 8 (DR8) of the Sloan Digital Sky Survey-III. This catalogue contains over 600000 LGs in the range $0.45 \leq z \leq 0.65$, which was split into four redshift shells of constant width. Our analysis shows that the ACF can be efficiently applied to constrain cosmology in future photometric galaxy surveys.

Photometric galaxy surveys will demand a full understanding of the angular clustering of the galaxy distribution in order to provide useful cosmological information. Consequently, several studies have been performed in order to gauge the use of the galaxy angular clustering at large scales, both on theoretical and observational grounds. Let us begin by briefly review some of them below.

\section{Clustering in SDSS}

On the theoretical front, [76] performed the first study on the measurement of the baryon acoustic oscillation (BAO) peak in the galaxy angular correlation function (ACF) in configuration space using photometric redshifts. They emphasized the role of photo- $z$ errors in establishing the connection between the observed BAO position and the sound horizon scale. [7] forecasted the cosmological constraints in a DES-like galaxy survey from the $\mathrm{ACF}$ full shape information using the Fisher matrix formalism. They found that DES will constrain the dark energy equation of state parameter $w$ with a precision of $\sim 20 \%$. [8] verified the accuracy of the ACF theoretical covariance matrix against $N$-body simulations, showing that, at scales larger than $\sim 20 h^{-1} \mathrm{Mpc}$, the Gaussian covariance is a good approximation. [69] forecasted constraints on redshift-space distortion (RSD) parameters for a DES-like survey from the ACF full shape information and [77] developed a method 
to apply the BAO peak position in the $\mathrm{ACF}$ as a standard ruler, overcoming some issues outlined in [76].

On the observational front, only one galaxy survey had the characteristics to make it possible to look into the large scale properties of the ACF using photo-zs: the SDSS. This survey produced a series of data releases with four of them leading to a cosmological analysis with photometric data: Data Release 3 [78]; the DR4 which was used to produce the MegaZ photometric catalogue [79], the DR7 [80] and the recent DR8 luminous galaxies (LGs) catalogue [69]. These four photometric catalogues resulted in a series of results on the angular clustering of galaxies at large scales, mostly in the redshift range $0.45 \leq z \leq$ 0.65 .

[32] estimated the angular power spectrum in eight redshift shells, constraining RSD parameters and $\Omega_{\mathrm{m}}$. [81] used the MegaZ catalogue to produce the first cosmological constraints directly from the galaxy angular clustering using the angular power spectrum. [82] measured the large scale ACF, but did not constrain cosmological parameters due to an excess power at these scales. [83] produced a similar analysis as that of [81], but for the improved DR7. [8] used DR7 data to constrain the so-called RSD parameters with the ACF full shape information, but did not estimate the cosmology. [84] used the BAO peak position information in DR7 to find the sound horizon scale. [69] measured the large scale ACF in DR8 in order to check the impact of systematics, reducing the excess of power at these scales reported earlier [82, 85]. Using the DR8, [86] estimated the cosmological parameters from the full information of the angular power spectrum and [87] found the sound horizon scale also from the angular power spectrum. It is important to note that the cosmological analysis in all of these studies was performed in harmonic space with the angular power spectrum, not in configuration space with the ACF full shape information.

In our work we focus on the less explored approach of using the full shape of the $\mathrm{ACF}$ in configuration space to derive constraints on cosmological parameters, following the steps outlined in [7]. For these purposes we measure the ACF with the SDSS-III DR8 photometric data, using the so-called CMASS LGs catalogue [69]

\section{The catalog}

We use the imaging data from the SDSS-III DR8 [88], which is publicly available by the SDSS team ${ }^{7}$. The total sky coverage of imaging data in DR8 is summarized on top-left panel of figure 4.5 It had been calculated more carefully than DR7, covering a total unique imaging area of $14.555 \mathrm{deg}^{2}$, with a new area of $\sim 2.500 \mathrm{deg}^{2}$ since DR7 and containing 469.053.874 objects in database [88]. This survey obtained wide-field CCD photometry in five passbands: $u, g, r, i, z$

In order to obtain the spectroscopic redshifts, $z_{\text {spec }}$, it was used spectroscopic data from the SDSS-III Baryon Oscillation Spectroscopic Survey (BOSS) [89]. BOSS is a spectroscopic survey running from Fall 2009 up to Summer 2014 over a sky coverage of $10.000 \mathrm{deg}^{2}$ depicted on top-right panel of figure 4.5. It will target $1.5 \times 10^{6}$ massive galaxies $(z<0.7, i<19.9), 1.5 \times 10^{5}$ quasars $(z \geq 2.2$ and $g<22.0)$ selected from $4 \times 10^{5}$ candidates, and 75,000 ancillary targets [89]

\footnotetext{
${ }^{7}$ The data can be downloaded at http://portal.nersc.gov/project/boss/galaxy/photoz/
} 

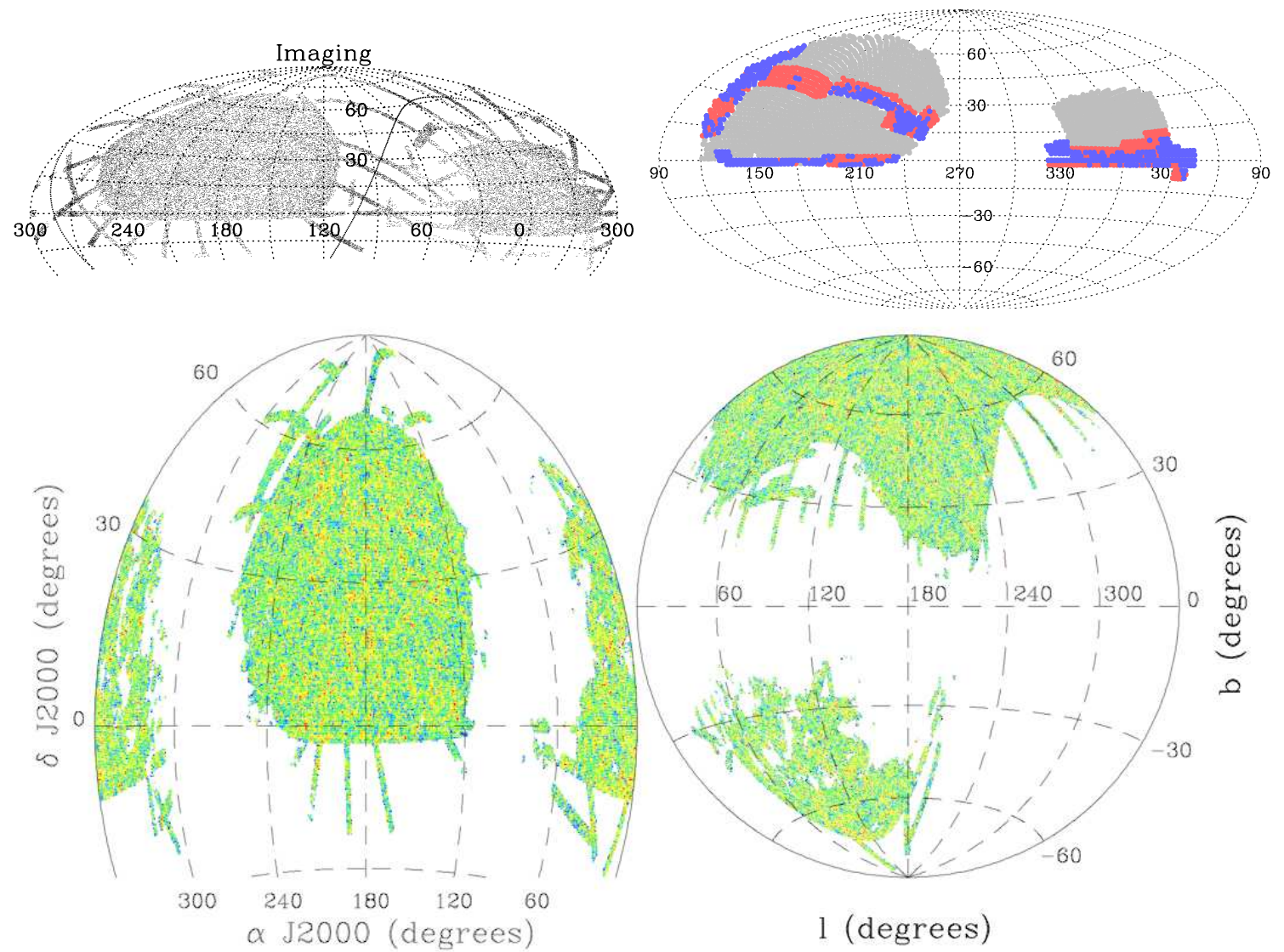

Figure 4.5: SDSS-III DR8 LG's catalog. Top-left panel: sky coverage in imaging data. The contiguous imaging coverage of the Southern Galactic Cap (centered roughly at $\alpha=0^{\circ}, \delta=+10^{\circ}$ ). The Galactic plane is the solid curve that snakes through the figures. Right ascension $\alpha=120^{\circ}$ is shown at the center of the plots. Figure from [88]. Top-right panel: planned footprint of the BOSS spectroscopic survey, showing both the NGC (left) and SGC (right) regions. Each circle marks the location of a spectroscopic plate. Blue circles represent plates that have been observed as of January 2011, while red circles represent plates that have been drilled but not yet observed. Figure from [89]. Bottom panels: resulting density of objects in the LG's catalog, in equatorial coordinates (left panel) and Galactic coordinates (right panel), after masking for imaging area, seeing, Galactic extinction, and bright stars. This masked footprint occupies $9.913 \mathrm{deg}^{2}$. The density increases from blue to red, with blue representing a density that is less than $40 \%$ of the average and red representing a density that is $120 \%$ greater than average. Figure from [69].

The construction of this photometric catalogue is detailed in [69, 90], where special care was taken to identify and remove potential systematic errors that could affect the measurement of the angular clustering of galaxies. The photometric catalog has the same selection as the sample of BOSS targets chosen to have approximately constant stellar mass, denoted "CMASS" as described by [89] and reviewed in [69]. The target selection criteria produce a sample of over $1.6 \times 10^{6}$ objects over a sky coverage $\sim 11.000 \mathrm{deg}^{2}$. Following [69], we will call this objects as Luminous Galaxies (LG). The sample was cut further down to the main SDSS imaging area. This area is defined as the data contained in HEALPix [52] pixels with $N_{\text {side }}=1024$, this choose of the resolution breaks down the full sky into 12,582,912 equal-area pixels. Each one of this pixels is endowed with a weight given its overlap with the imaging footprint (properly accounting for the area 
taken up by bright stars, stellar masking), and there was included only pixels with the this weight $>0.9$.

With the appropriate selection and cuts, one ends up with a LG's catalogue containing $\sim 7.0 \times 10^{5}$ galaxies, mostly in the photometric redshift range $z_{\text {phot }} \in[0.45,0.65]$, which is going to be our limiting redshifts for the cosmological analysis. We split the catalog into 4 photo- $z$ bins of width $\Delta z_{\text {phot }}=0.05$ and use measurements of the ACF for each $z$-bin.

The true redshift distribution of tracers is one of the most important and challenging quantities needed in order to produce results when investigating the projected angular clustering within a redshift bin. For a cosmological analysis it is as important as the ACF measurement itself. For the LG's sample used in this work, the photo- $z$ 's of the objects are fairly accurate. They were estimated with the neural network ANNz code [91] using as training set 112,778 spectra, i.e. almost $10 \%$ of the final photometric LG's sample. The photo- $z$ dispersion and the number of galaxies in each of the four $z$-bins used in this work are displayed in table 4.2 .

\begin{tabular}{c|ccc}
\hline \hline$z$-bin & photo- $z$ range & Number of galaxies & $\sigma_{\mathrm{ph}}$ \\
\hline 1 & {$[0.45,0.50]$} & 154531 & 0.043 \\
2 & {$[0.50,0.55]$} & 198132 & 0.044 \\
3 & {$[0.55,0.60]$} & 190603 & 0.052 \\
4 & {$[0.60,0.65]$} & 121181 & 0.063 \\
\hline \hline
\end{tabular}

Table 4.2: Redshift bins used in the analysis of SDSS-III DR8 catalog. Columns show, for each bin, the photo- $z$ range, the number of galaxies from [86] and the mean photo- $z$ dispersion from [69].

The selection function convolves the redshift distribution with the photo- $z$ errors and must be included in the ACF calculation as described in $\S 4.1 .2$. In figure 4.6 we show the selection functions for the four redshift bins as estimated by [69], which is also publicly available. Note that, as expected, the selection functions overlap due to photo- $z$ uncertainties. In this work we properly account for this effect both in the ACF itself and its covariance matrix, which accounts for the correlation amongst redshift and angular bins.

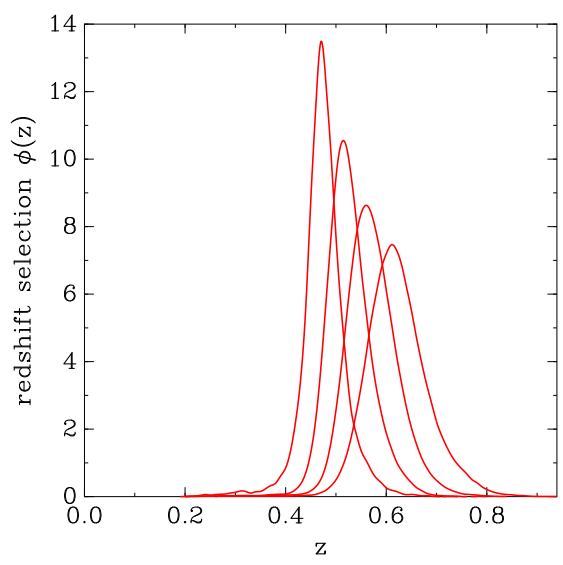

Figure 4.6: Radial selection functions for the different photo- $z$ bins considered in the SDSS-III DR8 analysis [69]. The details of the bins can be found on Table 4.2. 


\section{Measuring and modeling the SDSS-III DR8 LG's ACF}

In this work the ACF has been measured in 35 angular bins of width $\Delta \theta=0.2$ deg in the range $1<\theta<8$ deg for all the photo- $z$ bins. Such intervals were chosen in order to approximately guarantee that the analysis is done on large scales, i.e. for comoving distances in the range $20<r<200 h^{-2} \mathrm{Mpc}$ on all photo- $z$ bins. This a basic requirement in order to use our model for the covariance of the ACF, since it comes from the assumption of Gaussianty at large scales [8], see 4.1.2.

This measurements were performed following [69] and [86]. This approach has the tremendous advantage of incorporate systematics effects, such as spurious clustering power due to extinction, seeing and star contaminations. In the present work we do not pretend to explain in detail how this is done, however, in the following we review this systematic effects and the practical implementation of the algorithm of estimation. The interested reader can see the original references $[69,86]$.

We can begin by talking about the effect of stellar contamination in a galaxy sample, it is well documented on literatire, see e.g. [92]. Stellar contamination is used to denote the fact that stars may cause a systematic effect on the number density of objects by occulting a small fraction of the sky. This area is on the order of $10^{-6} \mathrm{deg}^{2}$ per star, but with $\sim 10^{4}$ (tens of millions) of stars, becomes substantial given the precision to which clustering measurements can now be made.

Following one has Galactic extinction, it requires that magnitudes be corrected for the effect of dust in our Galaxy. errors in this correction may cause a systematic effect on the galaxy density field, as the effective depth of a survey would fluctuate. Further, The expected magnitude error will vary as a function of the Galactic extinction. This is commonly understood as follows: constant (extinction corrected) magnitudes have different fluxes (since the flux is directly related to the magnitude before extinction corrections).

Other further effect is Airmass which refers to the path length of the photons through our atmosphere to the telescope, normalized to unity for observations at the zenith where it is minimized. At higher airmass less photons reach the detector because more are scattered/absorbed in the atmosphere and thus the error on a measured magnitude will be related to the airmass. It has then similar consequences to galactic extinction effect.

Finally, it is considered seeing effect. The observed flux of an object is more spread out at higher seeing. This implies an increase in the magnitude error and makes it more difficult to distinguish between stars and galaxies. Either of these seeing-dependent effects could cause spurious fluctuations in the observed density of galaxies and was consider in detail on [69].

Coming back to the measurements of the ACF, the catalog was pixelized at $N_{\text {side }}=256$ using HEALPix [52] and each pixel $i$ is assigned a weight $w t_{i}$ related to its overlap with the imaging footprint, and allowing for the account of the systematic effects discussed above [69]. The estimated ACF $\hat{w}(\theta)$ is obtained as

$$
\hat{w}(\theta)=\frac{\sum_{i j} \delta_{i} \delta_{j} w t_{i} w t_{j}}{\sum_{i j} w t_{i} w t_{j}}
$$

where $\theta$ is the angular distance between pixel $i$ and pixel $j$ and the overdensity in pixel $i$, $\delta_{i}$, is given by

$$
\delta_{i}=\frac{n_{i}}{\bar{n} w t_{i}}-1
$$

with $n_{i}$ is the number of galaxies in pixel $i$ and $\bar{n}=\sum n_{i} / \sum w t_{i}$. 
The results for the ACF in each photo- $z$ bin is shown in black in figure 4.10. They do not show the excess of power at large scales found previously by [83] and [82], also remarkably is the fact that the BAO peak can be distinguished.

The model for the ACF used during this analysis is the one described in section 4.1.2, i.e. the distant observer approximation is assumed along with the spatial flatness of the FLRW cosmological model used to describe the observations. The former assumption is supported by the facts that we are measuring the ACF on small angular scales and the redshifts of LGs are small. By the other hand, the latter assumption has no other justification from simplicity on the analysis. Sometimes in the literature the phrase inflation predicts $\Omega_{K}=0$ is found, however, that is not true, what inflation predicts is $\Omega_{K} \rightarrow 0$. This discussion may seems academic, but it has far-reaching implications, for example, $K>0$ implies that the background FLRW Universe has closed constant time hypersurfaces, so it is finite (see section 1.1), besides, the two-point statistics on such models now at linear perturbative level has interesting properties as long as the spectrum of the spatial Laplace operator on the constant time hypersurfaces is discrete (see discussion in Appendix B and chapter 2). All the relevant effects were taken into account, photo- $z$ uncertainties, according to the discussion in the previous paragraph, nonlinearities in the clustering of galaxies, redshift-space distortions and galaxy biasing. For the nonlinearities it was assumed $r_{\mathrm{NL}}=6.6 \mathrm{~h}^{-1} \mathrm{Mpc}$ and $A_{\mathrm{mc}}=1.5$ in the RPT parametrization. The galaxy biasing effect (see the begining of $\S 1.4 .1$ for some discussion) was modeled assumed to be constant on each photo- $z$ bin. Thus hereinafter the bias of LGs for each of the photo- $z$ bins in the SDSS-III DR8 catalog described in table 4.2 are parametrized as $b_{1}, b_{2}, b_{3}$ and $b_{4}$ in the order of increasing photometric redshift.

It is well known that the estimation of the covariance matrix for a galaxy clustering analysis in configuration space is both a fundamental but a very difficult task. The standard way to construct the covariance matrix $C\left(\theta_{i}, \theta_{j}\right)$, between angular bins $i$ and $j$, is by the use of bootstrap methods, i.e., applying the data itself in the estimation. The most widely used approach is the so-called jack-knife method, see e.g. [69]. However, in order to perform the cosmological analysis, we modeled theoretically the ACF covariance matrix according to section 4.1.2, i.e. assuming Gaussianity at large scales as given by equation (4.53). This approximation is in very good agreement with the covariance obtained from N-body simulations, see e.g. [8]. Note that our model for the ACF covariance properly account for partial sky coverage and shot noise as well as for the same effects considered for the ACF modeling, namely RSD, biasing, nonlinearities and the photo- $z$ effects. Nevertheless, we do not allow for a cosmology-dependent covariance analysis because of computational time reasons, so we need to fix the cosmological parameters to a fiducial model and choose an appropriate value for the galaxy bias according to the galaxy sample. We assume as fiducial cosmological model a flat $\Lambda$ cold dark matter $(\Lambda \mathrm{CDM})$ Universe with parameters as determined by WMAP7 [72]: dark matter density parameter $\Omega_{\mathrm{c}}=0.222$, baryon density parameter $\Omega_{\mathrm{b}}=0.0449$, Hubble parameter $h=0.71$, primordial index of scalar perturbations $n_{s}=0.963$, and normalization of perturbations $\sigma_{8}=0.801$. We also fix nonlinear RPT parametrization parameters to $r_{\mathrm{NL}}=6.6 h^{-1} \mathrm{Mpc}$ and $A_{\mathrm{mc}}=1.55$ as for the ACF. The galaxy bias is also assumed to be constant on each photo- $z$ bin. A natural problem arising from this approach is the determination of the values for the LGs bias on each redshift bin to use in the covariance matrix. In order to solve this problem the following strategy was adopted. First cosmology was fixed, in this case the WMAP7 just presented, and an initial constant value $b=2$ for the bias was assigned on each photo- $z$ bin, from which a covariance matrix was generated. Next, the best-fit values for 
the bias was determined using the ACF full shape information as will be explained in the following section. In our case the following results were encountered $b_{1}=1.94, b_{2}=2.02$, $b_{3}=2.15$, and $b_{3}=1.97$. With the ingredients just listed above the resulting theoretical covariance matrix used on our analysis is shown on figure 4.7.

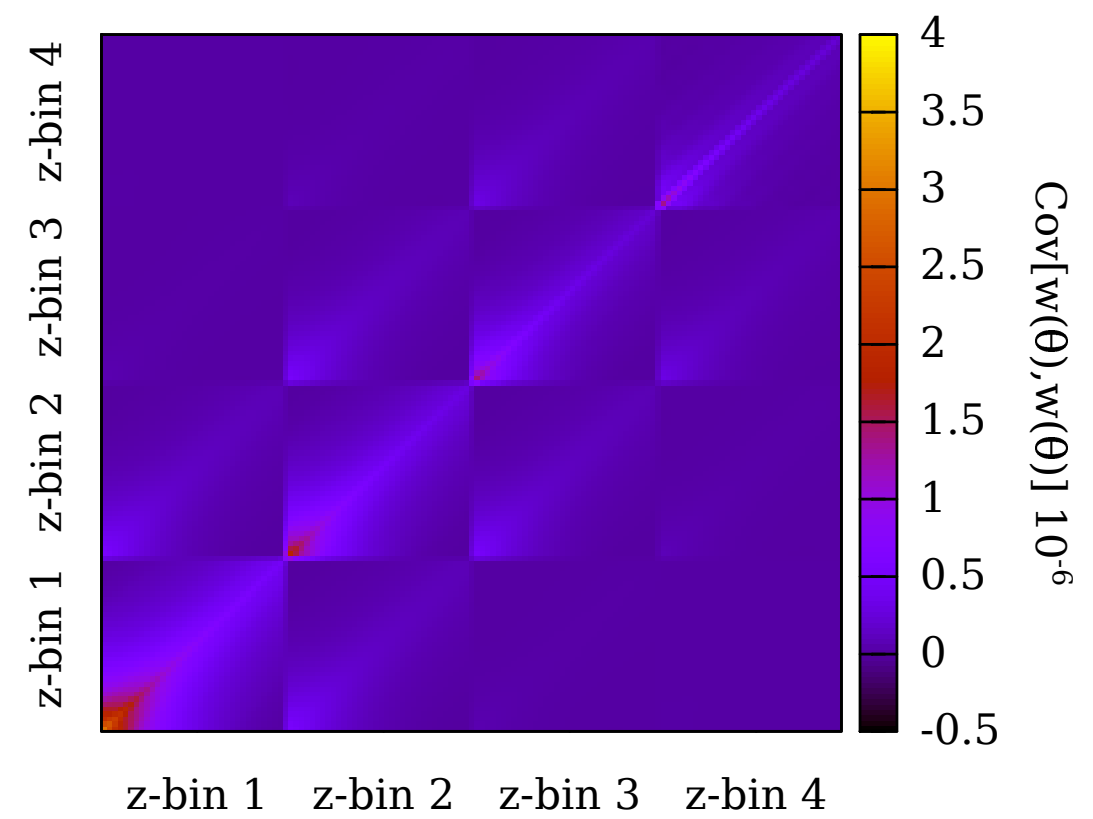

Figure 4.7: Theoretical ACF covariance matrix used in the SDSS-III DR8 analysis. Since we do not allow for an analysis with a cosmology-dependent theoretical covariance because of computational time reasons, we fix the theoretical covariance to a flat FLRW model with parameters $\Omega_{\mathrm{c}}=0.222, \Omega_{\mathrm{b}}=0.0449, h=0.71, n_{s}=0.963$, and $\sigma_{8}=0.801$. The galaxy bias is assumed to be constant on each of the photo- $z$ bins (table 4.2) and given by $b_{1}=1.94, b_{2}=2.02, b_{3}=2.15$, and $b_{3}=1.97$. Also nonlinearities are fixed according to $r_{\mathrm{NL}}=6.6 h^{-1} \mathrm{Mpc}$ and $A_{\mathrm{mc}}=1.55$. Here the $z$-bins refers to the four photo- $z$ bins used in the analysis.

\section{Cosmological analysis from ACF full shape}

We performed a Bayesian parameter inference analysis (see chapter 3) using two different approaches for the sampling process from the posterior PDF of the model parameters, the usual Metropolis-Hastings scheme and the parallel version of the stretch move. The model parameters considered on our analysis are the total content of matter $\Omega_{\mathrm{m}}$, the fraction of baryons $f_{\mathrm{b}}:=\Omega_{\mathrm{b}} / \Omega_{\mathrm{m}}$ and the normalization of perturbations $\sigma_{8}$. The rest of the relevant cosmological parameters are held fixed at the WMAP7 values given above. As nuisance parameters we consider the LGs bias on each of the photo- $z$ bins, $b_{1}, b_{2}$, $b_{3}$ and $b_{4}$. However, given the well known degeneracy between $\sigma_{8}$ and the galaxy bias discussed on section 4.1.2 (see figure 4.3), in the present work we constrain its product for each photo- $z$ bin, considering them as the actual nuisance parameters. Then, we can write according to the notation of chapter 3

$$
\boldsymbol{\theta}=\left(\Omega_{\mathrm{m}}, f_{\mathrm{b}}\right)
$$

and

$$
\boldsymbol{\psi}=\left(\sigma_{8} b_{1}, \sigma_{8} b_{2}, \sigma_{8} b_{3}, \sigma_{8} b_{4}\right) .
$$

Note that here $\sigma_{8}$ is assumed to have the same value in all the photo- $z$ bins considered. 
The likelihood function used is given by

$$
\mathcal{L}(\boldsymbol{\Theta})=\mathcal{L}(\boldsymbol{\theta}, \boldsymbol{\psi})=|\mathbf{C}|^{-1 / 2} \exp \left[-\frac{1}{2}\left(\mathbf{d}^{\mathrm{T}} \cdot \mathbf{C}^{-1} \cdot \mathbf{d}\right)\right]
$$

where $\mathbf{d}=\widehat{w}-w(\mathbf{\Theta})$ is a vector constructed with the difference between measured (estimated) ACF, $\widehat{w}$, and its theoretical model, $w(\boldsymbol{\Theta})$, model parameter-dependent. Since the ACF was measured in four photo- $z$ bins, this measured and modeled ACFs are in fact a vector with the information of the four bins, i.e. $\widehat{w}=\left(\widehat{w}_{1}, \widehat{w}_{2}, \widehat{w}_{3}, \widehat{w}_{4}\right)$ and the same for the modeled, and $\mathbf{C}$ is the full theoretical covariance matrix that takes into account correlation between photo- $z$ bins discussed above and given in figure 4.7.

As said above, the sampling process from the posterior PDF for the parameters was done using two different approaches: (a) the implementation of the Metropolis-Hastings algorithm given by the widely used by the cosmology community COSMOMC ${ }^{8}$ code [93] running at the LIneA DES Scientific portal and (b) the implementation of the parallel stretch move on the emcee ${ }^{9}$ [57] code running in the IAG/USP Alphacrucis Cluster with 144 cores.

Our results are displayed in figures 4.8 and 4.9 where the marginalized posterior PDFs for each parameter considered are displayed for the Metropolis-hastings- and parallelstretch move-based samplings respectively. These figures also shows the contours of $68 \%$ and $95 \%$ confidence from the marginalized posterior PDFs for every pair of parameters. In the top right of each figure tables displaying the results found for each parameter are presented. On these tables the second columns label the best fit of each parameter after taking the maximum of the overall posterior PDF. After marginalizing over all the other parameters (see chapter 3 for a discussion on marginalization) the third column displays best fit of the marginalized posterior PDF for each parameter, finally, the fourth column describes the $68 \%$ limit of the marginalized posterior PDF mean for each parameter. All of these results are considered for each of the sampling techniques used. Note that we quote results for all parameters, the ones associated with the cosmological model and the nuisance ones.

\footnotetext{
${ }^{8}$ http://cosmologist.info/cosmomc/

${ }^{9}$ https://github.com/dfm/emcee
} 


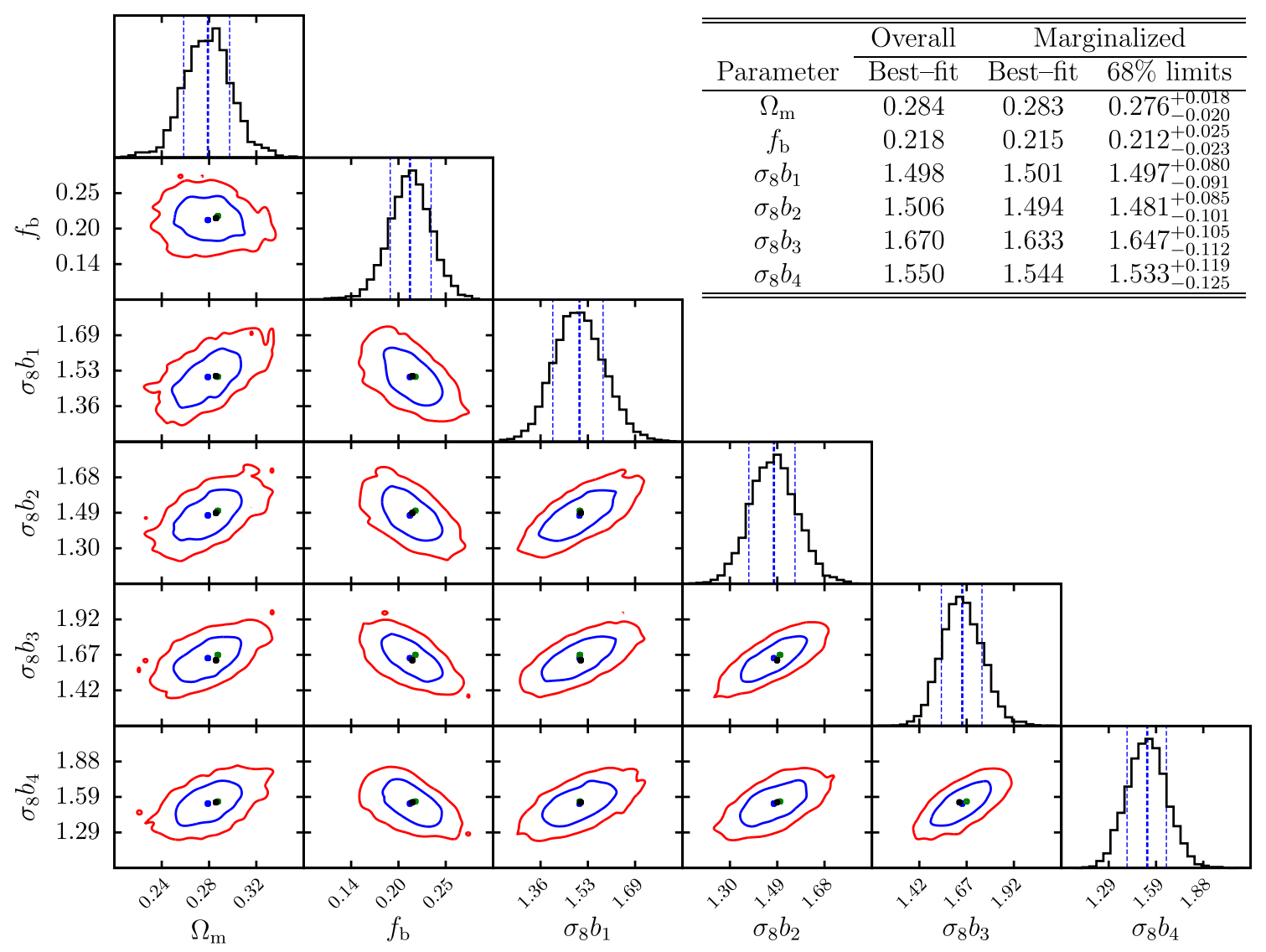

Figure 4.8: Posterior PDFs resulting from the analysis of the ACF of SDSS-III DR8 LGs catalog using the COSMOMC code, i.e. a Metropolis-Hastings algorithm-based sampling technique. The off-diagonal contour plots show the $68 \%(1 \sigma)$, blue lines, and $95 \%$ $(2 \sigma)$, red lines, confidence regions for the two-parameters marginalized posterior PDFs. The circles at the center of each one of these plots show the marginalized mean (blue), marginalized best-fit (black) and overall best-fit (green) values for each parameter. The diagonal plots show the marginalized one-parameter posterior PDFs, the dashed blue lines show the marginalized mean values and $68 \%$ confidence regions. The numerical values of these results are quoted on table on the top right of the figure. 


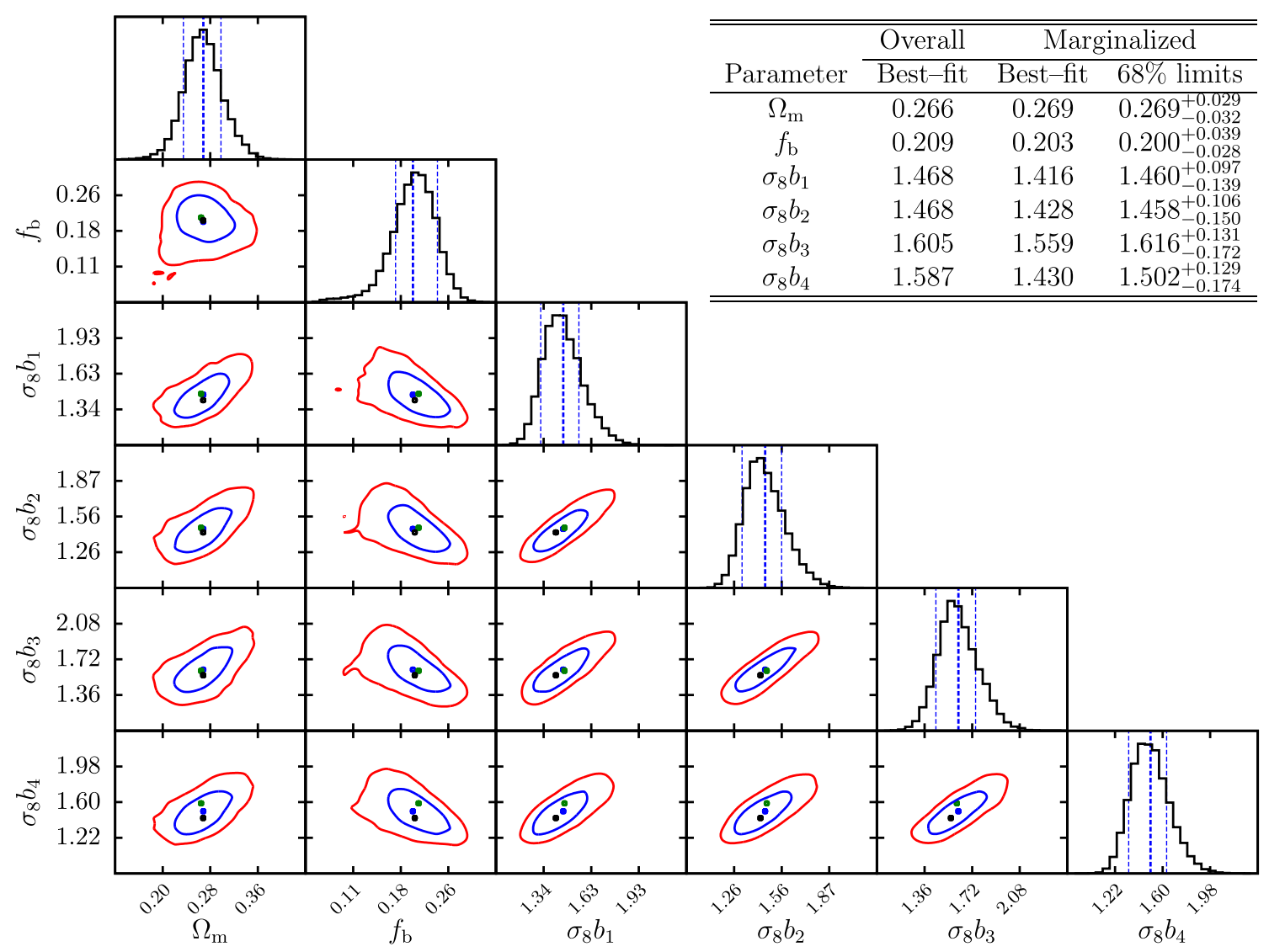

Figure 4.9: Posterior PDFs resulting from the analysis of the ACF of SDSS-III DR8 LGs catalog using the emcee code, i.e. a parallel stretch-move algorithm-based sampling technique. The off-diagonal contour plots show the $68 \%(1 \sigma)$, blue lines, and $95 \%(2 \sigma)$, red lines, confidence regions for the two-parameter marginalized posterior PDFs. The circles at the center of each one of these plots show the marginalized mean (blue), marginalized best-fit (black) and overall best-fit (green) values for each parameter. The diagonal plots show the marginalized one-parameter posterior PDFs, the dashed blue lines show the marginalized mean values and $68 \%$ confidence regions. The numerical values of these results are quoted on table on the top right of the figure.

The marginalized posterior PDF found for all the parameters considered seem to be well behaved in the sense that appear to be consistent with a Gaussian PDF. The results obtained with the two different sampling techniques are consistent with each other within $1 \sigma$ which indicate that our analysis seem to be robust. However, for all the parameters the COSMOMC sampler (MH algorithm-based) gives error bars slightly lower than the emcee sampler (parallel stretch move-based).

In figure 4.10 we show the theory prediction for the ACF in the different photo- $z$ bins evaluated at the mean value for the of the posterior PDF for each parameter, these plots are overlaid on the measured data points. From this figure we can conclude that our modeling for the ACF discussed on section 4.1.2 seems to reproduce fairly well the observed data in the SDSS-III DR8 LGs catalog. 


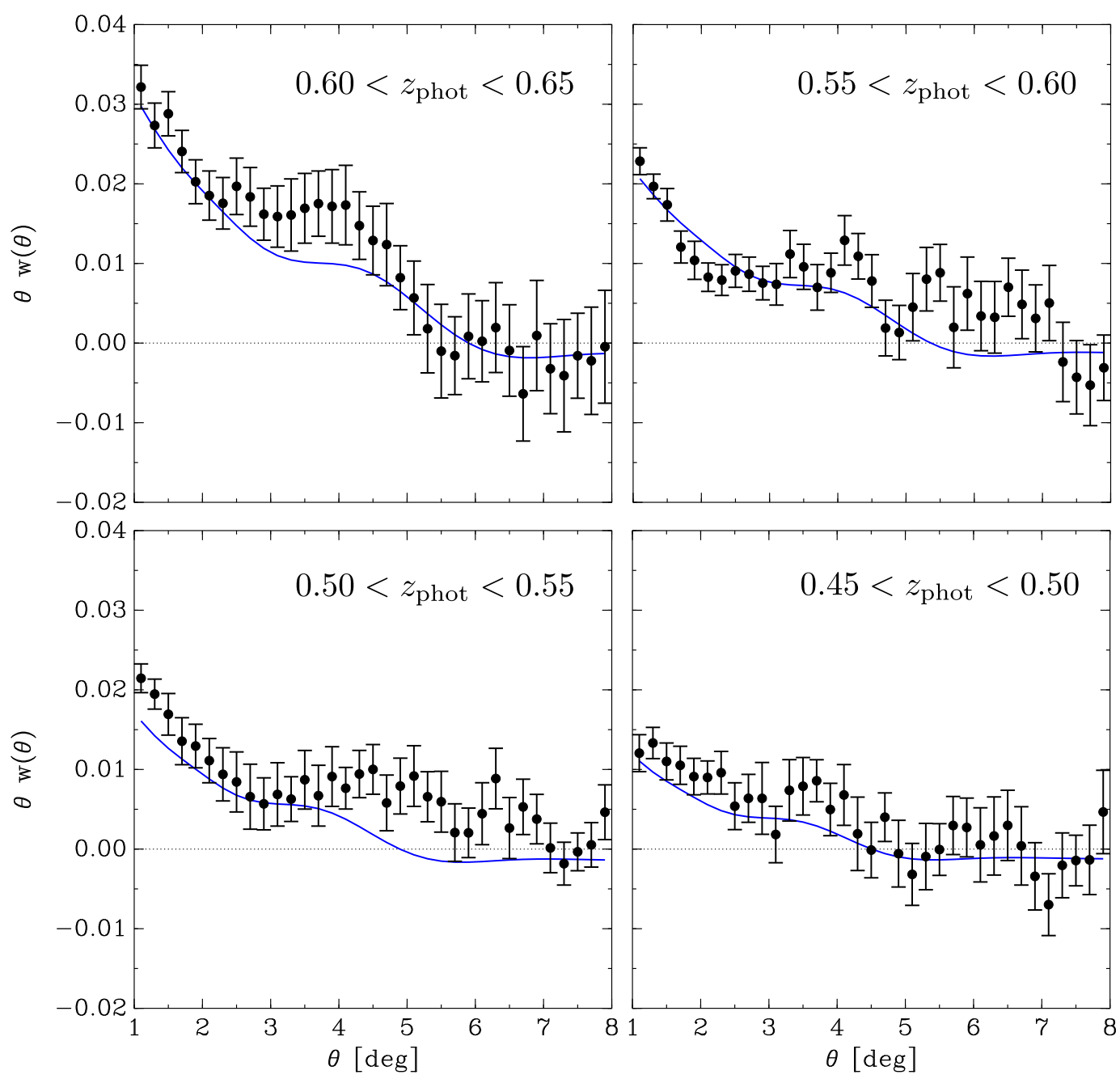

Figure 4.10: Angular correlation functions measured for the SDSS-III DR8 catalog LGs. The error bars correspond to the diagonal elements of the covariance matrix used on the analysis (figure 4.7). The blue lines correspond to the best fit cosmology obtained after a Bayesian inference analysis was performed.

In figure 4.11 we present a comparison for the smoothed PDFs of the cosmological model parameters considered in our analysis $\boldsymbol{\theta}=\left(\Omega_{\mathrm{m}}, f_{\mathrm{b}}\right)$ for the two sampling technique considered overlaid with the actual WMAP7 parameter PDFs (assumed on the computation of the theoretical covariance matrix) which are publicly avaliable by the WMAP team $^{10}$. The figure shows the $68 \%$ coinfidence regions from the PDF of each parameter for each sampling technique and for the WMAP7 result. As stated before, the results of the both sample techniques are consistent with each other and the error bars resulting from COSMOMC method are always slightly lower than the ones coming from emcee. These results are also consistent with the WMAP7 results for the content of total matter in the Universe within $1 \sigma$, but not for the baryon fraction, however allowing for $2 \sigma$ they become consistent.

\footnotetext{
${ }^{10}$ The data can be downloaded at http://lambda.gsf c.nasa.gov/product/map/dr4/m_products.cfm
} 

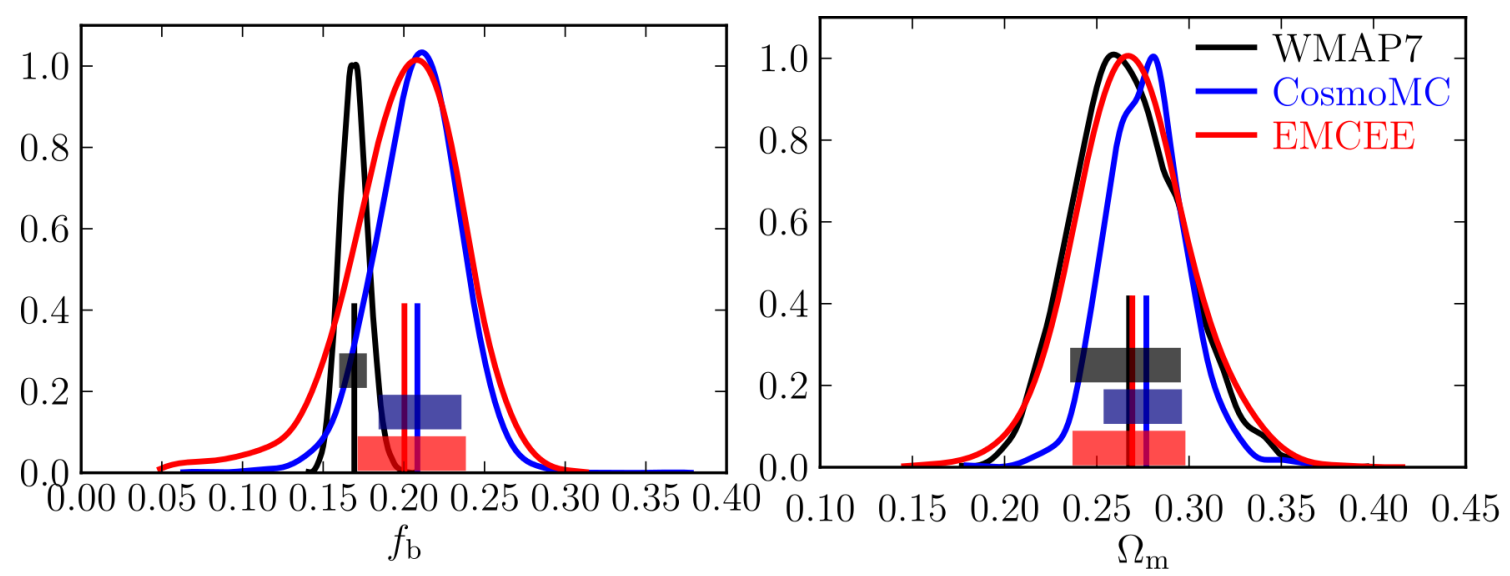

Figure 4.11: Smoothed marginalized posterior PDFs for the cosmological model parameters considered in our analysis of the ACF for the LGs in the SDSS-III DR8 catalog. At the bottom of each plot the $68 \%(1 \sigma)$ coinfidence regions are shown as bars.

Given the fact that the error bars found by COSMOMC sampler are all systematically smaller than those found by emcee sampler for all the parameters considered, while its mean and best-fit values are all consistent with each other, we argue that the error bars from emcee will decrease if we allow to draw more samples in order to have a better statistics in the emcee sampling. By this reason we quote as final constrain results for our analysis the results obtained by using the COSMOMC sampler (figure 4.8).

Although during our analysis we used the WMAP7 parameters defining the fiducial cosmology [72], the Planck collaboration recently released its first cosmological results [17]. The main difference between the model parameters found by this two analysis is on the content of total matter $\Omega_{\mathrm{m}}$ and the Hubble parameter $h$. The former is $\sim 10 \%$ higher with Planck's data and the latter is $\sim 4 \%$ lower. Since $\Omega_{\mathrm{m}}$ is left as a free parameter in our analysis, it is not an issue, but $h$ was fixed to the WMAP7 value. As shown in [81] the major effect of changing $h$ is in the $\Omega_{\mathrm{m}}$ constrain because the clustering characteristics are driven mostly by the combination $\Omega_{\mathrm{m}} h$. Lowering $h$ implies an increase of $\Omega_{\mathrm{m}}$, this degeneracy can also be seen on the figure 4.3. We argue that taking into account this fact our results seem consistent. In fact, since our constrained value for the matter content with WMAP7 Hubble parameter is $\Omega_{\mathrm{m}}=0.28$, if we instead use $h=0.68$, as found by Planck, we would expect to found a higher value, in better agreement with $\Omega_{\mathrm{m}}=0.32$ as quoted by Planck.

It is extremely important to note that the quoted errors of $8 \%$ for $\Omega_{\mathrm{m}}$ and $12 \%$ for $f_{\mathrm{b}}$ are underestimated since they do not take into account the proper marginalization over all the other cosmological parameters that in our analysis were fixed. For more realistic errors, we should have varied all parameters, including the Hubble parameter $h$, spectral index $n_{s}$, the dark energy equation of state parameter $w$ and the spatial curvature $K$, and marginalized over them. Unfortunately the statistical significance of our data set alone is not sufficient to obtain useful constraints. Combining our results with other probes, as for example, the recent CMB Likelihood provided for the Planck collaboration will offer a more complete analysis and therefore better constraints due to the complementarity of these probes. Related to this underestimation of the errors for $f_{\mathrm{b}}$, and the proper effect of taking into account the impact of all cosmological model parameters should be the problem encountered on the higher value encountered for the baryon fraction.

Nonetheless, our results point out that the methods applied to extract information from measurements of ACF in configuration space are able to yield competitive cosmo- 
logical constraints. This indicates that these methods will be even more useful when applied to future data sets with greater constraining power. In fact this will be showed in this work by considering a more complete catalog from simulated data for the DES in the next section. Besides, The combination with other probes of large scale structure and CMB should provide additional consistency checks and even better constraints.

\subsubsection{BCC-Aardvark-v1.0 red galaxies ACF}

Recently two different simulations, part of the so-called Blind Cosmology Challenge (BCC), were provided to the DES collaboration. They are the BCC-Aardvark-v1.0 and the BCC-Buzzard-v1.0 catalogs. The former is a flat $\Lambda$ CDM cosmology simulation with available truth tables for galaxies and halos, whereas the latter is the first catalog of a totally unknown cosmology with only observed quantities provided.

In this section we present some preliminary cosmological results obtained from the analysis of the angular correlation function of red galaxies of the Blind Cosmology Challenge Aardvark-v1.0 catalog.

\section{The catalog}

The BCC-Aardvark-v1.0 catalog was released on April 11, 2013. It is a $10313 \mathrm{deg}^{2}$ (one quarter of the sky) catalog to DES full depth. This represents a semi-blind cosmology challenge since it simulates a flat $\Lambda \mathrm{CDM}$ cosmological model, i.e. it is already known that the dark energy equation of state is described by a parameter $w=1$ and that the constant time hypersurfaces have an Euclidean (flat) geometry, $\Omega_{K}=0$. All the other parameters describing the full FLRW model are unknown. In addition to the so-called observed catalogs, where the true properties of the sources are perturbed by simulated errors, the truth tables for halos and galaxies were also made available. The catalog contains 1.36 billion galaxies passing the DES 5-sigma signal-to-noise limit in at least one DES band. In the catalog release a mask for the $5000 \mathrm{deg}^{2}$ DES footprint was also provided. 


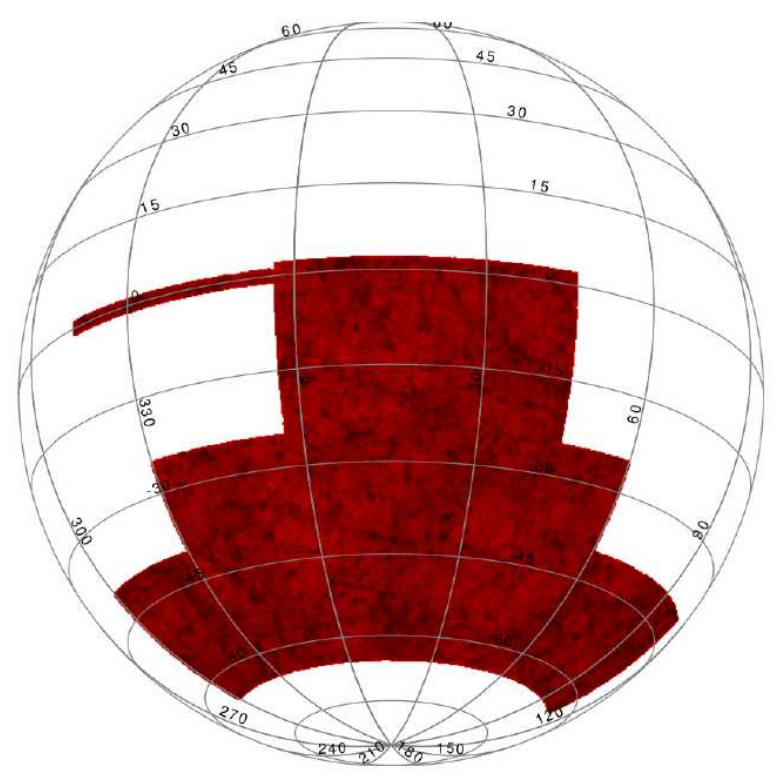

Figure 4.12: Sky coverage in the BCC-Aardvarkv1.0 catalog corresponding to the DES Round82 hybrid footprint, see table 4.3 and text for details.

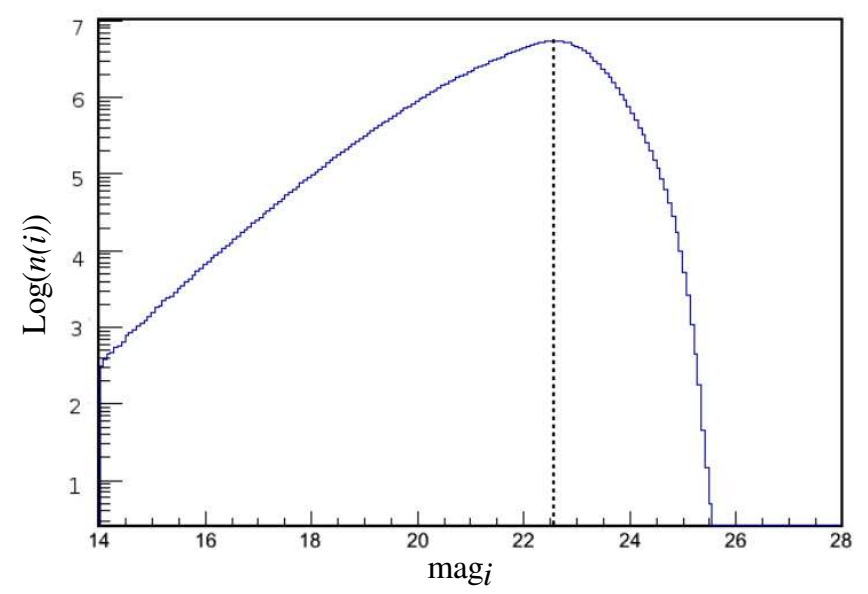

Figure 4.13: Number of objects in the BCC-Aardvarkv1.0 catalog in the $i$-band as a function of the magnitude.

The observed catalogs with DES grizY magnitudes and errors comprise $175 \mathrm{~GB}$ of data and were downloaded from SLAC at approximately 150 Mbps in about 2.5 hours. The $10313 \mathrm{deg}^{2}$ catalog was ingested into LIneA database and then vertically partitioned into seven smaller tables, representing the Round82 DES footprint, to improve the reading performance.

\section{Sample selection}

The VAC (Value-Added Catalog) for large scale angular correlation function analysis was created at the DES science Portal by the DES-Brazil team selecting the DES area according to the Round 82 hybrid footprint. It includes SPT, Viking, Round 82 and Stripe 82 surveys as is shown in table 4.3. The resulting selected BCC footprint is shown in figure 


\begin{tabular}{lcc}
\hline \hline & RA [deg] & DEC [deg] \\
\hline SPT & $-60 \leq \alpha \leq 105$ & $-65 \leq \delta \leq-40$ \\
Viking & $-30 \leq \alpha \leq 60$ & $-40 \leq \delta \leq-25$ \\
Round82 & $-3 \leq \alpha \leq 45$ & $-25 \leq \delta \leq 3$ \\
Stripe82 & $-43 \leq \alpha \leq-3$ & $-1 \leq \delta \leq 1$ \\
\hline \hline
\end{tabular}

Table 4.3: Selection of DES footprint in BCC (Round 82 hybrid). The first column especifies the different surveys composing it, the following two columns gives the corresponding footprint of each one in terms of its limiting angular coordinates on the sky, RA for Right Ascension $(\alpha)$ and DEC for Declination $(\delta)$ (see figure 4.12).

4.12 .

In addition to the limited area described above, it had been applied the mangle mask supplied by the BCC team with bright star holes corresponding to the BCC current stellar catalog.

Besides selecting objects with a signal-to-noise ratio larger than 3 in all bands, we adopted a limiting magnitude of 22.6 in the $i$-band, corresponding to the peak of the counts, in the redshift range $0.2<z_{\text {phot }}<1.4$, see figure 4.15 .

In order to separate Blue (Late) and Red (Early) types of galaxies LePhare photo- $z$ code was used. LePhare was run on a subsample of 100.000 galaxies randomly taken from $\mathrm{BCC}$ in a small area of $100 \mathrm{deg}^{2}$ with coordinates $40<\alpha<50$ and $50<\delta<40$. A suitable set of templates was used, encompassing 21 spectral energy distributions (SED) among those used by [94] in the CFHTLS analysis, covering all galaxy types avaliable (ellipticals, spirals, irregulars and starburst). The best fitting SED was firstly taken to be the galaxy type. Types were grouped as Early (ellipticals) and Late (spirals, irregulars and starbursts), consistently with the expected distribution in a color $(g-r)$ versus $i$ magnitude diagram of real data (CFHTLS). Using a plot showing the color $(g-r)$ versus redshift, a separation line between Early and Late types was visually estimated in redshift bins and a second order polynomial was fitted to the line. The final galaxy classification as Early or Late was made if the galaxy is redder or bluer than the separation line according to figure 4.14 and obey the following equation:

$$
(g-r)_{\text {sep }}=-1.215 z^{2}+1.795 z+0.8607 .
$$

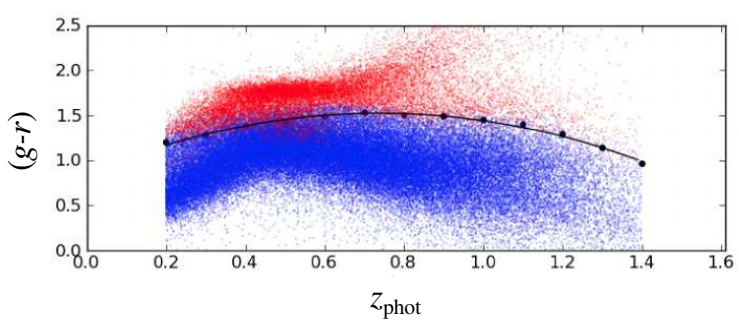

Figure 4.14: BCC-Aardvarkv1.0 galaxy type separation into early-type, ellipticals (red) and late-type, spirals, irregulars, starbursts (blue). The separation was done following the criteria of (4.68).

The In this way, de definition for each type follows

$$
\text { Early: } \quad(g-r)>(g-r)_{\text {sep }}, \quad \text { Late: } \quad(g-r)<(g-r)_{\text {sep }},
$$


In an over-simplified approach, we assumed during this analysis the conditional probability for a measured a photometric redshift $z_{\text {phot }}$ of a given galaxy to corresponds to a true redshift $z, P\left(z \mid z_{\text {phot }}\right)$, which is related to the error in photo- $z$ measurements and to intrinsic magnitude-redshift degeneracies, to be Gaussian according to equation (4.44). In addition zero bias $\left(z_{\text {bias }}=0\right)$ was also assumed, so that all photometric uncertainties were assumed to be given in terms of the photo- $z$ error dispersion (scatter) which was assumed to have an usual redshift dependence given by $\sigma_{z}=\sigma_{0}(1+z)$, where the photo- $z$ error at redshift zero, $\sigma_{0}$, was estimated from the BCC catalog itself, following the method developed by [95]. This empirical method is based on the assumption that close pairs of galaxies on the sky have a significant probability of being physically associated, and therefore, of lying at nearly the same redshift. In such a way that, the difference in photometric redshifts in close pairs is therefore a measure of the redshift uncertainty. Applied to the red and blue galaxy sample, this method yields $\sigma_{0}^{\text {red }}=0.024$ and $\sigma_{0}^{\text {blue }}=0.027$, respectively.

Since the BCC catalog supplies the photometric redshift information for each galaxy, the number of galaxies $N(z)$ per unit of true redshift $z, n(z)=\mathrm{d} N(z) / \mathrm{d} z$, was estimated for the red and blue galaxies samples according to equation (4.41) where $\mathrm{d} N\left(z_{\text {phot }}\right) / \mathrm{d} z_{\text {phot }}$ is the number of galaxies per unit of photometric redshift, obtained by simply binning galaxies in photo- $z$ on the catalog.

Using the estimated number density of galaxies, in figure 4.15 the selection functions for the photo- $z$ bins considered in this analysis are shown. This selections were computed according to equation (4.42), where the window function in photo- $z$ takes into account only the binning process, i.e. it is given by equation (4.43). Hereinafter we restrict our analysis to the red sample catalog obtained with the methods discussed above.

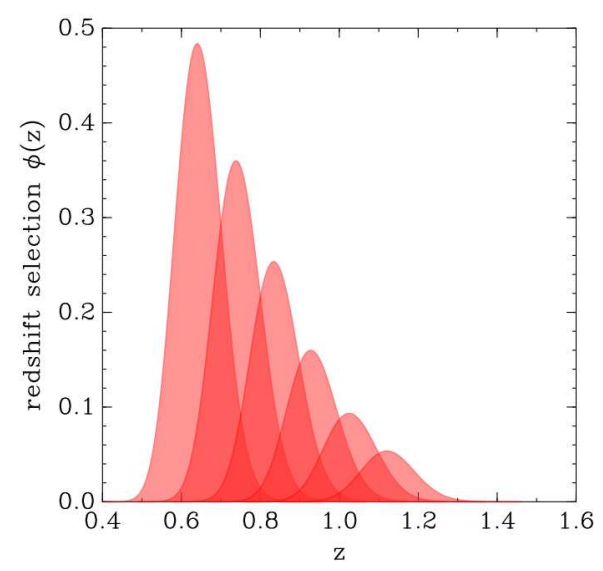

Figure 4.15: Non-normalized selection functions as function of the true redshift $z$ for the red galaxy sample of the BCC-Aardvark-v1.0 for bins of $\Delta z_{\text {phot }}=0.1$ over the range $0.6<z_{\text {phot }}<1.2$. These selection functions account for a Gaussian approximation of photo- $z$ errors.

The photo- $z$ dispersion and the number of galaxies in each of the four photo- $z$ bins used in this work are displayed in table 4.4 


\begin{tabular}{c|cc}
\hline \hline$z$-bin & photo- $z$ range & Number of red galaxies \\
\hline 1 & {$[0.2,0.3]$} & 1.683 .345 \\
2 & {$[0.3,0.4]$} & 3.561 .715 \\
3 & {$[0.4,0.5]$} & 4.674 .176 \\
4 & {$[0.5,0.6]$} & 4.206 .681 \\
5 & {$[0.6,0.7]$} & 3.286 .862 \\
6 & {$[0.7,0.8]$} & 2.551 .088 \\
7 & {$[0.8,0.9]$} & 1.846 .046 \\
8 & {$[0.9,1.0]$} & 1.146 .531 \\
9 & {$[1.0,1.1]$} & 682.652 \\
10 & {$[1.1,1.2]$} & 400.139 \\
11 & {$[1.2,1.3]$} & 191.405 \\
12 & {$[1.3,1.4]$} & 79.534 \\
\hline \hline
\end{tabular}

Table 4.4: Photometric redshift bins used in the analysis of the ACF of BCC-Aardvarkv1.0 red galaxies catalog.

\section{Measuring and modeling the BCC-Aardvark ACF}

We measure the angular correlation function in the selected red galaxy sample using the Landy-Szalay pair counting-based method (see section 2.1.2). In order to speed the computation we use the $\mathrm{CUTE}^{11}$ code [96], a robust parallel implementation of the LS measurement method, running in the LIneA DES Science Portal. We analyzed red galaxies in 6 photo- $z$ bins of width $\Delta z_{\text {phot }}=0.1$ on the range $0.6<z_{\text {phot }}<1.2$. In all the photo- $z$ bins the ACF was measured on 30 angular bins of width $\Delta \theta=0.2$ deg on the range $1 \mathrm{deg}<\theta<7 \mathrm{deg}$, such intervals were chosen in order to approximately guarantee that the analysis is done on large scales, i.e. for comoving distances in the range $20<r<200 h^{-1} \mathrm{Mpc}$ on all photo- $z$ bins.

The model for the ACF used during this analysis is the one described in section 4.1.2, i.e. the distant observer approximation is assumed along with the spatial flatness of the FLRW cosmological model used to describe the observations. The latter assumption is exact for the BCC-Aardvark-v1.0 catalog since was assumed for the simulation. All the relevant effects were taken into account, photo- $z$ uncertainties, according to the discussion in the previous paragraph, nonlinearities in the clustering of galaxies, redshift-space distortions and galaxy biasing. For the nonlinearities it was assumed $r_{\mathrm{NL}}=6.6 h^{-1} \mathrm{Mpc}$ and $A_{\mathrm{mc}}=1.5$ in the RPT parametrization. The galaxy biasing effect (see the begining of $\$ 1.4 .1$ for some discussion) was modeled according to the following parametrization

$$
b_{\mathrm{g}}(z)=b_{0}+b_{1} z+b_{2} z^{2},
$$

i.e. as a second order polynomial in redshift around $z=0$.

In order to perform the cosmological analysis, we modeled theoretically the ACF covariance matrix according to section 4.1.2, i.e. assuming Gaussianity at large scales as given by equation (4.53). This approximation is in very good agreement with the covariance obtained from N-body simulations, see e.g. [8]. Note that our model for the ACF covariance properly account for partial sky coverage and shot noise as well as for the same effects considered for the ACF modeling, namely RSD, biasing, nonlinearities and the photo- $z$ effects. However, we do not allow for a cosmology-dependent covariance analysis

\footnotetext{
${ }^{11}$ http://members.ift.uam-csic.es/dmonge/CUTE.html
} 
because of computational time reasons, so we need to fix the cosmological parameters to a fiducial model and choose an appropriate value for the galaxy bias according to the galaxy sample. As a fiducial cosmology we adopt a flat CDM universe with the parameters: $\Omega_{\mathrm{m}}=0.272, \Omega_{\mathrm{b}}=0.0456, n_{s}=0.963, \sigma_{8}=0.801, h=0.704$ and $w_{\mathrm{DE}}=-1$. We also fix nonlinear RPT parametrization parameters to $r_{\mathrm{NL}}=6.6 h^{-1} \mathrm{Mpc}$ and $A_{\mathrm{mc}}=1.55$ as for the ACF. The galaxy bias is also parametrized according to equation (4.69). In order to determine the appropriate values for the coefficients $b_{0}, b_{1}$ and $b_{2}$ on (4.69) we adopted the following strategy: First we fixed the parameters to the fiducial cosmology and find the best fit for the growth factor and galaxy bias (redshift space distortions methodology) considering $\sigma_{8}=0.801$ for all the photo- $z$ bins. Here we are not interested on discuss in detail this method, we refer the interested reader to [12]. For the red galaxies catalog the obtained coefficients are $b_{0}=1.90, b_{1}=0$ and $b_{2}=-1.67$. With the ingredients listed above the resulting theoretical covariance matrix used on our analysis is shown on figure 4.16 .

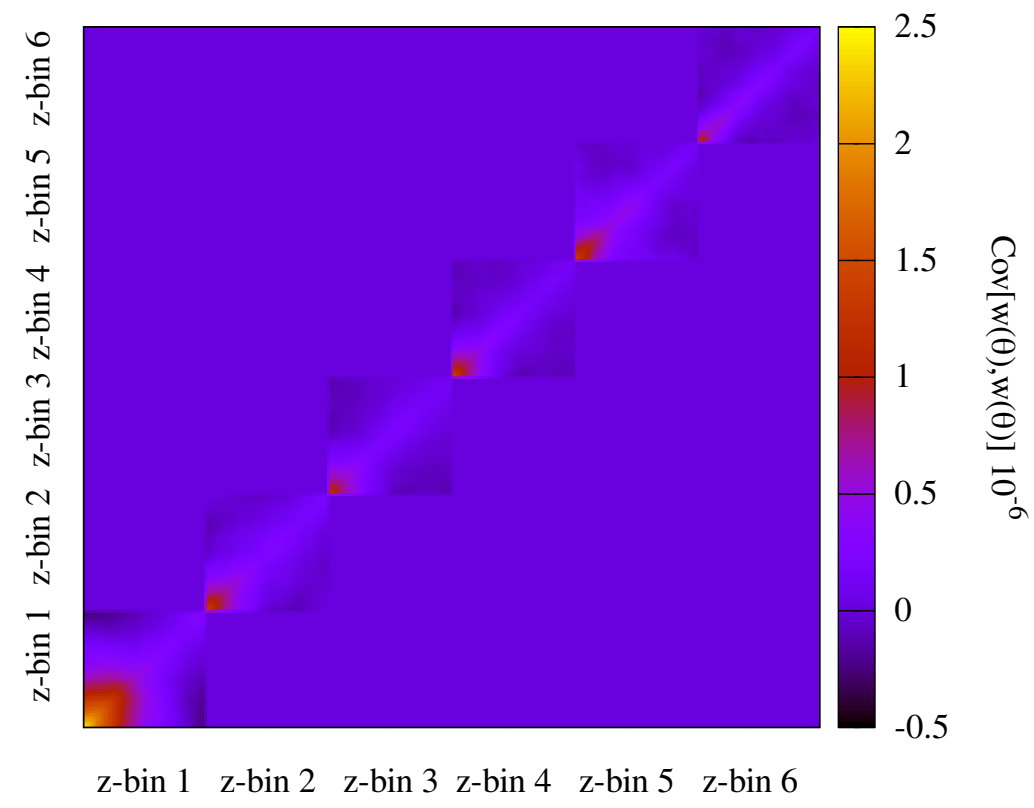

Figure 4.16: Theoretical ACF covariance matrix used on the BCC analysis. Since we do not allow for an analysis with a cosmology-dependent theoretical covariance because of computational time reasons, we fix the theoretical covariance to a flat FLRW model with parameters $\Omega_{\mathrm{m}}=0.272, \Omega_{\mathrm{b}}=0.0456, n_{s}=0.963, \sigma_{8}=0.801, h=0.704$ and $w_{\mathrm{DE}}=-1$. The galaxy bias is assumed to be given by equation (4.69) with coefficients $b_{0}=1.90, b_{1}=0$ and $b_{2}=-1.67$. Also nonlinearities are fixed according to $r_{\mathrm{NL}}=6.6 h^{-1} \mathrm{Mpc}$ and $A_{\mathrm{mc}}=1.55$. Here the $z$-bins refers to the six photo- $z$ bins used in the analysis.

Our corresponding results for the measurements of the ACF en each photo- $z$ bin are shown in black in figure 4.18, where the error bars correspond to the diagonal elements on the covariance matrix (figure 4.16).

\section{Cosmological analysis from ACF full shape}

We performed a Bayesian parameter inference analysis (see chapter 3) using the parallel version of the stretch move for sampling the posterior PDF of the model parameters. The model parameters considered on our analysis are the total content of matter, the content 
of baryons, the primordial power spectrum spectral index and amplitude of perturbations, and the Hubble factor, so that (see chapter 3 for the notation used)

$$
\boldsymbol{\theta}=\left(\Omega_{\mathrm{m}}, \Omega_{\mathrm{b}}, n_{s}, A_{s}, h\right) .
$$

As nuisance parameters we consider the introduced coefficients of the parametrization for the galaxy bias, equation (4.69).

$$
\boldsymbol{\psi}=\left(b_{0}, b_{1}, b_{2}\right)
$$

during our analysis we also quote the results of an important derived parameter of the model, the variance of linear fluctuations at $8 h^{-1} \mathrm{Mpc}, \sigma_{8}$.

The likelihood function used is given by

$$
\mathcal{L}(\boldsymbol{\Theta})=\mathcal{L}(\boldsymbol{\theta}, \boldsymbol{\psi})=|\mathbf{C}|^{-1 / 2} \exp \left[-\frac{1}{2}\left(\mathbf{d}^{\mathrm{T}} \cdot \mathbf{C}^{-1} \cdot \mathbf{d}\right)\right],
$$

where $\mathbf{d}=\widehat{w}-w(\boldsymbol{\Theta})$ is a vector constructed with the difference between measured (estimated) ACF, $\widehat{w}$, and its theoretical model, $w(\boldsymbol{\Theta})$, model parameter-dependent. Since we measured the ACF in six photo- $z$ bins, this measured and modeled ACFs are in fact a vector with the information of the six bins, i.e. $\widehat{w}=\left(\widehat{w}_{1}, \widehat{w}_{2}, \widehat{w}_{3}, \widehat{w}_{4}, \widehat{w}_{5}, \widehat{w}_{6}\right)$ and the same for the modeled, and $\mathbf{C}$ is the full theoretical covariance matrix that takes into account correlation between photo- $z$ bins (figure 4.16).

The sampling process from the posterior PDF for the parameters was done using the implementation of the parallel stretch move on the emcee ${ }^{12}$ [57] code running in the IAG/USP Alphacrucis Cluster with 144 cores.

Ours results are displayed in table 4.5. The second column labels the best fit of each parameter after taking the maximum of the overall posterior PDF. After marginalizing over all the other parameters (see chapter 3 for a discussion on marginalization) the third column displays best fit of the marginalized posterior PDF for each parameter, finally, the fourth column describes the $68 \%$ limit of the marginalized posterior PDF mean for each parameter. Note that we quote results for all parameters, the ones associated with the cosmological model, the nuisance and the derived parameters.

\begin{tabular}{cccc}
\hline \hline & Overall & \multicolumn{2}{c}{ Marginalized } \\
\cline { 2 - 4 } Parameter & Best-fit & Best-fit & $68 \%$ limits \\
\hline$\Omega_{\mathrm{m}}$ & 0.254 & 0.240 & $0.238_{-0.014}^{+0.014}$ \\
$\Omega_{\mathrm{b}}$ & 0.056 & 0.045 & $0.047_{-0.006}^{+0.005}$ \\
$n_{s}$ & 0.905 & 0.996 & $0.998_{-0.065}^{+0.052}$ \\
$A_{s}\left(10^{-9}\right)$ & 1.570 & 2.122 & $2.090_{-0.320}^{+0.272}$ \\
$h$ & 0.885 & 0.704 & $0.730_{-0.072}^{+0.051}$ \\
$b_{0}$ & 3.064 & 2.437 & $2.491_{-0.432}^{+0.481}$ \\
$b_{1}$ & -3.664 & -2.222 & $-2.232_{-1.322}^{+0.957}$ \\
$b_{2}$ & 3.403 & 2.516 & $2.627_{-0.391}^{+0.780}$ \\
$\sigma_{8}$ & 0.748 & 0.734 & $0.740_{-0.070}^{+0.058}$ \\
\hline \hline
\end{tabular}

Table 4.5: Results for the ACF analysis of the BCC-Aardvarkv1.0 red galaxy sample. The second column labels the best fit of each parameter after taking the maximum of the overall posterior PDF, the third column displays best fit of the marginalized posterior PDF for each parameter and the fourth column describes the $68 \%$ limit of the marginalized posterior PDF mean for each parameter.

${ }^{12}$ https://github.com/dfm/emcee 
The figure 4.17 shows the marginalized posterior PDFs for each parameter considered, including nuisance and derived ones. This figure also shows the contours of $68 \%$ and $95 \%$ confidence from the marginalized posterior PDFs for every pair of parameters. Except for the $b_{1}$ parameter, the marginalized posterior PDFs found for all the parameters are well behaved, in the sense that appears to have a Gaussian form. Thus the actual cosmological model parameters $\theta$ were all found to be well behaved.

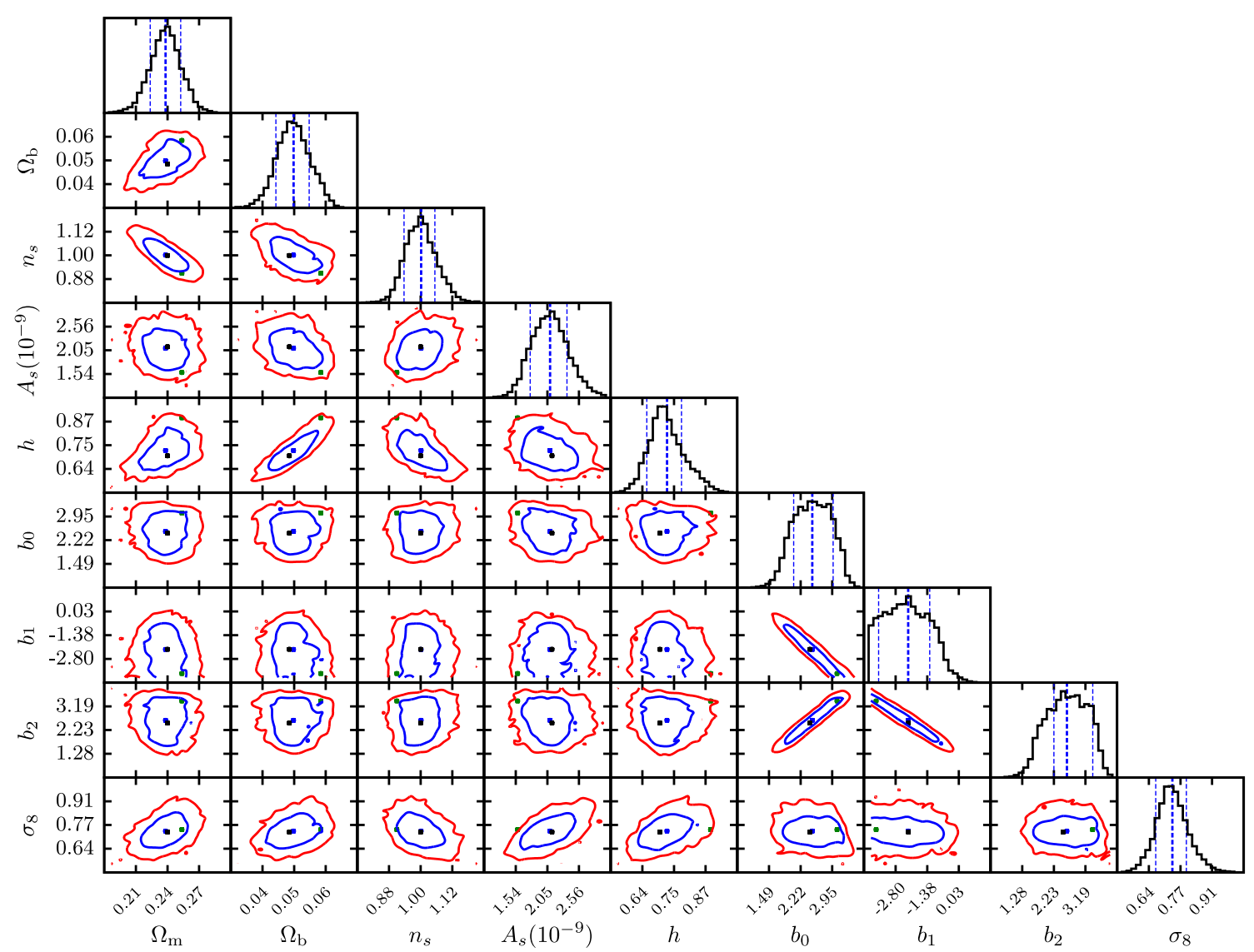

Figure 4.17: Posterior PDFs resulting from the analysis of the ACF of BCC-Aardvarkv1.0 red galaxies. The off-diagonal contour plots show the $68 \%(1 \sigma)$, blue lines, and $95 \%$ $(2 \sigma)$, red lines, confidence regions for the two-parameters marginalized posterior PDFs. The circles at the center of each one of these plots show the marginalized mean (blue), marginalized best-fit (black) and overall best-fit (green) values for each parameter. The diagonal plots show the marginalized one-parameter posterior PDFs, the dashed blue lines show the marginalized mean values and $68 \%$ confidence regions. The numerical values of these results are quoted on table 4.5.

In figure 4.18 we show the theory prediction for the ACF in the different photo- $z$ bins evaluated at the mean value of the posterior PDF for each parameter, these plots are overlaid on the measured data points. From this figure we can conclude that our modeling for the ACF discussed on section 4.1.2 seems to reproduce fairly well the observed data. 

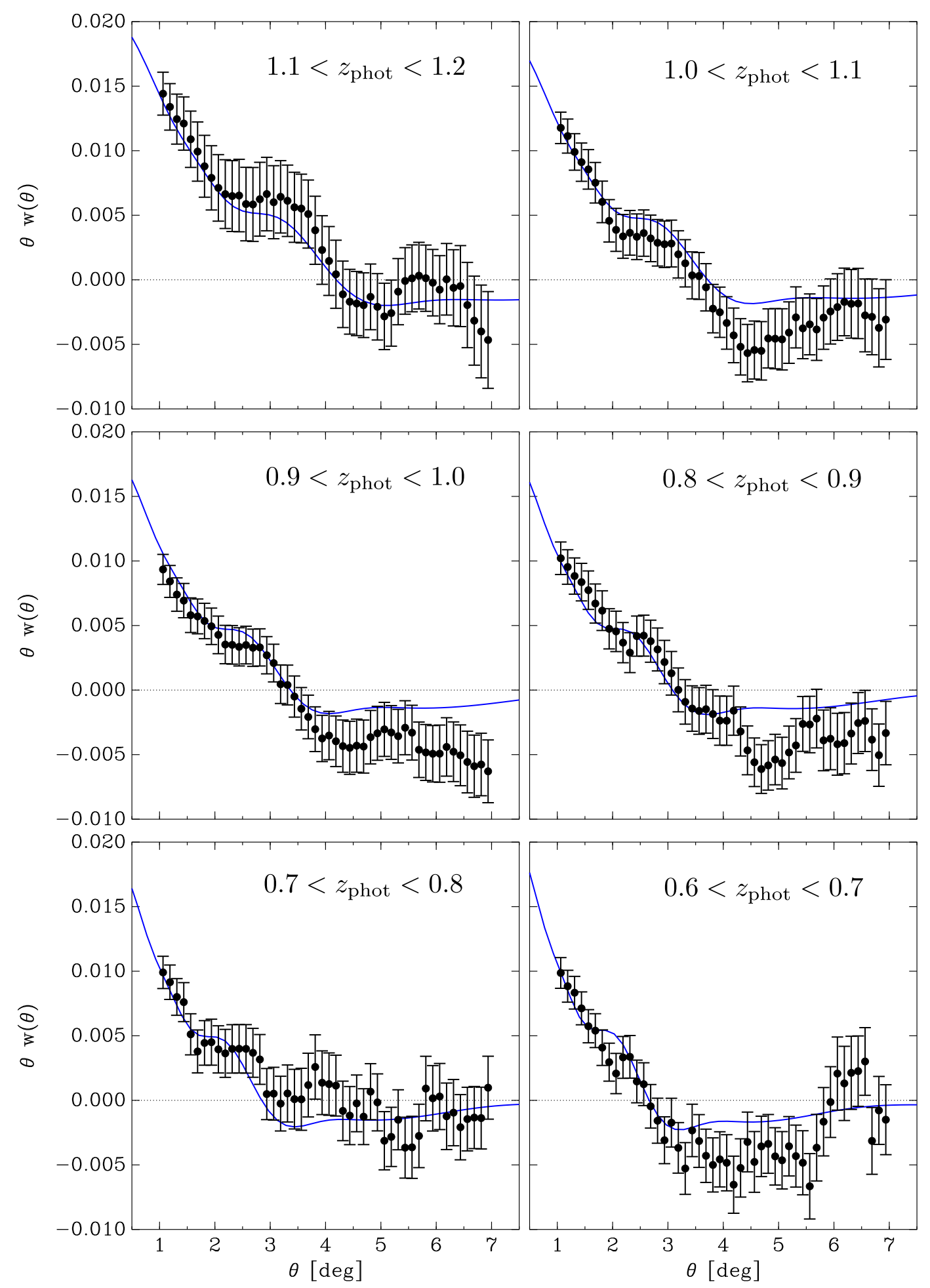

Figure 4.18: Angular correlation functions measured for the red galaxies in the BCCAardvark-v1.0 catalog. The error bars correspond to the diagonal elements of the covariance matrix used on the analysis (figure 4.16). The blue lines correspond to the best fit cosmology obtained after a Bayesian inference analysis was performed.

We stress that our results are quite preliminary at this point. However, the true cosmological values for Aardvark-v1.0 (the ones used on the simulations) where made available to the DES-Brazil team recently, and we are certain that our results are consistent with all of them within $1 \sigma$ confidence, except for $\sigma_{8}$. We argue that this could be related to the 
obtained behavior of the posterior PDF for $b_{1}$ (one of the coefficients used to parametrize the galaxy bias) because of the well known degeneracy between $\sigma_{8}$ and the galaxy bias discussed on section 4.1.2 (see figure 4.3). We hope to repeat these analysis to obtain a better understanding of this issues. One possible variation is to use other parametrization or a better physically-motivated model for the bias. Along, of course, with understanding of potential problems with our methods and codes. In any case, the fact that just one parameter results inconsistent with the actual simulation parameters indicates that our analysis is robust.

From this preliminary analysis many steps remain for the future, one initial further step is the inclusion of more realistic photo- $z$ uncertainties, one fundamental issue for the application of our methods to the real data coming. This will be done by replacing the assumption of Gaussian photo- $z$ errors by real analysis of photo- $z$ s based on current available codes using techniques as machine learning or template fitting formulas.

Another further step is related to the proper inclusion of the spatial curvature as a model parameter in the analysis. We can see, for example, if after its inclusion we still obtain results consistent with the ones presented here and with the fact that Aardvarkv1.0 catalog is based on a simulation of a spatially flat FLRW model. To this point the present work offers the basic tools, the remaining work is related with the proper implementation of the theoretical developments presented here.

Once these issues have been understood and worked out, we will repeat these studies for the BCC-Buzzard-v1.0, a truly blind catalog, where even the spatial curvature is unknown.

One further step that remains as an interesting challenge is the consistent combination of the different probes. This dissertation also present some preliminary work related with weak lensing-based probes that we want to properly implement and combine with the analysis of the $\mathrm{ACF}$ in the future. But we are also interested on combination with other DES probes as, for example, the abundance of galaxy clusters which is currently receiving much attention inside the DES-Brazil group. We want to properly including the correlations between probes in order to better constrain cosmological parameters.

\subsection{Two-point statistics in harmonic space}

In $\S 2.2$ the two-point angular power spectrum $C_{\ell}$ for arbitrary $3 \mathrm{D}$ fields projected in the observer sky in FLRW Universe models was found in terms of the underlying power spectrum of the 3D field, equations (2.15) and (2.21).

In the present section some preliminary work on the application of this theoretical results to the modeling of the three two-point statistics that can be considered from observations of weak lensing and clustering of galaxies on large scales, namely the autoand cross-correlations of lensing and galaxy positions, is presented. In particular we use the naive estimator for the angular power spectra developed in section 2.2.2 to measure this three APSs on simulated data from the Onion simulations [13]. This simulations owe their names to the fact that are design to offer catalogs picturing the universe as a set of concentric radial shells of finite width around the observer, i.e, an onion-like structure. Such catalogs are very useful in the context of modern wide-area photometric galaxy surveys, like the DES, where a fundamental characteristic is the lack of radial accuracy and then splitting the data into redshift shells will be a standard method in the analysis. 


\subsubsection{The onion simulation maps}

The Onion simulations [13] are the first of a new generation of very large scale N-body simulations developed at the Marenostrum supercomputer in Barcelona using the GADGET2 code. Such simulations are named as Marenostrum Institut de Ciencies de l'Espai or MICE simulations and have been being developed for studying clustering statistics of the large scale structure in the context of the modern wide-area photometric galaxy redshift surveys.

In this work we use the first reported of such simulations. A simulation with $2048^{3}$ dark-matter particles in a box-size of $L_{b o x}=3072 \mathrm{Mpc}$, that assume a flat concordance LCDM model with $\Omega_{\mathrm{m}}=0.25, \Omega_{\Lambda}=0.75, \Omega_{\mathrm{b}}=0.044, n_{s}=0.95, \sigma_{8}=0.8$ and $h=0.7$. The resulting particle mass is $M=2.34 \times 10^{11} M_{\odot}$ using a softening length of $50 \mathrm{Kpc} / \mathrm{h}$. Thus, the Onion simulation has a dynamic range close to five orders of magnitude: from Gpcs to tens of Kpcs. The run start at $z_{i}=50$ displacing particles using the Zeldovich dynamics. Noticible is that this MICE simulation has a similar number of particles to the Millenium simulation but $6^{3}=216$ times more volume (and corresponding larger particle mass). This makes the MICE simulation more adequate than the Millenium for very large scale statistical analyses, such as the search of the baryon acoustic scale [13].

In order to mimic the onion-like structure of real data from galaxy surveys the these first MICE simulations were used to built an all-sky light-cone which in turn had been compressed into a set of radial shell maps of given redshift resolution. These all-sky angular maps have been pixelized using the convenient HEALPIX tessellation [52] with high spatial resolution, $N_{\text {side }}=2048$, which pixelices the sky with $12 N_{\text {side }}^{2} \approx 50$ million cells of size $\theta_{\text {pix }} \simeq 1.7$ arcmin size ${ }^{13}$. Noticeable from this novel approach is that it provides an effectively lossless method to compress simulated data by a factor $\sim 1000$ for arcminute resolution angular maps [13]. This allows Terabyte-sized simulations containing tens of billions of particles to be analyzed in a regular laptop.

\subsubsection{Angular power spectra measurements and modeling}

We will adopt the HEALPIX conventions [52]. We are also assuming full sky coverage. This assumption will be adopted throughout this work because it is valid in the case of the simulated Onion maps considered. We also implicitly assumed that the power spectrum of the pixelized map $\mathbf{x}$ was equal to that of the continuous signal $x$. This approximation is only true at high resolution when the pixel size is small compared with $180 / \ell$, and the integrals in the spherical harmonic transform are correctly approximated by matrix multiplications through quadrature [52]. Here we will be using the same resolution of the Onion maps, $N_{\text {side }}=2048$, which pixelices the sky with $12 N_{\text {side }}^{2} \approx 50$ million cells of size $\theta_{\text {pix }} \simeq 1.7$ arcmin size.

In this work we consider two pixelized signals from Onion simulations, the total matter density fluctuations at mean redshift 0.45 and width 0.1 , i.e. dark matter particles in the simulation with redshift between 0.4 and 0.5 and the weak lensing convergence by large scale structure in the Universe of sources at $z_{s}=0.993$. This two signals are associated with the fields studied on section 1.4. For the case of angular clustering, since we are dealing directly with DM particles in the simulation we can assume no biasing effect, i.e. $b_{\mathrm{g}}=1$.

\footnotetext{
${ }^{13}$ Projected matter density and weak lensing maps from the MICE simulations are publicly available at http://www.ice.cat/mice
} 
The two point angular auto- and cross-correlation power spectra of this two signals were estimated using the method depicted on 2.2.2, i.e. by using the ANAFAST routine of HEALPix for 20 values of $\ell$ between $\ell=5$ and $\ell=250$. Such scales are considered in order to guarantee that we are working on large scales were the Gaussian approximation is expected to hold and that the effect of shot noise can be considered negligible, see [13] for a discussion about this point.

Thus, the measurements of the PASs in our analysis are assumed to be uncorrelated and having a Gaussian distribution, and so a variance given by equation (2.68). This over-simplification of the problem constitutes a first approximation to test our methods. As stated before, the present work does not intend to be compete by no means, by the contrary it constitutes a very preliminary first approximation to the real application of the methods developed on section 2.2. The results for the power spectra considered are shown on figure 4.21 with dark filled circles, the error shown in the figures as stated above corresponds to the theoretical Gaussian error.

The model assumed for the APSs in this analysis is the one described in section (2.2), i.e. basicaly a spatially flat FLRW model. The effect of redshift space distortions is not taken into account.

\subsubsection{Simple cosmological analysis of APSs from Onion simula- tions}

We begin by showing the essential ingredients on the theoretical modeling of the weak lensing convergence and angular clustering of matter, namely, its corresponding window functions (1.63) and (1.87) for the Onion Universe Cosmology parameters. Our results are shown on figure 4.19
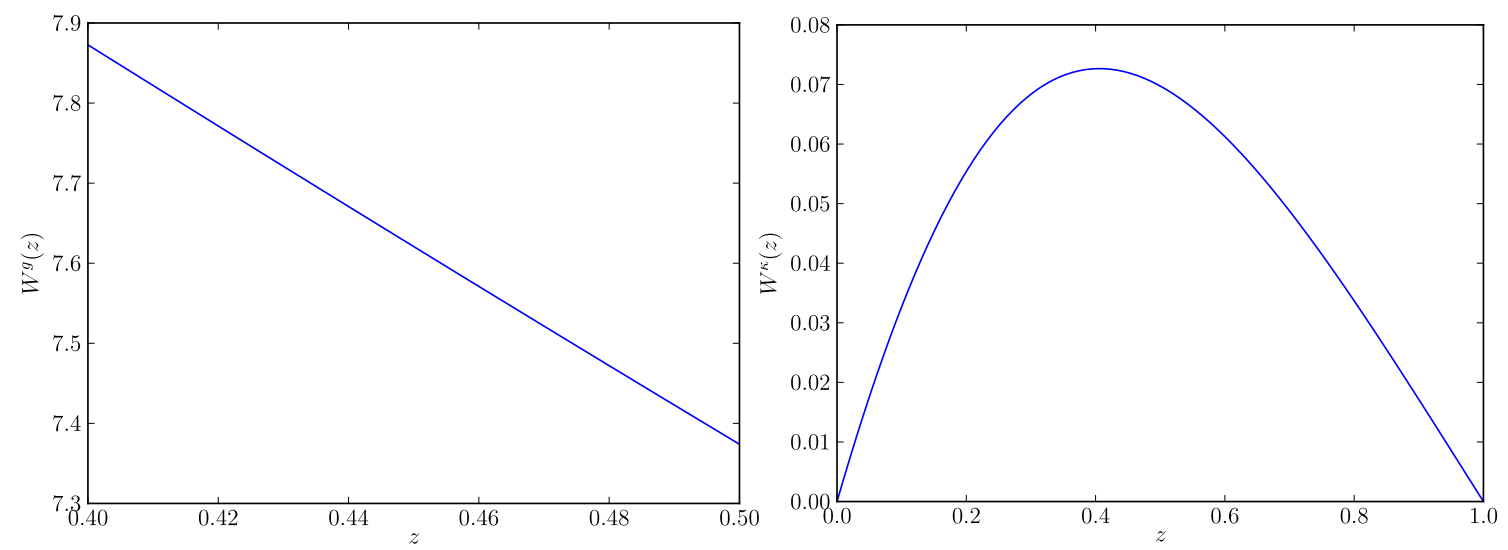

Figure 4.19: Left panel shows the window function for the projected total matter fluctuation in the Onion simulations on a redshift shell with mean redshift 0.45 and width 0.1 (equation (1.63) with $b_{\mathrm{g}}=0$ ), assumed to have constant density in the redshift shell. Right panel shows the weak lensing convergence window function for sources at $z_{s}=0.993$ (equation (1.87)) in the Onion simulation

In the matter case, since the distribution in the shell is considered is homogeneous, the behavior of the window is dominated by the growth function term (see equation (1.63)). In the convergence case, the behavior is the expected for sources located at an specific redshift (see e.g. section 1.4.2 and/or [11]). 
We perform a Bayesian parameter inference analysis using the emcee sampler. The model parameters considered in our analysis are the total content of matter $\Omega_{\mathrm{m}}$, the fraction of baryons $\Omega_{\mathrm{b}}$ and the normalization of perturbations $\sigma_{8}$, i.e.

$$
\boldsymbol{\theta}=\left(\Omega_{\mathrm{m}}, f_{\mathrm{b}}, \sigma_{8}\right)
$$

Note that no nuisance parameters are needed. The remaining parameters for the model are fixed to the actual Onion cosmology.

The likelihood function used in the analysis was assumed Gaussian,

$$
\mathcal{L}(\boldsymbol{\Theta})=\mathcal{L}(\boldsymbol{\theta}, \boldsymbol{\psi})=|\mathbf{C}|^{-1 / 2} \exp \left[-\frac{1}{2}\left(\mathbf{d}^{\mathrm{T}} \cdot \mathbf{C}^{-1} \cdot \mathbf{d}\right)\right]
$$

where $\mathbf{d}:=\widehat{C}_{\ell}^{\mathrm{gg}}-C_{\ell}^{\mathrm{gg}}(\boldsymbol{\theta})$ represents the vector with the difference between the estimated and modeled galaxy position APS (in fact matter fluctuations instead of galaxies in the context of the Onion simulations) and $\mathbf{C}$ is the covariance matrix associated, assumed Gaussian and then diagonal containing the variance of equation (2.68).

Note that this is not the better approach to the problem, a better possibility could be, for example, split the sky in different equal area disjoint regions and measure the $C_{\ell}$ using some Jacknife method. However, the exercise in this section was done only to test our methods.

Our results are presented in table 4.6. The second column labels the best fit of each parameter after taking the maximum of the overall posterior PDF. After marginalizing over all the other parameters (see chapter 3 for a discussion on marginalization) the third column displays best fit of the marginalized posterior PDF for each parameter, finally, the fourth column describes the $68 \%$ limit of the marginalized posterior PDF mean for each parameter.

\begin{tabular}{cccc}
\hline \hline & Overall & \multicolumn{2}{c}{ Marginalized } \\
\cline { 2 - 4 } Parameter & Best-fit & Best-fit & $68 \%$ limits \\
\hline$\Omega_{\mathrm{m}}$ & 0.263 & 0.265 & $0.260_{-0.026}^{+0.025}$ \\
$f_{\mathrm{b}}$ & 0.154 & 0.167 & $0.148_{-0.031}^{+0.047}$ \\
$\sigma_{8}$ & 0.754 & 0.753 & $0.753_{-0.009}^{+0.010}$ \\
\hline \hline
\end{tabular}

Table 4.6: Results for the analysis of the Onion simulation APS of matter on a redshift shell with mean redshift 0.45 and width 0.1 . The second column labels the best fit of each parameter after taking the maximum of the overall posterior PDF, the third column displays best fit of the marginalized posterior PDF for each parameter and the fourth column describes the $68 \%$ limit of the marginalized posterior PDF mean for each parameter.

The figure 4.20 shows the marginalized posterior PDFs for each parameter considered. This figure also shows the contours of $68 \%$ and $95 \%$ confidence from the marginalized posterior PDFs for every pair of parameters. 


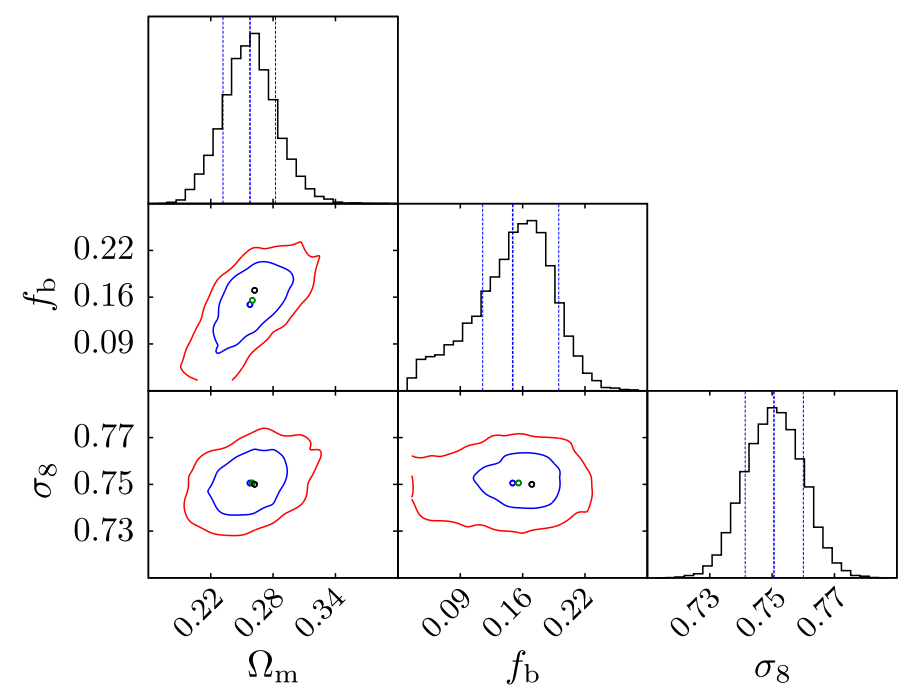

Figure 4.20: Posterior PDFs resulting from the analysis of the APS of matter in the Onion simulation in a redshift shell with mean redshift 0.45 and width 0.1 . The off-diagonal contour plots show the $68 \%(1 \sigma)$, blue lines, and $95 \%(2 \sigma)$, red lines, confidence regions for the two-parameters marginalized posterior PDFs. The circles at the center of each one of these plots show the marginalized mean (blue), marginalized best-fit (black) and overall best-fit (green) values for each parameter. The diagonal plots show the marginalized one-parameter posterior PDFs, the dashed blue lines show the marginalized mean values and $68 \%$ confidence regions. The numerical values of these results are quoted on table 4.6.

In figure 4.21 we show the theory prediction for the matter APS in a redshift shell with mean redshift 0.45 and width 0.1 for the Onion simulation together with the weak convergence APS for sources at $z=0.993$ and its cross correlation. The three APSs are evaluated at the Onion simulation cosmology, the PDF mean for each parameter, the best fit of each parameter after taking the maximum of the posterior PDF and the best fit of the marginalized posterior PDF for each parameter.

From this figure we can conclude that our modeling for the APS, as discussed on section 2.2 seems to do not reproduce the observed data. 

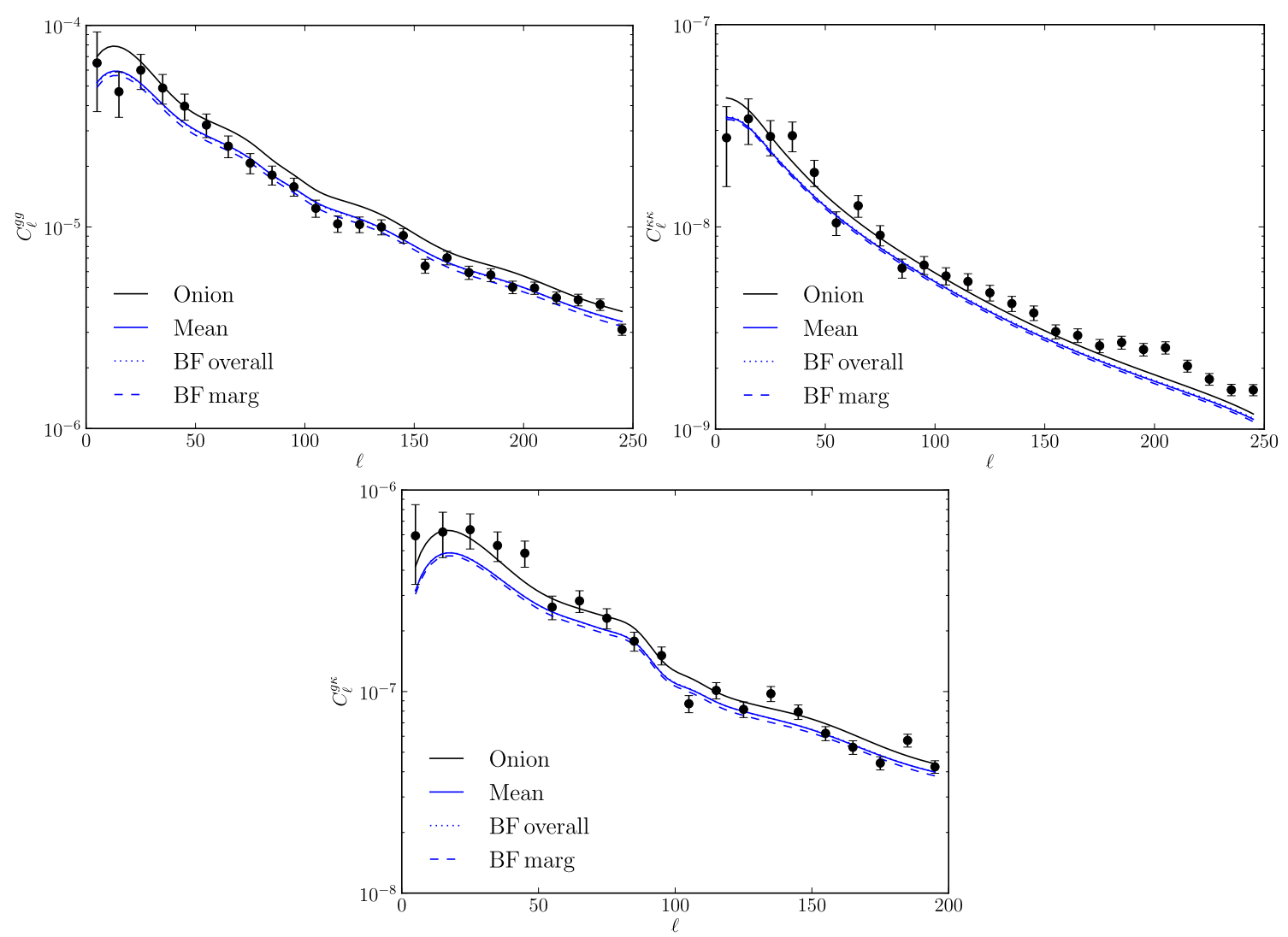

Figure 4.21: Top-left panel shows the matter APS in a redshift shell with mean redshift 0.45 and width 0.1 , top left weak lensing convergence APS for sources at $z=0.993$ and bottom panel the cross correlation between the in them. For each APS the black continuous line represents the Onion simulation cosmology, the blue continuous line represents the posterior PDF mean for each parameter, the blue dotted line the best fit of each parameter after taking the maximum of the posterior PDF and the dashed blue line the best fit of the marginalized posterior PDF for each parameter.

We stress that our results are quite preliminary at this point. We found results that are consistent up to $1 \sigma$ for $\Omega_{\mathrm{m}}$ and $f_{\mathrm{b}}$ with the original Onion Simulation parameters. However, for the amplitude of perturbations, measured in $\sigma_{8}$ the results are inconsistent up to $1 \sigma$. This inconsistency is in fact related to the fact that, as can be seen from figure 4.21 the normalization of the measured spectra disagree with the normalization of the modeled spectra computed on the Onion cosmology. We argue that this inconsistency should be related with our method of measurement of the Angular Power Spectra.

We hope to repeat these analysis to obtain a better understanding of this issues after developing a better estimation technique for the APS. After this step was completed, we would like to add to our analysis all the features included on the analysis presented for the ACF, physical effects as RSD and nonlinearities. A major goal for the future is also to properly implement the combination of these tree APSs. As previously stated the combination is needed in order to improble the contraints in the context of Bayesian inference. 


\section{Chapter 5}

\section{Conclusions and outlook}

The advent of wide-area photometric galaxy surveys brings new practical and theoretical challenges in cosmological analysis. These surveys will gain in area and depth, in exchange for a poorer determination of radial positions and have been planned to focus on the measure of four sets of cosmological observables, in order to study mainly the problem of cosmic acceleration. These probes are (a) the large scale distribution of galaxies, (b) the weak lensing shear induced on their shapes, (c) the abundance of galaxy clusters and (d) Type Ia supernovae (see e.g. [1]). From the first two of these observables, three types of two-point correlations can be constructed: the angular correlations between the positions of the foreground galaxies, the correlations of the lensing shear/convergence between background galaxies, and the galaxy-shear/convergence cross correlations induced by the association of dark matter with foreground galaxies.

In this context, and considering the great success of the Standard Cosmological Model (the concordance $\Lambda \mathrm{CDM}$ model) in describing most of the cosmological observations up to date, in principle, one can describe most statistical properties of cosmological structure observables in terms of a single quantity: the power spectrum of total mass fluctuations in the Universe. In fact, within this theoretical framework, structure in the Universe originates from nearly Gaussian random density fluctuations in the initial conditions and its evolution depends on the energy/matter content according to standard GR, which imply its dependence on the properties of the dark energy and dark matter. An important remaining task in order to extract dark-sector information from cosmological structures is the determination of the relationship between observables and the underlying mass power spectrum.

In section 1.4 a revision of the theoretical interpretation of angular galaxy clustering and weak lensing convergence as main cosmological observables in the context of widearea photometric galaxy surveys was presented. The philosophy of the approach presented here was to develop these concepts in the most general scenario possible theoretically.

The statistical interpretation of the cosmic fields allows us to connect theoretical interpretations with large-scale measurements in the Universe. The fundamental hypothesis of statistical homogeneity and isotropy implies the equivalence between statistics in both configuration and harmonic spaces. In sections 2.1 and 2.2 a review of basic statistical tools in configuration and harmonic space was presented, and the general definition of the power spectrum of a statistically homogeneous and isotropic random field was given. A general description for angular power spectra that comes from fields on the unit sphere (sky) as the result of projection of a 3D cosmological field was also discussed, which allowed for the introduction of auto- and cross- correlations between statistical fields on the 
sphere.

The increasing amount and quality of information from large galaxy redshift surveys demands models capable of describing the clustering of the galaxy distribution with high accuracy. Two-point statistic correlators of the matter content in the Universe constitute the most commonly used tools to analyze galaxy clustering, and their shape, both in configuration and harmonic spaces, is distorted by non-trivial effects such as non-linear dynamics, bias and redshift-space distortions. Moreover, astrophysical issues related with the observations themselves must be included in data analyses as systematics for the different cosmological observable measurements. These effects complicate the relation between the observations and the predictions of cosmological perturbation theory at linear level, making the interpretation of these measurements in terms of constraints on cosmological parameters more difficult.

In $\S 4.1 .1$ a general theory for the redshift-space two point correlation function in FLRW universes was presented based on the early work of [35]. It accounts simultaneously for wide-angle effects and cosmological redshift-space distortions. The final result is a general formula applicable for any pair of points in our past light-cone with arbitrary angular separation between the lines of sight, arbitrary redshifts and for any of the three different spatial geometries allowed in FLRW models. This general expression may be contrasted and compared to the commonly used formula, which is only valid under the assumptions of a spatially flat background and the distant observer approximation. The proper interpretation of these two assumptions was then given in light of the general formula as proper limiting cases.

In the context of the general treatment of the two-point correlation function, a review the modeling of the angular correlation function of galaxies (in configuration space) accounting for linear redshift-space distortions, photometric redshift errors, non-linear gravitational dynamics and local linear bias was presented. Finally, under the assumptions of a flat FLRW background and the distant observer approximation the results present on the literature $[7,92]$ were recovered.

In $\S 4.1 .4$ an analysis of the large-scale ACF of luminous galaxies from SDSS-DR8 photometric data was presented. The ACF was measured in four photo- $z$ bins with the novel approach developed by $[69,86]$, which makes an effort to incorporate systematics effects as stellar contamination and masking, galactic extinction, airmass and seeing, succeeding in the challenge of removing the excess of power at large scales reported by previous photometric studies [82, 83].

A cosmological analysis of the CMASS luminous galaxies, a photometric-redshift catalogue based on the Data Release 8 of the Sloan Digital Sky Survey-III with the ACF full shape information was presented accounting for the correlation between redshift shells and effects of photo- $z$ errors encoded in the selection function. This represents, to the best of our knowledge, the first cosmological analysis performed with the ACF in configuration space and has been published in [12]. Constraints in $\Omega_{\mathrm{m}}$ and $f_{\mathrm{b}}$ were found performing a Bayesian inference analysis with the information contained on each redshift bin independently. It was found that the best-fit values oscillate around the WMAP7 values, but are all consistent within $2 \sigma$. When combining the information contained on all the redshift bins, with the full covariance matrix accounting for the redshift correlations, the constraints found were: $\Omega_{\mathrm{m}}=0.280 \pm 0.022$ and $f_{\mathrm{b}}=0.211 \pm 0.026$ in reasonable agreement with WMAP7. Perhaps the most important conclusion taken from this work was the fact that it was possible to demonstrate that the ACF estimated from photometric data can be efficiently applied to constrain cosmological parameters. The ACF results 
for the photometric SDSS-III DR8 data are clearly not as competitive as those from the spatial correlation function, which already provides stronger constraints with the BOSS DR9 data [97]. Nonetheless, the results presented are encouraging for future photometric galaxy surveys, such as the DES, PanSTARRS and LSST, which will probe larger redshifts and measure significantly more galaxies. In this case, the ACF measurements have the potential to accurately constrain a larger number of cosmological parameters [7], allowing for extra consistency checks with other independent probes.

In $\S 4.2$, as a very naive application of the statistical methods in harmonic space, measures of the galaxy-galaxy (position), convergence-convergence (lensing) and galaxyconvergence (position-lensing) angular power spectra from the Onion Universe Simulations were presented. The measurements were performed for a redshift bin of foreground dark matter particles at $\hat{z}=0.45$ with $\Delta z=0.1$ homogeneously distributed in redshift and source or background galaxies at $z_{s}=0.993$, also homogeneously distributed in redshift. As a simple exercise we applied a Bayesian inference MCMC method only on the angular power spectrum of galaxies assuming Gaussian errors. Our results were summarized in §4.2.3. Our major inconsistency was on the $\sigma_{8}$ constraint, which was incompatible with the actual Onion Simulation value. We argue that this result may be due to the oversimplified method used on the measurements of the spectra and for not taking into account properly non-linear gravitational effects on the theoretical modelling.

As future lines of research, we would like to extend our analysis of weak lensing observables in harmonic space to configuration space, i.e. we will compare the results obtained with both the angular power spectra and the angular correlation functions, not only of convergence but also of the shear field, its decomposition on $E / B$ modes and the aperture mass statistics [11]. We would also like to investigate in detail the full impact of the assumptions of the distant observer approximation and spatial flatness that support usual analyses of the two-point correlation function in photometric galaxy surveys. 
Appendices 



\section{Appendix A}

\section{Cosmological perturbations}

The present Appendix is intended to review cosmological perturbation theory. We begin by reviewing some results for the background FLRW spacetime needed for some developments presented on the dissertation. Then, the gauge problem is discussed and gauge invariant first order EFE for a pure dust fluid under scalar perturbations are considered. The latter constitutes the basic theoretical basis to understand the large scale structure of the Universe within GR in the linear regime.

\section{A.1 Background Geometry}

In this section we present some geometrical quantities for the background FLRW Universe relevant for the development of the present work. In particular we are interested on the connection coefficients (Christoffel symbols) for different chooses of comoving spatial system of coordinates considered on $\S 1.1$.

Starting from the RW metric, equation (1.3), one can derive the Christoffel symbols of the metric connection from its definition $[14,2]$,

$$
\bar{\Gamma}_{\nu \sigma}^{\mu}=\frac{1}{2} \bar{g}^{\mu \tau}\left(-\partial_{\tau} \bar{g}_{\nu \sigma}+\partial_{\nu} \bar{g}_{\tau \sigma}+\partial_{\sigma} \bar{g}_{\nu \tau}\right)
$$

as

$$
\bar{\Gamma}^{0}{ }_{00}=\mathcal{H}, \quad \bar{\Gamma}^{0}{ }_{i j}=\mathcal{H} \gamma_{i j}, \quad \bar{\Gamma}^{i}{ }_{0 j}=\bar{\Gamma}^{i}{ }_{j 0}=\mathcal{H} \gamma_{j}^{i}, \quad \bar{\Gamma}^{i}{ }_{j k}={ }^{3} \Gamma^{i}{ }_{j k},
$$

where ${ }^{3} \Gamma^{i}{ }_{j k}$ denotes the Christoffel symbols associated to the spatial metric $\gamma$. On this work the forms (1.4) and (1.6) are considered. The non-null symbols for this chooses of coordinates are

$$
\begin{aligned}
\bar{\Gamma}_{12}^{2}=\bar{\Gamma}^{3}{ }_{13}= & \left\{\begin{array}{ll}
\cosh (\chi) / \sinh (\chi) & K<0, \\
\chi^{-1} & K=0, \\
\cos (\chi) / \sin (\chi) & K>0,
\end{array} \quad \bar{\Gamma}_{33}^{2}=-\cos (\theta) \sin (\theta),\right. \\
\bar{\Gamma}^{1}{ }_{33} & =\left\{\begin{array}{ll}
-\cosh (\chi) \sinh (\chi) \sin ^{2}(\theta) & K<0, \\
-\chi \sin ^{2}(\theta) & K=0, \\
-\cos (\chi) \sin (\chi) \sin ^{2}(\theta) & K>0,
\end{array} \quad \bar{\Gamma}_{23}^{3}=\frac{\cos (\theta)}{\sin (\theta)},\right. \\
\bar{\Gamma}_{22}^{1} & = \begin{cases}-\cosh (\chi) \sinh (\chi) & K<0, \\
-\chi & K=0, \\
-\cos (\chi) \sin (\chi) & K>0,\end{cases}
\end{aligned}
$$


for $x^{i}=(\chi, \theta, \varphi)$, equation (1.4), and

$$
\begin{aligned}
\bar{\Gamma}_{12}^{2}= & \bar{\Gamma}^{3}{ }_{13}=f_{K}^{\prime}(r) / f_{K}(r), \\
\bar{\Gamma}_{33}^{1} & =-f_{K}(r) f_{K}^{\prime}(r) \sin ^{2}(\theta), \\
\bar{\Gamma}_{33}^{2} & =-\cos (\theta) \sin (\theta),
\end{aligned}
$$

where the $f_{K}$ is given by equation $(1.7)$, for $x^{i}=(r, \theta, \varphi)$, equation (1.6).

\section{A.2 Perturbation theory and the gauge problem}

In perturbation theory the main goal is to find approximated solutions to Einstein field equations which can be considered as small deviations from a known exact solution, the background spacetime solution. The perturbation of any tensorial field $\mathbb{T}$ should then be given by the difference between its value in the physical spacetime $\mathbb{T}$ and its corresponding value on the background spacetime $\overline{\mathbb{T}}$. However, its a basic fact of in differential geometry that in order to perform comparisons of such quantities one should consider them at the same point on a manifold. Since the manifolds representing the physical and background spacetimes are different the necessity for a prescription to identify events between them emerges. Such a prescription is what should be understood as a gauge choice, see [23, 25, 2] and references therein.

One then ends with a definition of gauge choice as a diffeomorphism between the background and physical spacetimes. Different choses for this diffeomorphism and its relations should define gauge transformations and the freedom to choose it is equivalent to the freedom to chose the functional forms for the perturbation of any tensor field. This statement constitutes a framework to specify the gauge problem.

A way to put on formal grounds the previous ideas is to introduce a family of differential manifolds as models of the spacetime $\left\{\left(M_{\lambda}, g_{\lambda}\right) \mid \lambda \in \Lambda\right\}$, here $M_{\lambda}$ defines its topological structure and $g_{\lambda}$ represents its associated metric, see $\S 7.5$ of [22]. There is a natural way to treat this situation, by introducing a $(4+1)$-dimensional manifold $N$ defined as the product manifold $N=M \times \mathbb{R}$, where each $M_{\lambda}$ is a 4 -dimensional manifold, $M_{\lambda}=\mathbb{M} \times \lambda$, so that $N$ can be interpreted as a foliation, $N=\cup_{\lambda \in \mathbb{R}} M_{\lambda}$. This formulation is clearly explained in [25]. Here we present a review of the main concepts and ideas in presented there in order to obtain the perturbed EFE in first order gauge invariant variables.

Since the natural differential structure over $\mathbb{R}$ consist of charts $(U, i d)^{1}$, there is a natural form for the chart over $N$ formed by charts $\left(U, h_{U}\right)$ around $q=(p, \lambda) \in N^{2}$ such that $h(q)=\left(x^{0}, x^{1}, x^{2}, x^{3}, \lambda\right) \in \mathbb{R}^{5}$, where the coordinates $x^{a}$ with $a=0,1,2,3$ are the ones associated to the local chart on $M$ around $p$.

If a tensorial field $\mathbb{T}_{\lambda}$ is defined on each model of spacetime $M_{\lambda}$ a tensorial field is defined on $N$. In fact,

$$
\forall q=(p, \lambda) \in N, \quad \mathbb{T}_{q}=\mathbb{T}_{(p, \lambda)}:=\mathbb{T}_{\lambda}(p) \quad\left(p \in M_{\lambda}\right) .
$$

Particularly, on each spacetime model a metric tensor and a set of matter and radiation fields exist satisfying the Einstein field equations.

In order to formally define a perturbation of a tensorial field, as was previously discussed, the basic requirement is a way to compare $\mathbb{T}_{\lambda}$ with $\mathbb{T}_{0}:=\overline{\mathbb{T}}$ for every $\lambda$. This

\footnotetext{
${ }^{1}$ Here $U$ is an open set on $\mathbb{R}$ and $i d$ represents the identity application.

${ }^{2}$ Here $p \in M$ and $\lambda \in \mathbb{R}$.
} 
is equivalent to define a diffeomorphism between each model $M_{\lambda}$ and the background spacetime $\bar{M}:=M_{0}$. Therefore, one can consider all the diffeomorphisms $\varphi_{\lambda}: N \rightarrow N$ such that its restriction to $M_{0}$ cover $M_{\lambda}$, i.e., $\left.\varphi_{\lambda}\right|_{M_{0}}: M_{0} \rightarrow M_{\lambda}$ is a diffeomorphism. By choosing each $\varphi_{\lambda}$ as a member of the group of diffeomorphisms associated to the flux of a vectorial field over $N$ one can instead to deal with the diffeomorphism itself to deal with the field whose flux over $N$ is given by $\varphi_{\lambda}$. The condition $\left.\varphi_{\lambda}\right|_{M_{0}}: M_{0} \rightarrow M_{\lambda}$ for the diffeomorphism translates to the field as the condition that on the natural charts for $N$ discussed above the fifth component of the field should be the unity.

Summarizing, one can define a gauge vector field as a vector field $\mathbf{X}$ over $N=M \times$ $\mathbb{R}$ such that on the differential structure induced by the natural differential structure on $\mathbb{R}$ have the property $X^{4}=1$ for each $q \in N$. That is because such fields define trough its fluxes over $N$ the way to identify events on the spacetime models $M_{\lambda}$ and $M_{0}$ diffeomorphically.

\section{A.2.1 Taylor expansion of a tensor field}

A Taylor expansion is a convenient way to write the value of a function on some given point of its domain in terms of its value and the value of all its derivatives on some other near point. This is impossible for a tensor field defined on a manifold just because its value at different points on the manifold, $\mathbb{T}_{q}$ and $\mathbb{T}_{q^{\prime}}$ for $q \neq q^{\prime} \in N$, are objects belonging to different spaces by definition. However, a Taylor-like expansion can be defined when an application between tensors on different points of a manifold is given, and in particular, when this application is viewed as induced by the flux of a vector field on the manifold.

Given $\varphi^{\mathbf{X}}: \mathbb{R} \times N \rightarrow N$, the flux of a vector field $\mathbf{X}$ over $N, q \in N$ and $\mathbb{T}$ a tensor field on $N$. Since for every $\lambda \in \mathbb{R}, \varphi_{\lambda}^{\mathbf{X}}$ defines a diffeomorphism of $N$ onto $N$, the pull-back of $\mathbb{T}_{\varphi_{\lambda}^{\mathbf{X}}(q)}$ trough $\varphi_{\lambda}^{\mathbf{X}}$ (the tensor field $\mathbb{T}$ evaluated at $\left.\varphi_{\lambda}^{\mathbf{X}}(q) \in N\right), \varphi_{\lambda}^{\mathbf{X}} *\left(\mathbb{T}_{\varphi_{\lambda}^{\mathbf{X}}(q)}\right)^{3}$, defines a tensor evaluated at $q \in N$. Such a tensor admits the following Taylor expansion around $\lambda=0$, lemma 1 in [25]:

$$
\varphi_{\lambda}^{\mathbf{X} *}\left(\mathbb{T}_{\varphi_{\lambda}^{\mathbf{X}}(q)}\right)=\sum_{n=0}^{\infty} \frac{\lambda^{n}}{n !}\left(\mathcal{L}_{\mathbf{X}}^{n} \mathbb{T}\right)_{q},
$$

where $\left(\mathcal{L}_{\mathbf{X}} \mathbb{T}\right)_{q}$ denotes the tensor over $N$ given by the Lie derivative of $\mathbb{T}_{q}$ both at $q \in N$ and $\left(\mathcal{L}_{\mathbf{X}}^{n} \mathbb{T}\right)_{q}$ denotes the $n$-th Lie derivative under the same of $\mathbb{T}$ at $q$. For a good review of induced maps (pull-back and push-forward maps) and Lie derivatives see e.g. chapter 5 of [98].

The general Taylor expansion (A.6) allows to define the perturbation of a tensor field. Let $\mathrm{X}$ be a gauge field on the family of spacetime models $N=\mathbb{R} \times M$ and $\mathbb{T}$ a tensor field defined on each model and extended to $N$ according to (A.5), then the perturbation of $\mathbb{T}$ can be defined as a tensor field on the background spacetime $\bar{M}$ as

$$
\delta_{\mathbf{X}}^{(n)}[\mathbb{T}]:=\left.\mathcal{L}_{\mathbf{X}}^{n} \mathbb{T}\right|_{\bar{M}}
$$

in such a way that, according to (A.6), the tensor field associated with the tensor in any model of spacetime, since is given in the background by the pullback of $\varphi_{\lambda}^{\mathbf{X}}$, can be written as

$$
\varphi_{\lambda}^{\mathbf{X} *}(\mathbb{T})=\overline{\mathbb{T}}+\sum_{n=1}^{\infty} \frac{\lambda^{n}}{n !} \delta_{\mathbf{X}}^{(n)}[\mathbb{T}] .
$$

\footnotetext{
${ }^{3}$ Here the superscript ${ }^{*}$ denotes the pull-back induced mapping.
} 
Note that the perturbations of $\mathbb{T}, \delta_{\mathbf{X}}^{(n)}[\mathbb{T}]$, are tensor fields on the background, this formalizes the common statement in the literature that perturbations are tensor fields defined on the background. The parameter $\lambda$ used to label different models of spacetime also works as perturbative parameter according to the gauge field definition.

\section{A.2.2 Gauge transformations and gauge invariance}

The natural question that remains from the previous construction is how the perturbation of a tensor fields changes under a change of gauge.

Consider two gauge fields $\mathbf{X}$ and $\mathbf{Y}$ generating fluxes $\varphi_{\lambda}^{\mathbf{X}}$ and $\psi_{\lambda}^{\mathbf{Y}}$ over $N$, respectively. This two fields, through the pullback of its fluxes, allow to define two background representations of the same tensor field $\mathbb{T}, \varphi_{\lambda}^{\mathbf{X} *}(\mathbb{T})$ and $\psi_{\lambda}^{\mathbf{Y} *}(\mathbb{T})$, and thus two perturbative expansions of the form of (A.8).

Define a family of diffeomorphisms

$$
\Psi_{\lambda}:=\varphi_{\lambda}^{\mathbf{X}} \circ \psi_{\lambda}^{\mathbf{Y}}: N \rightarrow N \quad(\forall \lambda \in \mathbb{R}) .
$$

Then this family satisfies the following properties: (a) for each $\left.\lambda \in \mathbb{R} \Psi_{\lambda}\right|_{\bar{M}}$ defines a diffeomorphism of $\bar{M}$ onto itself, (b) $\left\{\Psi_{\lambda} \mid \lambda \in \mathbb{R}\right\}$ is not an uniparametric group and (c) for each tensor field $\mathbb{T}$

$$
\psi_{\lambda}^{\mathbf{Y} *}(\mathbb{T})=\Psi_{\lambda}^{*}\left(\varphi_{\lambda}^{\mathbf{X} *}(\mathbb{T})\right) .
$$

Properties (a) and (b) follow from the construction of family (A.9), while property (c) follows from the property of pullbacks $(\varphi \circ \psi)^{*}=\psi^{*} \circ \varphi^{*}$, and allow to formally connect the two background representations of any tensor field, thus defining a gauge transformation from $\mathbf{X}$ to $\mathbf{Y}$.

Having formalized the idea of gauge transformation is convenient to introduce the concept of gauge invariant tensor field. Given $\mathbf{X}$ and $\mathbf{Y}$ gauge fields, a tensor field $\mathbb{T}$ is said to be totally invariant if its background representations on both gauges coincide, i.e., if $\varphi_{\lambda}^{\mathbf{X} *}(\mathbb{T})=\psi_{\lambda}^{\mathbf{Y} *}(\mathbb{T})$.

On any practical application one is interested on perturbations up to some given order, so that it is convenient to have a more relax definition of gauge invariance up to some order. A tensor field $\mathbb{T}$ is said to be gauge invariant up to order $n$ if all its perturbations on both gauges, up to order $n$ coincide, i.e., if

$$
(\forall k \leq n) \quad \delta_{\mathbf{X}}^{(k)}[\mathbb{T}]=\delta_{\mathbf{Y}}^{(k)}[\mathbb{T}]
$$

For $n \geq 1$ the condition for a tensor field to be gauge invariant up to order $n$, equation (A.11), is equivalent to say that for every vector field $\mathbf{V}$ on $\bar{M}$ and for all $k \leq n$,

$$
\mathcal{L}_{\mathbf{V}} \delta_{\mathbf{X}}^{(k)}[\mathbb{T}]=0
$$

This result can be probed by induction over $n$. For $n=1$, follows from the definition of the perturbations, equation (A.7), that

$$
\delta_{\mathbf{X}}^{(1)}[\mathbb{T}]=\left.\delta_{\mathbf{Y}}^{(1)}[\mathbb{T}] \quad \Leftrightarrow \quad \mathcal{L}_{\mathbf{V}} \mathbb{T}\right|_{\bar{M}}=0
$$

so that, since $\mathbf{X}$ and $\mathbf{Y}$ define arbitrary vector fields on $\bar{M}$ the field $\mathbf{V}:=\mathbf{X}-\mathbf{Y}$ is an arbitrary vector field on $\bar{M}$. By supposing (A.12) is true for some $n>1$ then from the definition of perturbations,

$$
\delta_{\mathbf{X}}^{(n+1)}[\mathbb{T}]=\left.\delta_{\mathbf{Y}}^{(n+1)}[\mathbb{T}] \quad \Leftrightarrow \quad \mathcal{L}_{\mathbf{V}} \delta_{\mathbf{X}}^{(n)}[\mathbb{T}]\right|_{\bar{M}}=0
$$


This probes the result.

The result (A.12) is a generalization of the Stewart-Walker lemma [99]. Defining a useful way to probe for a tensor degree of freedom to be gauge invariant.

Although the result (A.12) answers to the question of how to determine whether a tensor field is gauge invariant up to some order, the question of how do change this perturbations under gauge transformations, equation (A.10), remains open.

In order to consider this problem, one have to introduce the concept of Knight diffeomorphism [25]. Let $\left\{\mathbf{V}_{(n)} \mid n \in \mathbb{N}\right\}$ a non-numerable family of vector fields over $N$ and

$$
\left\{\phi_{\lambda}^{(n)}:=\varphi_{\lambda}^{\mathbf{V}_{(n)}}: \mathbb{R} \times N \rightarrow N \mid n \in \mathbb{N}\right\}
$$

the family of its corresponding generated fluxes. A uniparametric family of Knight diffeomorphisms is defined to be formed by the applications

$$
\begin{aligned}
\Psi_{\lambda}: \mathbb{R} \times N & \rightarrow N \\
(\lambda, p) & \mapsto \Psi_{\lambda}(p):=\phi_{\lambda}^{(1)} \circ \phi_{\frac{\lambda^{2}}{2}}^{(2)} \circ \cdots \circ \phi_{\frac{\lambda^{n}}{n !}}^{(n)} \circ \cdots(q) .
\end{aligned}
$$

That is, a Knight diffeomorphism over $N$ is a suitable composition of the fluxes of a non-numerable family of vector fields over $N$. The vector fields $\mathbf{V}_{(1)}, \mathbf{V}_{(2)}, \ldots$ are called generators of the Knight diffeomorphism $\Psi_{\lambda}$. It can be probed that in general a family of Knight diffeomorphisms (A.15) does not form a uniparametric group of diffeomorphisms.

Knight diffeomorphisms has two important properties: (a) the pullback of a tensor field $\mathbb{T}$ on $N$ induced by a uniparametric family of Knight diffeomorphisms with gerenators $\left\{\mathbf{V}_{(n)} \mid n \in \mathbb{N}\right\}$ can be expanded around $\lambda=0$ as, lemma 2 in [25]:

$$
\Psi_{\lambda}^{*}\left(\mathbb{T}_{\Psi_{\lambda}(q)}\right)=\sum_{\ell_{1}=0}^{\infty} \sum_{\ell_{2}=0}^{\infty} \cdots \sum_{\ell_{n}=0}^{\infty} \cdots\left[\prod_{n=1}^{\infty} \frac{1}{\ell_{n} !}\left(\frac{\lambda^{n}}{n !}\right)^{\ell_{n}}\right]\left(\cdots \mathcal{L}_{\mathbf{V}_{(n)}^{\ell_{n}}} \cdots \mathcal{L}_{\mathbf{V}_{(2)}}^{\ell_{2}} \mathcal{L}_{\mathbf{V}_{(1)}}^{\ell_{1}} \mathbb{T}_{q}\right),
$$

$\forall q \in N$. The proof of this property follows from the expansion for the pullback of the flux of a vector field on a tensor $\mathbb{T}$, equation (A.6). In fact, given $q \in N$

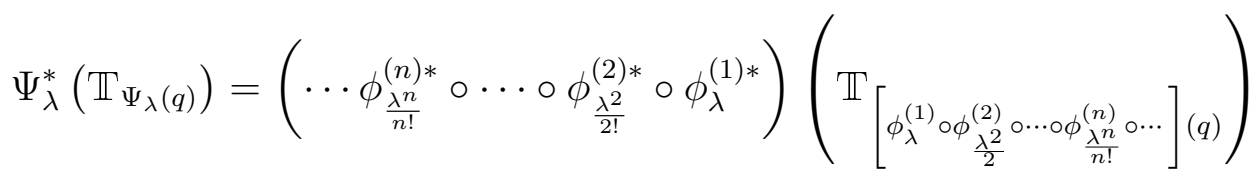

$$
\begin{aligned}
& =\left(\cdots \phi_{\frac{\lambda^{n}}{n !}}^{(n) *} \circ \cdots \circ \phi_{\frac{\lambda^{3}}{3 !}}^{(3) *} \circ \phi_{\frac{\lambda^{2}}{2 !}}^{(2) *}\right) \sum_{\ell_{1}=0}^{\infty} \frac{\lambda^{\ell_{1}}}{\ell_{1} !}\left(\mathcal{L}_{\mathbf{V}_{(1)}}^{\ell_{1}} \mathbb{T}\right) \sum_{\left.\phi_{\frac{\lambda^{2}}{2 !}}^{(2)} \circ \phi_{\frac{\lambda^{3}}{3 !}}^{(3)} \circ \cdots \circ \phi_{\frac{\lambda^{n}}{n !}}^{(n)} \circ \cdots\right](q)} \\
& =\left(\cdots \phi_{\frac{\lambda^{n}}{n !}}^{(n) *} \circ \cdots \circ \phi_{\frac{\lambda^{4}}{4 !}}^{(4) *} \circ \phi_{\frac{\lambda^{3}}{3 !}}^{(3) *}\right) \sum_{\ell_{2}=0}^{\infty} \frac{\lambda^{2 \ell_{2}}}{(2 !)^{\ell_{2} \ell_{2} !}}
\end{aligned}
$$

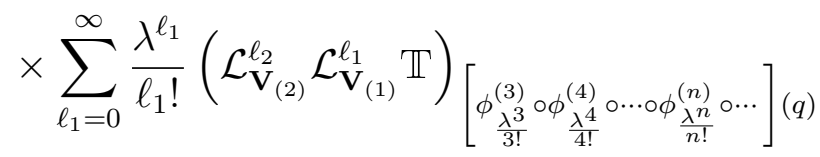

$$
\begin{aligned}
& =\sum_{\ell_{1}=0}^{\infty} \sum_{\ell_{2}=0}^{\infty} \cdots \sum_{\ell_{n}=0}^{\infty} \cdots\left[\frac{\lambda^{\ell_{1}} \lambda^{2 \ell_{2}} \cdots \lambda^{n \ell_{n}} \cdots}{(2 !)^{\ell_{2}}(3 !)^{\ell_{3}} \cdots(n !)^{\ell_{n}} \cdots \ell_{1} \ell_{2} \cdots \ell_{n} \cdots}\right] \\
& \times\left(\cdots \mathcal{L}_{\mathbf{V}_{(n)}}^{\ell_{n}} \cdots \mathcal{L}_{\mathbf{V}_{(2)}}^{\ell_{2}} \mathcal{L}_{\mathbf{V}_{(1)}}^{\ell_{1}}\right)_{q},
\end{aligned}
$$


which reduces to (A.16); and (b) given $\Psi: \mathbb{R} \times N \rightarrow N$ an uniparametric family of diffeomorphisms over $N$, exist $\phi^{(1)}, \ldots \phi^{(2)}, \ldots, \phi^{(n)}, \ldots$ uniparametric groups of diffeomorphisms over $N$, such that each diffeomorphism $\Psi_{\lambda}$ can be written as the composition

$$
\Psi_{\lambda}=\phi_{\lambda}^{(1)} \circ \phi_{\frac{\lambda^{2}}{2}}^{(2)} \circ \cdots \circ \phi_{\frac{\lambda^{n}}{n !}}^{(n)} \circ \cdots
$$

in other words, every uniparametric family of diffeomorphisms $\Psi_{\lambda}$ can always be written as a family of Knight diffeomorphisms, theorem 2 in [25].

To see a little bit better how the expansion (A.16) works, one can consider it explicitly up to second order,

$$
\Psi_{\lambda}^{*}\left(\mathbb{T}_{\Psi_{\lambda}(q)}\right) \approx \mathbb{T}_{q}+\lambda\left(\mathcal{L}_{\mathbf{V}_{(1)}} \mathbb{T}\right)_{q}+\frac{\lambda^{2}}{2 !}\left(\mathcal{L}_{\mathbf{V}_{(1)}}^{2} \mathbb{T}+\mathcal{L}_{\mathbf{V}_{(2)}} \mathbb{T}\right)_{q}+\cdots
$$

Properties (a) and (b) combined allow to treat the gauge transformation law $\mathbf{X} \rightarrow \mathbf{Y}$ given by the uniparametric family of diffeomorphisms

$$
\Psi_{\lambda}=\varphi_{-\lambda}^{\mathbf{X}} \circ \psi_{\lambda}^{\mathbf{Y}}: \bar{M} \rightarrow \bar{M}
$$

according to equation (A.10) as a Knight diffeomorphism. Therefore, given $\mathbb{T}$ a tensor field on $M$, by comparing the expansions of the expression (A.10) using (A.6) and (A.16) (or (A.18) to go up to second order only) one can determine the relations between its perturbation on two different gauges $(\mathbf{X}$ and $\mathbf{Y})$ up to second order as

$$
\begin{aligned}
& \delta_{\mathbf{Y}}^{(1)}[\mathbb{T}]-\delta_{\mathbf{X}}^{(1)}[\mathbb{T}]=\mathcal{L}_{\mathbf{V}_{(1)}} \mathbb{T}, \\
& \delta_{\mathbf{Y}}^{(2)}[\mathbb{T}]-\delta_{\mathbf{X}}^{(2)}[\mathbb{T}]=\mathcal{L}_{\mathbf{V}_{(2)}} \mathbb{T}+\mathcal{L}_{\mathbf{V}_{(2)}}^{2} \mathbb{T}+2 \mathcal{L}_{\mathbf{V}_{(1)}} \delta_{\mathbf{X}}^{(1)}[\mathbb{T}]
\end{aligned}
$$

where the $\mathbf{V}_{(n)}$ are the vector generators of $\Psi_{\lambda}$ seen as a Knight diffeomorphism given according to the perturbation definiton, equation (A.7), by (proposition 3 in [25])

$$
\begin{aligned}
\mathbf{V}_{(1)} & =\mathbf{Y}-\mathbf{X}, \\
\mathbf{V}_{(2)} & =[\mathbf{Y}, \mathbf{X}] .
\end{aligned}
$$

\section{A.3 First order perturbations}

\section{A.3.1 Scalar-vector-tensor decomposition}

Scalar-vector-tensor decomposition principle establish that for a three-dimensional differential manifold equipped with a metric tensor $\gamma$, and a covariant derivative $D$, every vector field $\mathbf{X}$ can be decomposed in a unique manner on a scalar mode $S$ and one transverse vector mode $\mathbf{V}$, that is, the components of $\mathbf{X}$ are given as

$$
X^{i}=D^{i} S+V^{i} ; \quad D^{i} V_{i}=0 .
$$

Here, $\mathbf{X}$ has three degrees of freedom (its three components) which are distributed as one on the scalar mode, and two on the vector mode, because of the transversal condition. 
Analogously, every second rank symmetric tensor $\mathbb{X}$ can be decomposed in a unique manner on two scalar modes $S_{1}$ and $S_{2}$, one transverse vector mode $\mathbf{V}$ and a symmetric, transverse and trace-free tensor mode $\mathbb{T}$ as

$$
X_{i j}=D_{i} D_{j} S_{1}+S_{2} \gamma_{i j}+D_{(i} V_{j)}+T_{i j} ; \quad D^{i} V_{i}=0, \quad D^{i} T_{i j}=\gamma_{i j} T^{i j}=0 .
$$

Here the six $\mathbb{T}$ degrees of freedom are distributed in one on each scalar mode, two on the vector mode, because of the transverse condition, and the remaining four on the tensor mode, because of the transverse and trace-free conditions.

A very common practice in the literature is to redistribute the scalar degrees of freedom into the trace of $\mathbb{X}$ and $S_{1}$, i.e., to change $S_{2}$ for the trace

$$
X=\gamma_{i j} X^{i j}=\nabla^{2} S_{1}+3 S_{2}
$$

where $\nabla^{2}$ is the Laplace operator on the three-dimensional manifold, $\nabla^{2}:=\gamma_{i j} D^{i} D^{j}$. In terms of the trace $X$ the decomposition of a symmetric second rank tensor, equation (A.23), is given by

$$
X_{i j}=\frac{1}{3} \gamma_{i j} X+\Delta_{i j} S_{1}+D_{(i} V_{j)}+T_{i j} ; \quad D^{i} V_{i}=0, \quad D^{i} T_{i j}=\gamma_{i j} T^{i j}=0,
$$

where the trace-free differential operator $\Delta$ was introduced as

$$
\Delta_{i j}=D_{i} D_{j}-\frac{1}{3} \gamma_{i j} \nabla^{2}
$$

The most important property of the decompositions (A.22) and (A.23) or (A.25) is its uniqueness. It is not the propose here to discuss about the conditions for this property to be satisfied, the interested reader can see e.g. [23].

\section{A.3.2 Metric perturbations}

Consider a gauge field $\mathbf{X}$. The most general way to parametrize the $n$-th order perturbation of the metric tensor around an FLRW background (in conformal time), on the light of scalar-vector-tensor decomposition for fields over the constant time hypersurfaces, is given by

$$
\begin{aligned}
& \delta_{\mathbf{X}}^{(n)}[\mathrm{g}]=a^{2}(\eta) {\left[-2_{\mathbf{X}} \Phi^{(n)} \mathrm{d} \eta \otimes \mathrm{d} \eta+\left(D_{i \mathbf{X}} B^{(n)}+\mathbf{X}_{i} B_{i}^{(n)}\right)\left(\mathrm{d} \eta \otimes \mathrm{d} x^{i}+\mathrm{d} x^{i} \otimes \mathrm{d} \eta\right)\right.} \\
&\left.+\left(-2 \mathbf{X}^{(n)} \gamma_{i j}+D_{i} D_{j \mathbf{X}} E^{(n)}+D_{(i \mathbf{X}} E_{j)}^{(n)}+2_{\mathbf{X}} E_{i j}^{(n)}\right) \mathrm{d} x^{i} \otimes \mathrm{d} x^{j}\right]
\end{aligned}
$$

where the notation $\mathbf{x} \mathbb{T}^{(n)}:=\delta_{\mathbf{X}}^{(n)}[\mathbb{T}]$ was introduced for simplicity. On the metric perturbation (A.27) the temporal component (00) defines a scalar mode parametrized as $-2 \mathbf{X} \Phi^{(n)}$, the mixed components $(0 i)$ and $(i 0)$ defines a vectorial field on the constant time hypersurfaces expanded according to (A.22) into a scalar mode $\mathbf{x} B^{(n)}$ and a transverse vector mode $\mathbf{x} B_{i}^{(n)}$, finally, the spatial components $(i j)$ define a symmetric tensor on the constant time hypersurfaces decomposed according to (A.23) into two scalar modes ${ }_{\mathbf{x}} \Psi^{(n)}$ and ${ }_{\mathbf{x}} E^{(n)}$, one transverse vector mode $\mathbf{x} E_{i}^{(n)}$ and one symmetric, transverse and trace-free tensor mode $\mathbf{x} E_{i j}^{(n)}$. 


\section{A.3.3 First order scalar perturbations}

During this work only linear perturbation scalar modes are considered. Let $\mathbf{X}_{\mathrm{S}}$ represents the vector gauge field in wich the perturbations are purely scalar. The first order perturbation for the metric tensor according to (A.27) is given by

$$
\begin{aligned}
\delta_{\mathbf{X}_{\mathrm{S}}}^{(1)}[\mathrm{g}]=a^{2}\left[-2 \Phi \mathrm{d} \eta \otimes \mathrm{d} \eta+D_{i} B \mathrm{~d} \eta \otimes \mathrm{d} x^{i}+D_{i} B \mathrm{~d} x^{i} \otimes \mathrm{d} \eta\right. \\
\left.+2\left(D_{i} D_{j} E-\Psi \gamma_{i j}\right) \mathrm{d} x^{i} \otimes \mathrm{d} x^{j}\right]
\end{aligned}
$$

where for all the scalar modes $S$ the notation is relaxed via $S:=\mathbf{x}_{\mathbf{S}} S^{(1)}=\delta_{\mathbf{x}_{S}}^{(1)}[S]$.

The contravariant components of the first order perturbation to the metric tensor can be computed by considering the identity $g^{\mu \nu}=\bar{g}^{\mu \nu}-\bar{g}^{\mu \lambda}\left(g_{\lambda \sigma}-\bar{g}_{\lambda \sigma}\right) g^{\sigma \nu}$. By perturbing this relation up to first order one can find for a general gauge specified by $\mathbf{X}$ the relation

$$
\delta_{\mathbf{X}}^{(1)}\left[g^{\mu \nu}\right]=-\bar{g}^{\nu \rho} \bar{g}^{\mu \sigma} \delta_{\mathbf{X}}^{(1)}\left[g_{\sigma \rho}\right]
$$

Choosing $\mathbf{X}_{\mathrm{S}}$ gauge, one finds

$$
\begin{aligned}
& a^{2} \delta_{\mathbf{X}_{\mathrm{S}}}^{(1)}\left[g^{00}\right]=2 \Phi, \\
& a^{2} \delta_{\mathbf{X}_{\mathrm{S}}}^{(1)}\left[g^{0 i}\right]=a^{2} \delta_{\mathbf{X}_{\mathrm{S}}}^{(1)}\left[g^{i 0}\right]=D^{i} B, \\
& a^{2} \delta_{\mathbf{X}_{\mathrm{S}}}^{(1)}\left[g^{i j}\right]=2\left(\gamma^{i j} \Psi-D^{i} D^{j} E\right) \text {. }
\end{aligned}
$$

The perturbations to the Christoffel symbols of second kind, equation (A.1), can obtained by perturbing its definition directly,

$$
\begin{aligned}
\delta_{\mathbf{X}}^{(1)}\left[\Gamma_{\mu \nu}^{\alpha}\right]=\frac{1}{2} \bar{g}^{\alpha \beta}\left(-\partial_{\beta} \delta_{\mathbf{X}}^{(1)}\left[g_{\mu \nu}\right]+\partial_{\nu} \delta_{\mathbf{X}}^{(1)}\left[g_{\beta \mu}\right]+\partial_{\beta} \delta_{\mathbf{X}}^{(1)}\left[g_{\beta \nu}\right]\right) & \\
+ & \frac{1}{2} \delta_{\mathbf{X}}^{(1)}\left[g^{\alpha \beta}\right]\left(-\partial_{\beta} \bar{g}_{\mu \nu}+\partial_{\nu} \bar{g}_{\beta \mu}+\partial_{\beta} \bar{g}_{\beta \nu}\right)
\end{aligned}
$$

so that, on the $\mathbf{X}_{\mathrm{S}}$ gauge

$$
\begin{aligned}
\delta_{\mathbf{X}_{\mathrm{S}}}^{(1)}\left[\Gamma_{00}^{0}\right] & =\Phi^{\prime} \\
\delta_{\mathbf{X}_{\mathrm{S}}}^{(1)}\left[\Gamma^{0}{ }_{0 i}\right]=\delta_{\mathbf{X}_{\mathrm{S}}}^{(1)}\left[\Gamma^{0}{ }_{i 0}\right] & =D_{i}(\Phi+\mathcal{H} B), \\
\delta_{\mathbf{X}_{\mathrm{S}}}^{(1)}\left[\Gamma^{0}{ }_{i j}\right] & =-\left[2 \mathcal{H}(\Phi+\Psi)+\Psi^{\prime}\right] \gamma_{i j}-D_{i} D_{j}\left(B-2 \mathcal{H} E-E^{\prime}\right), \\
\delta_{\mathbf{X}_{\mathrm{S}}}^{(1)}\left[\Gamma^{i}{ }_{00}\right] & =D^{i}\left(\Phi+B^{\prime}+\mathcal{H} B\right), \\
\delta_{\mathbf{X}_{\mathrm{S}}}^{(1)}\left[\Gamma_{0 j}^{i}\right]=\delta_{\mathbf{X}_{\mathrm{S}}}^{(1)}\left[\Gamma^{i}{ }_{j 0}\right]= & -\Psi^{\prime} \delta^{i}{ }_{j}+D^{i} D_{j} E^{\prime}, \\
\delta_{\mathbf{X}_{\mathrm{S}}}^{(1)}\left[\Gamma^{i}{ }_{j k}\right]= & \gamma_{j k} D^{i} \Psi-\gamma_{k}^{i} D_{j} \Psi-\gamma^{i}{ }_{j} D_{k} \Psi-\gamma_{j k} \mathcal{H} D^{i} B \\
& +D^{i} D_{j} D_{k} E .
\end{aligned}
$$

In analogous way one can compute the perturbation of the mixed components of the 
Ricci tensor as

$$
\begin{aligned}
a^{2} \delta_{\mathbf{X}_{\mathrm{S}}}^{(1)}\left[R_{0}^{0}\right]= & -6 \mathcal{H}^{\prime} \Phi-3 \mathcal{H}(\Phi+\Psi)-3 \Psi^{\prime \prime}+\nabla^{2}\left[-\Phi-\mathcal{H}\left(B-E^{\prime}\right)-B^{\prime}+E^{\prime}\right] \\
a^{2} \delta_{\mathbf{X}_{\mathrm{S}}^{(1)}\left[R_{i}^{0}\right]=} & 2 D_{i}\left(-\mathcal{H} \Phi-\Psi^{\prime}+K B\right) \\
a^{2} \delta_{\mathbf{X}_{\mathrm{S}}}^{(1)}\left[R_{0}^{i}\right]= & 2 D^{i}\left[\mathcal{H} \Phi+\Psi^{\prime}+\left(\mathcal{H}^{2}-\mathcal{H}^{\prime}\right) B\right] \\
a^{2} \delta_{\mathbf{X}_{\mathrm{S}}}^{(1)}\left[R_{j}^{i}\right]= & \gamma^{i}{ }_{j}\left\{-2 \Phi\left(2 \mathcal{H}^{2}+\mathcal{H}^{\prime}\right)+4 K \Psi-\mathcal{H}\left(\Phi^{\prime}+5 \Psi^{\prime}\right)\right. \\
& \left.\quad+\nabla^{2}\left[\Psi-\mathcal{H}\left(B-E^{\prime}\right)\right]\right\} \\
& +D^{i} D_{j}\left[-\Phi+\Psi-2 \mathcal{H}\left(B-E^{\prime}\right)-\left(B^{\prime}-E^{\prime \prime}\right)-4 K E\right]
\end{aligned}
$$

and of the Ricci curvature scalar

$$
\begin{aligned}
a^{2} \delta_{\mathbf{X}_{\mathrm{S}}}^{(1)}[R]= & -12\left(\mathcal{H}^{2}-\mathcal{H}^{\prime}\right) \Phi-6 \mathcal{H}\left(\Phi^{\prime}+3 \Psi^{\prime}\right)+12 K \Psi-6 \Psi^{\prime \prime} \\
& +\nabla^{2}\left[2(-\Phi+2 \Psi)-3 \mathcal{H}\left(2 B-E^{\prime}\right)-2\left(B^{\prime}-E^{\prime \prime}\right)-4 K E\right]
\end{aligned}
$$

With this results the mixed components of the first order perturbation for the Einstein tensor are given by

$$
\begin{aligned}
& a^{2} \delta_{\mathbf{X}_{\mathrm{S}}}^{(1)}\left[G_{0}^{0}\right]=6 \mathcal{H}^{2} \Phi-6 K \Psi+6 \mathcal{H} \Psi^{\prime} \\
& +\nabla^{2}\left[-2 \Psi+2 \mathcal{H}\left(B-E^{\prime}\right)+2 K E\right], \\
& a^{2} \delta_{\mathbf{X}_{\mathrm{S}}}^{(1)}\left[G^{0}{ }_{i}\right]=2 D_{i}\left(-\mathcal{H} \Phi-\Psi^{\prime}+K B\right) \text {, } \\
& a^{2} \delta_{\mathbf{X}_{\mathrm{S}}}^{(1)}\left[G_{0}^{i}\right]=2 D^{i}\left[\mathcal{H} \Phi+\Psi^{\prime}+\left(\mathcal{H}^{2}-\mathcal{H}^{\prime}\right) B\right], \\
& a^{2} \delta_{\mathbf{X}_{\mathrm{S}}}^{(1)}\left[G^{i}{ }_{j}\right]=\frac{1}{3} \gamma^{i}{ }_{j} \tilde{G}_{\mathbf{X}_{\mathrm{S}}}^{(1)}+\Delta^{i}{ }_{j}\left[-(\Phi-\Psi)-2 \mathcal{H}\left(B-E^{\prime}\right)\right. \\
& \left.-\left(B^{\prime}-E^{\prime \prime}\right)-2 K E\right] \text {; }
\end{aligned}
$$

where the trace-free part had been separated from the trace-full part given by:

$$
\begin{aligned}
\tilde{G}_{\mathbf{X}_{\mathrm{S}}}^{(1)}= & 6\left(\mathcal{H}^{2}+2 \mathcal{H}^{\prime}\right) \Phi+6 \mathcal{H}\left(\Phi^{\prime}+2 \Psi^{\prime}\right)-6 K \Psi+6 \Psi^{\prime \prime} \\
& +2 \nabla^{2}\left[(\Phi-\Psi)+2 \mathcal{H}\left(B-E^{\prime}\right)+\left(B^{\prime}-E^{\prime \prime}\right)\right] .
\end{aligned}
$$

\section{Gauge invariant variables}

The gauge transformation law for first order perturbations of a tensor, equation (A.20a), can be used to determine the transformation properties of the scalar fields $\mathbf{x}_{\mathrm{S}} \Phi^{(1)}, \mathbf{x}_{\mathrm{S}} \Psi^{(1)}, \mathbf{x}_{\mathrm{S}} B^{(1)}, \mathbf{x}_{\mathrm{S}} E^{(1)}$, defining the metric perturbations. Consider a second gauge field $\mathbf{Y}_{\mathrm{S}}$ parametrized as (A.28) but with scalar fields $\mathbf{Y}_{\mathrm{S}} \Phi^{(1)}, \mathbf{Y}_{\mathrm{S}} \Psi^{(1)}, \mathbf{Y}_{\mathrm{S}} B^{(1)}, \mathbf{Y}_{\mathrm{S}} E^{(1)}$ and consider the vector field generator of the Knight diffeomorphism of the gauge transformation $\mathbf{X}_{\mathrm{S}} \rightarrow \mathbf{Y}_{\mathrm{S}}$ given by

$$
\mathbf{V}_{(1)}=\mathbf{Y}_{\mathrm{S}}-\mathbf{X}_{\mathrm{S}}=T^{(1)} \partial_{0}+\left(D^{i} L^{(1)}+L^{(1) i}\right) \partial_{i} ; \quad D^{i} L_{i}^{(1)}=0
$$

according to the scalar-vector-tensor decomposition (A.22). 
Thus, by computing the Lie derivative of $\bar{g}$ along $\mathbf{V}_{1} \mathcal{L}_{\mathbf{V}_{(1)}} \bar{g}$ and separating the scalar modes for each component one can find

$$
\begin{aligned}
\mathbf{Y}_{\mathrm{S}} \Phi^{(1)}-\mathbf{x}_{\mathrm{S}} \Phi^{(1)} & =\mathcal{H} T^{(1)}+T^{(1)^{\prime}} \\
\mathbf{y}_{\mathrm{S}} \Psi^{(1)}-\mathbf{x}_{\mathrm{S}} \Psi^{(1)} & =-\mathcal{H} T^{(1)} \\
\mathbf{y}_{\mathrm{S}} B^{(1)}-\mathbf{x}_{\mathrm{S}} B^{(1)} & =L^{(1)^{\prime}}-T^{(1)} \\
\mathbf{y}_{\mathrm{S}} E^{(1)}-\mathbf{x}_{\mathrm{S}} E^{(1)} & =L^{(1)} .
\end{aligned}
$$

Therefore, it is not so difficult to see that the combinations

$$
\begin{aligned}
& \hat{\Phi}^{(1)}=\mathbf{x}_{\mathrm{S}} \Phi^{(1)}+\left(\mathbf{x}_{\mathrm{S}} B^{(1)}-\mathbf{x}_{\mathrm{S}} E^{(1) \prime}\right)^{\prime}+\mathcal{H}\left(\mathbf{x}_{\mathrm{S}} B^{(1)}-\mathbf{x}_{\mathrm{S}} E^{(1) \prime}\right) \\
& \hat{\Psi}^{(1)}=\mathbf{x}_{\mathrm{S}} \Psi^{(1)}-\mathcal{H}\left(\mathbf{x}_{\mathrm{S}} B^{(1)}-\mathbf{x}_{\mathrm{S}} E^{(1) \prime}\right)
\end{aligned}
$$

are gauge invariant scalar modes for the metric perturbations. However it is important to remark that there are other possible choses for this two variables, this is, another kind of combinations giving rise to gauge invariant quantities, see e.g. $\S 5.2$ in [2].

\section{A.3.4 Matter-energy perturbations}

The energy-momentum tensor at background level on a FLRW model is given by a perfect fluid one, see the discussion in section 1.1.

$$
\bar{T}_{\mu \nu}=(\bar{\rho}+\bar{p}) \bar{u}_{\mu} \bar{u}_{\nu}+\bar{p} \bar{g}_{\mu \nu},
$$

where $\bar{u}^{\mu}$ is the 4 -velocity of fundamental observers, $\bar{\rho}$ the energy density and $\bar{p}$ the pressure of the fluid. On a perturbed model, it can be generalized to have the form

$$
T_{\mu \nu}=(\rho+p) u_{\mu} u_{\nu}+p g_{\mu \nu}+\pi_{\mu \nu}
$$

where $\pi_{\mu \nu}$ accounts for anisotropic stress and therefore is a symmetric trace-free second rank tensor on the constant time hypersurfaces.

The up to first order four velocity is given by

$$
u^{\mu}=\frac{\mathrm{d} x^{\mu}}{\mathrm{d} t}=\frac{1}{a} \frac{\mathrm{d} x^{\mu}}{\mathrm{d} \eta}=\bar{u}^{\mu}+\delta u^{\mu},
$$

where $\bar{u}^{\mu}=a^{-1} \delta_{0}^{\mu}$ and should be a time-like four-vector, $g_{\mu \nu} u^{\mu} u^{\nu}=-1$. This normalization condition allows to derive the actual expressions for the components of the four velocity (covariant and contravariant) as

$$
\begin{aligned}
& u^{\mu}=\frac{1}{a}\left(1-\Phi, D^{i} v\right), \\
& u_{\mu}=a\left(-1-\Phi, D_{i} v+D_{i} B\right) .
\end{aligned}
$$

Therefore, the mixed components of the first order perturbations of the energy-momentum tensor should be found to be

$$
\begin{aligned}
\delta T^{0}{ }_{0} & =-\delta \rho, \\
\delta T^{0}{ }_{i} & =(\bar{\rho}+\bar{p})\left(D_{i} v+D_{i} B\right), \\
\delta T^{i}{ }_{j} & =\delta p \gamma^{i}{ }_{j}+a^{2} \delta \pi^{i}{ }_{j} .
\end{aligned}
$$


Here the anisotropic stress perturbation $\delta \pi_{i j}$ can be decomposed according to scalarvector-tensor decomposition (A.25) as

$$
\delta \pi^{i}{ }_{j}=\Delta^{i}{ }_{j} \Pi+D^{(i} \Pi_{j)}+\Pi_{j}^{i} ; \quad D^{i} \Pi_{i}=D^{i} \Pi_{i j}=\gamma_{i j} \Pi^{i j}=0,
$$

because the anisotropic stress is trace-free by construction.

Here the interest is on the scalar modes of first order perturbations. One can define a gauge fixing only scalar perturbations to the energy-momentum tensor by writting

$$
\begin{aligned}
\delta_{\mathbf{X}_{\mathrm{S}}}^{(1)}\left[T^{0}{ }_{0}\right] & =-\delta \rho, \\
\delta_{\mathbf{X}_{\mathrm{S}}}^{(1)}\left[T^{0}{ }_{i}\right] & =(\bar{\rho}+\bar{p})\left(D_{i} v+D_{i} B\right), \\
\delta_{\mathbf{X}_{\mathrm{S}}}^{(1)}\left[T^{i}{ }_{j}\right] & =\delta p \gamma^{i}{ }_{j}+a^{2} \Delta^{i}{ }_{j} \Pi .
\end{aligned}
$$

\section{Gauge invariant variables}

As for the metric perturbations, the gauge transformation law for first order perturbations of a tensor field, equation (A.20a), can be used to determine the transformation laws of the scalar modes of the components of the first order perturbation of the energy-momentum tensor, $\delta \rho, v, \delta p$ and $\Pi$. By computing the Lie derivative of the background energymomentum tensor along the vector field generator of the Knight diffeomorphism of gauge transformation $\mathbf{V}_{(1)}$ (equation $(\mathrm{A} .36)$ ) $\mathcal{L}_{\mathbf{V}_{(1)}} \bar{T}$ one can find

$$
\begin{aligned}
\mathbf{y}_{\mathrm{S}} \delta \rho-\mathbf{x}_{\mathrm{S}} \delta \rho & =\bar{\rho}^{\prime} T^{(1)} \\
\mathbf{Y}_{\mathrm{S}} v-\mathbf{x}_{\mathrm{S}} v & =-L^{(1) \prime}, \\
\mathbf{y}_{\mathrm{S}} \delta p-\mathbf{x}_{\mathrm{S}} \delta p & =\bar{p}^{\prime} T^{(1)}, \\
\mathbf{Y}_{\mathrm{S}} \Pi-\mathbf{x}_{\mathrm{S}} \Pi & =0 .
\end{aligned}
$$

Therefore, by also using the transformation laws for the metric scalar modes, equations (A.37), one can verify that the combinations

$$
\begin{aligned}
\hat{\delta \rho} & =\mathbf{x}_{\mathrm{S}} \delta \rho+\bar{\rho}^{\prime}\left(\mathbf{x}_{\mathrm{S}} v+\mathbf{x}_{\mathrm{S}} B^{(1)}\right), \\
\hat{\delta p} & =\mathbf{x}_{\mathrm{S}} \delta p+\bar{p}^{\prime}\left(\mathbf{x}_{\mathrm{S}} v+\mathbf{x}_{\mathrm{S}} B^{(1)}\right), \\
\hat{v} & =\mathbf{x}_{\mathrm{S}} v+\mathbf{x}_{\mathrm{S}} E^{(1) \prime}, \\
\hat{\Pi} & =\mathbf{x}_{\mathrm{S}} \Pi,
\end{aligned}
$$

are gauge invariant quantities. Again, these are just one possible set of combinations giving rise to gauge invariant quantities, there are other that we do not mention here because they are outside of the scope of the present work. See e.g. $§ 5.2$ in [2].

\section{A.3.5 First order Einstein field equations}

The first order Einstein field equations,

$$
\delta_{\mathbf{X}_{\mathrm{S}}}^{(1)}\left[G^{\mu}{ }_{\nu}\right]=\kappa \delta_{\mathbf{X}_{\mathrm{S}}}^{(1)}\left[T^{\mu}{ }_{\nu}\right]
$$

determine the evolution of the scalar modes of perturbations. Four independent scalar equations can be found from (A.48), given by the (00) and (i0) components and the trace 
and trace free part of $(i j)$ components. According to equations (A.35) and (A.43) one ends up with the system

$$
\begin{aligned}
\left(\nabla^{2}+3 K\right) \Psi-3 \mathcal{H}\left(\Psi^{\prime}+\mathcal{H} \Phi\right)+\mathcal{H} \nabla^{2}\left(E^{\prime}-B\right) & =a^{2} \frac{\kappa}{2} \delta \rho \\
\mathcal{H} \Phi+\Psi^{\prime}-K B & =-a^{2} \frac{\kappa}{2}(\bar{\rho}+\bar{p})(v+B)( \\
\Phi^{\prime \prime}+2 \mathcal{H} \Phi^{\prime}-K \Phi+\mathcal{H} \Psi^{\prime}+\left(2 \mathcal{H}^{\prime}+\mathcal{H}^{2}\right) \Psi & =a^{2} \frac{\kappa}{2} \delta p \\
\left(E^{\prime}-B\right)^{\prime}+2 \mathcal{H}\left(E^{\prime}-B\right)+(\Phi-\Psi) & =a^{2} \kappa \Pi
\end{aligned}
$$

\section{Total matter (pure dust) fluid}

For a pure dust-like energy-momentum tensor, pressure-less fluid, $p=\pi_{i j}=0$ so the system (A.49) can be reduced to

$$
\begin{aligned}
\left(\nabla^{2}+3 K\right) \Psi-3 \mathcal{H}\left(\Psi^{\prime}+\mathcal{H} \Phi\right)+\mathcal{H} \nabla^{2}\left(E^{\prime}-B\right) & =a^{2} \frac{\kappa}{2} \delta \rho, \\
\mathcal{H} \Phi+\Psi^{\prime}-K B & =-a^{2} \frac{\kappa}{2} \bar{\rho}(v+B), \\
\Phi^{\prime \prime}+2 \mathcal{H} \Phi^{\prime}-K \Phi+\mathcal{H} \Psi^{\prime}+\left(2 \mathcal{H}^{\prime}+\mathcal{H}^{2}\right) \Psi & =0 \\
\left(E^{\prime}-B\right)^{\prime}+2 \mathcal{H}\left(E^{\prime}-B\right)+(\Phi-\Psi) & =0 .
\end{aligned}
$$

This is a system of four equations for six degrees of freedom, four metric perturbations and two fluid quantities, the perturbation to the energy density $\delta \rho$ and the peculiar velocity potential $v$. However, taking into account the gauge freedom to choose this potentials, one knows from $\S$ A.3.3 and $\S$ A.3.4 that from the four metric degrees of freedom it can be constructed two gauge invariant quantities given by (A.38) and the two fluid ones can be combined to give the gauge invariant quantities (A.47).

Therefore, introducing the gauge invariant scalar modes of (A.38) and (A.47) and the gauge invariant fluctuation of matter, $\hat{\delta}:=\hat{\delta \rho} / \bar{\rho}$, the system (A.50) is equivalent to

$$
\begin{aligned}
\left(\nabla^{2}+3 K\right) \hat{\Phi}-3 \mathcal{H}\left(\hat{\Phi}^{\prime}+\mathcal{H} \hat{\Phi}\right) & =a^{2} \frac{\kappa}{2} \bar{\rho}(\hat{\delta}-3 \mathcal{H} \hat{v}), \\
\hat{\Phi}^{\prime}+\mathcal{H} \hat{\Phi} & =-a^{2} \frac{\kappa}{2} \bar{\rho} \hat{v} \\
\hat{\Phi}^{\prime \prime}+3 \mathcal{H} \hat{\Phi}^{\prime}+\left(2 \mathcal{H}^{\prime}+\mathcal{H}^{2}-K\right) \hat{\Phi} & =0 .
\end{aligned}
$$

This system of equations together with the background EFE, equations (1.11),

$$
\begin{aligned}
2 \mathcal{H}^{\prime}+\mathcal{H}^{2}+K & =-a^{2} \kappa \bar{p}=0, \\
\mathcal{H}^{2}+K & =a^{2} \frac{\kappa}{3} \bar{\rho}
\end{aligned}
$$

can be combined to eliminate terms with explicit dependence of conformal time derivatives of $\mathcal{H}$ and $\Phi$, this is, to obtain evolution equations for $\delta, v$ and $\Phi$,

$$
\begin{aligned}
\hat{\delta}^{\prime}+\left(\nabla^{2}+3 K\right) \hat{v} & =0 \\
\hat{v}^{\prime}+\mathcal{H} \hat{v}+\hat{\Phi} & =0 \\
\left(\nabla^{2}+3 K\right) \hat{\Phi} & =a^{2} \frac{\kappa}{2} \bar{\rho} \hat{\delta} .
\end{aligned}
$$


Since GR was assumed on top of the derivation of the system (A.53) the first two equations can be shown to be equivalent to the first order perturbations to the local conservation of energy-momentum tensor $\nabla_{\mu} T^{\mu}{ }_{\nu}=0$. That is because the EFE formally contain this equations according to the Bianchi identities.

The evolution equations (A.53) are equivalent in form to fluid equations obtained via the Poisson-Vlasov system in Newtonian theory. However, as long as they are a relativistic result according to the treatment presented, they have another interpretation. The system (A.53) accounts for evolution equations to gauge invariant, then physically observable degees of freedom, first order perturbations of matter fluid around a FLRW background model. 


\section{Appendix B}

\section{Scalar harmonic modes in the Universe}

In the present appendix we consider the problem of determine and characterize the scalar harmonic modes in FLRW Universes properly accounting for its spatial curvature. In order to do so, we closely follow the treatment of [35].

The goal is to construct the spherical harmonic decomposition of a scalar field defined on the constant time hypersurfaces of FLRW models. Such a decomposition represents a natural generalization of the standard Fourier decomposition used in the spatially flat analysis and is at the heart of the definition of two-point statistics for scalar fields in the Universe, see the chapter 2.

In order to construct an orthonormal complete set of harmonic functions one need to obtain a set of scalar functions satisfying the Helmholtz equation

$$
\left(\nabla^{2}+k^{2}\right) Q=0
$$

on the constant time hypersurfaces of an FLRW model, where the action of the Laplacian operator, $\nabla^{2} Q:=\gamma^{i j} D_{i} D_{j} Q^{1}$, can be expanded using the identity (see e.g. $§ 32.2 .4$ of [100])

$$
\nabla^{2} Q=\gamma^{i j}\left(\partial_{i} \partial_{j} Q-\Gamma_{i j}^{k} \partial_{k} Q\right)
$$

In spherical coordinates $x^{i}=(\chi, \theta, \varphi)$,

$$
\nabla^{2} Q=\left\{\begin{array}{l}
\frac{-K}{\sinh ^{2}(\chi)}\left[\frac{\partial}{\partial \chi}\left(\sinh ^{2}(\chi) \frac{\partial Q}{\partial \chi}\right)+\frac{1}{\sin (\theta)} \frac{\partial}{\partial \theta}\left(\sin (\theta) \frac{\partial Q}{\partial \theta}\right)+\frac{1}{\sin ^{2}(\theta)} \frac{\partial^{2} Q}{\partial \varphi^{2}}\right], \\
\frac{1}{\chi^{2}}\left[\frac{\partial}{\partial \chi}\left(\chi^{2} \frac{\partial Q}{\partial \chi}\right)+\frac{1}{\sin (\theta)} \frac{\partial}{\partial \theta}\left(\sin (\theta) \frac{\partial Q}{\partial \theta}\right)+\frac{1}{\sin ^{2}(\theta)} \frac{\partial^{2} Q}{\partial \varphi^{2}}\right], \\
\frac{K}{\sin ^{2}(\chi)}\left[\frac{\partial}{\partial \chi}\left(\sin ^{2}(\chi) \frac{\partial Q}{\partial \chi}\right)+\frac{1}{\sin (\theta)} \frac{\partial}{\partial \theta}\left(\sin (\theta) \frac{\partial Q}{\partial \theta}\right)+\frac{1}{\sin ^{2}(\theta)} \frac{\partial^{2} Q}{\partial \varphi^{2}}\right],
\end{array}\right.
$$

for $K<0,=0$ and $>0$ respectively.

Separating variables, $Q=X_{\ell}(\chi) Y_{\ell}^{m}(\theta, \varphi)$, it is not so difficult to see that the angular part solution is given by the Spherical Harmonic functions $Y_{\ell}^{m}(\theta, \varphi)$ and the radial part

\footnotetext{
${ }^{1}$ remember that $D_{i}$ denote the $i$ th component of the covariant derivative of a tensor in the constant time hypersurfaces.
} 
$X_{\ell}(\chi)$, associated with $Y_{\ell}^{m}$, should satisfy the radial equation

$$
\begin{array}{rl}
\frac{1}{\sinh ^{2}(\chi)} \frac{\partial}{\partial \chi}\left[\sinh ^{2}(\chi) \frac{\partial X_{\ell}}{\partial \chi}\right]+\left[\nu^{2}+1-\frac{\ell(\ell+1)}{\sinh ^{2}(\chi)}\right] X_{\ell}=0 & K<0 \\
\frac{1}{\chi^{2}} \frac{\partial}{\partial \chi}\left(\chi^{2} \frac{\partial X_{\ell}}{\partial \chi}\right)+\left[\nu^{2}-\frac{\ell(\ell+1)}{\chi^{2}}\right] X_{\ell}=0 & K=0 \\
\frac{1}{\sin ^{2}(\chi)} \frac{\partial}{\partial \chi}\left[\sin ^{2}(\chi) \frac{\partial X_{\ell}}{\partial \chi}\right]+\left[\nu^{2}-1-\frac{\ell(\ell+1)}{\sin ^{2}(\chi)}\right] X_{\ell}=0 & K>0
\end{array}
$$

where the adimensional eigenvalue $\nu$ was introduced by convenience via

$$
\begin{array}{lll}
k^{2}=-K\left(\nu^{2}+1\right) & \text { or } \quad \nu^{2}:=-\left(k^{2} / K+1\right), & K<0, \\
k^{2}=\nu^{2} & \text { or } \quad \nu^{2}:=k^{2}, & K=0, \\
k^{2}=K\left(\nu^{2}-1\right) & \text { or } \quad \nu^{2}:=\left(k^{2} / K-1\right), & K>0 .
\end{array}
$$

The solutions for this equations are known in the literature, see e.g. [101, 102] and the recent work in the context of Boltzmann codes [103], which we also follow closely in our treatment. For the $K=0$ case, the equation is nothing but the spherical Bessel equation,

$$
\frac{\mathrm{d}^{2} X_{\ell}}{\mathrm{d} \chi^{2}}+\frac{2}{\chi} \frac{\mathrm{d} X_{\ell}}{\mathrm{d} \chi}+\left[\nu^{2}-\frac{\ell(\ell+1)}{\chi^{2}}\right] X_{\ell}=0 ;
$$

while for the $K \neq 0$ cases, both equations can be reduced to the associated Legendre equation,

$$
\frac{\mathrm{d}^{2} Y_{\ell}}{\mathrm{d} \xi^{2}}+\frac{\cos (\xi)}{\sin (\xi)} \frac{\mathrm{d} Y_{\ell}}{\mathrm{d} \xi}+\left[\lambda(\lambda+1)-\frac{\mu^{2}}{\sin ^{2}(\xi)}\right] Y_{\ell}=0
$$

In Equation (B.4a) the changes of variables $\chi=: i \xi$ and $X_{\ell}(\chi)=: \sin ^{-1 / 2}(\xi) Y_{\ell}(\xi)$ should be introduced together with the identifications $\mu=\frac{1}{2}+\ell$ and $\lambda=-\frac{1}{2}+i \nu$; while in (B.4c) the corresponding changes are $\chi=: \xi$ and $X_{\ell}(\chi)=: \sin ^{-1 / 2}(\xi) Y_{\ell}(\xi)$ and the identifications $\mu=\frac{1}{2}+\ell$ and $\lambda=-\frac{1}{2}+\nu$.

The solutions for the $K=0$ case are spherical Bessel functions, two linearly independent solutions are then given by the first and second kind of this functions, $j_{\ell}(\chi)$ and $n_{\ell}(\chi)$; while for the $K \neq 0$ cases, solutions are given by associated Legendre functions $P_{\lambda}^{\mu}(\cos (\xi))$. Since for both cases ( $K$ negative or positive) $\mu$ is non-integer, one can choose $P_{\lambda}^{\mu}(\cos (\xi))$ as $P_{\lambda}^{-\mu}(\cos (\xi))$ as two linearly independent solutions. By imposing for the solutions to be regular at the origin, the part of the solutions proportional to $n_{\ell}$ and $P_{\lambda}^{-\nu}$ for the $K=0$ and $K \neq 0$ should necessarily vanish, so that one end up with conical or Mehler, spherical Bessel and toroidal or ring functions [104, 105] for negative, zero and positive curvature, respectively:

$$
\begin{aligned}
& X_{\ell}^{(-)}(\chi, \nu)=(-1)^{\ell} M_{\ell}^{(-)}(\nu) \sqrt{\frac{\pi}{2 \sinh (\chi)}} P_{-1 / 2+i \nu}^{-1 / 2-\ell}(\cosh (\chi)) \\
& X_{\ell}^{(0)}(\chi, \nu)=(-1)^{\ell} \nu^{\ell} j_{\ell}(\nu \chi) \\
& X_{\ell}^{(+)}(\chi, \nu)=(-1)^{\ell} M_{\ell}^{(+)}(\nu) \sqrt{\frac{\pi}{2 \sin (\chi)}} P_{-1 / 2+\nu}^{-1 / 2-\ell}(\cos (\chi)),
\end{aligned}
$$


where the normalization coefficients, given by

$$
M_{\ell}^{( \pm)}(\nu):= \begin{cases}1 & \ell=0 \\ \prod_{i=1}^{\ell}\left(\nu^{2} \pm i^{2}\right) & \ell \neq 0\end{cases}
$$

were chosen taking into account the recursion relations for the spherical Bessel and associated Legendre functions in order to keep simple its corresponding forms for the harmonic modes $X_{\ell}$. In fact, with these normalizations the derivatives and recursion relations acquire particularly symmetric forms:

$$
\begin{aligned}
& \frac{\partial}{\partial \chi}\left[\frac{X_{\ell}^{(-)}(\chi, \nu)}{\sinh ^{\ell}(\chi)}\right]=\frac{X_{\ell+1}^{(-)}(\chi, \nu)}{\sinh ^{\ell}(\chi)}, \\
& \frac{\partial}{\partial \chi}\left[\frac{X_{\ell}^{(0)}(\chi, \nu)}{\chi^{\ell}}\right]=\frac{X_{\ell+1}^{(0)}(\chi, \nu)}{\chi^{\ell}}, \\
& \frac{\partial}{\partial \chi}\left[\frac{X_{\ell}^{(+)}(\chi, \nu)}{\sin ^{\ell}(\chi)}\right]=\frac{X_{\ell+1}^{(+)}(\chi, \nu)}{\sin ^{\ell}(\chi)}
\end{aligned}
$$

and

$$
\begin{array}{r}
\left(\nu^{2}+\ell^{2}\right) X_{\ell-1}^{(-)}(\chi, \nu)+(2 \ell+1) \frac{\cosh (\chi)}{\sinh (\chi)} X_{\ell}^{(-)}(\chi, \nu)+X_{\ell+1}^{(-)}(\chi, \nu)=0 \\
\nu^{2} X_{\ell-1}^{(0)}(\chi, \nu)+\frac{2 \ell+1}{\chi} X_{\ell}^{(0)}(\chi, \nu)+X_{\ell+1}^{(0)}(\chi, \nu)=0 \\
\left(\nu^{2}-\ell^{2}\right) X_{\ell-1}^{(+)}(\chi, \nu)+(2 \ell+1) \frac{\cos (\chi)}{\sin (\chi)} X_{\ell}^{(+)}(\chi, \nu)+X_{\ell+1}^{(+)}(\chi, \nu)=0
\end{array}
$$

respectively [35, 102].

The $K>0$ case has an important special property. To cover the spacetime the radial coordinate $\chi$ must lie on the interval $[0, \pi]$ on the $K>0$ case, see 1.1. This introduces a boundary condition to be satisfied by the harmonic modes $X_{\ell}^{(+)}(\chi, \nu)$, the limits $\chi \rightarrow \pi$ and $\chi \rightarrow 0$ should coincide. The limit $\chi \rightarrow \pi$ can be thought as $x:=\cos (\chi) \rightarrow-1^{+}$and $\chi \rightarrow 0$ as $x \rightarrow 1^{-}$. Then, In order to relate the two limits one can use the connection formula (see e.g., §14.9 of [104])

$$
P_{\lambda}^{\mu}(-x)=\cos ((\lambda+\mu) \pi) P_{\lambda}^{\mu}(x)-\frac{2}{\pi} \sin ((\lambda+\mu) \pi) Q_{\lambda}^{\mu}(x),
$$

which establish the parity properties of Legendre functions. As was already mentioned, one can see that in the limit $x \rightarrow 1^{-}(\chi \rightarrow 0) P_{\lambda}^{\mu}(x)$ is regular while $Q_{\lambda}^{\mu}(x)$ diverges, therefore, for the boundary condition to be regular at $\chi=\pi$ the coefficient of $Q_{\lambda}^{\mu}(x)$ in (B.12) should be identically zero for all possible values of $\mu$ and $\lambda$, i.e., introducing $\mu=-\frac{1}{2}-\ell$ and $\lambda=-\frac{1}{2}+\nu$ (as should be for $K>0$ case) one finds the following condition to hold:

$$
\sin ((\nu-\ell-1) \pi)=0 .
$$

So that one should conclude that the possible values for $\nu$ must be integer. This is, for closed FLRW models the Laplace operator has a discrete spectrum. 
The above property is unique of the $K>0$ case. For the open and flat cases, $K<0$ and $K=0$ respectively, the radial coordinate should lie on $[0, \infty)$ in order to cover the spacetime, so no boundary condition should be imposed.

The expression (B.12) together with the above result that $\nu$ should be integer allow to study the parity properties of $X_{\ell}^{(+)}$functions. Let $\chi \in[0, \pi]$, then

$$
\begin{aligned}
X_{\ell}^{(+)}(\pi-\chi, \nu) & =(-1)^{\ell} M_{\ell}^{(+)}(\nu) \sqrt{\frac{\pi}{2 \sin (\pi-\chi)}} P_{-\frac{1}{2}+\nu}^{-\frac{1}{2}-\ell}(-\cos (\chi)) \\
& =(-1)^{\ell} M_{\ell}^{(+)}(\nu) \sqrt{\frac{\pi}{2 \sin (\chi)}} \cos ((\nu-\ell-1) \pi) P_{-\frac{1}{2}+\nu}^{-\frac{1}{2}-\ell}(\cos (\chi)) \\
& =(-1)^{\nu-\ell-1} X_{\ell}^{(+)}(\chi, \nu) .
\end{aligned}
$$

Thus, $X_{\ell}(\chi, \nu)$ is symmetric/anti-symmetric around $\chi=\pi / 2$ for $(\nu-\ell-1)$ even/odd.

For $\nu$ a positive integer the $X_{\ell}^{(+)}$functions can be represented in terms of the Gegenbauer (or ultraspherical) polynomials as

$$
X_{\ell}^{(+)}(\chi, \nu)=\frac{(-2)^{\ell} \ell ! \sin (\chi)}{\nu} C_{\nu-\ell-1}^{\ell+1}(\cos (\chi)) .
$$

This relation can be probed by using the general relation between Legendre and Gegenbauer functions (see e.g., $§ 14.3$ of [104]),

$$
P_{\lambda}^{\mu}(x)=\frac{2^{\mu} \Gamma(1-2 \mu) \Gamma(\lambda+\mu+1)}{\Gamma(\lambda-\mu+1) \Gamma(1-\mu)\left(1-x^{2}\right)^{\mu / 2}} C_{\lambda+\mu}^{\frac{1}{2}-\mu}(x),
$$

noting that in the $K>0$ case $\lambda+\mu=\nu-\ell-1$, which is the order of Gegenbauer polynomial, is a natural number; together with the fact that the normalization factor $M_{\ell}^{(+)}(\nu)$ can be written in terms of factorials as

$$
M_{\ell}^{(+)}(\nu)=\left[\prod_{n=1}^{\ell}(\nu-n)\right]\left[\prod_{n=1}^{\ell}(\nu+n)\right]=\frac{(\nu-1) !}{(\nu-\ell-1) !} \frac{(\ell+\nu) !}{\nu !}=\frac{(\nu+\ell) !}{\nu(\nu-\ell-1) !} .
$$

Computing explicitly,

$$
\begin{aligned}
X_{\ell}^{(+)}(\chi, \nu)= & (-1)^{\ell} M_{\ell}^{(+)}(\nu) \sqrt{\frac{\pi}{2 \sin (\chi)}} P_{-\frac{1}{2}+\nu}^{-\frac{1}{2}-\ell}(\cos (\chi)) \\
= & (-1)^{\ell} \frac{(\nu+\ell) !}{\nu(\nu-\ell-1) !} \sqrt{\frac{\pi}{2 \sin (\chi)}} \\
& \quad \times \frac{2^{-\frac{1}{2}-\ell} \Gamma(2 \ell+2) \Gamma(\nu-\ell)}{\Gamma(\nu+\ell+1) \Gamma\left(\ell+\frac{3}{2}\right) \sin ^{-\frac{1}{2}-\ell}(\chi)} C_{\nu-\ell-1}^{\ell+1}(\cos (\chi)) \\
= & \frac{(-1)^{\ell}}{\nu} 2^{\ell} \sin ^{\ell}(\chi) \ell ! C_{\nu-\ell-1}^{\ell+1}(\cos (\chi)),
\end{aligned}
$$

where in the last line the Legendre duplication formula (see e.g. $§ 7.4 .2$ from [100]) was applied to write

$$
\Gamma(2(\ell+1))=\frac{2^{2 \ell+1}}{\sqrt{\pi}} \Gamma(\ell+1) \Gamma\left(\ell+\frac{3}{2}\right) .
$$


The recurrence relations for the derivatives, Equations (B.10), can be rewritten as

$$
\begin{aligned}
\frac{1}{\sinh (\chi)} \frac{\partial}{\partial \chi}\left[\frac{X_{\ell}^{(-)}(\chi, \nu)}{\sinh ^{\ell}(\chi)}\right] & =\frac{X_{\ell+1}^{(-)}(\chi, \nu)}{\sinh ^{\ell+1}(\chi)} \\
\frac{1}{\chi} \frac{\partial}{\partial \chi}\left[\frac{X_{\ell}^{(0)}(\chi, \nu)}{\chi^{\ell}}\right] & =\frac{X_{\ell+1}^{(0)}(\chi, \nu)}{\chi^{\ell+1}} \\
\frac{1}{\sin (\chi)} \frac{\partial}{\partial \chi}\left[\frac{X_{\ell}^{(+)}(\chi, \nu)}{\sin ^{\ell}(\chi)}\right] & =\frac{X_{\ell+1}^{(+)}(\chi, \nu)}{\sin ^{\ell+1}(\chi)}
\end{aligned}
$$

so that it follows that

$$
\begin{array}{r}
{\left[\frac{1}{\sinh (\chi)} \frac{\partial}{\partial \chi}\right]^{m}\left[\frac{X_{\ell}^{(-)}(\chi, \nu)}{\sinh ^{\ell}(\chi)}\right]=\frac{X_{\ell+m}^{(-)}(\chi, \nu)}{\sinh ^{\ell+m}(\chi)},} \\
{\left[\frac{1}{\chi} \frac{\partial}{\partial \chi}\right]^{m}\left[\frac{X_{\ell}^{(0)}(\chi, \nu)}{\chi^{\ell}}\right]=\frac{X_{\ell+m}^{(0)}(\chi, \nu)}{\chi^{\ell+m}},} \\
{\left[\frac{1}{\sin (\chi)} \frac{\partial}{\partial \chi}\right]^{m}\left[\frac{X_{\ell}^{(+)}(\chi, \nu)}{\sin ^{\ell}(\chi)}\right]=\frac{X_{\ell+m}^{(+)}(\chi, \nu)}{\sin ^{\ell+m}(\chi)} .}
\end{array}
$$

This relations are valid for all $m, \ell \in \mathbb{N}$ and $\chi, \nu$ on the specific domains for each case. In particular, for $\ell=0$, they express a relation between $X_{0}$ and $X_{m}$ for every $m \in \mathbb{N}$. This relations can be rewritten on the following convenient way:

$$
\begin{aligned}
& X_{\ell}^{(-)}(\chi, \nu)=\sinh ^{\ell}(\chi)\left(\frac{1}{\sinh (\chi)} \frac{\partial}{\partial \chi}\right)^{\ell} X_{0}^{(-)}(\chi, \nu), \\
& X_{\ell}^{(0)}(\chi, \nu)=\chi^{\ell}\left(\frac{1}{\chi} \frac{\partial}{\partial \chi}\right)^{\ell} X_{0}^{(0)}(\chi, \nu) \\
& X_{\ell}^{(+)}(\chi, \nu)=\sin ^{\ell}(\chi)\left(\frac{1}{\sin (\chi)} \frac{\partial}{\partial \chi}\right)^{\ell} X_{0}^{(+)}(\chi, \nu) .
\end{aligned}
$$

The relations between $X_{\ell}$ and $X_{0}$ given by Equations (B.22) can be used to properly construct all the harmonic modes $X_{\ell}$. By solving the $\ell=0$ case of Equations (B.4) can be easily found that

$$
\begin{aligned}
X_{0}^{(-)} & =\frac{\sin (\nu \chi)}{\nu \sinh (\chi)}, \\
X_{0}^{(0)} & =\frac{\sin (\nu \chi)}{\nu \chi}, \\
X_{0}^{(+)} & =\frac{\sin (\nu \chi)}{\nu \sin (\chi)} .
\end{aligned}
$$


Thus, the following closed form for the harmonic modes holds:

$$
\begin{array}{ll}
X_{\ell}^{(-)}(\chi, \nu)=\frac{\sinh ^{\ell}(\chi)}{\nu} \frac{\mathrm{d}^{\ell}}{\mathrm{d}(\cosh (\chi))^{\ell}}\left(\frac{\sin (\nu \chi)}{\sinh (\chi)}\right), & \text { for } \quad \nu^{2} \geq 0, K<0 \\
X_{\ell}^{(0)}(\chi, \nu)=\frac{\chi^{\ell}}{\nu}\left(\frac{1}{\chi} \frac{\partial}{\partial \chi}\right)^{\ell}\left(\frac{\sin (\nu \chi)}{\chi}\right), & \text { for } \quad \nu^{2} \geq 0, K=0 ; \\
X_{\ell}^{(+)}(\chi, \nu)=\frac{(-1)^{\ell} \sin ^{\ell}(\chi)}{\nu} \frac{\mathrm{d}^{\ell}}{\mathrm{d}(\cos (\chi))^{\ell}}\left(\frac{\sin (\nu \chi)}{\sin (\chi)}\right), & \text { for } \quad \nu=2,3,4 \ldots, K>0
\end{array}
$$

In the limit $\chi \rightarrow 0$ all the $X_{\ell}$ functions behave like $\chi^{\ell}$, irrespective of the sign of $K$; of course, with appropriate constants of normalization. In other words,

$$
\lim _{\chi \rightarrow 0} X_{\ell} \propto \chi^{\ell}
$$

It follows then that at $\chi=0$ the only non-zero harmonic mode is the one with $\ell=0$, i.e.,

$$
X_{\ell}(\chi=0, \nu)=\delta_{\ell 0}
$$

Orthogonality and completeness relations for the $X_{\ell}$ functions follows from the same relations for the spherical Bessel and Legendre functions. It is convenient to introduce a different normalization from that adopted on (B.8). Define new normalized harmonic modes

$$
\begin{aligned}
& \widehat{X}_{\ell}^{(-)}(\chi, \nu):=\frac{X^{(-)}(\chi, \nu)}{\sqrt{M^{(-)}(\nu)}}=(-1)^{\ell} \sqrt{\frac{\pi M_{\ell}^{(-)}(\nu)}{2 \sinh (\chi)}} P_{-\frac{1}{2}+i \nu}^{-\frac{1}{2}-\ell}(\cosh (\chi)), \\
& \widehat{X}_{\ell}^{(0)}(\chi, \nu):=\frac{X^{(0)}(\chi, \nu)}{\nu^{\ell}}=(-1)^{\ell} j_{\ell}(\nu \chi), \\
& \widehat{X}_{\ell}^{(+)}(\chi, \nu):=\frac{X^{(+)}(\chi, \nu)}{\sqrt{M^{(+)}(\nu)}}=(-1)^{\ell} \sqrt{\frac{\pi M_{\ell}^{(+)}(\nu)}{2 \sin (\chi)}} P_{-\frac{1}{2}+\nu}^{-\frac{1}{2}-\ell}(\cos (\chi)) ;
\end{aligned}
$$

then orthogonality and recurrence relations can be written as:

$$
\begin{aligned}
4 \pi \int \mathrm{d} \chi \sinh ^{2}(\chi) \widehat{X}_{\ell}^{(-)}(\chi, \nu) \widehat{X}_{\ell}^{(-)}\left(\chi, \nu^{\prime}\right) & =\frac{2 \pi^{2}}{\nu^{2}} \delta_{\mathrm{D}}\left(\nu-\nu^{\prime}\right), \\
4 \pi \int \mathrm{d} \chi \chi^{2} \widehat{X}_{\ell}^{(0)}(\chi, \nu) \widehat{X}_{\ell}^{(0)}\left(\chi, \nu^{\prime}\right) & =\frac{2 \pi^{2}}{\nu^{2}} \delta_{\mathrm{D}}\left(\nu-\nu^{\prime}\right), \\
4 \pi \int \mathrm{d} \chi \sin ^{2}(\chi) \widehat{X}_{\ell}^{(+)}(\chi, \nu) \widehat{X}_{\ell}^{(+)}\left(\chi, \nu^{\prime}\right) & =\frac{2 \pi^{2}}{\nu^{2}} \delta_{\nu \nu^{\prime}}
\end{aligned}
$$

and

$$
\begin{aligned}
\int \frac{\mathrm{d} \nu \nu^{2}}{2 \pi^{2}} \widehat{X}_{\ell}^{(-)}(\chi, \nu) \widehat{X}_{\ell}^{(-)}\left(\chi^{\prime}, \nu\right) & =\frac{\delta_{\mathrm{D}}\left(\chi-\chi^{\prime}\right)}{4 \pi \sinh ^{2}(\chi)}, \\
\int \frac{\mathrm{d} \nu \nu^{2}}{2 \pi^{2}} \widehat{X}_{\ell}^{(0)}(\chi, \nu) \widehat{X}_{\ell}^{(0)}\left(\chi^{\prime}, \nu\right) & =\frac{\delta_{\mathrm{D}}\left(\chi-\chi^{\prime}\right)}{4 \pi \chi^{2}}, \\
\sum_{\nu=2}^{\infty} \frac{\mathrm{d} \nu \nu^{2}}{2 \pi^{2}} \widehat{X}_{\ell}^{(+)}(\chi, \nu) \widehat{X}_{\ell}^{(+)}\left(\chi^{\prime}, \nu\right) & =\frac{\delta_{\mathrm{D}}\left(\chi-\chi^{\prime}\right)}{4 \pi \sin ^{2}(\chi)}
\end{aligned}
$$


respectively. Note that for the $K>0$ case the discreteness of the spectrum should be taken into account.

Since according to the normalization coefficients, equation (B.9), irrespective of the curvature sign of the curvature, $M_{\ell=0}(\nu)=1$ for all possible values of $\nu$, the property of the scalar harmonic modes $X_{\ell}$ to be non-null only for $\ell=0$ (the lowest mode), Equation (B.26), also holds on the new normalization convention of Equations (B.27),

$$
\widehat{X}_{\ell}(\chi=0, \nu)=\delta_{\ell 0}
$$
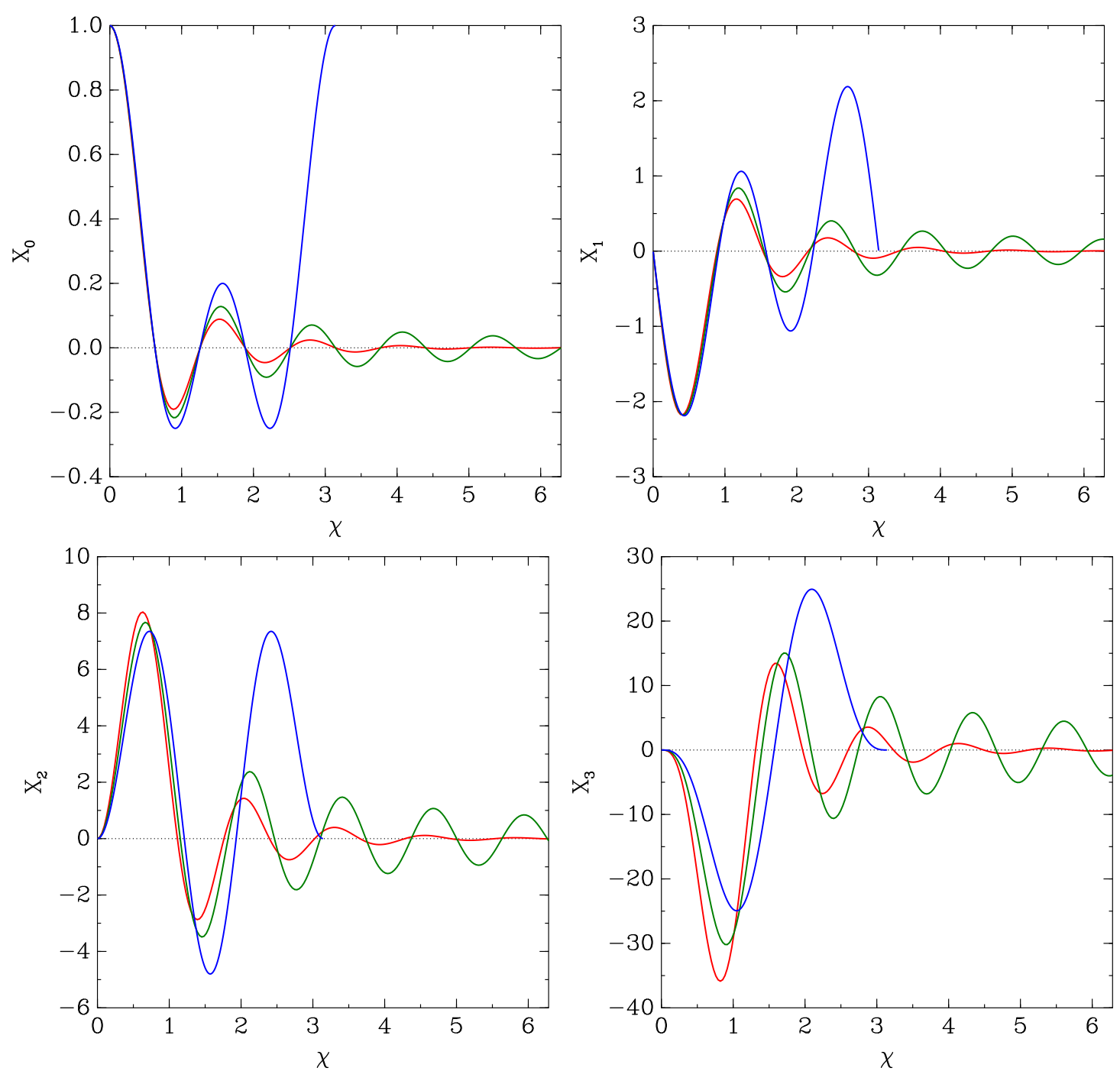

Figure B.1: Scalar harmonic modes $X_{\ell}, \ell=0,1,2,3$, for $\nu=5$, as a function of the radial coordinate $\chi$. The red, green and blue lines represent $K<0, K=0$ and $K>0$ cases respectively.

In order to illustrate some of the properties studied, from Equations (B.24) the $\ell=$ 
1,2,3 modes are constructed to be (the $\ell=0$ are given by Equations (B.23)):

$$
\begin{aligned}
& X_{1}^{(-)}= \frac{1}{\nu \sinh ^{2} \chi}[-\cosh (\chi) \sin (\nu \chi)+\nu \sinh (\chi) \cos (\nu \chi)] \\
& X_{2}^{(-)}= \frac{1}{\nu \sinh ^{3} \chi}\left\{\left[3-\left(\nu^{2}-2\right) \sinh ^{2}(\chi)\right] \sin (\nu \chi)-3 \nu \sinh (\chi) \cosh (\chi) \cos (\nu \chi)\right\} \\
& X_{3}^{(-)}=\frac{1}{\nu \sinh ^{4}(\chi)}\left\{\cosh (\chi)\left[-15+6\left(\nu^{2}-1\right) \sinh ^{2}(\chi)\right] \sin (\nu \chi)\right. \\
&\left.\quad+\nu \sinh (\chi)\left[15-\left(\nu^{2}-11\right) \sinh ^{2}(\chi)\right] \cos (\nu \chi)\right\}
\end{aligned}
$$

for $K<0$;

$$
\begin{aligned}
& X_{1}^{(0)}=\frac{1}{\nu \chi^{2}}[-\sin (\nu \chi)+\nu \chi \cos (\nu \chi)] \\
& X_{2}^{(0)}=\frac{1}{\nu \chi^{3}}\left[\left(3-\nu^{2} \chi^{2}\right) \sin (\nu \chi)-3 \nu \chi \cos (\nu \chi)\right], \\
& X_{3}^{(0)}=\frac{1}{\nu \chi^{4}}\left[\left(-15+6 \nu^{2} \chi^{2}\right) \sin (\nu \chi)+\nu \chi\left(15-\nu^{2} \chi^{2}\right) \cos (\nu \chi)\right],
\end{aligned}
$$

for $K=0$; and

$$
\begin{aligned}
& X_{1}^{(+)}=\frac{1}{\nu \sin ^{2}(\chi)}[-\cos (\chi) \sin (\nu \chi)+\nu \sin (\chi) \cos (\nu \chi)], \\
& X_{2}^{(+)}=\frac{1}{\nu \sin ^{3}(\chi)}\left\{\left[3-\left(\nu^{2}+2\right) \sin ^{2}(\chi)\right] \sin (\nu \chi)-3 \nu \sin (\chi) \cos (\chi) \cos (\nu \chi)\right\}, \\
& X_{3}^{(+)}=\frac{1}{\nu \sin ^{4}(\chi)}\left\{\cos (\chi)\left[-15+6\left(\nu^{2}+1\right) \sin ^{2}(\chi)\right] \sin (\nu \chi)\right. \\
&\left.\quad+\nu \sin (\chi)\left[15-\left(\nu^{2}+11\right) \sin ^{2}(\chi)\right] \cos (\nu \chi)\right\}
\end{aligned}
$$

for $K>0$.

Figure B.1 shows the radial harmonic modes $\ell=0,1,2,3$, for $\nu=5$, as a function of the radial coordinate $\chi, X_{\ell}$. The red, green and blue lines represent $K<0, K=0$ and $K>0$ cases respectively. It can be seen how the three different curvature cases for the different $\ell$ 's converge to be consistent with the flat case Universe model as $\chi \rightarrow 0$. The special feature of the closed Universe introduced by the boundary conditions at $\chi=0$ and $\chi=\pi$ are shown, together with its special property of being symmetric around $\chi=\pi / 2$ for $\nu-\ell-1$ even, so in the cases shown $(\nu=5)$, for $\ell$ even. Also, it can be seen that the $\ell=0$ multipoles are the only ones to go to the unity when $\chi \rightarrow 0$ and all the others converge to zero.

\section{B.1 Harmonic decomposition of a scalar field}

As a direct consequence of the completeness and orthogonal relations for the Harmonic Modes $\widehat{X}_{\ell}$ follows the proper generalization for curved spaces of the harmonic expansion for a general scalar field defined on the constant time hypersurfaces of FLRW models. Let $f=f(\chi, \theta, \varphi)$ be a scalar field on the constant time hypersurfaces of a FLRW model 
with spatial constant curvature $K$. The field $f$ can be expanded on the harmonic modes $X_{\ell}$ depending on the sign of $K$ as:

$$
\begin{aligned}
& \delta(\chi, \theta, \varphi)=\sum_{\ell=0}^{\infty} \sum_{m=-\ell}^{\ell} \int \frac{\mathrm{d} \nu \nu^{2}}{2 \pi^{2}} \delta_{\ell m}(\nu) \widehat{X}_{\ell}^{(-)}(\chi, \nu) Y_{\ell}^{m}(\theta, \varphi), \quad \text { for } K<0 ; \\
& \delta(\chi, \theta, \varphi)=\sum_{\ell=0}^{\infty} \sum_{m=-\ell}^{\ell} \int \frac{\mathrm{d} \nu \nu^{2}}{2 \pi^{2}} \delta_{\ell m}(\nu) \widehat{X}_{\ell}^{(0)}(\chi, \nu) Y_{\ell}^{m}(\theta, \varphi), \quad \text { for } K=0 \text {; } \\
& \delta(\chi, \theta, \varphi)=\sum_{\ell=0}^{\infty} \sum_{m=-\ell}^{\ell} \sum_{\nu=2}^{\infty} \frac{\nu^{2}}{2 \pi^{2}} \delta_{\ell m}(\nu) \widehat{X}_{\ell}^{(+)}(\chi, \nu) Y_{\ell}^{m}(\theta, \varphi), \quad \text { for } K>0 .
\end{aligned}
$$

The associated inverse expansions are given by:

$$
\begin{array}{ll}
\delta_{\ell m}(\nu)=4 \pi \int_{0}^{\infty} \mathrm{d} \chi \sinh ^{2}(\chi) \int \mathrm{d}^{2} \Omega \delta(\chi, \theta, \varphi) \widehat{X}_{\ell}^{(-)}(\chi, \nu) Y_{\ell}^{m *}(\theta, \varphi), & \text { for } K<0 \\
\delta_{\ell m}(\nu)=4 \pi \int_{0}^{\infty} \mathrm{d} \chi \chi^{2} \int \mathrm{d}^{2} \Omega \delta(\chi, \theta, \varphi) \widehat{X}_{\ell}^{(0)}(\chi, \nu) Y_{\ell}^{m *}(\theta, \varphi), & \text { for } K=0 \\
\delta_{\ell m}(\nu)=4 \pi \int_{0}^{\pi} \mathrm{d} \chi \sin ^{2}(\chi) \int \mathrm{d}^{2} \Omega \delta(\chi, \theta, \varphi) \widehat{X}_{\ell}^{(+)}(\chi, \nu) Y_{\ell}^{m *}(\theta, \varphi), & \text { for } K>0
\end{array}
$$

where the ${ }^{*}$ notation denotes complex conjugate. 


\section{Appendix C}

\section{Linear redshift space distortions in the Universe}

As discussed on $\S \mathrm{A} .3$ the evolution of the matter density fluctuations during structure formation in the Universe sources coherent motions in the matter described by the linear perturbations to the Einstein field equations (A.53). These peculiar velocities introduce a radial anisotropic distortion in redshift-space via a Doppler effect. This collective effect for all matter is known as redshift-space distortion (RSD) and provide a handle on the peculiar velocity field.

For a review of RSD see e.g. [106]. In general terms, the overall picture of RSD can be summarized as follows: in the linear regime (i.e., on sufficiently large scales), the distortion is a "squashing" in the radial (line of sight) direction, while in the nonlinear regime there is a stretching ("finger of god") effect. On large scales, the peculiar velocity of an infalling shell is small compared to its radius, causing the shell to appear squashed. On smaller scales, not only is the radius of a shell smaller, but also its peculiar infall velocity tends to be larger. For the shell that is just at turnaround, its peculiar velocity cancels the Hubble expansion, and it appears collapsed to a single velocity in redshift space. On even smaller scales, shells that are collapsing in proper coordinates appear inside out in redshift space. The combination of collapsing shells with previously collapsed, virialized shells gives rise to the finger of god shape.

The goal of the present appendix is to provide analytic results for the configurationspace representations of the redshift-space distortion operator, which determines the relation between real- and redshift-space matter fluctuations in the linear regime in the context of FLRW Universe models. In order to do so we closely follow the treatment of [35]. The Appendix begins with a brief discussion of redshift effect in the context of GR, then redshift-space radial distance $r_{(\mathrm{rs})}$ and (adimensional) coordinate $\chi_{(\mathrm{rs})}$ are introduced as the actual radial coordinates accessible with observations of the redshift of objects on the past ligh cone of an observer in FLRW models. The redshift-space matter fluctuation field $\delta^{(\mathrm{rs})}$ is then studied up to first order in cosmological perturbation theory and its relation with its real-space analog $\delta$ is used to define the redshift-space distortion operator $\widehat{R}$ and, finally introducing the biasing effect of tracers, the operator for galaxies $\widehat{R}_{\mathrm{g}}$ is constructed. 


\section{C.1 Redshift}

The redshift $z$ of a light emitting source as measured by an observer is defined in terms of the wavelength $\lambda$ of light by

$$
z:=\frac{\lambda_{\mathrm{obs}}-\lambda_{\mathrm{em}}}{\lambda_{\mathrm{em}}}=\frac{\Delta \lambda}{\lambda_{\mathrm{em}}}
$$

where the subscripts "obs" and "em" refers to observer and emitter, respectively. On astrophysical observations the measurement of redshifts is done by identifying absorption or emission lines for particular elements in spectra of distant objects, measuring their observed wavelength, and comparing this with with the known (laboratory) wavelength of the same lines for a source at rest. The interpretation depends on the assumption these spectra were the same in the past, i.e., that atomic physics does not change over cosmological scales.

The connection with GR is done via the optical limit of electrodynamics theory. The rate of change of any signal $g(\psi)$, described as an arbitrary function of the phase of radiation $\psi$ (see the discussion in $\S \mathrm{D} .1$ ), and measured by an observer moving with fourvelocity $u^{\mu}=\mathrm{d} x^{\mu} / \mathrm{d} \tau$ is given by

$$
\frac{\mathrm{d} g}{\mathrm{~d} \tau}=g^{\prime} k_{\mu} u^{\mu} \propto \nu
$$

where $\tau$ is the proper time of the observer and $\nu$ the frequency of the photon being propagated, $k^{\mu}$ is the propagation vector of radiation and $u^{\mu}$ is the four-velocity of the emitter/observer.

If two observers (emitter/observer) measure the rate of change of the same signal $g(\psi)$, the ratio of the respective measurements is given by

$$
1+z=\frac{\lambda_{\mathrm{obs}}}{\lambda_{\mathrm{em}}}=\frac{\nu_{\mathrm{em}}}{\nu_{\mathrm{obs}}}=\frac{\left.\left(u_{\mu} k^{\mu}\right)\right|_{\mathrm{em}}}{\left.\left(u_{\sigma} k^{\sigma}\right)\right|_{\mathrm{obs}}} .
$$

This relation is valid irrespective of the separation between emitter and observer and holds independent of any interpretation of redshift as Doppler or Gravitational.

\section{C.2 Redshift-space radial distance and coordinate}

The actual observed redshift of a source on FLRW models can be computed using first order perturbation theory. A first order redshift perturbation $\delta z$ can be written according to

$$
1+z_{\mathrm{obs}}=(1+\bar{z})(1+\delta z)=\frac{\nu_{\mathrm{s}}}{\nu_{\mathrm{obs}}}
$$

where the background redshift is given by $\bar{z}=a_{\text {obs }} / a_{\mathrm{s}}$. Here (and from now on) the subindex " $\mathrm{s}$ " is used to refer the source, as a synonym of emitter.

In consistency with GR, expanding the equation (C.3) up to first order, one should recognize three main contributions for the observed and source (emitter) rate of change of the signal, equation (C.2): the metric perturbations $\delta^{(1)}\left[g_{\mu \nu}\right] \bar{k}^{\mu} \bar{u}^{\nu}$, matter velocity perturbations $\bar{g}_{\mu \nu} \bar{k}^{\mu} \delta^{(1)}\left[u^{\nu}\right]$, and light trajectory perturbations $\bar{g}_{\mu \nu} \delta^{(1)}\left[k^{\mu}\right] \bar{u}^{\nu}$. During the present treatment of redshift space distortions the latter will be neglected, i.e., it will be assumed that light rays moves on unperturbed null-geodesics, $\delta^{(1)}\left[k^{\mu}\right]=0$. In other 
words, the comoving angular coordinates are assumed to be common in redshift and real space, only the radial coordinate and then distances are distorted in redshift space. Note that this assumption corresponds to a non fully relativistic treatment, because in a general treatment on cosmological perturbation theory up to first order one should take into account effects as convergence of light rays by lensing and the Integrated Sachs-Wolfe effect, both with physical origin on the full perturbed solutions to photon trajectories [2]. This approximation is made also to be consistent with present day analysis and literature on RSD.

Writing the perturbations in terms of the gauge-invariant scalar gravitational potential $\Phi$, peculiar velocity $v$ and the background light ray direction $\mathbf{n}$ (line of sight to the source) one finds

$$
\delta z=W_{\mathrm{s}}-W_{0}+\left(\left.\Phi\right|_{0}-\left.\Phi\right|_{\mathrm{s}}\right)
$$

where

$$
W_{\mathrm{s}}=\gamma_{i j} n^{i} D^{j} v \quad \text { and } \quad W_{0}=\gamma_{i j} n^{i} D^{j} v_{0}
$$

are the line of sight components of the peculiar velocities of source and observer, respectively. Its difference on (C.5) represent a Doppler term contribution. $W_{\mathrm{s}}$ is a quantity to be determined by observations on the light cone, unlike $W_{0}$ which is related with the reference system in which the measurements are done. $W_{0}$ can be estimated from CMB anisotropies measurements, since in the standard interpretation of CMB observations the harmonic dipole of temperature-temperature correlation function on the sky is directly related with this quantity $[4,2]$.

The term in brackets on the redshift perturbation (C.5) represents a Sachs-Wolfe contribution from the difference of the gravitational potential at observer and source. It will be neglected on the present discussion. Under this assumptions one should recover the standard results in the literature coming from analysis of pure Doppler contribution [33].

For convenience, one introduce the redshift-space comoving distance as

$$
s(z):=r\left(z_{\mathrm{obs}}\right)=\int_{0}^{z+(1+z)\left(W-W_{0}\right)} \frac{\mathrm{d} z^{\prime}}{H\left(z^{\prime}\right)}
$$

physically it represents the apparent comoving distance of an object in redshift space that is originally at redshift $z$ in real space and is shifted by its own peculiar velocity.

From the redshift-space comoving distance a redshift-space analog of the comoving radial coordinate $\chi$ can be constructed as

$$
\chi_{(\mathrm{rs})}=\chi\left(z_{\mathrm{obs}}\right)= \begin{cases}|K|^{1 / 2} s(z) & K \neq 0 \\ s(z) & K=0,\end{cases}
$$

defining the (adimensional) redshift-space radial coordinate.

Then, one can obtain the up to first order perturbative expansion of the redshift-space radial comoving distance $\left(W_{\mathrm{s}, 0}\right.$ are in fact perturbations of first order),

$$
s(z)=\int_{0}^{z} \frac{\mathrm{d} z^{\prime}}{H\left(z^{\prime}\right)}+\int_{z}^{z+(1+z)\left(W-W_{0}\right)} \frac{\mathrm{d} z^{\prime}}{H\left(z^{\prime}\right)}=r(z)+\frac{1+z}{H(z)}\left(W-W_{0}\right),
$$

and its adimensional equivalent, the redshift-space radial comoving coordinate,

$$
\chi_{(\mathrm{rs})}(z)=\chi(z)+\frac{1+z}{H(z)}\left(U-U_{0}\right)
$$


where

$$
U:= \begin{cases}|K|^{1 / 2} W & K \neq 0 \\ W & K=0\end{cases}
$$

\section{C.3 Redshift-space distorted fluctuations of matter}

Here we construct an up to first order perturbation of the fluctuation of the number density of objects in redshift space. Begin by assuming the number density of objects in a specific observation and in real space $n$ is given by the underlying matter density $\rho(\chi, \theta, \varphi)$ multiplied by a selection function $\Phi$, i.e.

$$
n(\chi, \theta, \varphi)=\Phi(\chi, \theta, \varphi) \rho(\chi, \theta, \varphi) .
$$

Therefore, for the real-space fluctuations of the number density of objects one have

$$
\delta=\frac{n}{\bar{\rho} \Phi}-1 .
$$

This relation inspire the definition of the redshift-space number of objects fluctuation,

$$
\delta^{(\mathrm{rs})}(\chi, \theta, \varphi):=\frac{n^{(\mathrm{rs})}\left(\chi_{(\mathrm{rs})}, \theta, \varphi\right)}{\bar{\rho} \Phi\left(\chi_{(\mathrm{rs})}, \theta, \varphi\right)}-1,
$$

that is, the number of object fluctuation evaluated on the redshift-space radial coordinate $\chi(\mathrm{rs})$.

The number density of observed objects in real-space $n(\chi, \theta, \varphi)$ and its redshift-space analogue $n^{(\mathrm{rs})}\left(\chi_{(\mathrm{rs})}, \theta, \varphi\right)$ should be related according to a number conservation by

$$
\begin{array}{rlrl}
n^{(\mathrm{rs})}\left(\chi_{(\mathrm{rs})}, \theta, \varphi\right) \sinh ^{2}\left(\chi_{(\mathrm{rs})}\right) \mathrm{d} \chi_{(\mathrm{rs})} \mathrm{d}^{2} \Omega & =n(\chi, \theta, \varphi) \sinh ^{2}(\chi) \mathrm{d} \chi \mathrm{d}^{2} \Omega & \text { for } \quad K<0, \\
n^{(\mathrm{rs})}\left(\chi_{(\mathrm{rs})}, \theta, \varphi\right) \chi_{(\mathrm{rs})}^{2} \mathrm{~d} \chi_{(\mathrm{rs})} \mathrm{d}^{2} \Omega=n(\chi, \theta, \varphi) \chi^{2} \mathrm{~d} \chi \mathrm{d}^{2} \Omega & \text { for } \quad K=0, \\
n^{(\mathrm{rs})}\left(\chi_{(\mathrm{rs})}, \theta, \varphi\right) \sin ^{2}\left(\chi_{(\mathrm{rs})}\right) \mathrm{d} \chi_{(\mathrm{rs})} \mathrm{d}^{2} \Omega=n(\chi, \theta, \varphi) \sin ^{2}(\chi) \mathrm{d} \chi \mathrm{d}^{2} \Omega & \text { for } \quad K<0,
\end{array}
$$

where $n$ is evaluated on the light cone. Therefore one may conclude that

$$
n^{(\mathrm{rs})}\left(\chi_{(\mathrm{rs})}, \theta, \varphi\right)=n(\chi, \theta, \varphi) \times \begin{cases}{\left[\frac{\sinh ^{2}\left(\chi_{(\mathrm{rs})}\right)}{\sinh ^{2}(\chi)}\left(\frac{\partial \chi_{(\mathrm{rs})}}{\partial \chi}\right)\right]^{-1}} & K<0 \\ {\left[\frac{\chi_{(\mathrm{rs})}^{2}}{\chi^{2}}\left(\frac{\partial \chi_{(\mathrm{rs})}}{\partial \chi}\right)\right]^{-1}} & K=0 \\ {\left[\frac{\sin ^{2}\left(\chi_{(\mathrm{rs})}\right)}{\sin ^{2}(\chi)}\left(\frac{\partial \chi_{(\mathrm{rs})}}{\partial \chi}\right)\right]^{-1}} & K>0 .\end{cases}
$$

Now proceed to expand up to first order the expression for the fluctuation in redshiftspace, (C.14). In order to perform a perturbative expansion of the selection function term in (C.14) one can formally perform a taylor expansion around $\chi$ of the right hand side, considered as a function of $\chi_{(\mathrm{rs})}$; that's because the difference $\chi_{(\mathrm{rs})}-\chi$ is a perturbation of first order according to (C.10). The expansion of $\Phi$ can then be written as

$$
\begin{aligned}
\Phi\left(\chi_{(\mathrm{rs})}, \theta, \varphi\right) & =\Phi(\chi, \theta, \varphi)+\frac{\partial \Phi(\chi, \theta, \varphi)}{\partial \chi}\left(\chi_{(\mathrm{rs})}-\chi\right) \\
& =\Phi(\chi, \theta, \varphi)\left\{1+\frac{\partial \ln \Phi(\chi, \theta, \varphi)}{\partial \chi}\left[\frac{1+z}{H(z)}\left(U-U_{0}\right)\right]\right\},
\end{aligned}
$$


so that we can write

$$
\frac{1}{\Phi\left(\chi_{(\mathrm{rs})}, \theta, \varphi\right)}=\frac{1}{\Phi(\chi, \theta, \varphi)}\left\{1-\frac{\partial \ln \Phi(\chi, \theta, \varphi)}{\partial \chi}\left[\frac{1+z}{H(z)}\left(U-U_{0}\right)\right]\right\}
$$

Now consider the perturbative expansion of the relation between the number densities in redshift- and real-space $n^{(\mathrm{rs})}$ and $n$, equation (C.15). The derivative term on the right-hand-side of (C.15) can be computed by nothing

$$
\frac{\partial \chi_{(\mathrm{rs})}}{\partial \chi}=1+\frac{\partial}{\partial \chi}\left[\frac{1+z}{H(z)}\left(U-U_{0}\right)\right],
$$

so that

$$
\left(\frac{\partial \chi_{(\mathrm{rs})}}{\partial \chi}\right)^{-1}=1-\frac{\partial}{\partial \chi}\left[\frac{1+z}{H(z)}\left(U-U_{0}\right)\right]
$$

The rest of the factor follows by expanding around $\chi$, as for the selection function above. Considering

$$
\begin{aligned}
\sinh ^{2}\left(\chi_{(\mathrm{rs})}\right) & =\sinh ^{2}(\chi)+2 \cosh (\chi) \sinh (\chi)\left[\chi_{(\mathrm{rs})}-\chi\right] \\
\chi_{(\mathrm{rs})}^{2} & =\chi^{2}+2 \chi\left[\chi_{(\mathrm{rs})}-\chi\right] \\
\sin ^{2}\left(\chi_{(\mathrm{rs})}\right) & =\sin ^{2}(\chi)+2 \cos (\chi) \sin (\chi)\left[\chi_{(\mathrm{rs})}-\chi\right]
\end{aligned}
$$

one has

$$
\begin{gathered}
{\left[\frac{\sinh ^{2}\left(\chi_{(\mathrm{rs})}\right)}{\sinh ^{2}(\chi)}\right]^{-1}=1-2 \frac{\cosh (\chi)}{\sinh (\chi)}\left[\chi_{(\mathrm{rs})}-\chi\right]=1-2 \frac{\cosh (\chi)}{\sinh (\chi)}\left[\frac{1+z}{H(z)}\left(U-U_{0}\right)\right]} \\
\left(\frac{\chi_{(\mathrm{rs})}^{2}}{\chi^{2}}\right)^{-1}=1-2 \frac{1}{\chi}\left[\chi_{(\mathrm{rs})}-\chi\right]=1-2 \frac{1}{\chi}\left[\frac{1+z}{H(z)}\left(U-U_{0}\right)\right] \\
{\left[\frac{\sin ^{2}\left(\chi_{(\mathrm{rs})}\right)}{\sin ^{2}(\chi)}\right]^{-1}=1-2 \frac{\cos (\chi)}{\sin (\chi)}\left[\chi_{(\mathrm{rs})}-\chi\right]=1-2 \frac{\cos (\chi)}{\sin (\chi)}\left[\frac{1+z}{H(z)}\left(U-U_{0}\right)\right]}
\end{gathered}
$$

Therefore, by combining equations (C.19) and (C.21) one has, up to first order,

$$
\begin{aligned}
{\left[\frac{\sinh ^{2}\left(\chi_{(\mathrm{rs})}\right)}{\sinh ^{2}(\chi)}\left(\frac{\partial \chi_{(\mathrm{rs})}}{\partial \chi}\right)\right]^{-1}=} & -2 \frac{\cosh (\chi)}{\sinh (\chi)}\left[\frac{1+z}{H(z)}\left(U-U_{0}\right)\right] \\
& -\frac{\partial}{\partial \chi}\left[\frac{1+z}{H(z)}\left(U-U_{0}\right)\right] \\
{\left[\frac{\chi_{(\mathrm{rs})}^{2}}{\chi^{2}}\left(\frac{\partial \chi_{(\mathrm{rs})}}{\partial \chi}\right)\right]^{-1}=} & 1-2 \frac{1}{\chi}\left[\frac{1+z}{H(z)}\left(U-U_{0}\right)\right]-\frac{\partial}{\partial \chi}\left[\frac{1+z}{H(z)}\left(U-U_{0}\right)\right] \\
{\left[\frac{\sin ^{2}\left(\chi_{(\mathrm{rs})}\right)}{\sin ^{2}(\chi)}\left(\frac{\partial \chi_{(\mathrm{rs})}}{\partial \chi}\right)\right]^{-1}=} & 1-2 \frac{\cos (\chi)}{\sin (\chi)}\left[\frac{1+z}{H(z)}\left(U-U_{0}\right)\right] \\
& -\frac{\partial}{\partial \chi}\left[\frac{1+z}{H(z)}\left(U-U_{0}\right)\right]
\end{aligned}
$$


Combining the results (C.17) and (C.22) one can arrive to

$$
\begin{aligned}
& \frac{1}{\Phi\left(\chi_{(\mathrm{rs})}, \theta, \varphi\right)}\left[\frac{\sinh ^{2}\left(\chi_{(\mathrm{rs})}\right)}{\sinh ^{2}(\chi)}\left(\frac{\partial \chi_{(\mathrm{rs})}}{\partial \chi}\right)\right]^{-1}=\frac{1}{\Phi(\chi, \theta, \varphi)}\left\{1-\frac{\cosh (\chi)}{\sinh (\chi)}\right. \\
& {\left[2+\frac{\partial \ln \Phi(\chi, \theta, \varphi)}{\partial \ln \sinh (\chi)}\right]\left[\frac{1+z}{H(z)}\left(U-U_{0}\right)\right]} \\
& \left.-\frac{\partial}{\partial \chi}\left[\frac{1+z}{H(z)}\left(U-U_{0}\right)\right]\right\} \\
& \frac{1}{\Phi\left(\chi_{(\mathrm{rs})}, \theta, \varphi\right)}\left[\frac{\chi_{(\mathrm{rs})}^{2}}{\chi^{2}}\left(\frac{\partial \chi_{(\mathrm{rs})}}{\partial \chi}\right)\right]^{-1}=\frac{1}{\Phi(\chi, \theta, \varphi)}\left\{1-\frac{1}{\chi}\right. \\
& {\left[2+\frac{\partial \ln \Phi(\chi, \theta, \varphi)}{\partial \ln \chi}\right]\left[\frac{1+z}{H(z)}\left(U-U_{0}\right)\right]} \\
& \left.-\frac{\partial}{\partial \chi}\left[\frac{1+z}{H(z)}\left(U-U_{0}\right)\right]\right\} \text {; } \\
& \frac{1}{\Phi\left(\chi_{(\mathrm{rs})}, \theta, \varphi\right)}\left[\frac{\sin ^{2}\left(\chi_{(\mathrm{rs})}\right)}{\sin ^{2}(\chi)}\left(\frac{\partial \chi_{(\mathrm{rs})}}{\partial \chi}\right)\right]^{-1}=\frac{1}{\Phi(\chi, \theta, \varphi)}\left\{1-\frac{\cos (\chi)}{\sin (\chi)}\right. \\
& {\left[2+\frac{\partial \ln \Phi(\chi, \theta, \varphi)}{\partial \ln \sin (\chi)}\right]\left[\frac{1+z}{H(z)}\left(U-U_{0}\right)\right]} \\
& \left.-\frac{\partial}{\partial \chi}\left[\frac{1+z}{H(z)}\left(U-U_{0}\right)\right]\right\} \text {. }
\end{aligned}
$$

In order to simplify this expressions it is convenient to write

$$
\frac{\partial}{\partial \chi}\left[\frac{1+z}{H(z)}\left(U-U_{0}\right)\right]=\frac{\partial}{\partial \chi}\left[\frac{1+z}{H(z)} U\right]-\frac{\partial}{\partial \chi}\left[\frac{1+z}{H(z)}\right] U_{0},
$$

where the fact that the peculiar velocity of the observer does not depend on the radial comoving coordinate was used (note that it clearly can not depend on the redshift) and to absorv the selction function dependent term into a function,

$$
A(\chi):= \begin{cases}\frac{\cosh (\chi)}{\sinh (\chi)}\left[2+\frac{\partial \ln \Phi(\chi, \theta, \varphi)}{\partial \ln \sinh (\chi)}\right] & K<0 \\ \frac{1}{\chi}\left[2+\frac{\partial \ln \Phi(\chi, \theta, \varphi)}{\partial \ln \chi}\right] & K=0 \\ \frac{\cos (\chi)}{\sin (\chi)}\left[2+\frac{\partial \ln \Phi(\chi, \theta, \varphi)}{\partial \ln \sin (\chi)}\right] & K=0 .\end{cases}
$$

By doing this one finally arrives to the following relation between redshift- and real-space linear matter fluctuations:

$$
\begin{aligned}
\delta^{(\mathrm{rs})}(\chi, \theta, \varphi)= & \delta(\chi, \theta, \varphi)-\frac{\partial}{\partial \chi}\left[\frac{1+z}{H(z)} U\right]-\frac{1+z}{H(z)} A(\chi) U \\
& +\left\{\frac{1+z}{H(z)} A(\chi)+\frac{\partial}{\partial \chi}\left[\frac{1+z}{H(z)}\right]\right\} U_{0}
\end{aligned}
$$




\section{C.4 Redshift-space distortion operator}

It is possible to formally introduce a redshift-space distortion operator $\widehat{R}$ as the operator that transforms the density contrast at present time in real space to that in redshift-space in the reference frame for which the observer velocity is null. From equation (C.26)

$$
\delta^{(\mathrm{rs})}(\chi, \theta, \varphi)=D(t) \widehat{R}\left[\delta_{0}(\chi, \theta, \varphi)\right]+\left\{\frac{1+z}{H(z)} A(\chi)+\frac{\partial}{\partial \chi}\left[\frac{1+z}{H(z)}\right]\right\} U_{0}
$$

where the action of the operator,

$$
\begin{aligned}
D(t) \widehat{R}\left[\delta_{0}(\chi, \theta, \varphi)\right] & =\delta(\chi, \theta, \varphi)-\frac{\partial}{\partial \chi}\left[\frac{1+z}{H(z)} U\right]-\frac{1+z}{H(z)} A(\chi) U \\
& =D(t)\left\{\delta_{0}(\chi, \theta, \varphi)-\frac{1}{D(t)}\left[\frac{\partial}{\partial \chi} \frac{1+z}{H(z)}+\frac{1+z}{H(z)} A(\chi)\right] U\right\},
\end{aligned}
$$

can be simplified to

$$
\widehat{R}\left[\delta_{0}(\chi, \theta, \varphi)\right]=\delta_{0}(\chi, \theta, \varphi)-f\left[\frac{\partial}{\partial \chi}+\alpha(\chi)\right]\left[\frac{U}{a H D f}\right]
$$

with $\alpha(\chi)$ a function encoding the selection effects,

$$
\alpha(\chi):=A(\chi)+\frac{\partial \ln (D f)}{\partial \chi}= \begin{cases}\frac{\cosh (\chi)}{\sinh (\chi)}\left[2+\frac{\partial \ln (D f \Phi)}{\partial \ln \sinh (\chi)}\right] & K<0, \\ \frac{1}{\chi}\left[2+\frac{\partial \ln (D f \Phi)}{\partial \ln \chi}\right] & K=0, \\ \frac{\cos (\chi)}{\sin (\chi)}\left[2+\frac{\partial \ln (D f \Phi)}{\partial \ln \sin (\chi)}\right] & K=0 .\end{cases}
$$

Note that equation (C.28) is nothing but a generalization of the original formula deduced by Kaiser in 1987 (equation (3.3) in [33]).

From the equation (C.28) it is clear that in order to find a closed expression for the $\widehat{R}$ operator it is necessary to find a relation between the line of sight derivative of the matter velocity field $U$ and the fluctuation of matter field today $\delta_{0}$. Such a relation appears naturally in linear cosmological perturbation theory where the peculiar velocity field has the solution

$$
v=-a H G f\left(\nabla^{2}+3 K\right)^{-1} \delta_{0}
$$

were $G$ is the linear growing factor and $f=\mathrm{d} \ln G / \mathrm{d} \ln a$, see section 1.2.1.

Therefore, the line of sight derivative for the velocity field $W$ can be written as

$$
W=\gamma_{i j} n^{i} D^{j} v=-a H D f \frac{\partial}{\partial r}\left(\nabla^{2}+3 K\right)^{-1} \delta_{0}
$$

where the photon trajectory from the source was assumed purely radial (without loss of generality) and spatial coordinates $x^{i}=(r, \theta, \varphi)$ was chosen. Thus, after changing the spatial coordinates to $x^{i}=(\chi, \theta, \varphi)$, one has

$$
U(\chi, \theta, \varphi)=-a H D f \begin{cases}|K| \partial_{\chi}\left(\nabla^{2}+3 K\right)^{-1} \delta_{0}(\chi, \theta, \varphi) & K \neq 0 \\ \partial_{\chi}\left(\nabla^{2}\right)^{-1} \delta_{0}(\chi, \theta, \varphi) & K=0\end{cases}
$$


Combining (C.32) and (C.28) one finally arrive to

$$
\widehat{R}=\widehat{1}+f(z) \begin{cases}|K|\left[\partial_{\chi}+\alpha(\chi)\right] \partial_{\chi}\left(\nabla^{2}+3 K\right)^{-1} & K \neq 0 \\ {\left[\partial_{\chi}+\alpha(\chi)\right] \partial_{\chi}\left(\nabla^{2}\right)^{-1}} & K=0 .\end{cases}
$$

The redshift-space distortion operator $\widehat{R}$ relates the fields $\delta^{(\mathrm{rs})}$ and $\delta_{0}$, which are associated to the total matter in the Universe, including barionic matter and cold dark matter. However, astrophysical observations do not allow to directly probe this fields of fluctuations. Observations can be done of luminous matter in the Universe, observed in some astrophysical objects such as galaxies or quasi stellar objects (QSO), a.k.a quasars. In order to relate the observed quantities for such objects with the fluctuation fields one assume such objects to be biased tracers of the total matter distribution in the Universe. that is, consider them as tracers e.g. galaxies, the galaxy fluctuation field in the Universe $\delta_{\mathrm{g}}$, whose properties can be inferred from astrophysical observations, is assumed to have a relation with the underlying total matter fluctuation of the form

$$
\delta_{\mathrm{g}}\left(\eta, x^{i}\right)=b_{\mathrm{g}}\left(z, x^{i}\right) \delta\left(\eta, x^{i}\right)=b_{\mathrm{g}}\left(z, x^{i}\right) G(z) \delta_{0}\left(x^{i}\right),
$$

specified by a bias function, $b_{\mathrm{g}}\left(z, x^{i}\right)$, which in principle should be a function of the redshift to the object on the observer's past lightcone and the object position, giving rise to different clustering scales and strengths for different tracers on different epochs and scales in the Universe. For simplicity, the bias function is usually assumed scale independent, that is independent of the position, $b_{\mathrm{g}}=b_{\mathrm{g}}(z)$, see the discussion on section 1.4.1.

By taking into account the bias of specific tracers it is possible to construct redshiftspace operator for such tracers according to (compare with equation (C.27))

$$
\delta_{\mathrm{g}}^{(\mathrm{rs})}(\chi, \theta, \varphi)=b_{\mathrm{g}}(z) D(z) \widehat{R}_{\mathrm{g}}\left[\delta_{0}(\chi, \theta, \varphi)\right]+\left\{\frac{1+z}{H(z)} A(\chi)+\frac{\partial}{\partial \chi}\left[\frac{1+z}{H(z)}\right]\right\} U_{0},
$$

from which one can see that (compare with equation (C.33))

$$
\widehat{R}_{\mathrm{g}}=\widehat{1}+\beta_{\mathrm{g}}(z) \begin{cases}|K|\left[\partial_{\chi}+\alpha(\chi)\right] \partial_{\chi}\left(\nabla^{2}+3 K\right)^{-1} & K \neq 0 \\ {\left[\partial_{\chi}+\alpha(\chi)\right] \partial_{\chi}\left(\nabla^{2}\right)^{-1}} & K=0\end{cases}
$$

Note that here the basic effect is the change of the logarithmic derivative of the growth function $f$, controlling the linear evolution of the velocity field, by the so called redshiftspace parameter

$$
\beta_{\mathrm{g}}(z):=\frac{f(z)}{b_{\mathrm{g}}(z)},
$$

controlling the strength of RSD on the fluctuations of tracers. Taking into account that $f$ controls the amplitude of the peculiar velocity field in linear theory, see section 1.2.1, the greater the velocity field, the greater the redshift space distortion effect and the greater the bias of some kind of tracers with respect to the total matter field, the lower the redshift space distortion effect. 


\section{Appendix D}

\section{Deflection of light rays by LSS in the Universe}

The present Appendix is devoted to the computation of the deflection of a light ray in the context of an up to first order perturbed FLRW Universe model as the basis of the weak lensing phenomena by large-scale structures in the Universe. During all the Appendix spatial coordinates $x^{i}=(r, \theta, \varphi)$ are choosen, where $r$ is the radial comoving distance (with dimension of length). We closely follow the treatment of chapter 7 in [3].

\section{D.1 Geometric optics approximation}

Astronomical observations interpretation is based on the geometric optics limit of Maxwell equations for the electromagnetic field, plus its quantum mechanical interpretation giving rise to the concept of photon. The description of propagation of electromagnetic radiation through a curved spacetime is done under this approximations.

Electromagnetic phenomena is described by the Faraday Tensor $F_{\mu \nu}$, an antisymmetric second rank tensor satisfying

$$
3 F_{[\mu \nu ; \sigma]}=F_{\mu \nu ; \alpha}+F_{\nu \alpha ; \mu}+F_{\alpha \mu ; \nu}=0,
$$

where the semicolon notation is used here to denote covariant derivative components and the square brackets denote total antisymmetrization process [14, 2]. The Faraday tensor can be defined in terms of a vector potential $A^{\mu}$ as its curl,

$$
F_{\mu \nu}=2 A_{[\mu ; \nu]}=A_{\mu ; \nu}-A_{\nu ; \mu},
$$

except for a gauge freedom $A_{\mu} \rightarrow A_{\mu}+\partial_{\mu} f$, with $f$ an arbitrary scalar field.

Electrodynamics follows from the electromagnetic action

$$
S_{\mathrm{em}}=-\frac{1}{4} \int \mathrm{d}^{4} x \sqrt{-g}\left[F^{\mu \nu} F_{\mu \nu}+A_{\mu} j^{\mu}\right]
$$

where $g:=\operatorname{det}\left[g_{\mu \nu}\right]$ and $j^{\mu}$ is a four-current describing the sources of electromagnetic field. The equations of motions associated with $S_{\mathrm{em}}$ are the Maxwell equations,

$$
\nabla_{\mu} F^{\mu \nu}=j^{\nu} .
$$

The propagation of electromagnetic radiation in vacuum obeys the source-free Maxwell equations,

$$
\nabla_{\mu} F^{\mu \nu}=0
$$


Taking advantage of the gauge freedom for $A^{\mu}$, one can fix the Lorentz gauge, $\nabla_{\mu} A^{\mu}=0$, and then use the commutation rule for covariant derivatives of a vector field in the form $[14]$

$$
R_{\sigma \mu \nu}^{\mu} A^{\sigma}=R_{\sigma \nu} A^{\sigma}=\left(\nabla_{\mu} \nabla_{\nu}-\nabla_{\nu} \nabla_{\mu}\right) A^{\mu}
$$

where $R_{\sigma \mu \nu}^{\mu}$ and $R_{\mu \nu}$ are the components of the Riemann and Ricci tensors, respectively; to obtain the equations of motion for $A^{\mu}$

$$
\nabla_{\mu} A^{\mu}=0, \quad \nabla_{\nu} \nabla^{\nu} A_{\mu}+R_{\mu \nu} A^{\nu}=0
$$

The geometric optics limit resides on the assumption of a solution to (D.7) of the form

$$
A_{\mu}=g(\psi) \alpha_{\mu}+\text { tail terms, }
$$

where (a) $g(\psi)$ is an arbitraty function of the phase of radiation $\psi$, (b) $g$ varies rapidly compared with the amplitude $\alpha_{\mu}$, in the following sense

$$
\left|g^{\prime} k_{[\mu} \alpha_{\nu]}\right| \gg\left|g \nabla_{[\mu} \alpha_{\nu]}\right|
$$

and (c) the tail terms are small compared with the first term in solution (D.8), i.e., the latter defines the dominant part of the solution. On equation (D.9) the propagation vector of electromagnetic radiation $k_{\mu}$ was introduced as the spacetime variation of the phase,

$$
k_{\mu}:=\nabla_{\mu} \psi
$$

Note that since the phase is a scalar field the propagation vector is curl-free, i.e.,

$$
k_{[\mu ; \nu]}=0 .
$$

The condition (a) tells that arbitrary information can be propagated by the signal, while (b) tells that solution (D.8) represents a high-frequency wave with relatively slow varying amplitude.

Substituting the geometric optics solution (D.8) on the vacuum Maxwell equations (D.7), ignoring the tail terms and equating to zero the coefficients of $g, g^{\prime}$ and $g^{\prime \prime}$, because $g$ is arbitrary according to (a), one obtains the equations

$$
\begin{aligned}
k_{\mu} \alpha^{\mu}=\nabla_{\mu} \alpha^{\mu} & =0, \\
k_{\mu} k^{\mu} & =0, \\
2 k^{\nu} \nabla_{\nu} \alpha^{\mu} & =0, \\
\nabla_{\nu} \nabla^{\nu} \alpha_{\mu}+R_{\mu \nu} \alpha^{\nu} & =0 .
\end{aligned}
$$

equation (D.12b) shows that $k^{\mu}$ is a null four-vector, therefore the first of (D.12a) shows that the amplitude $\alpha^{\mu}$ is a space-like four-vector. Taking the covariant derivative of (D.12b) one see that $\nabla_{\nu}\left(k_{\mu} k^{\mu}\right)=0$, so that $k^{\mu} \nabla_{\nu} k^{\mu}=0$, therefore, equation (D.11) implies

$$
k^{\mu} \nabla_{\mu} k^{\nu}=0 .
$$

This is, the integral curves of the propagation vector, say $x^{\mu}(s)$, i.e., those tangent to $k^{\mu}$, $k^{\mu}=\frac{\mathrm{d} x^{\mu}}{\mathrm{d} s}$, are null geodesics of the spacetime (here $s$ is an arbitrary affine parameter for the integral curves of $k^{\mu}$ ). 
By equation (D.12b) $k^{\mu} \nabla_{\mu} \psi=0$, i.e., the covariant derivative along the integral curves of $k^{\mu}, x^{\mu}(s)$, of the phase $\psi$ of radiation is null. This implies that $\psi\left(x^{\mu}(s)\right)=$ const, along this curves or equivalently $k^{\mu}$ is orthogonal to the wave surface, were $\psi=$ const.

The above results allow one to interpret the integral curves of $k^{\mu}$ as light-rays, i.e., photon trajectories on the spacetime. They are null geodesics as is expected by foundational principles of GR and the surfaces $\psi=$ const are the future ligh-cones of the emitter's world line $[22,14]$. This results are also the basis of gravitational lensing phenomena, they imply that light-rays are differentially bent by inhomogeneous gravitational fields, according to the null geodesic equation.

equation (D.12c) imply that the amplitude of radiation $\alpha^{\mu}$ is parallely propagated along light-rays, telling that the polarization state is non-affected by gravitational fields. Since $\alpha^{\mu}$ should be orthogonal to $k^{\mu}$ and space-like, there are only two degrees of freedom determined by (D.12c). They should be properly interpreted as determining the polarization of radiation.

\section{D.2 Background null geodesics}

The goal here is to solve the null geodesic equation,

$$
k^{\nu} \nabla_{\nu} k^{\mu}=0 ; \quad k^{\mu}=\frac{\mathrm{d} x^{\mu}}{\mathrm{d} s} ; \quad g_{\mu \nu} k^{\mu} k^{\nu}=0,
$$

on the RW metric, which accounts for the background model of FLRW Universe models.

Without loss of generality (because of the homogeneity and isotropy of space) one can assume that photon trajectory is radial, i.e., $\forall s \bar{x}^{2}(s)=\bar{x}^{3}(s)=0$. Then the geodesic equation (D.14) reduces to the following two equations

$$
\begin{aligned}
\frac{\mathrm{d}^{2} \eta}{\mathrm{d} s^{2}} & =-\bar{\Gamma}^{0}{ }_{\mu \nu} \frac{\mathrm{d} \bar{x}^{\mu}}{\mathrm{d} s} \frac{\mathrm{d} \bar{x}^{\nu}}{\mathrm{d} s}=-\bar{\Gamma}_{00}^{0}\left(\frac{\mathrm{d} \eta}{\mathrm{d} s}\right)^{2}-\bar{\Gamma}_{i j}^{0} \frac{\mathrm{d} \bar{x}^{i}}{\mathrm{~d} s} \frac{\mathrm{d} \bar{x}^{j}}{\mathrm{~d} s}=-\mathcal{H}\left[\left(\frac{\mathrm{d} \eta}{\mathrm{d} s}\right)^{2}+\left(\frac{\mathrm{d} r}{\mathrm{~d} s}\right)^{2}\right] \\
\frac{\mathrm{d}^{2} r}{\mathrm{~d} s^{2}} & =-\bar{\Gamma}_{\mu \nu}^{1} \frac{\mathrm{d} \bar{x}^{\mu}}{\mathrm{d} s} \frac{\mathrm{d} \bar{x}^{\nu}}{\mathrm{d} s}=-2 \bar{\Gamma}^{1}{ }_{0 i} \frac{\mathrm{d} \eta}{\mathrm{d} s} \frac{\mathrm{d} \bar{x}^{i}}{\mathrm{~d} s}-\bar{\Gamma}^{1}{ }_{i j} \frac{\mathrm{d} \bar{x}^{i}}{\mathrm{~d} s} \frac{\mathrm{d} \bar{x}^{j}}{\mathrm{~d} s}=-2 \mathcal{H} \frac{\mathrm{d} \eta}{\mathrm{d} s} \frac{\mathrm{d} r}{\mathrm{~d} s}
\end{aligned}
$$

together with the null--condition, which reduces to

$$
\left(\frac{\mathrm{d} \eta}{\mathrm{d} s}\right)^{2}=\left(\frac{\mathrm{d} r}{\mathrm{~d} s}\right)^{2}
$$

Combining this equations, one ends with the following equation for the conformal time coordinate of the photon's trajectory:

$$
\frac{\mathrm{d}^{2} \eta}{\mathrm{d} s^{2}}=-2 \mathcal{H}(\eta)\left(\frac{\mathrm{d} \eta}{\mathrm{d} s}\right)^{2}
$$

which can be solved by using the chain rule to write $\frac{\mathrm{d}^{2} \eta}{\mathrm{d} s^{2}}=-\left(\frac{\mathrm{d} \eta}{\mathrm{d} s}\right)^{3} \frac{\mathrm{d}^{2} s}{\mathrm{~d} \eta^{2}}$ in order to obtain

$$
\frac{\mathrm{d}^{2} s}{\mathrm{~d} \eta^{2}}=2 \mathcal{H}(\eta) \frac{\mathrm{d} s}{\mathrm{~d} \eta}
$$


Then, one ends up with the following relation between the conformal time $\eta$ and the affine parameter $s$ along the photon's trajectory:

$$
\mathrm{d} s=a^{2}(\eta) \mathrm{d} \eta
$$

For an incoming to the observer photon, the null condition, equation (D.14), tells that $\mathrm{d} \eta=-\mathrm{d} r$, so that by imposing the final conditions to the photon trajectory to arrive at the observer (assumed located at the origin, without loss of generality) at present conformal time $\eta_{0}$, the solution for the tangent vector to the photon trajectory are given by

$$
\bar{k}^{\mu}(s)=\frac{\mathrm{d} \bar{x}^{\mu}}{\mathrm{d} s}=a^{-2}(1,-\hat{\mathbf{n}}),
$$

where $\hat{\mathbf{n}}$ denotes a general unit vector defining the spatial direction of the photon. During this work, and without loss of generality, it only has radial component, i.e., $\hat{\mathbf{n}}=\partial_{r}$.

It is particularly useful to use the radial coordinate as affine parameter, note that this is possible because the solution for the radial coordinate is linear on the affine parameter,

$$
\bar{k}^{\mu}\left(x^{\nu}\right)=\bar{k}^{\mu}(r)=\frac{\mathrm{d} \bar{x}^{\mu}}{\mathrm{d} r}=(-1, \hat{\mathbf{n}}) .
$$

\section{D.3 First order perturbed null geodesics and deflec- tion angle}

Having the background solution for incoming trajectories of photons (light rays) given by equation (D.21) it is convenient to define first order perturbations of this trajectories by writing the photon geodesic as

$$
x^{\mu}(r)=\bar{x}^{\mu}(r)+\delta x^{\mu}(r),
$$

such that the tangent vector (choosing as affine parameter the radial comoving distance),

$$
k^{\mu}=\frac{\mathrm{d} x^{\mu}}{\mathrm{d} r}=(-1+\delta \nu, \hat{\mathbf{n}}+\delta \mathbf{e})
$$

where the fractional frequency perturbation, $\delta \nu:=\frac{\mathrm{d} \delta x^{0}}{\mathrm{~d} r}$, and the fractional perturbation to the photon momentum, $\delta \mathbf{e}^{i}:=\frac{\mathrm{d} \delta x^{i}}{\mathrm{~d} r}$, were introduced.

Since at the background level from equation (D.21) it is clear that $\bar{x}^{\mu}=\left(\eta_{0}-\right.$ $\left.r, r, \theta_{0}, \varphi_{0}\right)$, with $\theta_{0}$ and $\varphi_{0}$ constants that specify the position of the source (emission point) on the observer sky, one can write the perturbation to the photon trajectory as $\delta x^{\mu}=(\delta \eta(r), \delta r(r), \theta(r), \varphi(r))$. This is, the deflection of the photon trajectory, which is given by the angles $\theta(r)-\theta_{0}$ and $\varphi(r)-\varphi_{0}$, is directly related to the angular components of the fractional change in the photon momentum, which can be expanded as

$$
\delta \mathbf{e}=\delta e^{1} \partial_{r}+\frac{\mathrm{d} \theta}{\mathrm{d} r} \partial_{\theta}+\frac{\mathrm{d} \varphi}{\mathrm{d} r} \partial_{\varphi}
$$

Note that here the assumption that the unperturbed photon geodesic is radial was used.

The first order perturbation of the null geodesic equation (D.14) should determine the dynamics for the fractional frequency perturbation and the fractional perturbation to the 
photon momentum as long as it describes the perturbed photon trajectory (D.22).

$$
\begin{aligned}
\frac{\mathrm{d}}{\mathrm{d} r} \delta \nu & =-\delta \Gamma_{\mu \nu}^{0} \frac{\mathrm{d} \bar{x}^{\mu}}{\mathrm{d} r} \frac{\mathrm{d} \bar{x}^{\nu}}{\mathrm{d} r}-2 \bar{\Gamma}^{0}{ }_{\mu \nu} \frac{\mathrm{d} \bar{x}^{\mu}}{\mathrm{d} r} \frac{\mathrm{d} \delta x^{\nu}}{\mathrm{d} r} \\
\frac{\mathrm{d}}{\mathrm{d} r} \delta e^{i} & =-\delta \Gamma_{\mu \nu}^{i} \frac{\mathrm{d} \bar{x}^{\mu}}{\mathrm{d} r} \frac{\mathrm{d} \bar{x}^{\nu}}{\mathrm{d} r}-2 \bar{\Gamma}_{\mu \nu}^{i} \frac{\mathrm{d} \bar{x}^{\mu}}{\mathrm{d} r} \frac{\mathrm{d} \delta x^{\nu}}{\mathrm{d} r} .
\end{aligned}
$$

Working the equations (D.25) one can proof that

$$
\begin{aligned}
\delta \Gamma_{\mu \nu}^{0} \frac{\mathrm{d} \bar{x}^{\mu}}{\mathrm{d} r} \frac{\mathrm{d} \bar{x}^{\nu}}{\mathrm{d} r} & =\delta \Gamma_{00}^{0}\left(\frac{\mathrm{d} \eta}{\mathrm{d} r}\right)^{2}+2 \delta \Gamma_{01}^{0} \frac{\mathrm{d} \eta}{\mathrm{d} r}+\delta \Gamma_{11}^{0}{ }_{11} \\
& =\delta \Gamma_{00}^{0}-2 \delta \Gamma_{01}^{0}+\delta \Gamma^{0}{ }_{11} \\
& =(\Phi+\Psi)^{\prime}+2 \mathcal{H}(\Phi+\Psi)-\partial_{r} \Phi \\
\bar{\Gamma}_{\mu \nu}^{0} \frac{\mathrm{d} \bar{x}^{\mu}}{\mathrm{d} r} \frac{\mathrm{d} \delta x^{\nu}}{\mathrm{d} r}= & \bar{\Gamma}^{0}{ }_{00} \frac{\mathrm{d} \eta}{\mathrm{d} r} \delta \nu+\bar{\Gamma}_{0}^{0}{ }_{0 i} \frac{\mathrm{d} \eta}{\mathrm{d} r} \delta e^{i}+\bar{\Gamma}^{0}{ }_{10} \delta \nu+\bar{\Gamma}^{0}{ }_{1 j} \delta e^{j} \\
= & -\mathcal{H} \delta \nu+\mathcal{H} \delta e^{1}, \\
\delta \Gamma_{\mu \nu}^{i} \frac{\mathrm{d} \bar{x}^{\mu}}{\mathrm{d} r} \frac{\mathrm{d} \bar{x}^{\nu}}{\mathrm{d} r} & =\delta \Gamma_{00}^{i}\left(\frac{\mathrm{d} \eta}{\mathrm{d} r}\right)^{2}+2 \delta \Gamma_{01}^{i}\left(\frac{\mathrm{d} \eta}{\mathrm{d} r}\right)+\delta \Gamma^{i}{ }_{11} \\
& =\delta \Gamma^{i}{ }_{00}-2 \delta \Gamma_{01}^{i}+\delta \Gamma^{i}{ }_{11} \\
& =\gamma^{i j} \partial_{j}(\Phi+\Psi)+2 \gamma_{1}^{i} \Psi^{\prime}-2 \gamma_{1}^{i} \partial_{r} \Psi
\end{aligned}
$$

and

$$
\begin{aligned}
\bar{\Gamma}_{\mu \nu}^{i} \frac{\mathrm{d} \bar{x}^{\mu}}{\mathrm{d} r} \frac{\mathrm{d} \delta x^{\nu}}{\mathrm{d} r} & =\bar{\Gamma}^{i}{ }_{00} \frac{\mathrm{d} \eta}{\mathrm{d} r} \delta \nu+\bar{\Gamma}^{i}{ }_{0 j} \frac{\mathrm{d} \eta}{\mathrm{d} r} \delta e^{j}+\bar{\Gamma}^{i}{ }_{10} \delta \nu+\bar{\Gamma}^{i}{ }_{1 j} \delta e^{j} \\
& =-\mathcal{H} \delta e^{i}+\mathcal{H} \gamma_{1}^{i} \delta \nu+\bar{\Gamma}^{i}{ }_{1 j} \delta e^{j}
\end{aligned}
$$

so that

$$
\begin{aligned}
\frac{\mathrm{d}}{\mathrm{d} r} \delta \nu & =(\Phi+\Psi)^{\prime}-2 \mathcal{H}(\Phi+\Psi)+\partial_{r} \Phi+2 \mathcal{H} \delta \nu-2 \mathcal{H} \delta e^{1} \\
\frac{\mathrm{d}}{\mathrm{d} r} \delta e^{i} & =-\gamma^{i j} \partial_{j}(\Phi+\Psi)-2 \gamma_{1}^{i} \Psi^{\prime}+2 \gamma_{1}^{i} \partial_{r} \Psi+2 \mathcal{H} \delta e^{i}-2 \mathcal{H} \gamma_{1}^{i} \delta \nu-2 \bar{\Gamma}^{i}{ }_{1 j} \delta e^{j}
\end{aligned}
$$

Since the interest here is on solve for $\theta(r)$ and $\varphi(r)$, one can consider only the $i=2,3$ equations on (D.26). Begin by see that

$$
\begin{aligned}
& \bar{\Gamma}^{2}{ }_{1 j} \delta e^{j}=\bar{\Gamma}^{2}{ }_{11} \delta e^{1}+\bar{\Gamma}^{2}{ }_{12} \delta e^{2}+\bar{\Gamma}^{2}{ }_{13} \delta e^{3}=\bar{\Gamma}^{2}{ }_{12} \delta e^{2}=\frac{\partial_{r} f_{K}(r)}{f_{K}(r)} \frac{\mathrm{d} \theta}{\mathrm{d} r} \\
& \bar{\Gamma}^{3}{ }_{1 j} \delta e^{j}=\bar{\Gamma}^{3}{ }_{11} \delta e^{1}+\bar{\Gamma}^{3}{ }_{12} \delta e^{2}+\bar{\Gamma}^{3}{ }_{13} \delta e^{3}=\bar{\Gamma}^{3}{ }_{13} \delta e^{3}=\frac{\partial_{r} f_{K}(r)}{f_{K}(r)} \frac{\mathrm{d} \varphi}{\mathrm{d} r}
\end{aligned}
$$

so that, by nothing that all terms involving $\gamma_{1}^{i}$ on equation (D.26b) should vanish for the angular components $i=2,3$, one ends up with the equations

$$
\begin{aligned}
& \frac{\mathrm{d}^{2} \theta}{\mathrm{d} r^{2}}=-\frac{2}{f_{K}} \partial_{\theta} \Psi_{W}+2 \frac{\partial_{r} f_{K}}{f_{K}} \frac{\mathrm{d} \theta}{\mathrm{d} r}, \\
& \frac{\mathrm{d}^{2} \varphi}{\mathrm{d} r^{2}}=-\frac{2}{f_{K} \sin ^{2}(\theta)} \partial_{\varphi} \Psi_{W}+2 \frac{\partial_{r} f_{K}}{f_{K}} \frac{\mathrm{d} \varphi}{\mathrm{d} r},
\end{aligned}
$$


where the lensing or Weyl gravitational potential was introduced according to ${ }^{1}$

$$
\Psi_{W}:=\frac{1}{2}(\Phi+\Psi)
$$

The equations (D.27) can be written on a more symmetric way as

$$
\begin{aligned}
-\frac{\mathrm{d}}{\mathrm{d} r}\left(f_{K}^{2} \frac{\mathrm{d} \theta}{\mathrm{d} r}\right) & =2 \partial_{\theta} \Psi_{W} \\
-\frac{\mathrm{d}}{\mathrm{d} r}\left(f_{K}^{2} \frac{\mathrm{d} \varphi}{\mathrm{d} r}\right) & =2 \sin ^{-2}(\theta) \partial_{\varphi} \Psi_{W} .
\end{aligned}
$$

Integrating along the photon trajectory, from some arbitrary $r$ to the observer's position $r_{0}=0$ one have

$$
\begin{aligned}
& f_{K}^{2}(r) \frac{\mathrm{d} \theta}{\mathrm{d} r}(r)-f_{K}^{2}\left(r_{0}\right) \frac{\mathrm{d} \theta}{\mathrm{d} r}\left(r_{0}\right)=-2 \int_{r}^{r_{0}} \mathrm{~d} r^{\prime} \partial_{\theta} \Psi_{W}\left(x^{\mu}\left(r^{\prime}\right)\right) \\
& f_{K}^{2}(r) \frac{\mathrm{d} \varphi}{\mathrm{d} r}(r)-f_{K}^{2}\left(r_{0}\right) \frac{\mathrm{d} \varphi}{\mathrm{d} r}\left(r_{0}\right)=-2 \int_{r}^{r_{0}} \frac{\mathrm{d} r^{\prime}}{\sin ^{2}(\theta)} \partial_{\varphi} \Psi_{W}\left(x^{\mu}\left(r^{\prime}\right)\right)
\end{aligned}
$$

In order to compute the integrals on the right-hand side of (D.30) the Born approximation is introduced. This is, the integration will be performed at a first approximation over the unperturbed photon trajectory solution, equation (D.21). This assumption decouples the angular components of the first order perturbation to the null geodesic equation from its corresponding radial and temporal counterparts, expressed on the full system (D.26).

$$
\begin{aligned}
f_{K}^{2}(r) \frac{\mathrm{d}}{\mathrm{d} r} \theta(r) & =-2 \int_{r}^{r_{0}} \mathrm{~d} r \partial_{\theta} \Psi_{W}\left(\bar{x}^{\mu}(r)\right)=2 \int_{r}^{r_{0}} \mathrm{~d} r^{\prime} \partial_{\theta} \Psi_{W}\left(\eta_{0}-r^{\prime}, r^{\prime}, \theta_{0}, \varphi_{0}\right) \\
& =-2 \int_{r_{0}}^{r} \mathrm{~d} r^{\prime} \partial_{\theta} \Psi_{W}\left(\eta_{0}-r^{\prime}, r^{\prime}, \theta_{0}, \varphi_{0}\right),
\end{aligned}
$$

and

$$
f_{K}^{2}(r) \frac{\mathrm{d}}{\mathrm{d} r} \varphi(r)=-2 \int_{r_{0}}^{r} \frac{\mathrm{d} r^{\prime}}{\sin ^{2} \theta_{0}} \partial_{\varphi} \Psi_{W}\left(\eta_{0}-r^{\prime}, r^{\prime}, \theta_{0}, \varphi_{0}\right)
$$

remember that here $x_{0}^{\mu}=\left(\eta_{0}, r_{0}=0, \theta_{0}, \varphi_{0}\right)$ are the coordinates of the photon reception event. Integrating one more time this equations we can arrive to

$$
\begin{aligned}
& \theta\left(r_{\mathrm{s}}\right)=\theta_{0}-2 \int_{0}^{r_{\mathrm{s}}} \mathrm{d} r \frac{f_{K}\left(r_{\mathrm{s}}-r\right)}{f_{K}\left(r_{\mathrm{s}}\right) f_{K}(r)} \partial_{\theta} \Psi_{W}\left(\eta_{0}-r, r, \theta_{0}, \varphi_{0}\right) \\
& \varphi\left(r_{\mathrm{s}}\right)=\varphi_{0}-\frac{2}{\sin ^{2} \theta_{0}} \int_{0}^{r_{\mathrm{s}}} \mathrm{d} r \frac{f_{K}\left(r_{\mathrm{s}}-r\right)}{f_{K}\left(r_{\mathrm{s}}\right) f_{K}(r)} \partial_{\varphi} \Psi_{W}\left(\eta_{0}-r, r, \theta_{0}, \varphi_{0}\right),
\end{aligned}
$$

where it was introduced the subindex "s" notation to refers the source (emission of the light ray) coordinates.

\footnotetext{
${ }^{1}$ The name comes from the fact that this potential appears as responsible for the linear perturbations of the Weyl tensor, see [3].
} 
In order to proof that the expressions (D.32) are in fact first integrals of (D.31) one can simply evaluate the derivative

$$
\begin{aligned}
\frac{\mathrm{d}}{\mathrm{d} r_{\mathrm{s}}} \int_{0}^{r_{\mathrm{s}}} \mathrm{d} r \frac{f_{K}\left(r_{\mathrm{s}}-r\right)}{f_{K}\left(r_{\mathrm{s}}\right) f_{K}(r)} F(r, 0)= & \int_{0}^{r_{\mathrm{s}}} \\
& \mathrm{d} r \frac{f_{K}\left(r_{\mathrm{s}}\right) f_{K}^{\prime}\left(r_{\mathrm{s}}-r\right)-f_{K}\left(r_{\mathrm{s}}-r\right) f_{K}^{\prime}\left(r_{\mathrm{s}}\right)}{f_{K}\left(r_{\mathrm{s}}\right)^{2} f_{K}(r)} F(r, 0) \\
& +\frac{f_{K}(0)}{f_{K}(x)^{2}} F(r, 0)
\end{aligned}
$$

then, the result is a consequence of the following identity

$$
f_{K}\left(r_{\mathrm{s}}\right) f_{K}^{\prime}\left(r_{\mathrm{s}}-r\right)-f_{K}\left(r_{\mathrm{s}}-r\right) f_{K}^{\prime}\left(r_{\mathrm{s}}\right)=f_{K}(r)
$$

valid for all values of the spatial curvature $K$ and the corresponding ranges of the radial comoving distance, $r, r_{\mathrm{s}} \in[0, \infty)$ for $K \leq 0$ and $\left(r_{\mathrm{s}}-r\right) \in\left[0, K^{1 / 2} \pi\right]$ for $K>0$; and follows from the definition of $f_{K}(r)$, equation (1.7).

The solutions (D.32) allows to conclude that the deflection angle of a light ray in the Born approximation in the Universe is given by

$$
\boldsymbol{\alpha}:=\left(\theta-\theta_{0}, \varphi-\varphi_{0}\right)=-2 \int_{0}^{r_{\mathrm{s}}} \mathrm{d} r \frac{f_{K}\left(r_{\mathrm{s}}-r\right)}{f_{K}\left(r_{\mathrm{s}}\right) f_{K}(r)} \nabla_{\perp} \Psi_{W}\left(\eta_{0}-r, r, \theta_{0}, \varphi_{0}\right),
$$

where $\nabla_{\perp}:=\left(\partial_{\theta},\left(\sin \theta_{0}\right)^{-2} \partial_{\varphi}\right)$ is the covariant gradient on the unit sphere $\mathbb{S}_{2}$. 


\section{Appendix E}

\section{FFTLog method}

The present appendix review the construction of the FFTLog algorithm used to compute the distant observer multipoles of the two-point correlation function, equation (4.35). We closely follow the original treatment of [74].

First, a very brief review of the basic ingredients of a discrete Fourier transform for a sequence of linearly spaced points are considered. Then, in an analogous way, the discrete Hankel transform equations (4.57) is presented for a sequence of logarithmically spaced points. Thus, it is showed that the main point on the FFTLog method is the fact that the intrgration on the Hankel transform definition is translated to the computation of the Mellin transform of the Bessel function of the first kind, which is finally showed to be analytically given in terms of the Gamma function.

Since there exists a good numerical approximation for the Gamma function, namely the Lanczos approximation [73], the FFTLog algorithm can be constructed on the basis of a Fast Fourier Transform [75] and an implementation of such approximation according to the pseudocode on Algorithm 4.

\section{E.1 Discrete Fourier transform}

Given a periodic and analytic function $a: \mathbb{R} \rightarrow \mathbb{R}$ with period $R$, i.e., $a(r+R)=a(r)$, the periodicity property ensures that its continuous Fourier transform should contain only discrete Fourier modes of the form [73, 37]

$$
\exp \left[2 \pi i m \frac{r}{R}\right] ; \quad m \in \mathbb{Z}
$$

Consider as the fundamental interval where $a$ is defined the interval $[-R / 2, R / 2]$ and suppose further that $a$ is smooth in the sense that can be expanded by a finite number of modes, the lowest $N$ (assumed even here for simplicity),

$$
a(r)=\sum_{m}^{\prime} c_{m} \exp \left[2 \pi i m \frac{r}{R}\right]
$$

with $c_{-N / 2}=c_{N / 2}$. Here the following notation for the summation was introduced

$$
\sum_{m}^{\prime} x_{m}:=\sum_{m=-N / 2}^{N / 2} w_{m} x_{m} ; \quad w_{m}:= \begin{cases}1 / 2, & m= \pm N / 2 \\ 1, & \text { otherwise }\end{cases}
$$


The Sampling Theorem [73] states that given a function $a$ with the described properties and satisfying the relation (E.2) its Fourier coefficients are given by

$$
c_{m}=\frac{1}{N} \sum_{n}^{\prime} a_{n} \exp \left[-2 \pi i m \frac{n}{N}\right] ; \quad a_{n}:=a\left(r_{n}\right), \quad r_{n}:=\frac{n R}{N} .
$$

Where the discrete values $\left\{a_{n}\right\}$ themselves satisfy

$$
a_{n}=\sum_{m}^{\prime} c_{m} \exp \left[2 \pi i m \frac{n}{N}\right] .
$$

Equations (E.4) and (E.5) constitute the discrete Fourier transform pair that relates the two periodic and linearly spaced sequences $\left\{a_{n}\right\}$ and $\left\{c_{n}\right\}$ of length $N$ and they are the basis of standard FFT methods. [75]

\section{E.2 Discrete Hankel transform and FFTLog method}

Consider a function $a: \mathbb{R} \rightarrow \mathbb{R}$ analytic and periodic in logarithmic space with period $L$, i.e.,

$$
a\left(r e^{L}\right)=a(r) .
$$

In analogy with the discrete Fourier transform, let us consider a fundamental interval as $\left[\ln \left(r_{0}\right)-L / 2, \ln \left(r_{0}\right)+L / 2\right]$. Note that $r_{0}$, the central point of the interval, is left as a free parameter. The periodicity of the function imply that its continuous Fourier transform should contain only discrete Fourier modes. Now suppose further that the function is smooth in the sense that it only contains the lowest $N$ (assumed even for simplicity) Fourier modes,

$$
a(r)=\sum_{m}^{\prime} c_{m} \exp \left[2 \pi i m \frac{\ln \left(r / r_{0}\right)}{L}\right],
$$

where $c_{-N / 2}=c_{N / 2}$. Then, the Sampling Theorem [73] guarantees that the coefficients $c_{m}$ satisfy

$$
c_{m}=\frac{1}{N} \sum_{n}^{\prime} a_{n} \exp \left[-2 \pi i m \frac{n}{N}\right] ; \quad a_{n}:=a\left(r_{n}\right), \quad r_{n}:=r_{0} \exp \left[L \frac{n}{N}\right] .
$$

Consider now the Hankel transform, equation (4.57), of $a(r), \tilde{a}(k)$. Since $a$ is given by the expansion (E.7) $\tilde{a}$ can be written as

$$
\tilde{a}(k)=\sum_{m}^{\prime} c_{m} \int_{0}^{\infty} \mathrm{d} r k(k r)^{q} J_{\mu}(k r) \exp \left[2 \pi i m \frac{\ln \left(r / r_{0}\right)}{L}\right],
$$

so that, rewriting the exponential inside the integrand as

$$
\exp \left[2 \pi i m \frac{\ln \left(r / r_{0}\right)}{L}\right]=r_{0}^{-2 \pi i m / L} r^{2 \pi i m / L}
$$

and introducing the change of variables $t:=k r$ one has

$$
\tilde{a}(k)=\sum_{m}^{\prime} c_{m}\left(k r_{0}\right)^{-2 \pi i m / L}\left[\int_{0}^{\infty} \mathrm{d} t t^{(q+2 \pi i m / L)} J_{\mu}(t)\right] .
$$


Now, by introducing $k_{0}$ in order to write

$$
k^{-2 \pi i m / L}=k_{0}^{-2 \pi i m / L} \exp \left[-2 \pi i m \frac{\ln \left(k / k_{0}\right)}{L}\right]
$$

one finally arrives to

$$
\tilde{a}(k)=\sum_{m}^{\prime} c_{m} u_{m} \exp \left[-2 \pi i m \frac{\ln \left(k / k_{0}\right)}{L}\right],
$$

where the coefficients $u_{m}$ were introduced as

$$
\begin{aligned}
u_{m}(\mu, q) & :=\left(k_{0} r_{0}\right)^{-2 \pi i m / L} U_{\mu}(q+2 \pi i m / L) \\
& :=\left(k_{0} r_{0}\right)^{-2 \pi i m / L} \int_{0}^{\infty} \mathrm{d} t t^{(q+2 \pi i m / L)} J_{\mu}(t),
\end{aligned}
$$

where the complex-valued function $U_{\mu}$ was introduced as

$$
U_{\mu}(x):=\int_{0}^{\infty} \mathrm{d} t t^{x} J_{\mu}(t)
$$

The key point of the FFTLog method lies here, on the function $U_{\mu}(x)$. The first thing to note is that it is the Mellin transform [37] of the Bessel function of the first kind $J_{\mu}$, $U_{\mu}(x)=\mathcal{M}\left[J_{\mu}\right](x+1)$. Such a Mellin transform can be computed analytically in terms of the Gamma function $\Gamma$. The explicit computation is presented on $\S \mathrm{E} .3$ and allows one to write, equation (E.29),

$$
U_{\mu}(x)=2^{x} \frac{\Gamma[(1+\mu+x) / 2]}{\Gamma[(1+\mu-x) / 2]} .
$$

Thus one ends up with an expansion for the Hankel transform of $a(r)$ in the form of a Fourier expansion. Note that the functions $u_{m}(\mu, q)$ satisfy

$$
u_{m}^{*}=u_{-m},
$$

property that guarantees that if $a(r)$ is real-valued, its Hankel transform is also realvalued. Also, in the development, the variable $k_{0}$ was introduced, it should be understood as the center of the fundamental interval where the Hankel transform $\tilde{a}(k)$ is defined, $\left[\ln \left(k_{0}\right)-L / 2, \ln \left(k_{0}\right)+L / 2\right]$.

In the following, the goal is to derive a sequence of discrete samples for the Hankel transform $\tilde{a}(k)$ at the discrete points $k_{0} e^{L n / N}$, in analogy to the discrete Fourier transform. By looking at the expansion (E.11) one can think in use the Sampling theorem [73] to complete this goal. However, the sampling theorem requires the outermost coefficintent on the expansion to be equal, i.e., $c_{-N / 2} u_{-N / 2}=c_{N / 2} u_{N / 2}$. From construction, $c_{-N / 2}=c_{N / 2}$ so the condition reduces to

$$
u_{-N / 2}=u_{N / 2}
$$

This condition can not be guaranteed only from the definition of the $u_{m}$ coefficients, equations (E.12). however, one can see that the sum of the terms with $m=-N / 2$ and $m=N / 2$ evaluated at the discrete points $k_{n}=k_{0} e^{L n / N}$ is given by [74]

$$
(-1)^{n} c_{N / 2}\left(u_{N / 2}-u_{-N / 2}\right)=(-1)^{n} c_{N / 2}\left(u_{N / 2}+u_{N / 2}^{*}\right)=(-1)^{n} c_{N / 2} 2 \Re\left[u_{N / 2}\right],
$$


so that the expansion (E.11) remains valid for all discrete points $k_{n}=k_{0} e^{\operatorname{Ln} / N}$ if one replaces

$$
u_{ \pm N / 2} \rightarrow \Re\left[u_{N / 2}\right]
$$

This replacement guarantees the condition (E.14) and therefore sampling theorem asserts that the coefficients $c_{m} u_{m}$ in the expansion (E.11) of the Hankel transform of $a(r)$ at the $N$ discrete points $\left\{k_{n}=k_{0} e^{L n / N} \mid n \in\{-N / 2,-N / 2+1, \ldots, N / 2\}\right\}$ determine a discrete Fourier pair with the sequence $\tilde{a}_{n}=\tilde{a}\left(k_{n}\right)$, i.e.

$$
\begin{gathered}
c_{m} u_{m}=\frac{1}{N} \sum_{n}^{\prime} \tilde{a}_{n} \exp \left[2 \pi i m \frac{n}{N}\right], \\
\tilde{a}_{n}=\sum_{m}^{\prime} c_{m} u_{m} \exp \left[-2 \pi i m \frac{n}{N}\right] .
\end{gathered}
$$

By combining the equations (E.8) and (E.16) we can arrive to the discrete Hankel transform pair

$$
\begin{aligned}
& a_{n}=\sum_{m}^{\prime} \tilde{a}_{m}\left\{\frac{1}{N} \sum_{k}^{\prime} \frac{1}{u_{k}} \exp \left[2 \pi i k \frac{n+m}{N}\right]\right\}, \\
& \tilde{a}_{m}=\sum_{n}^{\prime} a_{n}\left\{\frac{1}{N} \sum_{k}^{\prime} u_{k} \exp \left[2 \pi i k \frac{n+m}{N}\right]\right\},
\end{aligned}
$$

The discrete Hankel transform pair in the form of equations (E.16) constitutes the basis of the FFTLog algorithm [74] which is summarized in the form of pseudocode on Algorithm 4. Note that the second line on Algorithm 4, the key result in the FFTLog method, can be accomplished in practice by using a numerical routine for the Gamma function $\Gamma$. A well known and well behaved routine for $\Gamma$, and the one implemented on this work, is the Lanczos approximation, see e.g. $\S 6.1$ of [73].

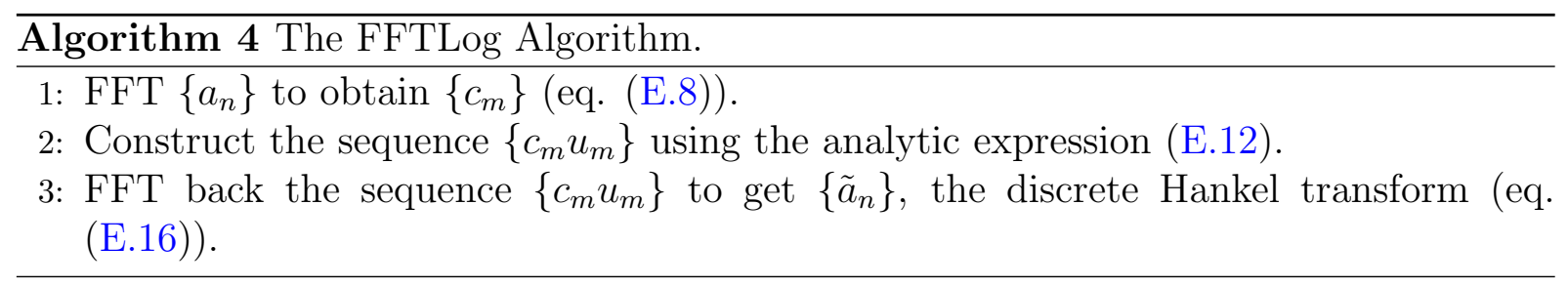

\section{E.3 Mellin transform of Bessel functions of the first kind}

The Mellin transform of a function $f$ is defined as the integral transform [37]

$$
\mathcal{M}[f](s):=\int_{0}^{\infty} \mathrm{d} z z^{s-1} f(z)
$$

The goal of the present section is to compute the Mellin transform of first kind Bessel functions $J_{\mu}(z)$ and particularly, to show the basic relation in which the FFTLog method rests, equation (E.13). 
Begin with the integral representation for $J_{\mu}$

$$
J_{\mu}(z)=\frac{2^{1-\mu}}{\sqrt{\pi} \Gamma\left(\mu+\frac{1}{2}\right)} z^{\mu} \int_{0}^{1} \mathrm{~d} t\left(1-t^{2}\right)^{\mu-\frac{1}{2}} \cos (z t), \quad \forall z \in \mathbb{C} \quad \text { and } \quad \forall \Re(\mu)>-1 .
$$

This relation is a direct consequence of the Sonin integral relations, see equations (14.171)(14.174) of [100].

One can then interchange the two occurring integrals in the Mellin transform computation to get

$$
\mathcal{M}\left[J_{\mu}\right](s)=\frac{2^{1-\mu}}{\sqrt{\pi} \Gamma\left(\mu+\frac{1}{2}\right)} \int_{0}^{1} \mathrm{~d} t\left(1-t^{2}\right)^{\mu-\frac{1}{2}} \int_{0}^{\infty} \mathrm{d} z z^{\mu+s-1} \cos (z t) .
$$

The external integral is the Mellin transform of the trigonometric cosine function [37],

$$
\mathcal{M}[\cos (a x)](s):=\int_{0}^{\infty} \mathrm{d} x x^{s-1} \cos (a x)=a^{-s} \Gamma(s) \cos \left(\frac{\pi s}{2}\right),
$$

valid $\forall a>0$ and $\forall 0<\Re(s)<1$. Therefore, as in (E.20) by construction $t>0$, the only restriction that should be done is $0<\Re(\mu+s)<1$ to get

$$
\mathcal{M}\left[J_{\mu}\right](s)=\frac{2^{1-\mu}}{\sqrt{\pi} \Gamma\left(\mu+\frac{1}{2}\right)} \cos \left[(\mu+s) \frac{\pi}{2}\right] \Gamma(\mu+s) \int_{0}^{1} \mathrm{~d} t \frac{\left(1-t^{2}\right)^{\mu-\frac{1}{2}}}{t^{\mu+s}} .
$$

The integral in $t$ can be evaluated by introducing the change of variable $\sin (\vartheta):=t$ for $\vartheta \in\left[0, \frac{\pi}{2}\right]$. In fact, by using the representation of the beta function, see e.g., $§ 7.4$ of $[100]$,

$$
B(p, q)=2 \int_{0}^{\frac{\pi}{2}} \mathrm{~d} \vartheta \cos ^{2 p-1}(\vartheta) \sin ^{2 q-1}(\vartheta), \quad \forall \Re(p), \Re(q)>0,
$$

it is possible to write

$$
\begin{aligned}
\int_{0}^{1} \mathrm{~d} t \frac{\left(1-t^{2}\right)^{\mu-\frac{1}{2}}}{t^{\mu+s}} & =\int_{0}^{\frac{\pi}{2}} \mathrm{~d} \vartheta \cos ^{2 \mu}(\vartheta) \sin ^{-\mu-s}(\vartheta)=\frac{1}{2} B\left(\mu+\frac{1}{2}, \frac{-\mu-s+1}{2}\right) \\
& =\frac{\Gamma\left(\mu+\frac{1}{2}\right) \Gamma\left(\frac{1-\mu-s}{2}\right)}{2 \Gamma\left(\frac{2+\mu-s}{2}\right)}
\end{aligned}
$$

under the restrictions $\Re(\mu)>-\frac{1}{2}$ and $\Re(s+\mu)<1$, which together with the restriction of equation (E.22) can be simplified to $-\Re(\mu)<\Re(s)<\frac{3}{2}$.

Thus,

$$
\mathcal{M}\left[J_{\mu}\right](s)=\frac{\cos \left[(\mu+s) \frac{\pi}{2}\right]}{\sqrt{\pi} 2^{\mu}} \frac{\Gamma(\mu+s) \Gamma\left(\frac{1-\mu-s}{2}\right)}{\Gamma\left(\frac{2+\mu-s}{2}\right)} .
$$

In order to simplify this expression and leave it in terms of Gamma functions only, one can use the Euler reflection formula on the form (see e.g., §7.4.1 [100])

$$
\frac{\cos (\pi z)}{\pi}=\frac{1}{\Gamma\left(\frac{1}{2}-z\right) \Gamma\left(\frac{1}{2}+z\right)}
$$

to write

$$
\cos \left[(\mu+s) \frac{\pi}{2}\right]=\frac{\pi}{\Gamma\left(\frac{1-\mu-s}{2}\right) \Gamma\left(\frac{1+\mu+s}{2}\right)} ;
$$


and the Legendre duplication formula (see e.g., $§ 7.4 .2$ from [100]),

$$
\Gamma(z) \Gamma\left(z+\frac{1}{2}\right)=2^{1-2 z} \sqrt{\pi} \Gamma(2 z)
$$

with $z=(\mu+s) / 2$ to write

$$
\Gamma(\mu+s)=\frac{2^{\mu+s-1}}{\sqrt{\pi}} \Gamma\left(\frac{\mu+s+1}{2}\right) \Gamma\left(\frac{\mu+s}{2}\right)
$$

Then, inserting (E.26) and (E.28) into (E.24) one ends up with the desired result

$$
\mathcal{M}\left[J_{\mu}\right](s)=2^{s-1} \frac{\Gamma\left(\frac{\mu+s}{2}\right)}{\Gamma\left(\frac{2+\mu-s}{2}\right)}, \quad \forall-\Re(\mu)<\Re(s)<\frac{3}{2} .
$$




\section{Bibliography}

[1] A. Albrecht, G. Bernstein, R. Cahn, W. L. Freedman, J. Hewitt, W. Hu, J. Huth, M. Kamionkowski, E. W. Kolb, L. Knox, J. C. Mather, S. Staggs, and N. B. Suntzeff, Report of the Dark Energy Task Force, ArXiv Astrophysics e-prints (Sept., 2006) [astro-ph/0609591].

[2] P. Peter and J.-P. Uzan, Primordial Cosmology. Oxford University Press, 2013.

[3] R. Durrer, The Cosmic Microwave Background. Cambridge University Press, Sept., 2008.

[4] S. Dodelson, Modern cosmology. Academic Press, 2003.

[5] G. F. R. Ellis and H. van Elst, Cosmological Models (Cargèse lectures 1998), in NATO ASIC Proc. 541: Theoretical and Observational Cosmology (M. Lachièze-Rey, ed.), pp. 1-116, 1999. gr-qc/9812046.

[6] F. Montesano, The full shape of the large-scale galaxy power spectrum: modelling and cosmological implications. PhD thesis, Ludwig-Maximilians-Universität, 2011.

[7] F. Sobreira, F. de Simoni, R. Rosenfeld, L. A. N. da Costa, M. A. G. Maia, and M. Makler, Cosmological forecasts from photometric measurements of the angular correlation function, Physical Review D 84 (Nov., 2011) 103001, [arXiv: 1109.4955].

[8] M. Crocce, A. Cabré, and E. Gaztañaga, Modelling the angular correlation function and its full covariance in photometric galaxy surveys, Monthly Notices of the Royal Astronomical Society 414 (June, 2011) 329-349, [arXiv: 1004.4640].

[9] F. Sobreira, Estimativas de parâmetros cosmológicos para o Dark Energy Survey. $\mathrm{PhD}$ thesis, Instituto de Física Teórica. Universidade Estadual Paulista, 2011.

[10] M. Bartelmann, TOPICAL REVIEW Gravitational lensing, Classical and Quantum Gravity 27 (Dec., 2010) 233001, [arXiv:1010.3829].

[11] M. Bartelmann and P. Schneider, Weak gravitational lensing, Physics Reports 340 (Jan., 2001) 291-472, [astro-ph/9912508].

[12] F. de Simoni, F. Sobreira, A. Carnero, A. J. Ross, H. O. Camacho, R. Rosenfeld, M. Lima, L. A. N. da Costa, and M. A. G. Maia, Large-scale analysis of the SDSS-III DR8 photometric luminous galaxies angular correlation function, Monthly Notices of the Royal Astronomical Society 435 (Nov., 2013) 3017-3027, [arXiv:1308.0630]. 
[13] P. Fosalba, E. Gaztañaga, F. J. Castander, and M. Manera, The onion universe: all sky lightcone simulations in spherical shells, Monthly Notices of the Royal Astronomical Society 391 (Nov., 2008) 435-446, [arXiv:0711.1540].

[14] S. Weinberg, Gravitation and Cosmology: Principles and Applications of the General Theory of Relativity. John Wiley \& Sons, July, 1972.

[15] A. G. Riess, A. V. Filippenko, P. Challis, A. Clocchiatti, A. Diercks, P. M. Garnavich, R. L. Gilliland, C. J. Hogan, S. Jha, R. P. Kirshner, B. Leibundgut, M. M. Phillips, D. Reiss, B. P. Schmidt, R. A. Schommer, R. C. Smith, J. Spyromilio, C. Stubbs, N. B. Suntzeff, and J. Tonry, Observational Evidence from Supernovae for an Accelerating Universe and a Cosmological Constant, The Astronomical Journal 116 (Sept., 1998) 1009-1038, [astro-ph/9805201].

[16] S. Perlmutter, G. Aldering, G. Goldhaber, R. A. Knop, P. Nugent, P. G. Castro, S. Deustua, S. Fabbro, A. Goobar, D. E. Groom, I. M. Hook, A. G. Kim, M. Y. Kim, J. C. Lee, N. J. Nunes, R. Pain, C. R. Pennypacker, R. Quimby, C. Lidman, R. S. Ellis, M. Irwin, R. G. McMahon, P. Ruiz-Lapuente, N. Walton, B. Schaefer, B. J. Boyle, A. V. Filippenko, T. Matheson, A. S. Fruchter, N. Panagia, H. J. M. Newberg, W. J. Couch, and Supernova Cosmology Project, Measurements of Omega and Lambda from 42 High-Redshift Supernovae, The Astrophysical Journal 517 (June, 1999) 565-586, [astro-ph/9812133].

[17] Planck Collaboration, P. A. R. Ade, N. Aghanim, C. Armitage-Caplan, M. Arnaud, M. Ashdown, F. Atrio-Barandela, J. Aumont, C. Baccigalupi, A. J. Banday, and et al., Planck 2013 results. XVI. Cosmological parameters, ArXiv e-prints (Mar., 2013) [arXiv:1303.5076].

[18] L. Amendola and S. Tsujikawa, Dark Energy: Theory and Observations. Cambridge University Press, 2010.

[19] P. J. Peebles and B. Ratra, The cosmological constant and dark energy, Reviews of Modern Physics 75 (Apr., 2003) 559-606, [astro-ph/0207347].

[20] E. J. Copeland, M. Sami, and S. Tsujikawa, Dynamics of Dark Energy, International Journal of Modern Physics D 15 (2006) 1753-1935, [hep-th/0603057].

[21] S. Tsujikawa, Modified Gravity Models of Dark Energy, in Lecture Notes in Physics, Berlin Springer Verlag (G. Wolschin, ed.), vol. 800 of Lecture Notes in Physics, Berlin Springer Verlag, pp. 99-145, Mar., 2010. arXiv:1101.0191.

[22] R. M. Wald, General relativity. University of Chicago Press, 1984.

[23] J. M. Bardeen, Gauge-invariant cosmological perturbations, Physical Review D 22 (Oct., 1980) 1882-1905.

[24] C.-P. Ma and E. Bertschinger, Cosmological Perturbation Theory in the Synchronous and Conformal Newtonian Gauges, The Astrophysical Journal 455 (Dec., 1995) 7, [astro-ph/9506072]. 
[25] M. Bruni, S. Matarrese, S. Mollerach, and S. Sonego, Perturbations of spacetime: gauge transformations and gauge invariance at second order and beyond, Classical and Quantum Gravity 14 (Sept., 1997) 2585-2606, [gr-qc/9609040].

[26] P. J. E. Peebles, The large-scale structure of the universe. Princeton University Press, 1980.

[27] F. Bernardeau, S. Colombi, E. Gaztañaga, and R. Scoccimarro, Large-scale structure of the Universe and cosmological perturbation theory, Physics Reports 367 (Sept., 2002) 1-248, [astro-ph/0112551].

[28] A. Lewis and S. Bridle, Cosmological parameters from CMB and other data: A Monte Carlo approach, Physical Review D 66 (Nov., 2002) 103511, [astro-ph/0205436].

[29] M. Crocce and R. Scoccimarro, Renormalized cosmological perturbation theory, Physical Review D 73 (Mar., 2006) 063519, [astro-ph/0509418].

[30] M. Crocce and R. Scoccimarro, Nonlinear evolution of baryon acoustic oscillations, Physical Review D 77 (Jan., 2008) 023533, [arXiv:0704.2783].

[31] J. M. Bardeen, J. R. Bond, N. Kaiser, and A. S. Szalay, The statistics of peaks of Gaussian random fields, The Astrophysical Journal 304 (May, 1986) 15-61.

[32] N. Padmanabhan, D. J. Schlegel, U. Seljak, A. Makarov, N. A. Bahcall, M. R. Blanton, J. Brinkmann, D. J. Eisenstein, D. P. Finkbeiner, J. E. Gunn, D. W. Hogg, Ž. Ivezić, G. R. Knapp, J. Loveday, R. H. Lupton, R. C. Nichol, D. P. Schneider, M. A. Strauss, M. Tegmark, and D. G. York, The clustering of luminous red galaxies in the Sloan Digital Sky Survey imaging data, Monthly Notices of the Royal Astronomical Society 378 (July, 2007) 852-872, [astro-ph/].

[33] N. Kaiser, Clustering in real space and in redshift space, Monthly Notices of the Royal Astronomical Society 227 (July, 1987) 1-21.

[34] A. J. S. Hamilton, Measuring Omega and the real correlation function from the redshift correlation function, The Astrophysical Journal Letters 385 (Jan., 1992) L5-L8.

[35] T. Matsubara, The Correlation Function in Redshift Space: General Formula with Wide-Angle Effects and Cosmological Distortions, The Astrophysical Journal 535 (May, 2000) 1-23, [astro-ph/9908056].

[36] V. J. Martínez and E. Saar, Statistics of the Galaxy Distribution. Chapman, 2002.

[37] G. B. Arfken and H. J. Weber, Mathematical methods for physicists 6th ed. Elsevier, 2005.

[38] K. B. Fisher, O. Lahav, Y. Hoffman, D. Lynden-Bell, and S. Zaroubi, Wiener reconstruction of density, velocity and potential fields from all-sky galaxy redshift surveys, Monthly Notices of the Royal Astronomical Society 272 (Feb., 1995) 885-908, [astro-ph/9406009]. 
[39] P. G. Castro, A. F. Heavens, and T. D. Kitching, Weak lensing analysis in three dimensions, Physical Review D 72 (July, 2005) 023516, [astro-ph/0503479].

[40] J. Yoo and V. Desjacques, All-sky analysis of the general relativistic galaxy power spectrum, Physical Review D 88 (July, 2013) 023502, [arXiv:1301.4501].

[41] M. Kerscher, The geometry of second-order statistics - biases in common estimators, Astronomy and Astrophysics 343 (Mar., 1999) 333-347, [astro-ph/9811300].

[42] M. Kerscher, I. Szapudi, and A. S. Szalay, A Comparison of Estimators for the Two-Point Correlation Function, The Astrophysical Journal Letters 535 (May, 2000) L13-L16, [astro-ph/9912088].

[43] S. Salazar-Albornoz, A. G. Sánchez, N. D. Padilla, and C. M. Baugh, Clustering tomography: measuring cosmological distances through angular clustering in thin redshift shells, ArXiv e-prints (Feb., 2014) [arXiv:1402.3590].

[44] P. J. E. Peebles, Statistical Analysis of Catalogs of Extragalactic Objects. I. Theory, The Astrophysical Journal 185 (Oct., 1973) 413-440.

[45] P. J. E. Peebles and M. G. Hauser, Statistical Analysis of Catalogs of Extragalactic Objects. III. The Shane-Wirtanen and Zwicky Catalogs, The Astrophysical Journal Supplement Series 28 (Nov., 1974) 19.

[46] A. J. S. Hamilton, Toward Better Ways to Measure the Galaxy Correlation Function, The Astrophysical Journal 417 (Nov., 1993) 19.

[47] M. Davis and P. J. E. Peebles, A survey of galaxy redshifts. V - The two-point position and velocity correlations, The Astrophysical Journal 267 (Apr., 1983) 465-482.

[48] S. D. Landy and A. S. Szalay, Bias and variance of angular correlation functions, The Astrophysical Journal 412 (July, 1993) 64-71.

[49] M.-J. Pons-Bordería, V. J. Martínez, D. Stoyan, H. Stoyan, and E. Saar, Comparing Estimators of the Galaxy Correlation Function, The Astrophysical Journal 523 (Oct., 1999) 480-491, [astro-ph/9906344].

[50] W. Hu and B. Jain, Joint galaxy-lensing observables and the dark energy, Physical Review D 70 (Aug., 2004) 043009, [astro-ph/0312395].

[51] B. Leistedt, H. V. Peiris, D. J. Mortlock, A. Benoit-Lévy, and A. Pontzen, Estimating the large-scale angular power spectrum in the presence of systematics: a case study of Sloan Digital Sky Survey quasars, Monthly Notices of the Royal Astronomical Society 435 (Nov., 2013) 1857-1873, [arXiv:1306.0005].

[52] K. M. Górski, E. Hivon, A. J. Banday, B. D. Wandelt, F. K. Hansen, M. Reinecke, and M. Bartelmann, HEALPix: A Framework for High-Resolution Discretization and Fast Analysis of Data Distributed on the Sphere, The Astrophysical Journal 622 (Apr., 2005) 759-771, [astro-ph/0409513]. 
[53] D. J. C. Mackay, Information Theory, Inference and Learning Algorithms. Cambridge University Press, Oct., 2003.

[54] R. Trotta, Bayes in the sky: Bayesian inference and model selection in cosmology, Contemporary Physics 49 (Mar., 2008) 71-104, [arXiv:0803.4089].

[55] N. Metropolis, A. W. Rosenbluth, M. N. Rosenbluth, A. H. Teller, and E. Teller, Equation of State Calculations by Fast Computing Machines, Journal of Chemical Physics 21 (June, 1953) 1087-1092.

[56] S. Salinas, Introduçao à física estatística. Edusp, São Paulo, 2013.

[57] D. Foreman-Mackey, D. W. Hogg, D. Lang, and J. Goodman, emcee: The MCMC Hammer, The Publications of the Astronomical Society of the Pacific 125 (Mar., 2013) 306-312, [arXiv:1202.3665].

[58] D. Parkinson and A. R. Liddle, Bayesian Model Averaging in Astrophysics: A Review, ArXiv e-prints (Feb., 2013) [arXiv:1302.1721].

[59] J. Goodman and J. Weare, Ensemble samplers with affine invariance, Communications in Applied Mathematics and Computational Science 5 (2010).

[60] J. Christen, "A general purpose scale-independent MCMC algorithm." technical report I-07-16, CIMAT, Guanajuato, 2007.

[61] U. Seljak and M. Zaldarriaga, A line of sight approach to cosmic microwave background anisotropies, Astrophys. J. 469 (1996) 437-444, [astro-ph/9603033].

[62] M. Zaldarriaga, U. Seljak, and E. Bertschinger, Integral solution for the microwave background anisotropies in nonflat universes, Astrophys.J. 494 (1998) 491-502, [astro-ph/9704265].

[63] M. Doran, CMBEASY: an object oriented code for the cosmic microwave background, Journal of Cosmology and Astroparticle Physics 10 (Oct., 2005) 11, [astro-ph/0302138].

[64] J. Lesgourgues, The Cosmic Linear Anisotropy Solving System (CLASS) I: Overview, ArXiv e-prints (Apr., 2011) [arXiv:1104.2932].

[65] D. Blas, J. Lesgourgues, and T. Tram, The Cosmic Linear Anisotropy Solving System (CLASS). Part II: Approximation schemes, Journal of Cosmology and Astroparticle Physics 7 (July, 2011) 34, [arXiv:1104.2933].

[66] M. Colless, B. A. Peterson, C. Jackson, J. A. Peacock, S. Cole, P. Norberg, I. K. Baldry, C. M. Baugh, J. Bland-Hawthorn, T. Bridges, R. Cannon, C. Collins, W. Couch, N. Cross, G. Dalton, R. De Propris, S. P. Driver, G. Efstathiou, R. S. Ellis, C. S. Frenk, K. Glazebrook, O. Lahav, I. Lewis, S. Lumsden, S. Maddox, D. Madgwick, W. Sutherland, and K. Taylor, The 2dF Galaxy Redshift Survey: Final Data Release, ArXiv Astrophysics e-prints (June, 2003) [astro-ph/0306581].

[67] M. J. Drinkwater, R. J. Jurek, C. Blake, D. Woods, K. A. Pimbblet, K. Glazebrook, R. Sharp, M. B. Pracy, S. Brough, M. Colless, W. J. Couch, S. M. Croom, T. M. Davis, D. Forbes, K. Forster, D. G. Gilbank, M. Gladders, 
B. Jelliffe, N. Jones, I.-H. Li, B. Madore, D. C. Martin, G. B. Poole, T. Small, E. Wisnioski, T. Wyder, and H. K. C. Yee, The WiggleZ Dark Energy Survey: survey design and first data release, Monthly Notices of the Royal Astronomical Society 401 (Jan., 2010) 1429-1452, [arXiv:0911.4246].

[68] D. G. York, J. Adelman, J. E. Anderson, Jr., S. F. Anderson, J. Annis, N. A. Bahcall, J. A. Bakken, R. Barkhouser, S. Bastian, E. Berman, W. N. Boroski, S. Bracker, C. Briegel, J. W. Briggs, J. Brinkmann, R. Brunner, S. Burles, L. Carey, M. A. Carr, F. J. Castander, B. Chen, P. L. Colestock, A. J. Connolly, J. H. Crocker, I. Csabai, P. C. Czarapata, J. E. Davis, M. Doi, T. Dombeck, D. Eisenstein, N. Ellman, B. R. Elms, M. L. Evans, X. Fan, G. R. Federwitz, L. Fiscelli, S. Friedman, J. A. Frieman, M. Fukugita, B. Gillespie, J. E. Gunn, V. K. Gurbani, E. de Haas, M. Haldeman, F. H. Harris, J. Hayes, T. M. Heckman, G. S. Hennessy, R. B. Hindsley, S. Holm, D. J. Holmgren, C.-h. Huang, C. Hull, D. Husby, S.-I. Ichikawa, T. Ichikawa, Ž. Ivezić, S. Kent, R. S. J. Kim, E. Kinney, M. Klaene, A. N. Kleinman, S. Kleinman, G. R. Knapp, J. Korienek, R. G. Kron, P. Z. Kunszt, D. Q. Lamb, B. Lee, R. F. Leger, S. Limmongkol, C. Lindenmeyer, D. C. Long, C. Loomis, J. Loveday, R. Lucinio, R. H. Lupton, B. MacKinnon, E. J. Mannery, P. M. Mantsch, B. Margon, P. McGehee, T. A. McKay,

A. Meiksin, A. Merelli, D. G. Monet, J. A. Munn, V. K. Narayanan, T. Nash, E. Neilsen, R. Neswold, H. J. Newberg, R. C. Nichol, T. Nicinski, M. Nonino, N. Okada, S. Okamura, J. P. Ostriker, R. Owen, A. G. Pauls, J. Peoples, R. L. Peterson, D. Petravick, J. R. Pier, A. Pope, R. Pordes, A. Prosapio,

R. Rechenmacher, T. R. Quinn, G. T. Richards, M. W. Richmond, C. H. Rivetta, C. M. Rockosi, K. Ruthmansdorfer, D. Sandford, D. J. Schlegel, D. P. Schneider, M. Sekiguchi, G. Sergey, K. Shimasaku, W. A. Siegmund, S. Smee, J. A. Smith, S. Snedden, R. Stone, C. Stoughton, M. A. Strauss, C. Stubbs, M. SubbaRao, A. S. Szalay, I. Szapudi, G. P. Szokoly, A. R. Thakar, C. Tremonti, D. L. Tucker, A. Uomoto, D. Vanden Berk, M. S. Vogeley, P. Waddell, S.-i. Wang, M. Watanabe, D. H. Weinberg, B. Yanny, N. Yasuda, and SDSS Collaboration, The Sloan Digital Sky Survey: Technical Summary, The Astronomical Journal 120 (Sept., 2000) 1579-1587, [astro-ph/0006396].

[69] A. J. Ross, S. Ho, A. J. Cuesta, R. Tojeiro, W. J. Percival, D. Wake, K. L. Masters, R. C. Nichol, A. D. Myers, F. de Simoni, H. J. Seo,

C. Hernández-Monteagudo, R. Crittenden, M. Blanton, J. Brinkmann, L. A. N. da Costa, H. Guo, E. Kazin, M. A. G. Maia, C. Maraston, N. Padmanabhan, F. Prada, B. Ramos, A. Sanchez, E. F. Schlafly, D. J. Schlegel, D. P. Schneider, R. Skibba, D. Thomas, B. A. Weaver, M. White, and I. Zehavi, Ameliorating systematic uncertainties in the angular clustering of galaxies: a study using the SDSS-III, Monthly Notices of the Royal Astronomical Society 417 (Oct., 2011) 1350-1373, [arXiv:1105.2320].

[70] C. Blake and S. Bridle, Cosmology with photometric redshift surveys, Monthly Notices of the Royal Astronomical Society 363 (Nov., 2005) 1329-1348, [astro-ph/0411713].

[71] T. Budavári, A. J. Connolly, A. S. Szalay, I. Szapudi, I. Csabai, R. Scranton, N. A. Bahcall, J. Brinkmann, D. J. Eisenstein, J. A. Frieman, M. Fukugita, J. E. Gunn, D. Johnston, S. Kent, J. N. Loveday, R. H. Lupton, M. Tegmark, A. R. 
Thakar, B. Yanny, D. G. York, and I. Zehavi, Angular Clustering with Photometric Redshifts in the Sloan Digital Sky Survey: Bimodality in the Clustering Properties of Galaxies, The Astrophysical Journal 595 (Sept., 2003) 59-70, [astro-ph/0305603].

[72] E. Komatsu, K. M. Smith, J. Dunkley, C. L. Bennett, B. Gold, G. Hinshaw, N. Jarosik, D. Larson, M. R. Nolta, L. Page, D. N. Spergel, M. Halpern, R. S. Hill, A. Kogut, M. Limon, S. S. Meyer, N. Odegard, G. S. Tucker, J. L. Weiland, E. Wollack, and E. L. Wright, Seven-year Wilkinson Microwave Anisotropy Probe (WMAP) Observations: Cosmological Interpretation, The Astrophysical Journal Supplement Series 192 (Feb., 2011) 18, [arXiv:1001.4538].

[73] W. H. Press, S. A. Teukolsky, W. T. Vetterling, and B. P. Flannery, Numerical recipes in $\mathrm{C}++$ : the art of scientific computing. Cambridge University Press, New York, NY, USA, 3 ed., 2007.

[74] A. J. S. Hamilton, Uncorrelated modes of the non-linear power spectrum, Monthly Notices of the Royal Astronomical Society 312 (Feb., 2000) 257-284, [astro-ph/9905191].

[75] M. Frigo and S. G. Johnson, The design and implementation of FFTW3, Proceedings of the IEEE 93 (2005), no. 2 216-231. Special issue on "Program Generation, Optimization, and Platform Adaptation".

[76] F. Simpson, J. A. Peacock, and P. Simon, Locating the baryon acoustic peak, Physical Review D 79 (Mar., 2009) 063508, [arXiv:0901.3085].

[77] E. Sánchez, A. Carnero, J. García-Bellido, E. Gaztañaga, F. de Simoni, M. Crocce, A. Cabré, P. Fosalba, and D. Alonso, Tracing the sound horizon scale with photometric redshift surveys, Monthly Notices of the Royal Astronomical Society 411 (Feb., 2011) 277-288, [arXiv:1006.3226].

[78] D. P. Finkbeiner, N. Padmanabhan, D. J. Schlegel, M. A. Carr, J. E. Gunn, C. M. Rockosi, M. Sekiguchi, R. H. Lupton, G. R. Knapp, Ž. Ivezić, M. R. Blanton, D. W. Hogg, J. K. Adelman-McCarthy, J. Annis, J. Hayes, E. Kinney, D. C. Long, U. Seljak, M. A. Strauss, B. Yanny, M. A. Agüeros, S. S. Allam, S. F. Anderson, N. A. Bahcall, I. K. Baldry, M. Bernardi, W. N. Boroski, J. W. Briggs, J. Brinkmann, R. J. Brunner, T. Budavári, F. J. Castander, K. R. Covey, I. Csabai, M. Doi, F. Dong, D. J. Eisenstein, X. Fan, S. D. Friedman, M. Fukugita, B. Gillespie, E. K. Grebel, V. K. Gurbani, E. de Haas, F. H. Harris, J. S. Hendry, G. S. Hennessy, S. Jester, D. E. Johnston, A. M. Jorgensen, M. Jurić, S. M. Kent, A. Y. Kniazev, J. Krzesiński, R. F. Leger, H. Lin, J. Loveday, E. Mannery, D. Martínez-Delgado, P. M. McGehee, A. Meiksin, J. A. Munn, E. H. Neilsen, Jr., P. R. Newman, A. Nitta, G. Pauls, T. R. Quinn, R. R. Rafikov, G. T. Richards, M. W. Richmond, D. P. Schneider, J. Schroeder, K. Shimasaku, W. A. Siegmund, J. A. Smith, S. A. Snedden, A. Stebbins, A. S. Szalay, G. P. Szokoly, M. Tegmark, D. L. Tucker, A. Uomoto, D. E. Vanden Berk, D. H. Weinberg, A. A. West, N. Yasuda, D. R. Yocum, D. G. York, and I. Zehavi, Sloan Digital Sky Survey Imaging of Low Galactic Latitude Fields: Technical Summary and Data Release, The Astronomical Journal 128 (Nov., 2004) 2577-2592, [astro-ph/0409700]. 
[79] A. Collister, O. Lahav, C. Blake, R. Cannon, S. Croom, M. Drinkwater, A. Edge, D. Eisenstein, J. Loveday, R. Nichol, K. Pimbblet, R. de Propris, I. Roseboom, N. Ross, D. P. Schneider, T. Shanks, and D. Wake, MegaZ-LRG: a photometric redshift catalogue of one million SDSS luminous red galaxies, Monthly Notices of the Royal Astronomical Society 375 (Feb., 2007) 68-76, [astro-ph/0607630].

[80] K. N. Abazajian, J. K. Adelman-McCarthy, M. A. Agüeros, S. S. Allam, C. Allende Prieto, D. An, K. S. J. Anderson, S. F. Anderson, J. Annis, N. A. Bahcall, and et al., The Seventh Data Release of the Sloan Digital Sky Survey, The Astrophysical Journal Supplement Series 182 (June, 2009) 543-558, [arXiv:0812.0649].

[81] C. Blake, A. Collister, S. Bridle, and O. Lahav, Cosmological baryonic and matter densities from 600000 SDSS luminous red galaxies with photometric redshifts, Monthly Notices of the Royal Astronomical Society 374 (Feb., 2007) 1527-1548, [astro-ph/0605303].

[82] U. Sawangwit, T. Shanks, F. B. Abdalla, R. D. Cannon, S. M. Croom, A. C. Edge, N. P. Ross, and D. A. Wake, Angular correlation function of 1.5 million luminous red galaxies: clustering evolution and a search for baryon acoustic oscillations, Monthly Notices of the Royal Astronomical Society 416 (Oct., 2011) 3033-3056, [arXiv:0912.0511].

[83] S. A. Thomas, F. B. Abdalla, and O. Lahav, The Angular Power Spectra of Photometric SDSS LRGs, ArXiv e-prints (Nov., 2010) [arXiv:1011.2448].

[84] A. Carnero, E. Sánchez, M. Crocce, A. Cabré, and E. Gaztañaga, Clustering of photometric luminous red galaxies - II. Cosmological implications from the baryon acoustic scale, Monthly Notices of the Royal Astronomical Society 419 (Jan., 2012) 1689-1694, [arXiv:1104.5426].

[85] S. A. Thomas, F. B. Abdalla, and O. Lahav, Excess Clustering on Large Scales in the MegaZ DR7 Photometric Redshift Survey, Physical Review Letters 106 (June, 2011) 241301, [arXiv:1012.2272].

[86] S. Ho, A. Cuesta, H.-J. Seo, R. de Putter, A. J. Ross, M. White, N. Padmanabhan, S. Saito, D. J. Schlegel, E. Schlafly, U. Seljak,

C. Hernández-Monteagudo, A. G. Sánchez, W. J. Percival, M. Blanton, R. Skibba, D. Schneider, B. Reid, O. Mena, M. Viel, D. J. Eisenstein, F. Prada, B. A.

Weaver, N. Bahcall, D. Bizyaev, H. Brewinton, J. Brinkman, L. Nicolaci da Costa, J. R. Gott, E. Malanushenko, V. Malanushenko, B. Nichol, D. Oravetz, K. Pan, N. Palanque-Delabrouille, N. P. Ross, A. Simmons, F. de Simoni, S. Snedden, and C. Yeche, Clustering of Sloan Digital Sky Survey III Photometric Luminous Galaxies: The Measurement, Systematics, and Cosmological Implications, The Astrophysical Journal 761 (Dec., 2012) 14, [arXiv:1201.2137].

[87] H.-J. Seo, S. Ho, M. White, A. J. Cuesta, A. J. Ross, S. Saito, B. Reid, N. Padmanabhan, W. J. Percival, R. de Putter, D. J. Schlegel, D. J. Eisenstein, X. Xu, D. P. Schneider, R. Skibba, L. Verde, R. C. Nichol, D. Bizyaev, H. Brewington, J. Brinkmann, L. A. Nicolaci da Costa, J. R. Gott, III, E. Malanushenko, V. Malanushenko, D. Oravetz, N. Palanque-Delabrouille, 
K. Pan, F. Prada, N. P. Ross, A. Simmons, F. de Simoni, A. Shelden, S. Snedden, and I. Zehavi, Acoustic Scale from the Angular Power Spectra of SDSS-III DR8 Photometric Luminous Galaxies, The Astrophysical Journal 761 (Dec., 2012) 13, [arXiv:1201.2172].

[88] H. Aihara, C. Allende Prieto, D. An, S. F. Anderson, É. Aubourg, E. Balbinot, T. C. Beers, A. A. Berlind, S. J. Bickerton, D. Bizyaev, M. R. Blanton, J. J. Bochanski, A. S. Bolton, J. Bovy, W. N. Brandt, J. Brinkmann, P. J. Brown, J. R. Brownstein, N. G. Busca, H. Campbell, M. A. Carr, Y. Chen, C. Chiappini, J. Comparat, N. Connolly, M. Cortes, R. A. C. Croft, A. J. Cuesta, L. N. da Costa, J. R. A. Davenport, K. Dawson, S. Dhital, A. Ealet, G. L. Ebelke, E. M. Edmondson, D. J. Eisenstein, S. Escoffier, M. Esposito, M. L. Evans, X. Fan, B. Femenía Castellá, A. Font-Ribera, P. M. Frinchaboy, J. Ge, B. A. Gillespie, G. Gilmore, J. I. González Hernández, J. R. Gott, A. Gould, E. K. Grebel, J. E. Gunn, J.-C. Hamilton, P. Harding, D. W. Harris, S. L. Hawley, F. R. Hearty, S. Ho, D. W. Hogg, J. A. Holtzman, K. Honscheid, N. Inada, I. I. Ivans, L. Jiang, J. A. Johnson, C. Jordan, W. P. Jordan, E. A. Kazin, D. Kirkby, M. A. Klaene, G. R. Knapp, J.-P. Kneib, C. S. Kochanek, L. Koesterke, J. A. Kollmeier, R. G. Kron, H. Lampeitl, D. Lang, J.-M. Le Goff, Y. S. Lee, Y.-T. Lin, D. C. Long, C. P. Loomis, S. Lucatello, B. Lundgren, R. H. Lupton, Z. Ma, N. MacDonald, S. Mahadevan, M. A. G. Maia, M. Makler, E. Malanushenko, V. Malanushenko, R. Mandelbaum, C. Maraston, D. Margala, K. L. Masters, C. K. McBride, P. M. McGehee, I. D. McGreer, B. Ménard, J. Miralda-Escudé, H. L. Morrison, F. Mullally, D. Muna, J. A. Munn, H. Murayama, A. D. Myers, T. Naugle, A. F. Neto, D. C. Nguyen, R. C. Nichol, R. W. O’Connell, R. L. C. Ogando, M. D. Olmstead, D. J. Oravetz, N. Padmanabhan, N. Palanque-Delabrouille, K. Pan, P. Pandey, I. Pâris, W. J. Percival, P. Petitjean, R. Pfaffenberger, J. Pforr, S. Phleps, C. Pichon, M. M. Pieri, F. Prada, A. M. Price-Whelan, M. J. Raddick, B. H. F. Ramos, C. Reylé, J. Rich, G. T. Richards, H.-W. Rix, A. C. Robin, H. J. Rocha-Pinto, C. M. Rockosi, N. A. Roe, E. Rollinde, A. J. Ross, N. P. Ross, B. M. Rossetto, A. G. Sánchez, C. Sayres, D. J. Schlegel, K. J. Schlesinger, S. J. Schmidt, D. P. Schneider, E. Sheldon, Y. Shu, J. Simmerer, A. E. Simmons, T. Sivarani, S. A. Snedden, J. S. Sobeck, M. Steinmetz, M. A. Strauss, A. S. Szalay, M. Tanaka, A. R. Thakar, D. Thomas, J. L. Tinker, B. M. Tofflemire, R. Tojeiro, C. A. Tremonti, J. Vandenberg, M. Vargas Magaña, L. Verde, N. P. Vogt, D. A. Wake, J. Wang, B. A. Weaver, D. H. Weinberg, M. White, S. D. M. White, B. Yanny, N. Yasuda, C. Yeche, and I. Zehavi, The Eighth Data Release of the Sloan Digital Sky Survey: First Data from SDSS-III, The Astrophysical Journal Supplement Series 193 (Apr., 2011) 29, [arXiv:1101.1559].

[89] D. J. Eisenstein, D. H. Weinberg, E. Agol, H. Aihara, C. Allende Prieto, S. F. Anderson, J. A. Arns, É. Aubourg, S. Bailey, E. Balbinot, and et al., SDSS-III: Massive Spectroscopic Surveys of the Distant Universe, the Milky Way, and Extra-Solar Planetary Systems, The Astronomical Journal 142 (Sept., 2011) 72, [arXiv: 1101.1529].

[90] L. Anderson, E. Aubourg, S. Bailey, D. Bizyaev, M. Blanton, A. S. Bolton, J. Brinkmann, J. R. Brownstein, A. Burden, A. J. Cuesta, L. A. N. da Costa, K. S. Dawson, R. de Putter, D. J. Eisenstein, J. E. Gunn, H. Guo, J.-C. Hamilton, 
P. Harding, S. Ho, K. Honscheid, E. Kazin, D. Kirkby, J.-P. Kneib, A. Labatie, C. Loomis, R. H. Lupton, E. Malanushenko, V. Malanushenko, R. Mandelbaum, M. Manera, C. Maraston, C. K. McBride, K. T. Mehta, O. Mena, F. Montesano, D. Muna, R. C. Nichol, S. E. Nuza, M. D. Olmstead, D. Oravetz, N. Padmanabhan, N. Palanque-Delabrouille, K. Pan, J. Parejko, I. Pâris, W. J. Percival, P. Petitjean, F. Prada, B. Reid, N. A. Roe, A. J. Ross, N. P. Ross, L. Samushia, A. G. Sánchez, D. J. Schlegel, D. P. Schneider, C. G. Scóccola, H.-J. Seo, E. S. Sheldon, A. Simmons, R. A. Skibba, M. A. Strauss, M. E. C. Swanson, D. Thomas, J. L. Tinker, R. Tojeiro, M. V. Magaña, L. Verde, C. Wagner, D. A. Wake, B. A. Weaver, D. H. Weinberg, M. White, X. Xu, C. Yèche, I. Zehavi, and G.-B. Zhao, The clustering of galaxies in the SDSS-III Baryon Oscillation Spectroscopic Survey: baryon acoustic oscillations in the Data Release 9 spectroscopic galaxy sample, Monthly Notices of the Royal Astronomical Society 427 (Dec., 2012) 3435-3467, [arXiv:1203.6594].

[91] A. A. Collister and O. Lahav, ANNz: Estimating Photometric Redshifts Using Artificial Neural Networks, The Publications of the Astronomical Society of the Pacific 116 (Apr., 2004) 345-351, [astro-ph/0311058].

[92] M. Crocce, E. Gaztañaga, A. Cabré, A. Carnero, and E. Sánchez, Clustering of photometric luminous red galaxies - I. Growth of structure and baryon acoustic feature, Monthly Notices of the Royal Astronomical Society 417 (Nov., 2011) 2577-2591, [arXiv:1104.5236].

[93] A. Lewis and S. Bridle, Cosmological parameters from CMB and other data: a Monte- Carlo approach, Phys. Rev. D66 (2002) 103511, [astro-ph/0205436].

[94] O. Ilbert, S. Arnouts, H. J. McCracken, M. Bolzonella, E. Bertin, O. Le Fèvre, Y. Mellier, G. Zamorani, R. Pellò, A. Iovino, L. Tresse, V. Le Brun, D. Bottini, B. Garilli, D. Maccagni, J. P. Picat, R. Scaramella, M. Scodeggio, G. Vettolani, A. Zanichelli, C. Adami, S. Bardelli, A. Cappi, S. Charlot, P. Ciliegi, T. Contini, O. Cucciati, S. Foucaud, P. Franzetti, I. Gavignaud, L. Guzzo, B. Marano, C. Marinoni, A. Mazure, B. Meneux, R. Merighi, S. Paltani, A. Pollo, L. Pozzetti, M. Radovich, E. Zucca, M. Bondi, A. Bongiorno, G. Busarello, S. de La Torre, L. Gregorini, F. Lamareille, G. Mathez, P. Merluzzi, V. Ripepi, D. Rizzo, and D. Vergani, Accurate photometric redshifts for the CFHT legacy survey calibrated using the VIMOS VLT deep survey, Astronomy and Astrophysics 457 (Oct., 2006) 841-856, [astro-ph/0603217].

[95] R. F. Quadri and R. J. Williams, Quantifying Photometric Redshift Errors in the Absence of Spectroscopic Redshifts, The Astrophysical Journal 725 (Dec., 2010) 794-802, [arXiv:0910.2704].

[96] D. Alonso, CUTE solutions for two-point correlation functions from large cosmological datasets, ArXiv e-prints (Oct., 2012) [arXiv:1210.1833].

[97] L. Anderson, E. Aubourg, S. Bailey, F. Beutler, A. S. Bolton, J. Brinkmann, J. R. Brownstein, C.-H. Chuang, A. J. Cuesta, K. S. Dawson, D. J. Eisenstein, S. Ho, K. Honscheid, E. A. Kazin, D. Kirkby, M. Manera, C. K. McBride, O. Mena, R. C. Nichol, M. D. Olmstead, N. Padmanabhan, N. Palanque-Delabrouille, W. J. Percival, F. Prada, A. J. Ross, N. P. Ross, A. G. Sánchez, L. Samushia, D. J. 
Schlegel, D. P. Schneider, H.-J. Seo, M. A. Strauss, D. Thomas, J. L. Tinker, R. Tojeiro, L. Verde, D. Wake, D. H. Weinberg, X. Xu, and C. Yeche, The clustering of galaxies in the SDSS-III Baryon Oscillation Spectroscopic Survey: measuring $D_{A}$ and $H$ at $z=0.57$ from the baryon acoustic peak in the Data Release 9 spectroscopic Galaxy sample, Monthly Notices of the Royal Astronomical Society (Jan., 2014) [arXiv:1303.4666].

[98] M. Nakahara, Geometry, Topology and Physics, Second Edition. Graduate student series in physics. Taylor \& Francis, 2003.

[99] J. M. Stewart and M. Walker, Perturbations of space-times in general relativity, Royal Society of London Proceedings Series A 341 (Oct., 1974) 49-74.

[100] J. C. A. Barata, "Curso de Física-Matemática." http://denebola.if.usp.br/ jbarata/Notas_de_aula/capitulos.html, 2013.

[101] E. R. Harrison, Normal Modes of Vibrations of the Universe, Reviews of Modern Physics 39 (Oct., 1967) 862-882.

[102] L. F. Abbott and R. K. Schaefer, A general, gauge-invariant analysis of the cosmic microwave anisotropy, The Astrophysical Journal 308 (Sept., 1986) 546-562.

[103] T. Tram, Computation of hyperspherical Bessel functions, ArXiv e-prints (Nov., 2013) [arXiv: 1311.0839].

[104] "NIST Digital Library of Mathematical Functions." http://dlmf.nist.gov/, Release 1.0.6 of 2013-05-06. Online companion to [107].

[105] M. Abramowitz and I. A. Stegun, Handbook of mathematical functions with formulas, graphs, and mathematical tables. Dover Books on Advanced Mathematics, New York: Dover, 1965.

[106] A. J. S. Hamilton, Linear Redshift Distortions: a Review, in The Evolving Universe (D. Hamilton, ed.), vol. 231 of Astrophysics and Space Science Library, p. 185, 1998. astro-ph/9708102.

[107] F. W. J. Olver, D. W. Lozier, R. F. Boisvert, and C. W. Clark, eds., NIST Handbook of Mathematical Functions. Cambridge University Press, New York, NY, 2010. Print companion to [104]. 